\title{
Adriana Karlstroem
}

\section{Estimação de Posição e Quantificação de Erro Utilizando Geometria Epipolar entre Imagens}

Dissertação apresentada à Escola Politécnica da Universidade de São Paulo para obtenção do Título de Mestre em Engenharia Mecânica. 


\title{
Adriana Karlstroem
}

\section{Estimação de Posição e Quantificação de Erro Utilizando Geometria Epipolar entre Imagens}

\author{
Dissertação apresentada à Escola Po- \\ litécnica da Universidade de São Paulo \\ para obtenção do Título de Mestre em \\ Engenharia Mecânica. \\ Área de concentração: \\ Engenharia de Controle e Automação \\ Mecânica \\ Orientador: \\ Prof. Dr. Fabio Kawaoka Takase
}


Ficha Catalográfica

Karlstroem, Adriana

Estimação de Posição e Quantificação de Erro Utilizando Geometria Epipolar entre Imagens. São Paulo, 2007. 156 p.

Dissertação (Mestrado) - Escola Politécnica da Universidade de São Paulo. Departamento de Engenharia Mecânica.

1. Estimação de posição 2. Geometria epipolar 3. Visão Computacional I. Universidade de São Paulo. Escola Politécnica. Departamento de Engenharia Mecânica. II. t. 
a meu pai. 


\section{Agradecimentos}

Agradeço a meu orientador, Prof. Dr. Fabio Kawaoka Takase pela oportunidade de realizar este trabalho, pela paciência, dedicação e confiança. Agradeço a Luis Henrique Maciel da Rosa pela oportunidade de emprego que me permitiu levar em paralelo os estudos de mestrado. Agradeço aos colegas do Laboratório de Sistemas Embarcados por toda ajuda durante minhas pesquisas. Agradeço a meus pais, pelo apoio constante em tudo que fiz. 


\section{Resumo}

A estimação de posição é o resultado direto da reconstrução de cenas, um dos ramos da visão computacional. É também uma informação importante para o controle de sistemas mecatrônicos, e em especial para os sistemas robóticos autônomos. Como uma aplicação de engenharia, o desempenho de tal sistema deve ser avaliado em termos de eficiência e eficácia, medidas traduzidas respectivamente pelo custo de processamento e pela quantificação do erro. A geometria epipolar é um campo da visão computacional que fornece formalismo matemático e técnicas de reconstrução de cenas a partir de uma par de imagens, através de pontos correspondentes entre elas. Através deste formalismo é possível determinar a incerteza dos métodos de estimação de posição, que são relativamente simples e podem atingir boa precisão. Dentre os sistemas robóticos autônomos destacam-se os ROVs - do inglês "Remotely Operated Vehicles" - ou veículos operados remotamente, muito utilizados em tarefas submarinas, e cuja necessidade crescente de autonomia motiva o desenvolvimento de um sensor de visão com características de baixo consumo de energia, flexibilidade e inteligência. Este sensor pode consistir de uma câmera CCD e algoritmos de reconstrução de cena baseados em geometria epipolar entre imagens. Este estudo visa fornecer um comparativo de resultados práticos da estimação de posição através da geometria epipolar entre imagens, como parte da implementação de um sensor de visão para robôs autônomos. Os conceitos teóricos abordados são: geometria projetiva, modelo de câmera, geometria epipolar, matriz fundamental, reconstrução projetiva, re-construção métrica, algoritmos de determinação da matriz fundamental, algoritmos de reconstrução métrica, incerteza da matriz fundamental e complexidade computacional. Os resultados práticos baseiam-se em simulações através de imagens geradas por computador e em montagens experimentais feitas em laboratório que simulam situações práticas. O processo de estimação de posição foi realizado através da implementação em MATLAB ${ }^{\circledR} 6.5$ dos algoritmos apresentados na parte teórica, e os resultados comparados e analisados quanto ao erro e complexidade de execução. Dentre as principais conclusões é apresentado a melhor escolha para a implementação de sensor de visão de propósito geral - o Algoritmo de 8 Pontos Correspondentes Normalizado. São apresentadas também as condições de utilização de cada método e os cuidados necessários na interpretação dos resultados. 


\section{Abstract}

Position estimation is the direct result of scene reconstruction, one of computer vision's fields. It is also an important information for the control of mechanical systems - specially the autonomous robotic systems. As an engineering application, those systems' performance must be evaluated in terms of efficiency and effectiveness, measured by processing costs and error quantification. The epipolar geometry is a field of computer vision that supply mathematical formalism and scene reconstruction techniques that are based on the correspondences between two images. Through this formalism it is possible to stipulate the uncertainty of the position estimation methods that are relatively simple and can give good accuracy. Among the autonomous robotic systems, the ROVs - Remotely Operated Vehicles - are of special interest, mostly employed in submarine activities, and whose crescent autonomy demand motivates the development of a vision sensor of low power consumption, flexibility and intelligence. This sensor may be constructed with a CCD camera and the scene reconstruction algorithms based on epipolar geometry. This work aims to build a comparison of practical results of position estimation through epipolar geometry, as part of a vision sensor implementation for autonomous robots. The theory presented in this work comprises of: projective geometry, camera model, epipolar geometry, fundamental matrix, projective reconstruction, metric reconstruction, fundamental matrix algorithms, metric reconstruction algorithms, fundamental matrix uncertainty, and computational complexity. The practical results are based on computer generated simulations and experimental assemblies that emulate practical issues. The position estimation was carried out by MATLAB ${ }^{\circledR} 6.5$ implementations of the algorithms analyzed in the theoretical part, and the results are compared and analyzed in respect of the error and the execution complexity. The main conclusions are that the best algorithm choice for the implementation of a general purpose vision sensor is the Normalized 8 Point Algorithm, and the usage conditions of each method, besides the special considerations that must be observed at the interpretation of the results. 


\title{
Sumário
}

\section{Lista de Figuras}

\author{
Lista de Tabelas
}

\section{Convenções e Lista de Símbolos}

1 Introdução 1

1.1 Visão Geral . . . . . . . . . . . . . . . . . . . . . . . . 1

1.2 Justificativa . . . . . . . . . . . . . . . . . . 4

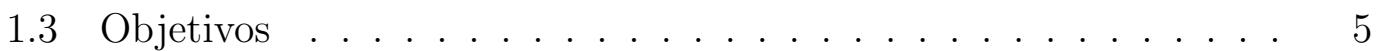

1.4 Organização . . . . . . . . . . . . . . . . . . . . 6

I Conceitos Teóricos $\quad 8$

2 Geometria Projetiva $\quad 9$

2.1 Geometria Projetiva 2D . . . . . . . . . . . . . . . 9

2.2 Geometria Projetiva 3D . . . . . . . . . . . . . . 16

3 Modelo de Câmera $\quad 24$

3.1 Câmeras Finitas . . . . . . . . . . . . . . . . . . . 24

3.1.1 Modelo Básico de Câmera de Orifício . . . . . . . . . . . 25

3.1.2 Projeção Central em Coordenadas Homogêneas .... . 25

3.1.3 Deslocamento do Ponto Principal . . . . . . . . . . . . 26

3.1.4 Rotação e Translação de Câmera . . . . . . . . . . . . . . 27

3.1.5 Câmeras CCD . . . . . . . . . . . . . . . . . . . . 28

3.1 .6 Câmera Projetiva Finita . . . . . . . . . . . . . . . 29 
3.1.7 Câmera Projetiva Geral . . . . . . . . . . . . . . 30

3.2 Anatomia da Câmera Projetiva . . . . . . . . . . . . 30

3.3 Lentes: Distorção Radial . . . . . . . . . . . . . . . . . . . . . 34

3.4 Centro de Câmera . . . . . . . . . . . . . . . 36

3.4.1 Deslocamento do Plano de Imagem . . . . . . . . . . . 37

3.4.2 Rotação de Câmera . . . . . . . . . . . . . . . . 38

4 Geometria Epipolar e Matriz Fundamental 40

4.1 Geometria Epipolar . . . . . . . . . . . . . . . . 40

4.2 Matriz Fundamental ................... . . 41

4.2.1 Dedução Geométrica . . . . . . . . . . . . . . . . . 42

4.2 .2 Dedução Algébrica . . . . . . . . . . . . . . . . . . 43

4.2.3 Condição de Correspondência . . . . . . . . . . . . . . 44

4.2.4 Propriedades da Matriz Fundamental . . . . . . . . . . 45

4.3 Movimentos Especiais de Câmera . . . . . . . . . . . . . 47

4.3.1 Movimento de Translação Pura . . . . . . . . . . . . . 47

4.3.2 Movimento Geral . . . . . . . . . . . . . . . . . . . . . . 48

4.3 .3 Movimento planar puro . . . . . . . . . . . . . . . 49

4.4 Matrizes de Câmera . . . . . . . . . . . . . . . 50

4.4.1 Forma Canônica da Matriz de Câmera . . . . . . . . . 50

4.4.2 Ambigüidade Projetiva . . . . . . . . . . . 51

4.4.3 Câmeras Canônicas da Matriz Fundamental . . . . . . . 51

4.4.4 Ambigüidade Reconstrutiva . . . . . . . . . . . . 52

4.4.5 Teorema da Reconstrução Projetiva . . . . . . . . . . . 55

5 Cálculo da Matriz Fundamental $\quad 56$

5.1 Cálculo Básico . . . . . . . . . . . . . . . . 56

5.2 Algoritmo de 8 Pontos Correspondentes . . . . . . . . . . . . 57

5.3 Algoritmo de 7 Pontos Correspondentes . . . . . . . . . . . . . 58 
5.4 Algoritmo de 8 Pontos Correspondentes Normalizado . . . . . . . 58

5.5 Distância Epipolar e Erro Residual . . . . . . . . . . . . . . 59

5.6 Busca Exaustiva . . . . . . . . . . . . . . . 60

5.7 Solução Linear Iterativa (Ponderada) . . . . . . . . . . . . . . 61

6 Incerteza da Matriz Fundamental $\quad 63$

6.1 Método Estatístico . . . . . . . . . . . . . . 63

6.2 Método Analítico . . . . . . . . . . . . . . . . . . 64

6.2.1 Caso Explícito.................... 64

6.2 .2 Caso Implícito . . . . . . . . . . . . . . . . . . . . 64

$\begin{array}{lll}7 & \text { Reconstrução Métrica } & 68\end{array}$

7.1 Matriz Fundamental . . . . . . . . . . . . . . . . 69

7.2 Câmeras Canônicas . . . . . . . . . . . . . . . . . . . . . 69

7.3 Triangulação . . . . . . . . . . . . . . . . . . . 69

7.4 Câmeras Métricas . . . . . . . . . . . . . . . 71

7.5 Erro de Reconstrução . . . . . . . . . . . . . . . . . . . . . 72

7.6 Algoritmo de Reconstrução . . . . . . . . . . . . . . . . . . 72

8 Complexidade Computacional $\quad 74$

8.1 Algoritmo de 8 Pontos Correspondentes Normalizado . . . . . . 75

8.2 Algoritmo de 7 Pontos Correspondentes . . . . . . . . . . . . 75

8.3 Erro Residual . . . . . . . . . . . . . . . . 76

8.4 Busca Exaustiva . . . . . . . . . . . . . . . 76

8.5 Solução Linear Iterativa . . . . . . . . . . . . . . . 76

8.6 Reconstrução . . . . . . . . . . . . . . . . . . . . 77

$\begin{array}{ll}\text { II Resultados Práticos } & 78\end{array}$

$\begin{array}{llr}9 & \text { Resultados } & 79\end{array}$ 
9.1 Conjuntos de Dados . . . . . . . . . . . . . . . . . . 79

9.1 .1 Simulação . . . . . . . . . . . . . . . . 80

9.1.2 Montagem Experimental .............. 81

9.2 Processamento . . . . . . . . . . . . . . . . 87

9.2.1 Matriz Fundamental . . . . . . . . . . . . . 87

9.2.2 Reconstrução Projetiva . . . . . . . . . . . . . . . . 99

9.2.3 Reconstrução Métrica . . . . . . . . . . . . . . . . . . . 99

9.3 Análise . . . . . . . . . . . . . . . . . . . . . 110

9.3.1 Matriz Fundamental . . . . . . . . . . . . . 111

9.3.2 Matrizes de Projeção Canônicas . . . . . . . . . . . . . . . 114

9.3.3 Matrizes de Projeção Métricas . . . . . . . . . . . . . . 115

$\begin{array}{lr}\text { Conclusão } & 118\end{array}$

$\begin{array}{lr}\text { Referências } & 122\end{array}$

$\begin{array}{lr}\text { Apêndice A - Conceitos Adicionais } & 126\end{array}$

A.1 Rotação Conjugada . . . . . . . . . . . . . . . . . . . 126

A.2 Produto Vetorial . . . . . . . . . . . . . . . . 126

A.2.1 Representação Matricial . . . . . . . . . . . . 126

A.2.2 Operação Matricial . . . . . . . . . . . . . . . 127

A.3 Rotação e Translação Tridimensional . . . . . . . . . . . . . . . 127

A.4 Decomposição em Valores Singulares . . . . . . . . . . . . . 128

A.5 Norma de Frobenius . . . . . . . . . . . . . . . . . . . 129

A.6 Normalização . . . . . . . . . . . . . . . . . . . . . . . . . . . . 129

A.7 Valor RMS . . . . . . . . . . . . . . . . . . . . . . . 131

A.8 Algoritmo DLT . . . . . . . . . . . . . . . . . . . . . . . . . . 131

A.9 Complexidade Computacional . . . . . . . . . . . . . . . . . . 132

Apêndice B - Listagens dos Programas $\quad 134$ 
B.1 Cálculo da Matriz Fundamental . . . . . . . . . . . . . . . . . 134

B.1.1 Algoritmo de 7 Pontos Correspondentes . . . . . . . . . 134

B.1.2 Algoritmo de 8 Pontos Correspondentes Normalizado . . . 135

B.1.3 Algoritmo de Busca Exaustiva . . . . . . . . . . . . . . 136

B.1.4 Algoritmo Linear Iterativo . . . . . . . . . . . . . 137

B.2 Matrizes de Projeção Canônicas . . . . . . . . . . . . . . . . . 138

B.3 Triangulação . . . . . . . . . . . . . . . . . . . . . . . . 138

B.4 Matrizes de Projeção Métricas . . . . . . . . . . . . . . . 139

B.5 Erros . . . . . . . . . . . . . . . . 139

B.5.1 Erro Residual . . . . . . . . . . . . . . . . . . . . 139

B.5.2 Erro de Reconstrução . . . . . . . . . . . . . . . . . . . . . 140

B.6 Interface Gráfica . . . . . . . . . . . . . . . . . . . . . . 140

B.7 Outros Programas Acessórios _ . . . . . . . . . . . . . . . 153

B.7.1 Matriz do Produto Vetorial . . . . . . . . . . . . 153

B.7.2 Epipolos . . . . . . . . . . . . . . . . . . 154

B.7.3 Construção dos Dados de Simulação . . . . . . . . . . . . . 154

B.7.4 Visualização . . . . . . . . . . . . . . . . . . . 155 


\section{Lista de Figuras}

2.1 Modelo do plano projetivo. . . . . . . . . . . . . . . . 11

2.2 Exemplo de (a) cônica e (b) cônica dual. . . . . . . . . . . . . . . 12

2.3 Distorção causada pela transformação afim planar. (a) Rotação. (b) Deformação. . . . . . . . . . . . . . . . . . . . . . . . . 15

3.1 Geometria da Câmera de Orifício. . . . . . . . . . . . . 25

3.2 Coordenadas de imagem $(x, y)$ e de câmera $\left(x_{\text {cam }}, y_{\text {cam }}\right) \ldots \ldots 26$

3.3 Transformação Euclidiana entre os sistemas de coordenadas global e de câmera. . . . . . . . . . . . . . . . . . . . . 27

3.4 Vetores coluna da matriz de câmera. . . . . . . . . . . . . . . . . 31

3.5 Planos $\mathbf{p}^{2^{\mathrm{T}}}$ e $\mathbf{p}^{3^{\mathrm{T}}}$ definidos pelas linhas da matriz de câmera. . . . 32

3.6 Profundidade do ponto X. . . . . . . . . . . . . . . . . . . 34

3.7 Distorção Radial $\times$ Distância Focal. (a) Distância focal curta. (b) Distância focal longa. . . . . . . . . . . . . . . . . . . . . 35

3.8 Correção da distorção radial. . . . . . . . . . . . . . . . . . . . . . 35

3.9 Cone de raios com vértice no centro de câmera. . . . . . . . . . . 37

3.10 Exemplo de rotação e translação de câmera. (a) Posição inicial. (b) Posição após rotação. (c) Posição após rotação e translação.

4.1 Geometria de Pontos Correspondentes. (a) Plano Epipolar. (b) Reta Epipolar. . . . . . . . . . . . . . . . . . . . . . . . . 41

4.2 Geometria Epipolar. (a) Relação entre plano epipolar, reta base, epipolos e retas epipolares. (b) Feixe de planos epipolares. . . . . 42

4.3 Homografia 2D entre pontos correspondentes. . . . . . . . . . . 43

4.4 Homografia da Reta Epipolar. (a) Feixes de retas epipolares. (b) Transformação perspectiva entre retas epipolares. . . . . . . . . . 46

4.5 Epipolos do movimento de translação pura da câmera. . . . . . . 47 
4.6 Movimento Geral de Câmera. . . . . . . . . . . . . . . . . . . . . 49

4.7 Ambigüidade Reconstrutiva. (a) Transformação similar. (b) Transformação projetiva. . . . . . . . . . . . . . . . 54

7.1 Triangulação. . . . . . . . . . . . . . . . . 70

9.1 Conjuntos de dados utilizados na estimação de posição. . . . . . . 80

9.2 Imagens do conjunto de dados de simulação. (a) Movimento de translação pura. (b) Movimento planar. . . . . . . . . . . . . . . 81

9.3 O robô KUKA no ambiente do laboratório. . . . . . . . . . . . . . 82

9.4 Sistemas de coordenadas do robô KUKA, conforme o manual. (a) Sistema de coordenadas na flange. (b) Sistema de coordenadas na base. . . . . . . . . . . . . . . . . . 82

9.5 Imagens da primeira montagem experimental. (a) Movimento de translação pura. (b) Movimento geral. . . . . . . . . . . . . . 83

9.6 Segunda montagem experimental. . . . . . . . . . . . . 84

9.7 Imagens da segunda montagem experimental. . . . . . . . . . . 85

9.8 Terceira montagem experimental. . . . . . . . . . . . . 86

9.9 Imagens da terceira montagem experimental. . . . . . . . . . . 86

9.10 Resultados de simulação para movimento de translação pura. (a) Algoritmo de 7 Pontos Correspondentes. (b) Algoritmo de Busca Exaustiva. (c) Algoritmo de 8 Pontos Correspondentes Normalizado. (d) Algoritmo Linear Iterativo. . . . . . . . . . . . . . . 90

9.11 Resultados de simulação no caso de movimento planar. (a) Algoritmo de 7 Pontos Correspondentes. (b) Algoritmo de Busca Exaustiva. (c) Algoritmo de 8 Pontos Correspondentes Normalizado. (d) Algoritmo Linear Iterativo. . . . . . . . . . . . . . . . . 91

9.12 Resultados da primeira montagem experimental no caso de translação pura. (a) Algoritmo de 7 Pontos Correspondentes. (b) Algoritmo de Busca Exaustiva. (c) Algoritmo de 8 Pontos Correspondentes Normalizado. (d) Algoritmo Linear Iterativo. . . . . . . . . . . . . 92 
9.13 Resultados da primeira montagem experimental no caso de movimento geral. (a) Algoritmo de 7 Pontos Correspondentes. (b) Algoritmo de Busca Exaustiva. (c) Algoritmo de 8 Pontos Correspondentes Normalizado. (d) Algoritmo Linear Iterativo. . . . . .

9.14 Resultados do Algoritmo de 7 Pontos Correspondentes na segunda montagem experimental. (a) 01-02. (b) 02-03 (c) 04-05 (d) 01-04. (e) 01-05. (f) 02-04. (g) 02-05.

9.15 Resultados do Algoritmo de Busca Exaustiva na segunda montagem experimental, para os subconjuntos A (esquerda) e B (direita). (a) 01-02. (b) 02-03 (c) 04-05 (d) 01-04. (e) 01-05. (f) 02-04. (g) $02-05 \ldots \ldots \ldots \ldots \ldots \ldots$

9.16 Resultados do Algoritmo de 8 Pontos Correspondentes Normalizado na segunda montagem experimental, para os subconjuntos A (esquerda) e B (direita). (a) 01-02. (b) 02-03 (c) 04-05 (d) 01-04. (e) 01-05. (f) 02-04. (g) 02-05.

9.17 Resultados do Algoritmo Linear Iterativo na segunda montagem experimental, para os subconjuntos A (esquerda) e B (direita). (a) 01-02. (b) 02-03 (c) 04-05 (d) 01-04. (e) 01-05. (f) 02-04. (g) 02-05.

9.18 Resultados do Algoritmo de 7 Pontos Correspondentes na terceira montagem experimental. (a) 00-01. (b) 01-02. (c) 02-03. (d) 03-04. (e) 04-05. (f) 05-06. (g) 12-13. (h) 15-16. (i) 06-07. (j) 13-14. (k) 16-17

9.19 Resultado do Algoritmo de Busca Exaustiva na terceira montagem experimental, para os subconjuntos A (esquerda) e B (direita). (a) 00-01. (b) 01-02. (c) 02-03. (d) 03-04. (e) 04-05. (f) 05-06. (g) 06-07. (h) 12-13. (i) 13-14. (j) 15-16. (k) 16-17. . . . . 101

9.20 Resultado do Algoritmo de 8 Pontos Correspondentes Normalizado na terceira montagem experimental, para os subconjuntos A (esquerda) e B (direita). (a) 00-01. (b) 01-02. (c) 02-03. (d) 03-04. (e) 04-05. (f) 05-06. (g) 06-07. (h) 12-13. (i) 13-14. (j) 15-16. (k) $16-17 \ldots \ldots \ldots \ldots$ 
9.21 Resultado do Algoritmo Linear Iterativo na terceira montagem experimental, para os subconjuntos A (esquerda) e B (direita). (a) 00-01. (b) 01-02. (c) 02-03. (d) 03-04. (e) 04-05. (f) 05-06. (g) 06-07. (h) 12-13. (i) 13-14. (j) 15-16. (k) 16-17. . . . . 103

9.22 Pontos de referência 3D da segunda montagem experimental. (a) 01-02. (b) 02-03 (c) 04-05 (d) 01-04. (e) 01-05. (f) 02-04. (g)

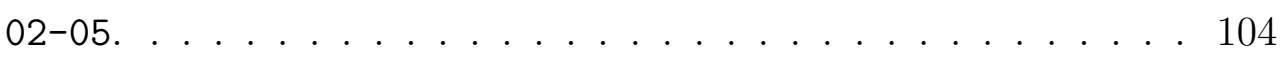

9.23 Pontos de referência 3D da terceira montagem experimental. (a) 00-01. (b) 01-02. (c) 02-03. (d) 03-04. (e) 04-05. (f) 05-06. (g) 06-07. (h) 12-13. (i) 13-14. (j) 15-16. (k) 16-17. . . . . 105

9.24 Pontos de teste 3D da segunda montagem experimental. (a) 01-02. (b) 02-03 (c) 04-05 (d) 01-04. (e) 01-05. (f) 02-04. (g) 02-05. . 107

9.25 Pontos de teste 3D da terceira montagem experimental. (a) 00-01. (b) 01-02. (c) 02-03. (d) 03-04. (e) 04-05. (f) 05-06. (g) 06-07.

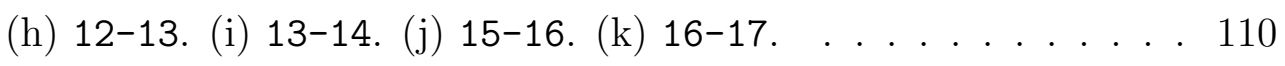
9.26 Resumo da análise dos resultados. . . . . . . . . . . . . . . . . 117 


\section{Lista de Tabelas}

2.1 Propriedades geométricas invariantes das transformações 2D. . . . 21

2.2 Propriedades geométricas invariantes das transformações 3D. . . . 21

8.1 Complexidade computacional de algoritmos de cálculo da matriz fundamental e de reconstrução métrica. . . . . . . . . . . . . . . 74

9.1 Erro residual da simulação $\left(\right.$ pixel $\left.^{2}\right) \ldots \ldots$. . . . . . . . . . 88

9.2 Erro residual da primeira montagem experimental $\left(\right.$ pixel $\left.^{2}\right)$. . . . 89

9.3 Erro residual do Algoritmos de 7 Pontos Correspondentes na segunda montagem experimental $\left(\right.$ pixel $\left.^{2}\right) \ldots \ldots . . \ldots 90$

9.4 Erro residual do Algoritmo de Busca Exaustiva na segunda montagem experimental $\left(\right.$ pixel $\left.^{2}\right) \ldots \ldots . \ldots . \ldots 9 . \ldots 9$

9.5 Erro residual do Algoritmo de 8 Pontos Correspondentes Normalizado na segunda montagem experimental $\left(\right.$ pixel $\left.^{2}\right)$. . . . . . . . 91

9.6 Erro residual do Algoritmo Linear Iterativo na segunda montagem experimental $\left(\right.$ pixel $\left.^{2}\right) \ldots \ldots \ldots$. . . . . . . . . . . . . 92

9.7 Erro residual do Algoritmos de 7 Pontos Correspondentes na terceira montagem experimental $\left(\right.$ pixel $\left.^{2}\right)$. . . . . . . . . . . . . 94

9.8 Erro residual do Algoritmo de Busca Exaustiva na terceira montagem experimental $\left(\right.$ pixel $\left.^{2}\right) \ldots \ldots \ldots$. . . . . . . . . . 96

9.9 Erro residual do Algoritmo de 8 Pontos Correspondentes Normalizado na terceira montagem experimental $\left(\right.$ pixel $\left.^{2}\right) . \quad \ldots . . .97$

9.10 Erro residual do Algoritmo Linear Iterativo na terceira montagem experimental $\left(\right.$ pixel $\left.^{2}\right) \ldots \ldots \ldots \ldots$

9.11 Erro residual dos pontos de referência 3D da segunda montagem

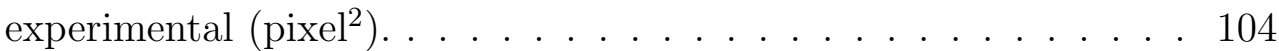

9.12 Erro residual dos pontos de referência 3D na terceira montagem experimental $\left(\right.$ pixel $\left.^{2}\right) \ldots \ldots \ldots \ldots$. . . . . . . . . . . 105 
9.13 Erro de reconstrução métrica dos pontos de referência 3D na segunda montagem experimental $\left(\mathrm{m}^{2}\right)$. . . . . . . . . . 106

9.14 Erro de reconstrução métrica dos pontos de referência 3D na terceira montagem experimental $\left(\mathrm{m}^{2}\right) \ldots \ldots \ldots$. . . . . . . 106

9.15 Erro de reconstrução métrica dos pontos de teste 3D na segunda montagem experimental $\left(\mathrm{m}^{2}\right)$. . . . . . . . . . . . 108

9.16 Erro da reconstrução métrica dos pontos de teste 3D na terceira montagem experimental $\left(\mathrm{m}^{2}\right)$. . . . . . . . . . . . . . 109

9.17 Distância entre os pontos de teste 3D dos blocos K e L na segunda montagem experimental $(\mathrm{m}) \ldots \ldots \ldots 111$

9.18 Distância entre os pontos de teste 3D dos blocos K e L na terceira montagem experimental $(\mathrm{m}) \ldots \ldots \ldots$. . . . . . . . . 112 


\section{Convenções e Lista de Símbolos}

Na notação das fórmulas e no texto, são utilizadas as seguintes convenções:

- letras maiúsculas são matrizes, exemplo: F, P;

- letras maiúsculas em negrito são vetores representando pontos do espaço tridimensional, exemplo: $\mathbf{X}$;

- letras minúsculas em negrito são vetores representando pontos ou retas do espaço bidimensional, exemplo: x, $\mathbf{l}$;

- letras minúsculas em itálico são valores escalares, exemplo: $n, k$;

Os seguintes símbolos são utilizados:

\begin{tabular}{ll}
\hline símbolo & descrição \\
\hline $\mathbf{R}^{\mathbf{2}}$ & espaço vetorial bidimensional. \\
$\mathbf{R}^{\mathbf{3}}$ & espaço vetorial tridimensional. \\
$\mathbf{P}^{\mathbf{2}}$ & espaço projetivo bidimensional. \\
$\mathbf{P}^{\mathbf{3}}$ & espaço projetivo tridimensional. \\
$\mathbf{P}$ & matriz de câmera. \\
$\mathrm{K}$ & matriz de parâmetros intrínsecos de câmera. \\
$\mathbf{R}$ & matriz de rotação de câmera. \\
$\mathbf{t}$ & vetor de translação de câmera. \\
$\mathbf{C}$ & centro de câmera. \\
$\mathbf{F}$ & matriz fundamental. \\
$\mathrm{E}[\mathbf{y}]$ & média do vetor aleatório y. \\
$\boldsymbol{\Lambda}_{\mathbf{y}}$ & covariânça do vetor aleatório $\mathbf{y}$. \\
{$[\bullet]_{\times}$} & representação de produto vetorial. \\
$\|\bullet\|$ & representação de norma. \\
$\mathcal{O}(\bullet)$ & notação Big-Oh de limite assintótico. \\
\hline
\end{tabular}




\section{Introdução}

A estimação de posição é parte de um dos ramos da visão computacional - a reconstrução de cenas. Como tal, é parte importante de aplicações em controle de processos, principalmente no caso de veículos autônomos. Sendo uma aplicação de engenharia, especificamente de sistemas mecânicos, seus resultados devem ser analisados quanto a eficiência e eficácia. Neste caso, a eficiência representa a complexidade de implementação de cada método de estimação de posição, e a eficácia traduz-se na quantificação do erro inerente ao processo, em cada etapa e como um todo. Neste sentido, a geometria epipolar é uma das teorias que fundamentam a visão computacional, e fornece métodos para determinar a reconstrução de cenas a partir de uma par de imagens, das quais a única informação necessária é um conjunto de pontos correspondentes entre elas. Com a informação adicional de alguns pontos de referência, as técnicas derivadas da geometria epipolar permitem a estimação de posição com razoável precisão e moderado custo de processamento.

\section{$1.1 \quad$ Visão Geral}

A visão computacional pode ser definida como a ciência e o conjunto de tecnologias que propiciam a uma máquina a capacidade de percepção visual (MARR, 1982). Como disciplina científica, a visão computacional dedica-se à teoria e às técnicas de construção de sistemas artificiais para obtenção de informação a partir de imagens ou dados multi-dimensionais. Informação é tudo aquilo que possibilita a tomada de decisões. Como a percepção pode ser vista como a extração de informação de sinais sensoriais, a visão computacional pode ser encarada como a investigação científica de sistemas artificiais de percepção a partir de imagens ou dados multi-dimensionais. Como tecnologia, a visão computacional busca a aplicação de suas teorias e modelos no desenvolvimento de sistemas de visão computacional. Tais aplicações incluem a detecção de eventos - como em sistemas de vigilância, organização de informações - como em indexação de seqüências de imagens, a modelagem de objetos e ambientes - como inspeção industrial ou 
modelagem topográfica, e o controle de processos - como um robô industrial ou um veículo autônomo. Assim, os ramos da visão computacional englobam detecção de eventos, tracking, indexação, restauração de imagens, aprendizado, reconhecimento de objetos e reconstrução de cenas.

O campo da visão computacional é diversificado e aberto. Mesmo que os primeiros trabalhos nesta área tenham aparecido na década de 60, somente a partir do final da década de 70 que estudos focados em aplicações práticas começaram a partir do momento em que os computadores passaram a ter capacidade de processamento para trabalhar com grandes conjuntos de dados, como são as imagens. Contudo, estes estudos originaram-se em variadas áreas do conhecimento, de forma que atualmente existe uma miríade de métodos para solucionar casos muito bem definidos de visão computacional (ULLMAN, 1979) - métodos que são específicos para as tarefas que procuram executar e que normalmente não podem ser generalizados para uma gama mais ampla de aplicações. Além disso, muitos destes métodos estão no estágio de pesquisa básica, e na maioria das aplicações práticas da visão computacional, os computadores são préprogramados para resolver um problema particular, onde cada vez mais tem-se utilizado métodos baseados em aprendizado de máquina e inteligência artificial.

Uma parte significativa da inteligência artificial lida com o planejamento e tomada de decisões em sistemas que realizam ações mecânicas como a movimentação de um robô através de uma determinado ambiente. Tipicamente, este processamento requer dados provenientes de um sistema de visão computacional, atuando como um sensor e fornecendo informações de alto nível sobe o ambiente circundante ao robô. Outros elementos da inteligência artificial relacionados à visão computacional são o aprendizado e o reconhecimento de padrões. Assim, a visão computacional muitas vezes é classificada como parte do campo da inteligência artificial ou da ciência da computação (GIBSON, 1955). Outros campos da ciência intimamente relacionados com a visão computacional são a física - no que se refere à óptica e à física de estado-sólido, a neurobiologia - na modelagem de sistemas de visão biológicos, o processamento de sinais - estendendo conceitos de processos estocásticos unidimensionais para o campo bidimensional, e a geometria computacional - na modelagem de sólidos e no estudo de erros de representação numérica (TSUZUKI, 2000), (SHIMADA; TSUZUKI, 2001), (SONG; AN; FOLLECO, 2003).

No caso da reconstrução de cenas e estimação de posição, os estudos baseiamse na matemática - álgebra matricial, estatística e geometria. Com os fundamentos destes campos, desenvolvem-se modelos de câmera, a relação de corres- 
pondência entre imagens e os algoritmos de reconstrução da estrutura espacial (MAYBANK, 1993), (SEMPLE; KNEEBONE, 1952), (TSAI, 1986).

A geometria epipolar é o estudo da correspondência entre imagens (DERICHE et al., 1994), (XU; ZHANG, 1996), (FAUGERAS, 1993). Este estudo traduz a relação entre duas imagens resultantes da projeção de uma cena através de duas câmeras em uma matriz chamada matriz fundamental (LUONG et al., 1993). Esta matriz fundamental transforma pontos de uma imagem em retas da outra imagem, e determina a condição de correspondência entre os pontos de ambas imagens. A partir da matriz fundamental é possível determinar as matrizes de projeção representando as câmeras que produziram as imagens, a menos de uma ambigüidade projetiva. Dados alguns pontos de referência no espaço tridimensional, é possível recuperar a estrutura da cena original apenas conhecendo-se a matriz fundamental (HARTLEY, 1994), (SEMPLE; KNEEBONE, 1952), (HARTLEY; ZISSERMAN, 2000), (TORR; ZISSERMAN, 1997), (HARTLEY, 1997b), (CRIMINISI; REID; ZISSERMAN, 1999), (CRIMINISI, 1999).

Como parte de aplicações em controle de processos em geral, e em particular como um elemento integrante de sistemas mecatrônicos, o sistema de visão computacional representa um sensor de posição baseado na reconstrução de cenas a partir imagens. Um sistema mecatrônico é um sistema mecânico onde se procura suprir limitações ou simplificar projetos mecânicos através da utilização do controle (ACAR M.; PARKIN, 1996) - e assim dependem fundamentalmente da capacidade de coletar e analisar informações relevantes do ambiente ao seu redor. Um robô autônomo é um sistema mecatrônico capaz de realizar tarefas em um ambiente desestruturado sem supervisão humana contínua. Variados tipos de robôs têm variados graus de autonomia, que se apresenta de formas também variadas. Uma importante área de pesquisa em robótica é aquela que procura trazer um alto grau de autonomia aos robôs com relação ao seu ambiente de operação, seja ele a terra, o espaço, o ar, subterrâneos ou submarino. Neste sentido, dentre os diversos transdutores utilizados para interação entre um sistema mecatrônico e o ambiente circundante destacam-se os sistemas de visão computacional. Como hardware, são compostos basicamente de câmeras - com variados graus de tamanho, recursos e resolução. Como software, são compostos de um conjunto de algoritmos que realizam tarefas variadas, como reconhecimento de objetos, tracking, reconstrução de cenas e estimativa de posição, interligados por um sistema de controle ou de inteligência artificial, que podem inclusive ser externos. Em ambos os casos, este sensor de visão representa um sistema altamente versátil e modular, facilmente modificável, onde a um custo de diferentes níveis de com- 
plexidade de algoritmos computacionais pode-se obter informações de maior ou menor precisão e abrangência. Estas características, conforme apontado por Acar (ACAR M.; PARKIN, 1996), é um dos objetivos da mecatrônica: o desenvolvimento de sistemas com inteligência e flexibilidade.

\subsection{Justificativa}

Como mencionado na seção anterior, os sistemas mecatrônicos - e em especial os sistemas robóticos autônomos - são sistemas mecânicos capazes de realizar tarefas específicas sem a intervenção humana, operando através de sofisticados sistemas de controle. Atualmente, dado ao elevado grau de complexidade requerido por um sistema realmente autônomo, a maioria das aplicações deve ser classificada como semi-autônoma. Dentre estas, é de especial interesse a classe dos ROVs - do inglês "Remotely Operated Vehicles" - cuja utilização em locais perigosos ou inacessíveis motiva o seu contínuo aperfeiçoamento. Normalmente conectados a um navio através de um "cordão umbilical" - um feixe de cabos que provêem alimentação de energia e troca de sinais e dados variados de controle, existe a necessidade de trazer mais autonomia a estes equipamentos para que possam atender a uma demanda crescente de aplicações. Como o ambiente marinho é altamente reflexivo, existe grande dificuldade na utilização de dispositivos de comunicação sem fio, levando à necessidade de embarcar os sistemas de controle e tomada de decisão a fim de se alcançar a autonomia desejada. Neste sentido, vários trabalhos procurando utilizar sistemas de visão em ROVs foram desenvolvidos recentemente, em aplicações como manipulação (MARANI; YUH, 2006), estabilização e navegação (MARTINS et al., 2006), (CACCIA, 2006). Um panorama geral das pesquisas recentes de visão aplicada ao controle de veículos submarinos autônomos pode ser visto no artigo de Horgan e Toal (HORGAN; TOAL, 2006), mostrando as tendências atuais deste campo. Assim, como parte de um projeto de construção de um robô autônomo móvel submarino, deseja-se implementar um sensor de visão com características de baixo consumo de energia, flexibilidade e inteligência. Em termos de hardware, uma câmera adapta-se perfeitamente a este objetivo. Em termos de software, a escolha são os algoritmos de reconstrução de cena da visão computacional.

Dentro do projeto de um sensor de visão versátil baseado em visão computacional, os algoritmos responsáveis pela estimação de posição fornecem as informações repassadas ao sistema de controle global do robô autônomo. Assim, é importante quantificar o erro inerente em tais medições, paralelamente ao cus- 
to de processamento dos métodos envolvidos. Dentre as variadas abordagens da visão computacional, a geometria epipolar destaca-se por fornecer a reconstrução de cenas a partir de apenas duas imagens, com métodos extensamente pesquisados na última década. Este método é composto de várias etapas encadeadas, e o erro introduzido em cada etapa influencia o resultado da etapa seguinte, sendo necessário analisar o efeito deste encadeamento no resultado da estimação de posição. A complexidade computacional de cada algoritmo em cada uma das etapas do processamento também é um fator importante na escolha do melhor método de reconstrução de cena, pois o hardware de um ROV é limitado por questões de carga, volume e consumo de energia, e como tal, deve ser analisada em conjunto com o erro.

Apesar dos vários trabalhos recentes sobre utilização de sistemas de visão em ROVs mencionados anteriormente, todos a utilizam de forma específica, dedicada a tarefas bem determinadas, e ainda há pouca preocupação com o aspecto metrológico, de avaliação de erros. Assim, este trabalho foi desenvolvido para fornecer uma base teórica e uma análise comparativa de resultados práticos da estimação de posição através da geometria epipolar entre imagens, através do erro e da complexidade computacional, como parte da implementação de um sensor de visão de para robôs autônomos.

\subsection{Objetivos}

O objetivo deste trabalho é realizar uma análise comparativa de algoritmos de estimação de posição por geometria epipolar. Para tanto foi realizado um levantamento da teoria envolvida nestes algoritmos, um estudo quantitativo do erro inerente e da complexidade computacional destes métodos, e a análise de dados e resultados da aplicação dos mesmos em simulações e montagens experimentais. A compilação teórica fornece uma base detalhada e compreensível dos conceitos necessários para implementar um algoritmo de estimação de posição através de geometria epipolar, entender e interpretar corretamente os resultados observados, e analisar os componentes do erro presente em medições destes resultados. A parte prática deste trabalho apresenta a implementação dos algoritmos analisados na parte teórica, e a análise dos resultados quanto à acuidade das medições e complexidade de execução, simulando os movimentos especiais de câmera estudados teoricamente por meio de imagens geradas por computador, e emulando o movimento genérico de câmera por meio de montagens experimentais em laboratório. A análise dos resultados práticos em face dos conceitos teóricos permite 
formar um comparativo dos métodos de estimação de posição utilizando geometria epipolar quanto ao erro e complexidade, e também determinar as situações em que melhor se aplicam e os pré-requisitos necessários para obtenção dos melhores resultados.

\subsection{Organização}

A pesquisa realizada neste trabalho foi dividida em duas partes principais: apresentação de conceitos teóricos e análise de resultados práticos.

Os conceitos teóricos abordados são: geometria projetiva, modelo de câmera, geometria epipolar, matriz fundamental, reconstrução projetiva, reconstrução métrica, algoritmos de determinação da matriz fundamental, algoritmos de reconstrução métrica, incerteza da matriz fundamental e complexidade computacional. Os conceitos de geometria projetiva, modelo de câmera, geometria epipolar, matriz fundamental, reconstrução projetiva e reconstrução métrica formam uma base para compreensão dos conceitos de algoritmos de determinação da matriz fundamental e de reconstrução métrica. Os conceitos de incerteza da matriz fundamental e de complexidade computacional permitem comparar os resultados destes algoritmos. Os tópicos foram detalhados nos capítulos da parte teórica deste trabalho de forma a fornecer um tutorial para o estudo da estimação de posição e quantificação do erro utilizando geometria epipolar entre imagens. A parte teórica permite elaborar a análise dos resultados e apresentar as conclusões ao final deste trabalho.

Os resultados práticos apresentados na segunda parte deste trabalho baseiamse na implementação dos algoritmos estudados na parte teórica e sua aplicação em laboratório. Foram desenvolvidos algoritmos de determinação da matriz fundamental e de reconstrução métrica de cenas, bem como rotinas de processamento para a determinação do erro em diversos estágios do processo. Todos os algoritmos foram desenvolvidos em MATLAB ${ }^{\circledR}$ 6.5, linguagem de alto desempenho voltada ao cálculo numérico e matricial, amplamente utilizada em aplicações científicas e de engenharia. Desta forma, os erros analisados podem ser considerados relativos apenas ao processo de estimação de posição, uma vez que a implementação já é otimizada para os cálculos envolvidos. Os dados processados por estes algoritmos foram obtidos de imagens geradas por computador simulando movimentos especiais de câmera, e de montagens experimentais em ambiente de laboratório emulando o movimento geral de câmera. As simulações com imagens geradas por computador também foram criadas utilizando MATLAB ${ }^{\circledR}$, e fornecem um con- 
junto de dados cujas medidas são livres de ruído e permitem verificar a eficácia de cada algoritmo estudado. As montagens experimentais utilizam uma câmera digital acoplada a um robô programável, capaz de realizar movimentos de translação e rotação em relação às três dimensões do espaço. Desta forma, pode-se simular o movimento com o número de graus de liberdade equivalente ao de um ROV em ambiente submarino. Este trabalho apresenta três montagens experimentais: uma primeira montagem, complementar à simulação com imagens geradas por computador; uma montagem experimental realizando apenas movimento planar paralelo ao plano de imagem; e uma montagem experimental realizando movimentos de câmera variados em seqüência, simulando a movimentação de um sistema móvel sem restrições de rotação e translação e com velocidade variável. Os dados das imagens geradas por computador e da primeira montagem experimental foram utilizados para comparar o desempenho dos algoritmos de determinação da matriz fundamental, a base do algoritmo de estimação de posição. As duas outras montagens experimentais foram utilizadas para analisar os resultados da reconstrução métrica e determinar as condições de utilização do mesmo.

Assim, este trabalho foi organizado segundo a seguinte estrutura:

1. Conceitos Teóricos

(a) Geometria Projetiva (Capítulo 2)

(b) Modelo de Câmera (Capítulo 3)

(c) Geometria Epipolar e Matriz Fundamental (Capítulo 4)

(d) Cálculo da Matriz Fundamental (Capítulo 5)

(e) Incerteza da Matriz Fundamental (Capítulo 6)

(f) Reconstrução Métrica (Capítulo 7)

(g) Complexidade computacional (Capítulo 8)

2. Resultados Práticos

(a) Conjuntos de Dados (Capítulo 9, Seção 9.1)

(b) Processamento (Capítulo 9, Seção 9.2)

(c) Análise (Capítulo 9, Seção 9.3)

3. Conclusão (Capítulo 9.3.3)

Alguns conceitos teóricos adicionais que visam a melhor compreensão de alguns tópicos da primeira parte foram colocados no Apêndice A, e os algoritmos em MATLAB ${ }^{\circledR} 6.5$ estão listados no Apêndice B, ao final deste trabalho. 
Parte I

Conceitos Teóricos 


\section{Geometria Projetiva}

Este capítulo apresenta os conceitos básicos de Geometria Projetiva, que fornece a representação matemática, a terminologia e a notação necessários para a compreensão dos demais conceitos teóricos apresentados neste trabalho. Inicialmente são apresentados os conceitos relativos a transformações projetivas do espaço bidimensional, que posteriormente são estendidos para a geometria projetiva do espaço tridimensional. Tais conceitos são melhor visualizados no espaço 2D que em 3D, e a geometria projetiva do espaço tridimensional é uma simples generalização do caso bidimensional.

A geometria é basicamente o estudo da relação entre pontos e retas, e pode ser formulada de forma puramente geométrica ou de forma algébrica. No primeiro caso, teoremas baseiam-se apenas em primitivas geométricas, e no segundo em coordenadas e entidades algébricas. Dessa forma, pontos e retas são identificados como vetores, e cônicas são representadas por matrizes. A abordagem geométrica permite a visualização dos conceitos, enquanto a abordagem algébrica fornece meios de implementar algoritmos e desenvolver métodos computacionais.

Os conceitos apresentados neste capítulo foram extraídos de (HARTLEY; ZISSERMAN, 2000), (FAUGERAS, 1993), (ULlMAN, 1979), (SEMPLE; KNEEBONE, 1952) e (XU; ZHANG, 1996). A notação adotada e as ilustrações das figuras são de (HARTLEY; ZISSERMAN, 2000).

\subsection{Geometria Projetiva 2D}

Um ponto pertencente a um plano pode ser representado por um par de coordenadas $(x, y)$ de $\mathbf{R}^{\mathbf{2}}$. Como transformações lineares entre espaços vetoriais são representadas por matrizes, é conveniente representar as entidades geométricas por vetores coluna. Assim, o ponto passa a ser representado pelo vetor $\mathbf{x}=(x, y)^{\mathrm{T}}$ do espaço vetorial $\mathbf{R}^{2}$.

Representação homogênea de retas. A representação natural da reta é 
o vetor $(a, b, c)^{\mathrm{T}}$, correspondente à equação $a x+b y+c=0$, e todos os vetores equivalentes $k(a, b, c)^{\mathrm{T}}$ representam a mesma reta, para qualquer escalar $k$ não nulo. Uma classe de vetores equivalentes sob esta relação é conhecida como um vetor homogêneo, e qualquer vetor $k(a, b, c)^{\mathrm{T}}$ é um representante desta classe de equivalência. O conjunto de classes de equivalência de vetores em $\mathbf{R}^{\mathbf{3}}-(0,0,0)^{\mathrm{T}}$ forma o espaço projetivo $\mathbf{P}^{2}$.

Representação homogênea de pontos. Um ponto $\mathbf{x}=(x, y)^{\mathrm{T}}$ pertence à reta $\mathbf{l}=(a, b, c)^{\mathrm{T}}$ se e somente se $a x+b y+c=0$. Em termos do produto interno, Um ponto $\mathbf{x}$ pertence à reta $\mathbf{l}$ se e somente se $\mathbf{x}^{\mathrm{T}} \mathbf{l}=0$. Como para qualquer escalar $k$ não nulo tem-se $k(x, y, 1) \mathbf{l}=0$, então o ponto $(x, y)^{\mathrm{T}}$ em $\mathbf{R}^{\mathbf{2}}$ pode ser representado por um vetor homogêneo. Dessa forma, um vetor homogêneo qualquer $\mathbf{x}=\left(x_{1}, x_{2}, x_{3}\right)^{\mathrm{T}}$ representa o ponto $\left(x_{1} / x_{3}, x_{2} / x_{3}\right)^{\mathrm{T}}$ em $\mathbf{R}^{2}$.

Intersecção de duas retas. A intersecção de duas retas $\mathbf{l}$ e $\mathbf{l}^{\prime}$ é o ponto $\mathbf{x}=\mathbf{l} \times \mathbf{l}^{\prime}$. Dadas duas retas $\mathbf{l}=(a, b, c)^{\mathrm{T}}$ e $\mathbf{l}^{\prime}=\left(a^{\prime}, b^{\prime}, c^{\prime}\right)^{\mathrm{T}}$, o produto vetorial $\mathbf{x}=\mathbf{l} \times \mathbf{l}^{\prime}$ é tal que $\mathbf{l}^{\mathrm{T}} \mathbf{x}=\mathbf{l}^{\prime \mathrm{T}} \mathbf{x}=0$. Assim, o ponto $\mathbf{x}$ pertence a ambas retas $\mathbf{l} \mathrm{e}$ $\mathbf{l}^{\prime}$ e representa a intersecção entre elas.

Reta definida por dois pontos. Analogamente ao caso da intersecção de duas retas, A reta que passa pelos pontos $\mathbf{x}$ e $\mathbf{x}^{\prime}$ é $\mathbf{l}=\mathbf{x} \times \mathbf{x}^{\prime}$.

Intersecção de retas paralelas. A intersecção entre as retas $\mathbf{l}=(a, b, c)^{\mathrm{T}}$ e $\mathbf{l}^{\prime}=\left(a, b, c^{\prime}\right)^{\mathrm{T}}$ é $\mathbf{l} \times \mathbf{l}^{\prime}=\left(c^{\prime}-c\right)(b,-a, 0)^{\mathrm{T}}$. O ponto $(b,-a, 0)^{\mathrm{T}}$ não representa nenhum ponto finito em $\mathbf{R}^{2}$, e corresponde à definição geométrica de duas retas paralelas que se cruzam no infinito.

Pontos ideais e reta no infinito. Vetores homogêneos $\mathbf{x}=\left(x_{1}, x_{2}, x_{3}\right)^{\mathrm{T}}$ de coordenada $x_{3} \neq 0$ correspondem a pontos em $\mathbf{R}^{2}$. Acrescentando a $\mathbf{R}^{2}$ os pontos de coordenada $x_{3}=0$, tem-se o conjunto de todos os vetores homogêneos de 3 dimensões, o espaço projetivo $\mathbf{P}^{2}$. Os pontos com coordenada $x_{3}=0$ são chamados pontos ideais, ou pontos no infinito. O conjunto de todos pontos ideais pode ser escrito $\left(x_{1}, x_{2}, 0\right)^{\mathrm{T}}$, e todos estes pontos pertencem a uma reta, a reta no infinito, denotada $\mathbf{l}_{\infty}=(0,0,1)^{\mathrm{T}}$.

Uma reta $\mathbf{l}=(a, b, c)^{\mathrm{T}}$ cruza a reta $\mathbf{l}_{\infty}$ no ponto ideal $(b,-a, 0)^{\mathrm{T}}$, assim como qualquer reta $\mathbf{l}^{\prime}=\left(a, b, c^{\prime}\right)^{\mathrm{T}}$ paralela a $\mathbf{l}$. Dessa forma, o ponto ideal $(b,-a, 0)^{\mathrm{T}}$ representa a direção da reta $\mathbf{l}=(a, b, c)^{\mathrm{T}}$, e a reta no infinito $\mathbf{l}_{\infty}$ representa o conjunto de direções de retas em um plano.

Modelo do plano projetivo. O espaço projetivo $\mathbf{P}^{2}$ consiste em um conjunto de raios em $\mathbf{R}^{3}$, uma vez que o conjunto de todos pontos $k\left(x_{1}, x_{2}, x_{3}\right)^{\mathrm{T}}$ 


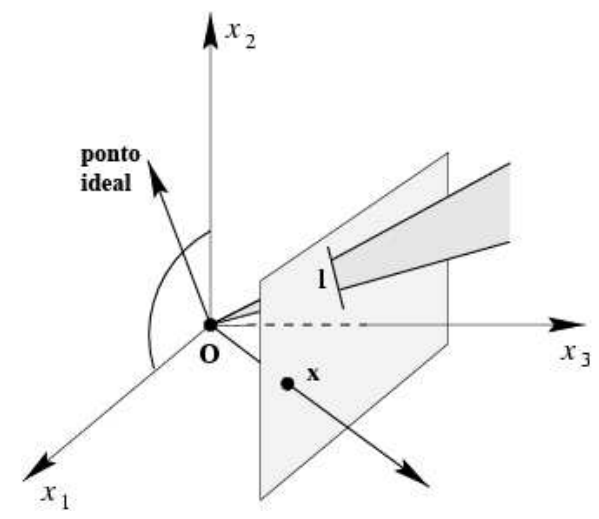

Figura 2.1: Modelo do plano projetivo.

forma raios passando pela origem a medida que varia-se $k$, e cada um destes raios representa um único ponto em $\mathbf{P}^{2}$. Neste modelo, retas de $\mathbf{P}^{\mathbf{2}}$ representam planos passando pela origem em $\mathbf{R}^{\mathbf{3}}$. Os raios representando pontos ideais e o plano representando $\mathbf{l}_{\infty}$ são paralelos ao plano $x_{3}=0$, conforme ilustrado na Figura 2.1.

Princípio da dualidade. No espaço projetivo $\mathbf{P}^{\mathbf{2}}$, os papéis de pontos e retas podem ser intercambiados com relação a suas propriedades. O princípio da dualidade estabelece que a qualquer teorema da geometria projetiva do espaço bidimensional corresponde um teorema dual, derivado permutando-se os papéis de pontos e retas no teorema original.

Cônicas. Uma cônica é uma curva representada por uma equação de segundo grau no plano. Na Geometria Euclidiana, as cônicas são de três tipos: hipérboles, elipses e parábolas. Estes três tipos de cônicas derivam-se das seções cônicas geradas pela intersecção com planos em diferentes orientações. No entanto, no espaço projetivo 2D todas cônicas são equivalentes sob transformações projetivas. A equação de uma cônica em função das coordenadas homogêneas do ponto $\mathbf{x}=$ $\left(x_{1}, x_{2}, x_{3}\right)^{\mathrm{T}}$ é o polinômio de segundo grau $a x_{1}^{2}+b x_{1} x_{2}+c x_{2}^{2}+d x_{1} x_{3}+e x_{2} x_{3}+f x_{3}^{2}=$ 0. Em notação matricial, tem-se:

$$
\mathrm{x}^{\mathrm{T}} \mathrm{Cx}=0
$$

onde a matriz $\mathrm{C}$ de coeficientes da cônica é dada por:

$$
\mathrm{C}=\left[\begin{array}{ccc}
a & b / 2 & d / 2 \\
b / 2 & c & e / 2 \\
d / 2 & e / 2 & f
\end{array}\right]
$$

Reta tangente a uma cônica. Em coordenadas homogêneas a reta $\mathbf{l}$ tan- 


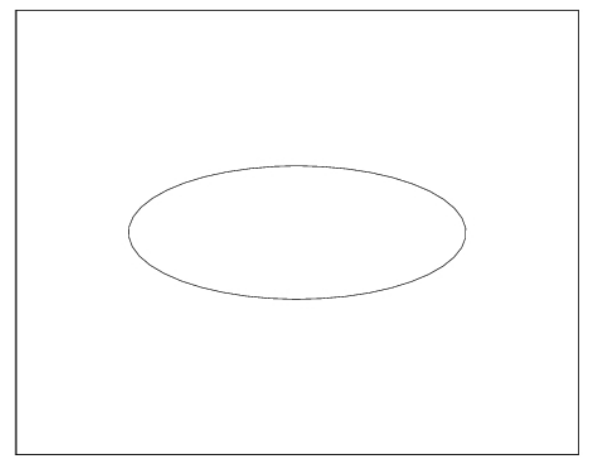

(a)

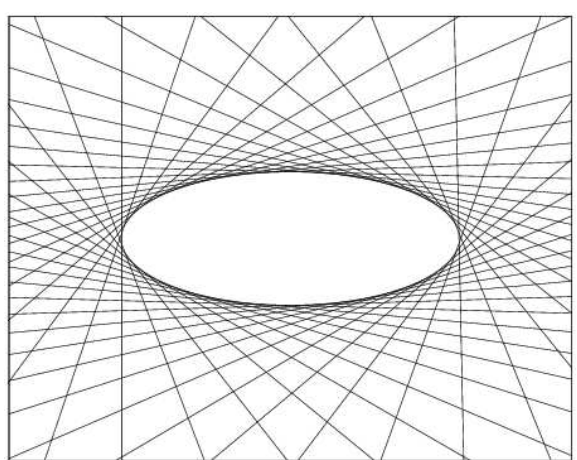

(b)

Figura 2.2: Exemplo de (a) cônica e (b) cônica dual.

gente à cônica $\mathrm{C}$ no ponto $\mathbf{x}$ é dada pelo produto matricial $\mathbf{l}=\mathbf{C x}$.

Cônicas duais. A cônica C de (2.2) é denominada uma cônica pontual, pois define uma equação sobre pontos. Dada a natureza dual de $\mathbf{P}^{\mathbf{2}}$, existe uma cônica dual, ou cônica linear, que define uma equação sobre retas. Uma reta 1 tangente à cônica $C$ satisfaz a equação $\mathbf{l}^{\mathrm{T}} \mathrm{C}^{*} \mathbf{l}=0$, onde $\mathrm{C}^{*}$ é a adjunta de $\mathrm{C}$ (transposta conjugada), e denota sua cônica dual. Cônicas duais também são chamadas de envelopes cônicos, conforme ilustrado na Figura 2.2. A Figura 2.2a mostra os pontos $\mathbf{x}$ tais que $\mathbf{x}^{\mathrm{T}} \mathbf{C x}=0$, definindo uma cônica pontual. A Figura 2.2b mostra as retas $\mathbf{l}$ tais que $\mathbf{l}^{\mathrm{T}} \mathrm{C}^{*} \mathbf{l}=0$, tangentes à cônica pontual $\mathrm{C}$, representado o envelope de retas de $\mathrm{C}$.

Cônicas degeneradas. Se a matriz C de (2.2) não possui posto completo, ela representa uma cônica degenerada. Geometricamente, cônicas degeneradas ocorrem quando o plano secionante contém o vértice do cone. Cônicas degeneradas pontuais incluem duas retas (posto 2), e uma reta (posto 1). Cônicas degeneradas lineares incluem dois pontos (posto 2) e um ponto (posto 1).

Transformações projetivas. A geometria projetiva 2D é o estudo das propriedades de $\mathbf{P}^{\mathbf{2}}$ invariantes sob um grupo de transformações chamadas projetividades. Um mapa $h: \mathbf{P}^{\mathbf{2}} \rightarrow \mathbf{P}^{\mathbf{2}}$ é uma projetividade se e somente se existe uma matriz não-singular H $3 \times 3$ tal que para qualquer ponto $\mathbf{x}$ em $\mathbf{P}^{2}$ vale a relação $h(\mathbf{x})=\mathrm{Hx}$. As projetividades formam um grupo pois a inversa de uma projetividade e a composição de duas projetividades também é uma projetividade. Uma projetividade também é chamada de colineação, transformação projetiva ou homografia.

Uma transformação projetiva planar é uma transformação linear de vetores homogêneos de 3 dimensões representada por uma matriz H $3 \times 3$ não-singular, 
tal que $\mathbf{x}^{\prime}=\mathrm{Hx}$ :

$$
\left(\begin{array}{l}
x_{1}^{\prime} \\
x_{2}^{\prime} \\
x_{3}^{\prime}
\end{array}\right)=\left[\begin{array}{lll}
h_{11} & h_{12} & h_{13} \\
h_{21} & h_{22} & h_{23} \\
h_{31} & h_{32} & h_{33}
\end{array}\right]\left(\begin{array}{l}
x_{1} \\
x_{2} \\
x_{3}
\end{array}\right) .
$$

A matriz H é dita uma matriz homogênea pois pode ser multiplicada por um fator de escala não-nulo sem alterar a transformação projetiva. Somente as 8 razões independentes entre os 9 elementos da matriz são significativas, e portanto H tem 8 graus de liberdade.

Alguns casos particulares de transformações projetivas formam subgrupos dentro do grupo projetivo linear, que podem ser organizados em uma hierarquia de classes de transformações, cada qual representando uma generalização da classe anterior. Esta hierarquia é formada pelas transformações isométrica, similar, afim e projetiva. Cada uma destas transformações pode ser representada pelos seus invariantes: função escalar da configuração geométrica cujo valor não é alterado pela transformação.

As transformações isométricas, ou isometrias, são transformações do plano $\mathbf{R}^{\mathbf{2}}$ que preservam a distância Euclidiana. Uma isometria é uma transformação da forma:

$$
\left(\begin{array}{l}
x^{\prime} \\
y^{\prime} \\
1
\end{array}\right)=\left[\begin{array}{ccc}
\varepsilon \cos \theta & -\sin \theta & t_{x} \\
\varepsilon \sin \theta & \cos \theta & t_{y} \\
0 & 0 & 1
\end{array}\right]\left(\begin{array}{l}
x \\
y \\
1
\end{array}\right)
$$

onde $\varepsilon= \pm 1$. Se $\varepsilon=1$, a isometria preserva a orientação e é uma transformação Euclidiana - a composição de uma rotação e uma translação. As transformações Euclidianas modelam o movimento de objetos rígidos, que pode ser escrita como:

$$
\mathbf{x}^{\prime}=\mathrm{H}_{\mathrm{E}} \mathbf{x}=\left[\begin{array}{cc}
\mathrm{R} & \mathbf{t} \\
\mathbf{0}^{\mathrm{T}} & 1
\end{array}\right] \mathbf{x},
$$

onde $\mathrm{R}$ é uma matriz de rotação ${ }^{1} 2 \times 2$, $\mathbf{t}$ é um vetor de translação de 2 dimensões, e $\mathbf{0}$ é um vetor nulo de 2 dimensões. Uma transformação Euclidiana também é chamada de deslocamento.

Uma transformação Euclidiana planar tem três graus de liberdade e seus invariantes são: comprimento (distância entre dois pontos), ângulo (ângulo entre

\footnotetext{
${ }^{1}$ matriz ortogonal tal que $\mathrm{R}^{\mathrm{T}} \mathrm{R}=\mathrm{RR}^{\mathrm{T}}=\mathrm{I}$
} 
duas retas) e área.

As transformações similares, ou similaridades, representam uma isometria composta com escalonamento isotrópico. No caso de uma transformação Euclidiana composta com um escalonamento, tem-se:

$$
\left(\begin{array}{l}
x^{\prime} \\
y^{\prime} \\
1
\end{array}\right)=\left[\begin{array}{ccc}
s \cos \theta & -s \sin \theta & t_{x} \\
s \sin \theta & s \cos \theta & t_{y} \\
0 & 0 & 1
\end{array}\right]\left(\begin{array}{l}
x \\
y \\
1
\end{array}\right) .
$$

Esta representação matricial pode ser escrita como:

$$
\mathbf{x}^{\prime}=\mathrm{H}_{\mathrm{S}} \mathbf{x}=\left[\begin{array}{ll}
s \mathrm{R} & \mathbf{t} \\
\mathbf{0}^{\mathrm{T}} & 1
\end{array}\right] \mathbf{x},
$$

onde o valor escalar $s$ representa o escalonamento isotrópico. A transformação similar também é chamada de transformação equiforme, pois preserva os contornos de figuras geométricas. Uma transformação similar planar tem 4 graus de liberdade e seus invariantes são: ângulo, razão entre duas distâncias e razão entre duas áreas.

A transformação afim, ou afinidade, representa uma transformação linear não-singular seguida de translação, com representação matricial:

$$
\left(\begin{array}{c}
x^{\prime} \\
y^{\prime} \\
1
\end{array}\right)=\left[\begin{array}{ccc}
a_{11} & a_{12} & t_{x} \\
a_{21} & a_{22} & t_{y} \\
0 & 0 & 1
\end{array}\right]\left(\begin{array}{l}
x \\
y \\
1
\end{array}\right),
$$

ou ainda:

$$
\mathbf{x}^{\prime}=\mathrm{H}_{\mathrm{A}} \mathbf{x}=\left[\begin{array}{cc}
\mathrm{A} & \mathbf{t} \\
\mathbf{0}^{\mathrm{T}} & 1
\end{array}\right] \mathbf{x},
$$

onde A é uma matriz $2 \times 2$ não-singular. Esta matriz A pode ser decomposta em uma rotação e um escalonamento anisotrópico:

$$
\mathrm{A}=\mathrm{R}(\theta) \mathrm{R}(-\phi) \mathrm{DR}(-\phi),
$$

onde $\mathrm{R}(\theta)$ e $\mathrm{R}(\phi)$ são respectivamente rotações de $\theta$ e $\phi$, e $\mathrm{D}=\operatorname{diag}\left(\lambda_{1}, \lambda_{2}\right)$. A Figura 2.3(a) ilustra o efeito da rotação $R(\theta)$, e a Figura 2.3(b) mostra a deformação $\mathrm{R}(-\phi) \operatorname{DR}(-\phi)$. Nota-se que as direções de escalonamento na deformação são or- 


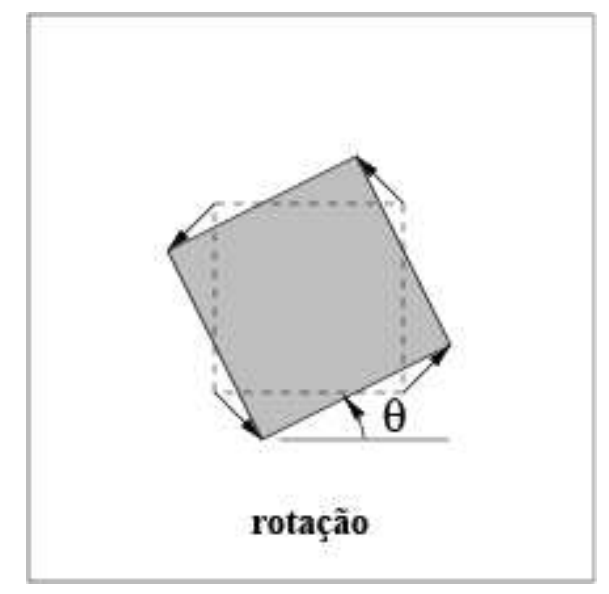

(a)

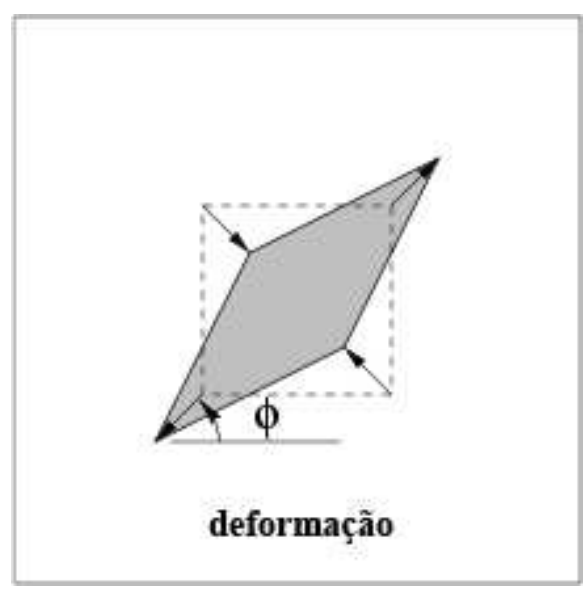

(b)

Figura 2.3: Distorção causada pela transformação afim planar. (a) Rotação. (b) Deformação.

togonais.

Uma transformação afim planar tem seis graus de liberdade e seus invariantes são: paralelismo, razão entre os comprimentos de dois segmentos de reta paralelos, e razão entre duas áreas.

O caso geral da transformação projetiva definida em (2.3), seguindo a mesma representação dos casos particulares anteriores, pode ser escrita como:

$$
\mathbf{x}^{\prime}=\mathrm{H}_{\mathrm{P}} \mathbf{X}=\left[\begin{array}{cc}
\mathrm{A} & \mathbf{t} \\
\mathbf{v}^{\mathrm{T}} & v
\end{array}\right] \mathbf{x},
$$

onde $\mathbf{v}=\left(v_{1}, v_{2}\right)^{\mathrm{T}}$ e $v$ é um escalar. A matriz homogênea $\mathrm{H}_{\mathrm{P}}$ tem nove elementos cujas oito razões independentes correspondem aos 8 graus de liberdade da transformação projetiva, e seu principal invariante é a razão cruzada entre quatro pontos colineares (razão entre razões de comprimentos sobre uma reta).

A Tabela 2.1 mostra os grupos de transformações 2D e seus invariantes, onde $\mathrm{A}=\left[a_{i j}\right]$ é uma matriz $2 \times 2$ inversível, $\mathrm{R}=\left[r_{i j}\right]$ é uma matriz de rotação $2 \mathrm{D}$, e $\mathbf{t}=\left(t_{x}, t_{y}\right)^{\mathrm{T}}$ é uma translação, onde a transformação de uma linha representa uma generalização da transformação da linha imediatamente inferior e um caso particular da transformação da linha imediatamente superior, da qual herda todos os invariantes.

Uma transformação projetiva pode ser decomposta em uma cadeia de transformações da hierarquia descrita anteriormente, na ordem crescente de generalização: 


$$
\mathrm{H}=\mathrm{H}_{\mathrm{S}} \mathrm{H}_{\mathrm{A}} \mathrm{H}_{\mathrm{P}}=\left[\begin{array}{ll}
\mathbf{s R} & \mathbf{t} \\
\mathbf{0}^{\mathrm{T}} & 1
\end{array}\right]\left[\begin{array}{cc}
\mathrm{K} & \mathbf{t} \\
\mathbf{0}^{\mathrm{T}} & 1
\end{array}\right]\left[\begin{array}{cc}
\mathrm{I} & \mathbf{0} \\
\mathbf{v}^{\mathrm{T}} & v
\end{array}\right]=\left[\begin{array}{cc}
\mathrm{A} & \mathbf{t} \\
\mathbf{v}^{\mathrm{T}} & v
\end{array}\right] .
$$

Pontos circulares e cônica absoluta. Os pontos circulares, também chamados pontos absolutos, $\mathbf{I}=(1, i, 0)^{\mathrm{T}}$ e $\mathbf{J}=(1,-i, 0)^{\mathrm{T}}$, representam os pontos onde todas circunferências cruzam a reta no infinito $\mathbf{l}_{\infty}$. Os pontos circulares $\mathbf{I}, \mathbf{J}$ são fixos sob a transformação projetiva $\mathrm{H}$ se e somente se $\mathrm{H}$ for uma similaridade.

A cônica dual aos pontos circulares é dada por:

$$
\mathrm{C}_{\infty}^{*}=\mathbf{I} \mathbf{J}^{\mathrm{T}}+\mathbf{J I}^{\mathrm{T}}
$$

e representa uma cônica linear degenerada (posto 2). A cônica dual $\mathrm{C}_{\infty}^{*}$ é fixa sob a transformação projetiva $\mathrm{H}$ se e somente se $\mathrm{H}$ for uma similaridade.

A cônica dual $\mathrm{C}_{\infty}^{*}$ tem 4 graus de liberdade. $\mathrm{O}$ vetor nulo da cônica dual $\mathrm{C}_{\infty}^{*}$ é a reta no infinito $\mathbf{l}_{\infty}$. Assim, o ângulo entre duas retas $\mathbf{l}$ e $\mathbf{m}$ é dado por:

$$
\cos \theta=\frac{\mathbf{l}^{\mathrm{T}} C_{\infty}^{*} \mathbf{m}}{\sqrt{\left(\mathbf{l}^{\mathrm{T}} C_{\infty}^{*} \mathbf{l}\right)\left(\mathbf{l}^{\mathrm{T}} C_{\infty}^{*} \mathbf{m}\right)}}
$$

Adicionalmente, duas retas $\mathbf{l}$ e $\mathbf{m}$ são ortogonais se $\mathbf{l}^{\mathrm{T}} \mathrm{C}_{\infty}^{*} \mathbf{m}=0$.

\subsection{Geometria Projetiva 3D}

Muitas das propriedades e entidades do espaço projetivo $3 \mathrm{D}$, ou $\mathbf{P}^{\mathbf{3}}$ são generalizações diretas das suas equivalentes no plano projetivo, descritas na Seção 2.1. Em $\mathbf{P}^{3}$, o espaço Euclidiano tridimensional é ampliado por um conjunto de pontos ideais pertencentes a um plano no infinito, $\pi_{\infty}$, análogo a $\mathbf{l}_{\infty}$ em $\mathbf{P}^{2}$. Retas paralelas em $\mathbf{P}^{\mathbf{2}}$ correspondem a planos paralelos em $\mathbf{P}^{\mathbf{3}}$, que se cruzam em uma reta sobre $\pi_{\infty}$. Apesar destas semelhanças, as coordenadas homogêneas em $\mathbf{P}^{\mathbf{3}}$ são representadas por vetores de quatro dimensões, introduzindo propriedades adicionais em relação a $\mathbf{P}^{2}$.

Pontos. A representação homogênea de um ponto $\mathbf{X}$ do espaço tridimensional é um vetor de 4 dimensões. Dado o vetor homogêneo $\mathbf{X}=\left(x_{1}, x_{2}, x_{3}, x_{4}\right)^{\mathrm{T}}$, com $x_{4} \neq 0$, o ponto $(\mathrm{X}, \mathrm{Y}, \mathrm{Z})^{\mathrm{T}}$ correspondente em $\mathbf{R}^{\mathbf{3}}$ tem coordenadas nãohomogêneas $\mathrm{X}=x_{1} / x_{4}, \mathrm{Y}=x_{2} / x_{4}$ e $\mathrm{Z}=x_{3} / x_{4}$. Pontos homogêneos com a coordenada $x_{4}=0$ representam pontos no infinito. 
Transformações Projetivas. Uma transformação projetiva em $\mathbf{P}^{\mathbf{3}}$ é uma transformação linear sobre vetores homogêneos de 4 dimensões, e é representada por uma matriz H $4 \times 4$ não-singular tal que $\mathbf{X}^{\prime}=\mathrm{HX}$. A matriz H é homogênea e tem 15 graus de liberdade. Como no caso das transformações projetivas planares, o mapa é uma colineação (retas são transformadas em retas), que preserva relações como o ponto de intersecção entre uma reta e um plano e ordem de contato.

Dualidade. Em $\mathbf{P}^{3}$ pontos e planos são duais, com representações e propriedades análogas às da dualidade entre ponto e reta em $\mathbf{P}^{2} \cdot \operatorname{Em} \mathbf{P}^{\mathbf{3}}$, retas são auto-duais.

Planos. Um plano do espaço tridimensional pode ser equacionado como $\pi_{1} \mathrm{X}+\pi_{2} \mathrm{Y}+\pi_{3} \mathrm{Z}+\pi_{4}=0$. A representação homogênea de um plano é o vetor de 4 dimensões $\pi=\left(\pi_{1}, \pi_{2}, \pi_{3}, \pi_{4}\right)^{\mathrm{T}}$ e as três razões independentes $\left\{\pi_{1}: \pi_{2}: \pi_{3}: \pi_{4}\right\}$ definem os três graus de liberdade do plano no espaço tridimensional. Utilizando a representação homogênea do ponto $\mathbf{X}=\left(x_{1}, x_{2}, x_{3}, x_{4}\right)^{\mathrm{T}}$ tem-se $\pi_{1} x_{1}+\pi_{2} x_{2}+$ $\pi_{3} x_{3}+\pi_{4} x_{4}=0$. Assim, a expressão do ponto $\mathbf{X}$ sobre o plano $\pi$ é:

$$
\pi^{\mathrm{T}} \mathbf{X}=0 .
$$

O vetor $\mathbf{n}=\left(\pi_{1}, \pi_{2}, \pi_{3}\right)^{\mathrm{T}}$ representa a normal ao plano em $\mathbf{R}^{\mathbf{3}}$.

Plano definido por três pontos. Dados três pontos $\mathbf{X}_{i}, i=1, \ldots, 3$ pertencentes a um plano $\pi$, por (2.15) tem-se:

$$
\left[\begin{array}{l}
\mathbf{X}_{1}{ }^{\mathrm{T}} \\
\mathbf{X}_{2}{ }^{\mathrm{T}} \\
\mathbf{X}_{3}{ }^{\mathrm{T}}
\end{array}\right] \pi=\mathbf{0}
$$

A solução desta equação é dada pelo espaço nulo da matriz $3 \times 4$ formada pelos três pontos como linhas. Se os pontos $\mathbf{X}_{1}, \mathbf{X}_{2}, \mathbf{X}_{3}$ não são todos colineares, a matriz tem posto 3 , e existe uma única solução para o plano $\pi$. Se a matriz tem posto 2, os três pontos são colineares, e determinam um feixe de planos com eixo na reta formada por eles.

Ponto definido por três planos. Este é o caso dual do plano definido por três pontos. Assim, o ponto $\mathbf{X}$ definido pela intersecção de três planos $\pi_{i}$, $i=1, \ldots, 3$, é dado pelo espaço nulo da matriz $3 \times 4$ composta pelos planos como linhas: 


$$
\left[\begin{array}{l}
\pi_{1}^{\mathrm{T}} \\
\pi_{1}^{\mathrm{T}} \\
\pi_{1}^{\mathrm{T}}
\end{array}\right] \mathbf{X}=\mathbf{0} .
$$

Transformação projetiva sobre um plano. Dada uma transformação pontual $\mathbf{X}^{\prime}=\mathrm{HX}$ sobre um ponto, um plano sofre a transformação:

$$
\pi^{\prime}=\mathrm{H}^{-\mathrm{T}} \pi
$$

Pontos parametrizados sobre um plano. O ponto $\mathrm{X}$ sobre o plano $\pi$ pode ser escrito como:

$$
\mathrm{X}=\mathrm{Mx}
$$

onde matriz M $4 \times 3$ é tal que $\pi^{\mathrm{T}} \mathrm{M}=\mathbf{0}$, e o vetor de 3 dimensões $\mathbf{x}$ é um ponto no plano projetivo $\mathbf{P}^{2}$, e parametriza os pontos sobre o plano $\pi$.

Retas. Uma reta é definida como a união de dois pontos, ou a intersecção de dois planos, e tem quatro graus de liberdade no espaço tridimensional. Duas representações compatíveis com vetores homogêneos de $\mathbf{P}^{\mathbf{3}}$ pode ser derivadas da própria definição de reta: como a composição de dois pontos, ou sua versão dual como a intersecção de dois planos. Geometricamente, uma reta é um feixe de pontos colineares, definida por quaisquer dois destes pontos. Analogamente, uma reta representa o eixo de um feixe de planos, definida pela intersecção de quaisquer dois destes planos.

Dados dois pontos A, B não coincidentes, a reta unindo por estes dois pontos é representada pelo suporte do espaço vetorial das linhas da matriz $\mathrm{W} 2 \times 4$ composta por $\mathbf{A}^{\mathrm{T}}$ e $\mathbf{B}^{\mathrm{T}}$ :

$$
\mathrm{W}=\left[\begin{array}{l}
\mathbf{A}^{\mathrm{T}} \\
\mathbf{B}^{\mathrm{T}}
\end{array}\right] .
$$

Desta forma, o suporte de $\mathrm{W}^{\mathrm{T}}$ é o feixe de pontos $\lambda \mathbf{A}+\mu \mathbf{B}$ sobre a reta, e o suporte do espaço nulo de $\mathrm{W}$ é o feixe de planos cujo eixo é esta reta.

A representação dual da reta como a intersecção de dois planos, $\mathbf{P}, \mathbf{Q}$ é o suporte do espaço vetorial das linhas da matriz $\mathrm{W}^{*} 2 \times 4$ composta por $\mathbf{P}^{\mathrm{T}}$ e $\mathbf{Q}^{\mathrm{T}}$ : 


$$
\mathrm{W}=\left[\begin{array}{l}
\mathbf{P}^{\mathrm{T}} \\
\mathbf{Q}^{\mathrm{T}}
\end{array}\right] .
$$

Dessa forma, o suporte de $\mathrm{W}^{* \mathrm{~T}}$ é o feixe de planos $\lambda^{\prime} \mathbf{P}+\mu^{\prime} \mathbf{P}$ com a reta como eixo, e o suporte do espaço nulo bidimensional de $\mathrm{W}^{*}$ é o feixe de pontos sobre esta reta.

O plano $\pi$ definido pela união do ponto $\mathbf{X}$ e da reta $W$ é dado pelo espaço nulo de:

$$
\mathrm{M}=\left[\begin{array}{c}
\mathrm{W} \\
\mathbf{X}^{\mathrm{T}}
\end{array}\right]
$$

Se o espaço nulo de $\mathbf{M}$ tem dimensão 2 , então o ponto $\mathbf{X}$ pertence à reta $\mathbf{W}$, caso contrário, $\mathrm{M} \pi=\mathbf{0}$.

O ponto $\mathbf{X}$ definido pela intersecção da reta $W^{*}$ com o plano $\pi$ é dado pelo espaço nulo de:

$$
\mathrm{M}=\left[\begin{array}{l}
\mathrm{W}^{*} \\
\pi^{\mathrm{T}}
\end{array}\right]
$$

Se o espaço nulo de $\mathrm{M}$ tem dimensão 2 , então a reta $\mathrm{W}^{*}$ pertence ao plano $\pi$, caso contrário, $\mathbf{M X}=\mathbf{0}$.

Quádricas e quádricas duais. Uma quádrica é uma superfície em $\mathbf{P}^{3}$ definida pela equação:

$$
\mathbf{X}^{\mathrm{T}} \mathbf{Q} \mathbf{X}=0
$$

onde Q é uma matriz simétrica $4 \times 4$. Muitas das propriedades das quádricas são análogas às propriedades de cônicas: uma quádrica tem 9 graus de liberdade, decorrentes dos 10 elementos independentes de uma matriz simétrica $4 \times 4$ a menos de um fator de escala, e se a matriz Q é singular, a quádrica é degenerada. A intersecção entre um plano $\pi$ e uma quádrica Q é uma cônica C. Dado o sistema de coordenadas definido pela parametrização em (2.19), esta cônica e dada por $\mathrm{C}=\mathrm{M}^{\mathrm{T}} \mathrm{QM}$.

Dada a transformação pontual $\mathbf{X}^{\prime}=\mathrm{HX}$, uma quádrica pontual é transformada por: 


$$
\mathrm{Q}^{\prime}=\mathrm{H}^{-\mathrm{T}} \mathrm{QH}^{-1} \text {. }
$$

A quádrica dual também é uma quádrica. Quádricas duais são equações sobre planos: os planos tangentes à quádrica pontual $Q$ satisfazem $\pi^{\mathrm{T}} \mathrm{Q}^{*} \pi=0$, onde $\mathrm{Q}^{*}$ é a matriz adjunta de $\mathbf{Q}$, ou $\mathbf{Q}^{-1}$ se $\mathbf{Q}$ for inversível. Dada uma transformação $\mathbf{X}^{\prime}=\mathrm{HX}$, a quádrica dual é transformada por:

$$
\mathrm{Q}^{* \prime}=\mathrm{HQ}^{*} \mathrm{H}^{\mathrm{T}} .
$$

Hierarquia de transformações. Analogamente ao caso de transformações planares descritas na Seção 2.1, p.13, as transformações do espaço tridimensional seguem uma hierarquia de subgrupos definidos por sua forma matricial, ou por seus invariantes. A Tabela 2.2 mostra estas transformações, enumerando as propriedades adicionais das transformações do espaço 3D sobre aquelas de suas equivalentes do espaço 2D. Na Tabela $2.2, \mathrm{~A}=\left[a_{i j}\right]$ é uma matriz $3 \times 3$ inversível, $\mathrm{R}=\left[r_{i j}\right]$ é uma matriz de rotação $3 \mathrm{D}, \mathbf{t}=\left(t_{\mathrm{X}}, t_{\mathrm{Y}}, t_{\mathrm{Z}}\right)^{\mathrm{T}}$ é uma translação $3 \mathrm{D}, \mathbf{v}$ é um vetor de três dimensões qualquer, $v$ é um escalar, e $\mathbf{0}=(0,0,0)^{\mathrm{T}}$ é um vetor nulo de três dimensões, e a transformação de uma linha da tabela representa uma generalização da transformação da linha imediatamente inferior, e um caso particular da transformação da linha imediatamente superior, da qual herda todos os invariantes. Os 15 graus de liberdade de uma transformação projetiva dividem-se em 7 para a transformação similar (três para rotação, três para translação, um para escala), 5 para a transformação afim e 3 para a parte projetiva da transformação.

Plano no infinito. Na geometria projetiva planar, a identificação da reta no infinito $\mathbf{l}_{\infty}$ permite determinar as propriedades afins do plano, e a identificação dos pontos circulares sobre $\mathbf{l}_{\infty}$ permite determinar as propriedades métricas. $\mathrm{Na}$ geometria projetiva 3D os equivalentes geométricos destas entidades são respectivamente o plano no infinito $\pi_{\infty}$ e a cônica absoluta $\Omega_{\infty}$.

A posição canônica do plano no infinito é $\pi_{\infty}=(0,0,0,1)^{\mathrm{T}}$. Este plano contém as direções $\mathbf{D}=\left(\mathrm{x}_{1}, \mathrm{x}_{2}, \mathrm{x}_{3}, 0\right)^{\mathrm{T}}$, e permite identificar propriedades afins como o paralelismo. Dois planos são paralelos se e somente se a reta definida por sua intersecção está em $\pi_{\infty}$, e uma reta é paralela a outra reta ou a um plano se o ponto de intersecção está sobre $\pi_{\infty}$.

O plano $\pi_{\infty}$ representa os 3 graus de liberdade necessários para determinar propriedades afins em um sistema de coordenadas projetivo. Estes 3 graus de 
Tabela 2.1: Propriedades geométricas invariantes das transformações 2D.

\begin{tabular}{lc}
\hline \multicolumn{1}{c}{ Grupo } & Matriz \\
\hline Projetiva \\
8GDL
\end{tabular}$\quad\left[\begin{array}{lll}h_{11} & h_{12} & h_{13} \\
h_{21} & h_{22} & h_{23} \\
h_{31} & h_{32} & h_{33}\end{array}\right]$

Concorrência, colinearidade, ordem de contato e razão cruzada (razão entre razões de comprimento).

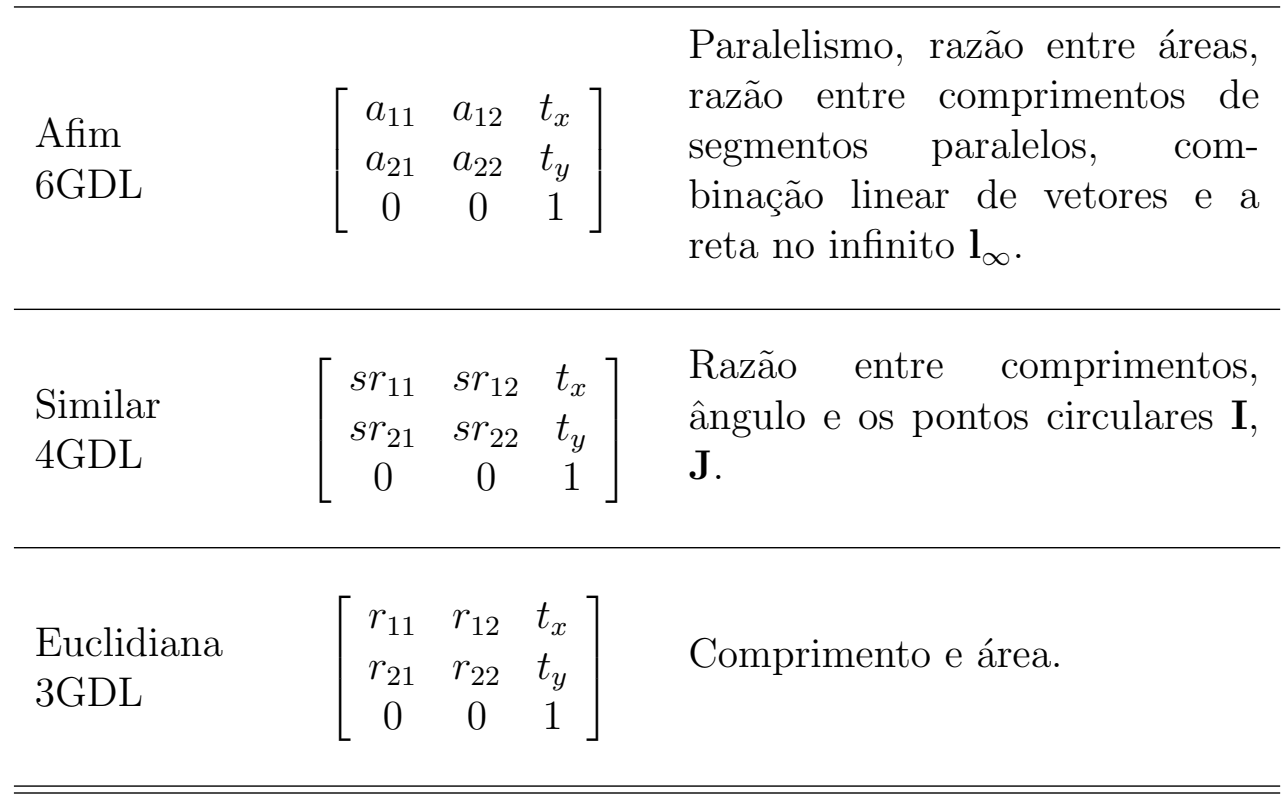

Tabela 2.2: Propriedades geométricas invariantes das transformações 3D.

\begin{tabular}{lll}
\hline \hline \multicolumn{1}{c}{ Grupo } & Matriz & \multicolumn{1}{c}{ Invariantes } \\
\hline $\begin{array}{l}\text { Projetiva } \\
\text { 15GDL }\end{array}$ & {$\left[\begin{array}{cc}\mathbf{A} & \mathbf{t} \\
\mathbf{v}^{\mathrm{T}} & v\end{array}\right]$} & $\begin{array}{l}\text { Intersecção e tangência de } \\
\text { superfícies em contato. }\end{array}$ \\
\hline $\begin{array}{l}\text { Afim } \\
\text { 12GDL }\end{array}$ & {$\left[\begin{array}{cc}\mathbf{A} & \mathbf{t} \\
\mathbf{0}^{\mathrm{T}} & 1\end{array}\right]$} & $\begin{array}{l}\text { Paralelismo de planos, razão } \\
\text { entre volumes, centróides, o } \\
\text { plano no infinito } \pi_{\infty} .\end{array}$ \\
\hline
\end{tabular}

Similar
7 GDL $\quad\left[\begin{array}{cc}s \mathrm{R} & \mathbf{t} \\ \mathbf{0}^{\mathrm{T}} & 1\end{array}\right] \quad$ A cônica absoluta $\Omega_{\infty}$.

Euclidiana $6 \mathrm{GDL}$ $\left[\begin{array}{cc}R & t \\ 0^{\mathrm{T}} & 1\end{array}\right] \quad$ Volume. 
liberdade determinam a componente projetiva de uma homografia em geral com 15 graus de liberdade, comparada à transformação afim - com 12 graus de liberdade.

O plano no infinito $\pi_{\infty}$ é um plano fixo segundo a transformação projetiva $\mathrm{H}$ se e somente se $\mathrm{H}$ é uma transformação afim. O plano $\pi_{\infty}$ em geral é fixo apenas como conjunto segundo a transformação afim, e não fixo ponto a ponto. Segundo uma transformação afim particular, podem haver outros planos fixos além de $\pi_{\infty}$, mas somente $\pi_{\infty}$ é fixo para qualquer transformação afim.

O espaço projetivo $\mathbf{P}^{\mathbf{2}}$ relaciona-se ao espaço projetivo $\mathbf{P}^{\mathbf{3}}$ de forma que um plano $\pi$ cruza o plano $\pi_{\infty}$ na reta no infinito $\mathbf{l}_{\infty}$ do plano $\pi$, e uma transformação projetiva de $\mathbf{P}^{\mathbf{3}}$ induz uma transformação projetiva planar subordinada em $\pi$.

Cônica absoluta. A cônica absoluta $\Omega_{\infty}$ é uma cônica pontual localizada sobre $\pi_{\infty}=(0,0,0,1)^{\mathrm{T}}$, cujos pontos satisfazem:

$$
\left.\begin{array}{r}
\mathrm{x}_{1}^{2}+\mathrm{x}_{2}^{2}+\mathrm{x}_{3}^{2} \\
\mathrm{x}_{4}
\end{array}\right\}=0 .
$$

A forma canônica de $\Omega_{\infty}$ corresponde à cônica $C=I$ no sistema de coordenadas Euclidiano, formada por pontos puramente imaginários em $\pi_{\infty}$.

A cônica $\Omega_{\infty}$ representa os 5 graus de liberdade necessários para determinar propriedades métricas em um sistema de coordenadas afim.

A cônica absoluta $\Omega_{\infty}$ é uma cônica fixa segundo a transformação projetiva $\mathrm{H}$ se e somente se $\mathrm{H}$ é uma similaridade. A cônica $\Omega_{\infty}$ em geral é fixa segundo a transformação similar apenas como conjunto, e não ponto a ponto. Todas circunferências interceptam $\Omega_{\infty}$ em dois pontos. Dado um plano qualquer $\pi$, este cruza $\pi_{\infty}$ em uma reta, que cruza $\Omega_{\infty}$ em dois pontos - os pontos circulares de $\pi$. Todas esferas cruzam $\pi_{\infty}$ em $\Omega_{\infty}$.

Dadas duas retas com direções (vetores de 3 dimensões) $\mathbf{d}_{1}$ e $\mathbf{d}_{2}$, o ângulo entre estas direções no espaço Euclidiano é dado por:

$$
\cos \theta=\frac{\left(\mathbf{d}_{1}{ }^{\mathrm{T}} \mathbf{d}_{2}\right)}{\sqrt{\left(\mathbf{d}_{1}{ }^{\mathrm{T}} \mathbf{d}_{1}\right)\left(\mathbf{d}_{2}{ }^{\mathrm{T}} \mathbf{d}_{2}\right)}} .
$$

Em coordenadas homogêneas de $\mathbf{P}^{\mathbf{3}}$, tem-se:

$$
\cos \theta=\frac{\left(\mathbf{d}_{1}{ }^{\mathrm{T}} \Omega_{\infty} \mathbf{d}_{2}\right)}{\sqrt{\left(\mathbf{d}_{1}{ }^{\mathrm{T}} \Omega_{\infty} \mathbf{d}_{1}\right)\left(\mathbf{d}_{2}{ }^{\mathrm{T}} \Omega_{\infty} \mathbf{d}_{2}\right)}}
$$


onde $\mathbf{d}_{1}$ e $\mathbf{d}_{2}$ agora representam os pontos de intersecção das retas com o plano $\pi_{\infty}$ contendo a cônica $\Omega_{\infty}$. Adicionalmente, Duas direções $\mathbf{d}_{1}$ e $\mathbf{d}_{2}$ são ortogonais se $\mathbf{d}_{1}{ }^{\mathrm{T}} \Omega_{\infty} \mathbf{d}_{2}=0$.

Quádrica dual absoluta. A estrutura dual da cônica absoluta $\Omega_{\infty}$ é uma quádrica degenerada do espaço tridimensional chamada quádrica dual absoluta, denotada $Q_{\infty}^{*}$. Geometricamente, $Q_{\infty}^{*}$ consiste de planos tangentes a $\Omega_{\infty}$. Algebricamente, $Q_{\infty}^{*}$ é representada por uma matriz homogênea $4 \times 4$ de posto 3 , cuja forma canônica no espaço tridimensional Euclidiano é dada por:

$$
\mathrm{Q}_{\infty}^{*}=\left[\begin{array}{cc}
\mathrm{I} & \mathbf{0} \\
\mathbf{0}^{\mathrm{T}} & 0
\end{array}\right]
$$

A quádrica dual absoluta $Q_{\infty}^{*}$ é uma quádrica degenerada e tem 8 graus de liberdade, e representa os graus de liberdade necessários para determinar propriedades métricas em um sistema de coordenadas projetivo. A quádrica dual absoluta $Q_{\infty}^{*}$ é uma forma conveniente para o cálculo algébrico pois encapsula as informações de $\pi_{\infty}$ e de $\Omega_{\infty}$.

A quádrica dual absoluta $\mathrm{Q}_{\infty}^{*}$ é fixa segundo a transformação projetiva $\mathrm{H}$ se e somente se $\mathrm{H}$ é uma similaridade. Adicionalmente, o plano no infinito $\pi_{\infty}$ é o vetor nulo de $\mathrm{Q}_{\infty}^{*}$. O ângulo entre dois planos $\pi_{1}$ e $\pi_{2}$ é dado por:

$$
\cos \theta=\frac{\left(\pi_{1}{ }^{\mathrm{T}} \mathrm{Q}_{\infty}^{*} \pi_{2}\right)}{\sqrt{\left(\mathbf{p i}_{1}{ }^{\mathrm{T}} \mathrm{Q}_{\infty}^{*} \pi_{1}\right)\left(\pi_{2}{ }^{\mathrm{T}} \mathrm{Q}_{\infty}^{*} \pi_{2}\right)}}
$$




\section{Modelo de Câmera}

Uma câmera representa uma relação entre o espaço tridimensional e uma imagem bidimensional. São de especial interesse para este estudo as câmeras de projeção central. Os modelos apresentados a seguir são descritos por matrizes com propriedades específicas que representam o mapeamento realizado pela câmera.

As câmeras que modelam projeção central são casos particulares da câmera projetiva geral, cuja anatomia é examinada através de ferramentas de geometria projetiva, apresentadas no Capítulo 2. As propriedades geométricas da câmera, como seu centro de projeção e o plano de imagem, podem ser extraídas de sua representação matricial de maneira trivial. Casos especiais da câmera projetiva geral herdam suas propriedades, utilizando as mesmas expressões algébricas para cálculo de sua geometria.

Os modelos especializados derivados da câmera projetiva geral são classificados em dois grandes grupos: os modelos de câmeras com centro finito e os modelos de câmera com centro "no infinito". Dentre as câmeras infinitas, a mais importante é a câmera afim, uma generalização da projeção paralela.

Os conceitos apresentados neste capítulo foram extraídos de (HARTLEY; ZISSERMAN, 2000), (FAUGERAS, 1993), (ULLMAN, 1979), e (XU; ZHANG, 1996). A notação adotada e as ilustrações das figuras são de (HARTLEY; ZISSERMAN, 2000).

\subsection{Câmeras Finitas}

O modelo de câmera mais específico e também mais simples é o modelo básico de câmera de orifício, do qual são progressivamente derivados os modelos mais genéricos, em uma série de graduações. Tais modelos são projetados principalmente para descrever o comportamento de câmeras de sensor CCD (do inglês Charge-Coupled Device), mas também podem ser aplicados a outros tipos de câmeras, como imagens de raios-X, negativos fotográficos digitalizados e fotografias ampliadas digitalizadas, entre outros. 

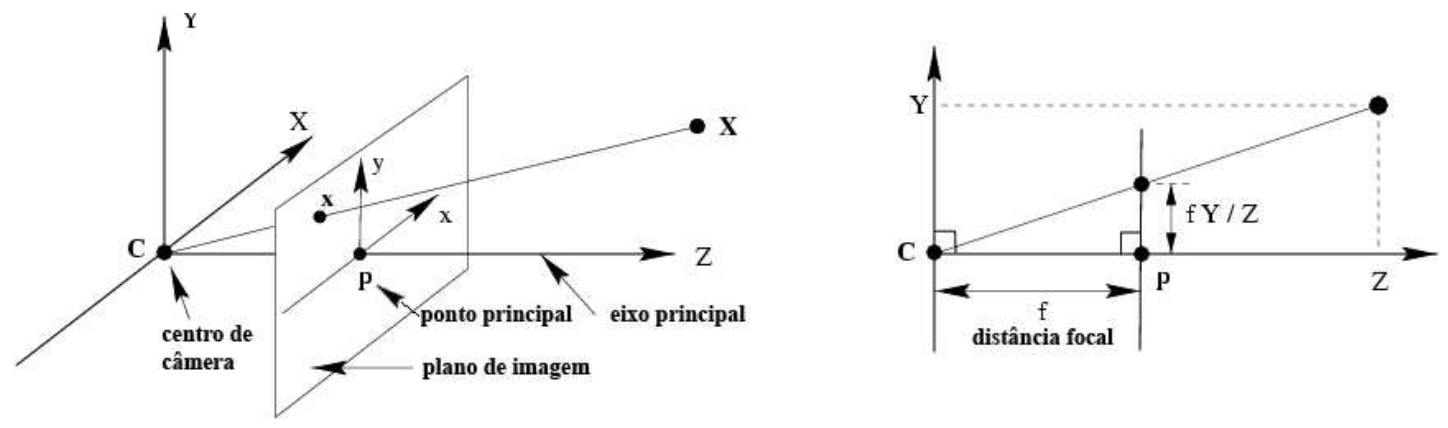

Figura 3.1: Geometria da Câmera de Orifício.

\subsubsection{Modelo Básico de Câmera de Orifício}

Considerando a projeção central de pontos do espaço sobre um plano, seja o centro de projeção a origem de um sistema de coordenadas euclidianas, e seja o plano, chamado plano de imagem ou plano focal.

Segundo o modelo de câmera de orifício, um ponto 3D com coordenadas $\mathbf{X}=(\mathrm{X}, \mathrm{Y}, \mathrm{Z})^{\mathrm{T}}$ é mapeado para o ponto onde a reta que passa por $\mathbf{X}$ e pelo centro de projeção encontra o plano de imagem, conforme mostrado na Figura 3.1. Desta forma, o ponto $(\mathrm{X}, \mathrm{Y}, \mathrm{z})^{\mathrm{T}}$ é mapeado para o ponto $(f \mathrm{X} / \mathrm{Z}, f \mathrm{Y} / \mathrm{Z})^{\mathrm{T}}$ sobre o plano de imagem. Ignorando a coordenada $\mathrm{Z}$, tem-se:

$$
(\mathrm{X}, \mathrm{Y}, \mathrm{Z})^{\mathrm{T}} \mapsto(f \mathrm{X} / \mathrm{Z}, f \mathrm{Y} / \mathrm{Z})^{\mathrm{T}} .
$$

A relação (3.1) descreve o mapeamento da projeção central 3D para coordenadas de imagem. Trata-se de uma relação entre o espaço Euclidiano tridimensional $\mathbf{R}^{\mathbf{3}}$ e o espaço Euclidiano bidimensional $\mathbf{R}^{\mathbf{2}}$.

O centro de projeção é chamado centro de câmera ou centro óptico. A reta perpendicular ao plano de imagem que passa pelo centro de câmera é chamada de eixo principal ou raio principal da câmera, e o ponto onde o eixo principal encontra o plano de imagem é chamado de ponto principal. O plano paralelo ao plano de imagem que contém o centro de câmera é chamado de plano principal da câmera.

\subsubsection{Projeção Central em Coordenadas Homogêneas}

Representando os pontos tridimensionais e os pontos da imagem por vetores de coordenadas homogêneas, pode-se expressar a projeção central como um mapeamento linear entre estes vetores. Desta forma, a relação (3.1) pode ser escrita 


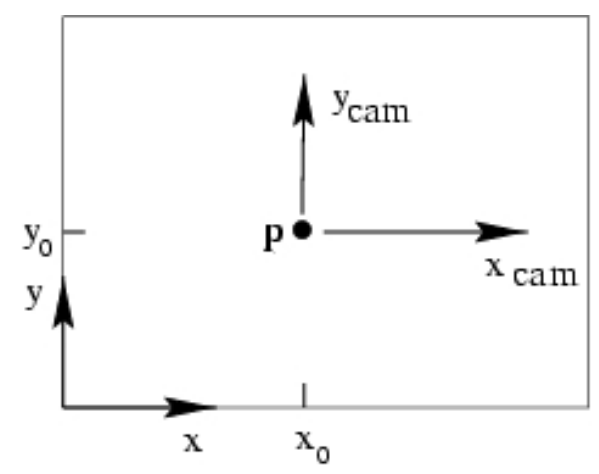

Figura 3.2: Coordenadas de imagem $(x, y)$ e de câmera $\left(x_{\text {cam }}, y_{\text {cam }}\right)$.

como uma multiplicação matricial:

$$
\left(\begin{array}{c}
\mathrm{X} \\
\mathrm{Y} \\
\mathrm{Z} \\
1
\end{array}\right) \mapsto\left(\begin{array}{c}
f \mathrm{X} \\
f \mathrm{Y} \\
\mathrm{Z}
\end{array}\right)=\left[\begin{array}{llll}
f & & & 0 \\
& f & & 0 \\
& & 1 & 0
\end{array}\right]\left(\begin{array}{c}
\mathrm{X} \\
\mathrm{Y} \\
\mathrm{Z} \\
1
\end{array}\right)
$$

A matriz na expressão acima pode ser escrita como $\operatorname{diag}(f, f, 1)[\mathrm{I} \mid \mathbf{0}]$, onde $\operatorname{diag}(f, f, 1)$ é uma matriz diagonal e $[\mathrm{I} \mid \mathbf{0}]$ representa uma matriz dividida em um bloco $3 \times 3$ (matriz identidade) e um vetor-coluna de zeros. Sendo $\mathbf{X}$ o ponto tridimensional representado pelo vetor homogêneo $(\mathrm{X}, \mathrm{Y}, \mathrm{Z}, 1)^{\mathrm{T}}$, e $\mathbf{x}$ o ponto da imagem representado por pelo vetor homogêneo $(f \mathrm{X}, f \mathrm{Y}, \mathrm{Z})^{\mathrm{T}}$, e definindo $\mathrm{P}$ como a matriz homogênea $3 \times 4$, escreve-se (3.2) de forma compacta como $\mathbf{x}=\mathrm{PX}$. Assim, define-se a matriz de projeção da câmera para o modelo de projeção central de câmera de orifício como $\mathrm{P}=\operatorname{diag}(f, f, 1)[\mathrm{I} \mid \mathbf{0}]$.

\subsubsection{Deslocamento do Ponto Principal}

A expressão (3.1) assume que a origem do sistema de coordenadas no plano da imagem está sobre o ponto principal. Na prática, esta condição pode não se aplicar, conforme ilustrado na Figura 3.2. Assim, pode-se generalizar (3.1) como:

$$
(\mathrm{X}, \mathrm{Y}, \mathrm{Z})^{\mathrm{T}} \mapsto\left(f \mathrm{X} / \mathrm{Z}+x_{0}, f \mathrm{Y} / \mathrm{Z}+y_{0}\right)^{\mathrm{T}},
$$

onde o ponto $\left(x_{0}, y_{0}\right)$ representa as coordenadas do ponto principal. Em coordenadas homogêneas tem-se: 


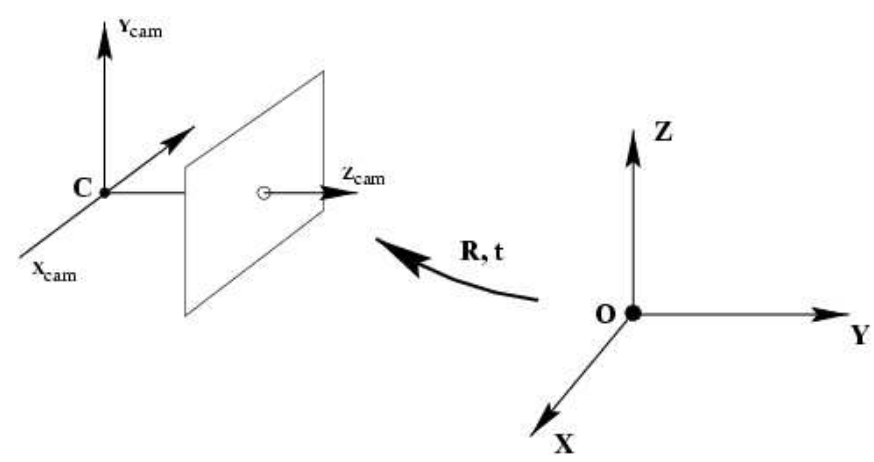

Figura 3.3: Transformação Euclidiana entre os sistemas de coordenadas global e de câmera.

$$
\left(\begin{array}{c}
\mathrm{X} \\
\mathrm{Y} \\
\mathrm{Z} \\
1
\end{array}\right) \mapsto\left(\begin{array}{c}
f \mathrm{X}+x_{0} \\
f \mathrm{Y}+y_{0} \\
\mathrm{Z}
\end{array}\right)=\left[\begin{array}{cccc}
f & & x_{0} & 0 \\
& f & y_{0} & 0 \\
& & 1 & 0
\end{array}\right]\left(\begin{array}{c}
\mathrm{X} \\
\mathrm{Y} \\
\mathrm{Z} \\
1
\end{array}\right)
$$

Chama-se matriz de calibração da câmera a matriz definida por:

$$
\mathrm{K}=\left[\begin{array}{lll}
f & & x_{0} \\
& f & y_{0} \\
& & 1
\end{array}\right]
$$

Assim, a forma resumida de (3.4) é dada por:

$$
\mathbf{x}=\mathrm{K}[\mathrm{I} \mid \mathbf{0}] \mathbf{X}_{\mathrm{cam}},
$$

onde $\mathbf{X}_{\mathrm{cam}}=(\mathrm{X}, \mathrm{Y}, \mathrm{Z}, 1)^{\mathrm{T}}$, indicando que o ponto está representado no sistema de coordenadas Euclidiano cuja origem coincide com o centro de câmera e cujo eixo Z coincide com o eixo principal. Este sistema de coordenadas é chamado de sistema de coordenadas de câmera.

\subsubsection{Rotação e Translação de Câmera}

Em geral, pontos 3D são descritos em relação a um sistema de coordenadas Euclidiano diferente do sistema de coordenadas de câmera, chamado de sistema de coordenadas global. Os dois sistemas de coordenadas estão relacionados por rotação dos eixos e translação da origem, conforme ilustrado na Figura 3.3.

Se $\tilde{\mathbf{X}}$ é um vetor não-homogêneo de três elementos representando as coor- 
denadas de um ponto no sistema de coordenadas global, e $\tilde{\mathbf{X}}_{\text {cam }}$ representa o mesmo ponto no sistema de coordenadas de câmera, então pode-se escrever $\tilde{\mathbf{X}}_{\text {cam }}=\mathrm{R}(\tilde{\mathbf{X}}-\tilde{\mathbf{C}})$, onde $\tilde{\mathbf{C}}$ representa as coordenadas do centro de câmera no sistema de coordenadas global, e R é uma matriz de rotação $3 \times 3$ representando a orientação do sistema de coordenadas de câmera. Utilizando coordenadas homogêneas, tem-se:

$$
\mathbf{X}_{\mathrm{cam}}=\left[\begin{array}{cc}
\mathrm{R} & -\mathrm{R} \tilde{\mathbf{C}} \\
\mathbf{0}^{\mathrm{T}} & 1
\end{array}\right]\left(\begin{array}{l}
\mathrm{X} \\
\mathrm{Y} \\
\mathrm{Z} \\
1
\end{array}\right)=\left[\begin{array}{cc}
\mathrm{R} & -\mathrm{R} \tilde{\mathbf{C}} \\
\mathbf{0}^{\mathrm{T}} & 1
\end{array}\right] \mathbf{X}
$$

Utilizando (3.7) em conjunto com (3.6) tem-se:

$$
\mathbf{x}=\mathrm{KR}[\mathrm{I} \mid-\tilde{\mathbf{C}}] \mathbf{X}
$$

onde o ponto $\mathbf{X}$ é expresso no sistema de coordenadas global. A equação (3.8) representa o mapeamento realizado pela câmera de orifício de forma mais generalizada, expresso matricialmente por:

$$
\mathrm{P}=\mathrm{KR}[\mathrm{I} \mid-\tilde{\mathbf{C}}]
$$

Nota-se que a câmera de orifício geral da equação (3.9) tem 9 graus de liberdade: 3 provenientes de $\mathrm{K}$ (os elementos $f, x_{0}$ e $y_{0}$ ), 3 de $\mathrm{R}$, e 3 de $\tilde{\mathbf{C}}$. Os parâmetros provenientes de $\mathrm{K}$ são chamados de parâmetros intrínsecos, ou o-rientação interna, da câmera. Os parâmetros provenientes de $\mathrm{R}$ e $\tilde{\mathbf{C}}$, que relacionam a posição e a orientação da câmera no sistema de coordenadas global, são chamados de parâmetros extrínsecos, ou orientação externa, da câmera. Em geral, é conveniente ocultar a referência ao centro de câmera e representar a transformação de coordenadas globais para coordenadas de câmera por $\tilde{\mathbf{X}}_{\text {cam }}=\mathrm{R} \tilde{\mathbf{X}}+\mathbf{t}$, onde $\mathbf{t}=-\mathrm{R} \tilde{\mathbf{C}}$. Neste caso a matriz de câmera é dada por:

$$
\mathrm{P}=\mathrm{K}[\mathrm{R} \mid \mathbf{t}]
$$

\subsubsection{Câmeras CCD}

O modelo de câmera de orifício apresentado anteriormente supõe que as coordenadas de imagem sejam Euclidianas e possuam escalas idênticas em ambos os 
eixos de direção. No caso de câmeras CCD, é possível que os pixel não sejam quadrados. Se as coordenadas de imagem são medidas em pixel, introduzem-se fatores de escala diferentes em cada direção. Se o número de pixel por unidade de distância em coordenadas de imagem é $m_{x}$ na direção X, e $m_{y}$ na direção Y, então obtém-se a transformação para coordenadas de pixel multiplicando-se (3.5) à esquerda por $\operatorname{diag}\left(m_{x}, m_{y}, 1\right)$. Assim, a forma geral da matriz de calibração para câmeras CCD é dada por:

$$
\mathrm{K}=\left[\begin{array}{ccc}
\alpha_{x} & & x_{0}^{\prime} \\
& \alpha_{x} & y_{0}^{\prime} \\
& & 1
\end{array}\right]
$$

Os parâmetros $\alpha_{x}=f m_{x}$ e $\alpha_{y}=f m_{y}$ representam a distância focal da câmera em função das dimensões de pixel nas direções X e Y respectivamente. Analogamente, $\mathbf{p}^{\prime}=\left(x_{0}^{\prime}, y_{0}^{\prime}\right)^{\mathrm{T}}$ é o ponto principal em termos de dimensão de pixel, com coordenadas $x_{0}^{\prime}=m_{x} x_{0}$ e $y_{0}^{\prime}=m_{y} y_{0}$. Desta forma, uma câmera CCD tem 10 graus de liberdade.

\subsubsection{Câmera Projetiva Finita}

Com o intuito de adicionar generalidade, pode-se considerar a matriz de calibração geral como:

$$
\mathrm{K}=\left[\begin{array}{ccc}
\alpha_{x} & s & x_{0}^{\prime} \\
& \alpha_{x} & y_{0}^{\prime} \\
& & 1
\end{array}\right],
$$

onde o parâmetro adicional $s$ é chamado de parâmetro de inclinação, que será diferente de zero quando os eixos X e Y não forem ortogonais. Este parâmetro será nulo para a maioria das câmeras, mas pode assumir valores diferentes de zero em situações especiais, como na imagem de uma imagem, ou na ampliação de um negativo quando o eixo principal da lente de ampliação não é perpendicular ao plano de imagem original.

Uma câmera $\mathrm{P}=\mathrm{KR}[\mathrm{I} \mid-\tilde{\mathbf{C}}]$ com a matriz $\mathrm{K}$ conforme (3.12) é chamada de câmera projetiva finita, e possui 11 graus de liberdade - o mesmo que uma matriz $3 \times 4$ menos de um fator de escala arbitrário. Nota-se que a sub-matriz $3 \times 3$ da esquerda de $\mathrm{P}$ é não-singular, e que o conjunto de matrizes de câmera referentes a câmeras projetivas finitas é idêntico ao conjunto de matrizes homogêneas $3 \times 4$ 
para as quais a sub-matriz $3 \times 3$ da esquerda é não-singular.

\subsubsection{Câmera Projetiva Geral}

O último passo na hierarquia de câmeras projetivas é a remoção da restrição de não-singularidade da sub-matriz $3 \times 3$ da esquerda da matriz de câmera, que garante que esta representa uma câmera projetiva finita. Uma câmera projetiva geral é aquela representada por uma matriz homogênea $3 \times 4$ arbitrária de posto 3, e tem 11 graus de liberdade. S o posto da matriz for menor que 3 então o espaço de mapeamento da matriz será uma reta ou um ponto, e não um plano completo (o mapeamento não será bidimensional).

\subsection{Anatomia da Câmera Projetiva}

Uma câmera projetiva pode ser decomposta em blocos, de acordo com $\mathrm{P}=\left[\mathrm{M} \mid \mathbf{p}_{4}\right]$, onde M é uma matriz $3 \times 3$. Se M for não-singular, trata-se de uma câmera finita, caso contrário é uma câmera infinita.

Centro de câmera. A matriz $\mathrm{P}$ tem espaço nulo à direita unidimensional, pois tem posto 3 e possui 4 colunas. Supondo que o espaço nulo seja gerado pelo vetor $\mathbf{C}$, então $\mathbf{P C}=\mathbf{0}$. Assim, $\mathbf{C}$ é o centro de câmera, representado por um vetor homogêneo de 4 elementos. Considerando a reta contendo $\mathbf{C}$ e outro ponto 3D qualquer A, pontos sobre esta reta podem ser representados pela relação:

$$
\mathbf{X}(\lambda)=\lambda \mathbf{A}+(1-\lambda) \mathbf{C}, \lambda \in \mathbf{R} .
$$

Segundo a relação $\mathbf{x}=\mathbf{P X}$, e como $\mathbf{P C}=\mathbf{0}$, os pontos sobre esta reta são projetados para:

$$
\mathbf{x}=\mathrm{PX}(\lambda)=\lambda \mathbf{P A}+(1-\lambda) \mathrm{P} \mathbf{C}=\lambda \mathbf{P A} .
$$

Assim, todos pontos sobre a reta são mapeados para o mesmo ponto da imagem, PA, significando que a reta é raio passando pelo centro de câmera. Conseqüentemente, $\mathbf{C}$ é a representação homogênea do centro de câmera, pois qualquer que seja a escolha de $\mathbf{A}$, a reta $\mathbf{X}(\lambda)$ é um raio passando pelo centro de câmera.

Observa-se que o ponto da imagem $\mathbf{P C}=(0,0,0)^{\mathrm{T}}$ é indefinido, e o centro de câmera é o único ponto do espaço para o qual a imagem é indefinida. No caso de 

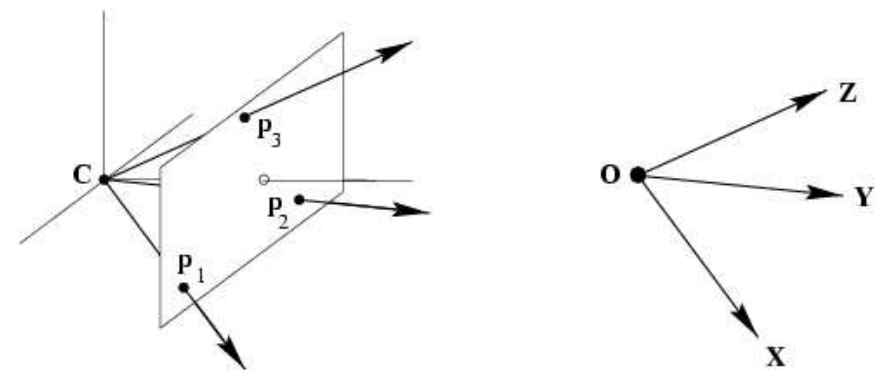

Figura 3.4: Vetores coluna da matriz de câmera.

câmeras finitas, o resultado pode ser estabelecido diretamente, pois $\mathbf{C}=\left(\tilde{\mathbf{C}}^{\mathrm{T}}, 1\right)^{\mathrm{T}}$ é o vetor-nulo de $\mathrm{P}=\mathrm{KR}[\mathrm{I} \mid-\tilde{\mathbf{C}}]$. Este resultado é válido mesmo quando M é singular. Neste caso, o vetor-nulo tem a forma $\mathbf{C}=\left(\mathbf{d}^{\mathrm{T}}, 0\right)^{\mathrm{T}}$, onde $\mathbf{M d}=0$, e o centro de câmera será um ponto no infinito.

Vetores Coluna. As colunas da câmera projetiva são vetores de 3 elementos que possuem significado geométrico como pontos especiais da imagem. Utilizando a notação em que as colunas de $\mathrm{P}$ são $\mathbf{p}_{i} i=1, \ldots, 4$, então $\mathbf{p}_{1}, \mathbf{p}_{2}$ e $\mathbf{p}_{3}$ são respectivamente os pontos de fuga dos eixos de coordenadas $\mathrm{X}$, Y e Z do sistema de coordenadas global, como ilustrado na Figura 3.4. A coluna $\mathbf{p}_{4}$ é a imagem da origem do sistema de coordenadas global.

Vetores Linha. As linhas da matriz da câmera projetiva definida em (3.15) são vetores de 4 elementos cujas interpretações geométricas são planos do espaço $3 \mathrm{D}$. Denominado as linhas de $\mathrm{P}$ por $\mathbf{p}^{i^{\mathrm{T}}}, i=1, \ldots, 3$, tem-se:

$$
\mathrm{P}=\left[\begin{array}{llll}
p_{11} & p_{12} & p_{13} & p_{14} \\
p_{21} & p_{22} & p_{23} & p_{24} \\
p_{31} & p_{32} & p_{33} & p_{34}
\end{array}\right]=\left[\begin{array}{c}
\mathbf{p}^{1^{\mathrm{T}}} \\
\mathbf{p}^{2^{\mathrm{T}}} \\
\mathbf{p}^{3^{\mathrm{T}}}
\end{array}\right]
$$

Plano Principal. O plano principal é o plano contendo o centro de câmera paralelo ao plano da imagem. Consiste do conjunto de pontos $\mathbf{X}$ que são projetados sobre a reta no infinito da imagem - ou seja, $\mathbf{P X}=(x, y, 0)^{\mathrm{T}}$. Assim, um ponto está sobre o plano principal se e somente se $\mathbf{p}^{3^{\mathrm{T}}} \mathbf{X}=0$. Desta forma, $\mathbf{p}^{3}$ é o vetor que representa o plano principal da câmera. Se $\mathbf{C}$ é o centro de câmera, então $\mathbf{P C}=\mathbf{0}$, e em particular $\mathbf{p}^{3^{\mathrm{T}}} \mathbf{C}=0$, pois $\mathbf{C}$ pertence ao plano principal da câmera.

Planos dos Eixos. Considerando o conjunto de pontos $\mathbf{X}$ pertencentes ao plano $\mathbf{p}^{1}$, estes pontos satisfazem a relação $\mathbf{p}^{1^{\mathrm{T}}} \mathbf{X}=0$, e são projetados em $\mathbf{P X}=(0, y, w)^{\mathrm{T}}-$ são pontos do eixo $\mathrm{Y}$ da imagem. Como $\mathbf{P C}=\mathbf{0}$, temos 

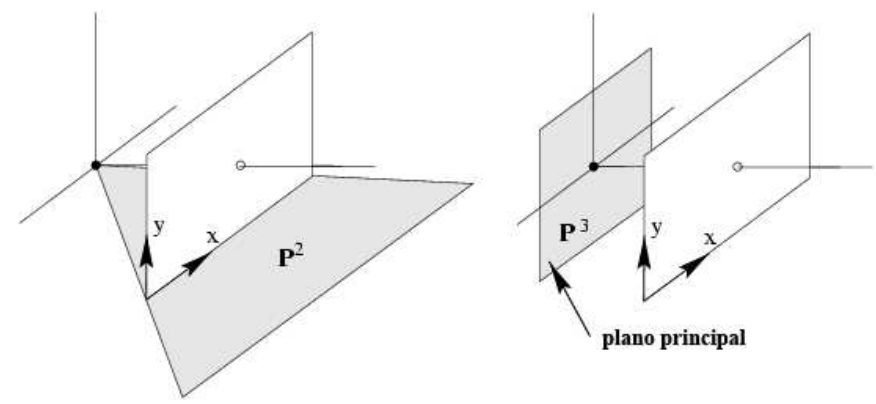

Figura 3.5: Planos $\mathbf{p}^{2^{\mathrm{T}}}$ e $\mathbf{p}^{3^{\mathrm{T}}}$ definidos pelas linhas da matriz de câmera.

$\mathbf{p}^{1^{\mathrm{T}}} \mathbf{C}=0$, e $\mathbf{C}$ também está sobre o plano $\mathbf{p}^{1}$. Assim o plano $\mathbf{p}^{1}$ é definido pelo centro de câmera e pela reta $x=0$ da imagem. Analogamente, o plano $\mathbf{p}^{2}$ é definido pelo centro de câmera e a reta $y=0$ da imagem.

Ao contrário do plano principal $\mathbf{p}^{3}$, os planos de eixos $\mathbf{p}^{1}$ e $\mathbf{p}^{2}$ dependem dos eixos X e Y da imagem - dependem da escolha do sistema de coordenadas da imagem, e estão menos acoplados à geometria da câmera do que o plano principal. Em particular, a reta de intersecção dos planos $\mathbf{p}^{1}$ e $\mathbf{p}^{2}$ é a reta que passa pelo centro de câmera e pela origem do sistema de coordenadas da imagem. Esta reta é portanto a projeção inversa da origem do sistema de coordenadas da imagem, que em geral não coincide com o eixo principal da câmera. A Figura 3.5 ilustra estes planos obtidos a partir dos vetores linha de P.

O centro de câmera $\mathbf{C}$ está na intersecção dos três planos $\mathbf{p}^{1^{\mathrm{T}}}, \mathbf{p}^{2^{\mathrm{T}}}$ e $\mathbf{p}^{3^{\mathrm{T}}}$, os quais são sempre distintos pelo fato da matriz de projeção ter posto 3. Algebricamente esta condição é representada por $\mathbf{P C}=\mathbf{0}$, a equação original do centro de câmera.

Ponto Principal. O eixo principal é a reta passando pelo centro de câmera $\mathbf{C}$ perpendicular ao plano principal $\mathbf{p}^{3}$. O eixo principal cruza o plano da imagem no ponto principal. A normal ao plano $\pi=\left(\pi_{1}, \pi_{2}, \pi_{3}, \pi_{4}\right)^{\mathrm{T}}$ é a direção $\left(\pi_{1}, \pi_{2}, \pi_{3}\right)^{\mathrm{T}}$, representada pelo ponto $\left(\pi_{1}, \pi_{2}, \pi_{3}, 0\right)^{\mathrm{T}}$ sobre o plano no infinito. No caso do plano principal da câmera, este ponto é $\hat{\mathbf{p}}^{3}=\left(p_{31}, p_{32}, p_{33}, 0\right)^{\mathrm{T}}$. A projeção deste ponto utilizando a matriz da câmera $\mathrm{P}$ fornece o ponto principal $\mathbf{p}=\mathrm{P} \hat{\mathbf{p}}^{3}$. Somente a sub-matriz $3 \times 3$ à esquerda de $\mathrm{P}=\left[\mathrm{M} \mid \mathbf{p}_{4}\right]$ tem efeito nesta equação, e o ponto principal pode ser calculado por $\mathbf{p}=\mathbf{M m}^{3}$, onde $\mathbf{m}^{3}$ é a terceira linha de $\mathbf{M}$.

Vetor do Eixo Principal. Apesar de qualquer ponto $\mathbf{X}$ fora do plano principal ser mapeado para um ponto da imagem de acordo com $\mathbf{x}=\mathrm{PX}$, na prática somente metade dos pontos do espaço - aqueles em frente à câmera podem ser observados em uma imagem. Dada a matriz de câmera $\mathrm{P}=\left[\mathrm{M} \mid \mathbf{p}_{4}\right]$, 
o vetor $\mathbf{m}^{3}$ definido anteriormente indica a direção do eixo principal. Podese definir este vetor de forma a indicar também o sentido em frente à câmera (sentido positivo). Como P é definida a menos de um fator de escala, incluindo sinal, existe uma ambigüidade sobre se $\mathbf{m}^{3}$ ou $-\mathbf{m}^{3}$ aponta o sentido positivo do eixo principal.

Considerando o sistema de coordenadas de câmera, de acordo com (3.6) a equação de projeção de um ponto tridimensional para um ponto na imagem é dada por $\mathbf{x}=\mathrm{P}_{\text {cam }} \mathbf{X}_{\text {cam }}=\mathrm{K}[\mathrm{I} \mid \mathbf{0}] \mathbf{X}_{\text {cam }}$, onde $\mathbf{X}_{\text {cam é o ponto tridimensional expresso }}$ em coordenadas de câmera. O vetor $v=\operatorname{det}(\mathrm{M}) \mathbf{m}^{3}=(0,0,1)^{\mathrm{T}}$ indica o sentido em frente à câmera, na direção do eixo principal, independente da escala de $\mathrm{P}_{\text {cam }}$. Se o ponto for expresso em coordenadas globais, então $\mathrm{P}=k \mathrm{~K}[\mathrm{R} \mid-\mathrm{R} \tilde{\mathbf{C}}]=\left[\mathrm{M} \mid \mathbf{p}_{4}\right]$, com $\mathrm{M}=k \mathrm{KR}$. Como $\operatorname{det}(\mathrm{R})>0$, novamente o vetor $v=\operatorname{det}(\mathrm{M}) \mathbf{m}^{3}$ não é afetado pela escala. Resumindo, $v=\operatorname{det}(\mathrm{M}) \mathbf{m}^{3}$ é um vetor na direção do eixo principal, no sentido em frente à câmera.

Projeção Inversa de Pontos da Imagem. Dado o ponto $\mathbf{x}$ de uma imagem, existe um conjunto de pontos do espaço que são mapeados para esse ponto. Este conjunto constitui um raio no espaço que passa pelo centro de câmera. Dois pontos sobre o raio são conhecidos: o centro de câmera $\mathbf{C}$ (onde $\mathbf{P C}=\mathbf{0}$ ) e o ponto $\mathrm{P}^{+} \mathbf{x}$, onde $\mathrm{P}^{+}$é a pseudo-inversa ${ }^{1}$ de $\mathrm{P}$. Assim, o raio dos pontos do espaço 3D que são mapeados no ponto $\mathbf{x}$ da imagem é dado por:

$$
\mathbf{X}(\lambda)=\mathrm{P}^{+} \mathbf{x}+\lambda \mathbf{C}
$$

No caso de câmeras finitas, o centro de câmera é dado por $\tilde{\mathbf{C}}=-\mathbf{M}^{-1} \mathbf{p}_{4}$, e um ponto da imagem $\mathbf{x}$ projeta-se inversamente em um raio que cruza o plano no infinito em $\mathbf{D}=\left(\left(\mathrm{M}^{-1} \mathbf{x}\right)^{\mathrm{T}}, 0\right)^{\mathrm{T}}$, e $\mathbf{D}$ fornece o segundo ponto sobre o raio. A reta que passa por estes dois pontos é dada por:

$$
\mathbf{X}(\mu)=\mu\left(\begin{array}{c}
\mathrm{M}^{-1} \mathbf{X} \\
0
\end{array}\right)+\left(\begin{array}{c}
-\mathrm{M}^{-1} \mathbf{p}_{4} \\
1
\end{array}\right)=\left(\begin{array}{c}
\mathrm{M}^{-1}\left(\mu \mathbf{X}-\mathbf{p}_{4}\right) \\
1
\end{array}\right)
$$

Profundidade de Pontos. Dados o ponto tridimensional $\mathrm{X}=(\mathrm{X}, \mathrm{Y}, \mathrm{Z}, \mathrm{T})^{\mathrm{T}}$ e a matriz de câmera de uma câmera finita $\mathrm{P}=\left[\mathrm{M} \mid \mathbf{p}_{4}\right]$, se $\mathbf{P X}=w(x, y, 1)^{\mathrm{T}}$ então a profundidade do ponto $\mathbf{X}$ em frente do plano principal da câmera é dada por:

\footnotetext{
${ }^{1} \mathrm{~A}$ pseudo-inversa de $\mathrm{P}$ é a matriz $\mathrm{P}^{+}$, para a qual $\mathrm{PP}^{+}=\mathrm{I}$.
} 


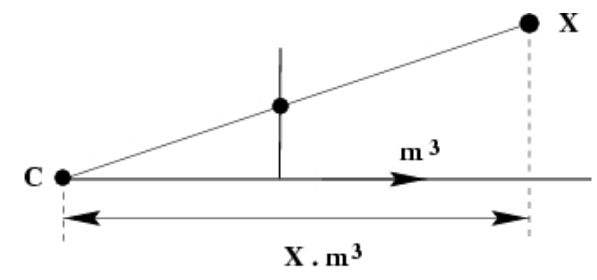

Figura 3.6: Profundidade do ponto $\mathbf{X}$.

$$
\operatorname{depth}(\mathbf{X} ; \mathbf{P})=\frac{\operatorname{sign}(\operatorname{det}(\mathbf{M}) w)}{\mathrm{T}\left\|\mathbf{m}^{3}\right\|}
$$

Considerando $\mathbf{C}=\left(\tilde{\mathbf{C}}^{\mathrm{T}}, 1\right)^{\mathrm{T}}$ o centro de câmera, e o ponto $3 \mathrm{D} \mathbf{X}=\left(\tilde{\mathbf{X}}^{\mathrm{T}}, 1\right)^{\mathrm{T}}$ projetado em um ponto de imagem $\mathbf{x}=w(x, y, 1)^{\mathrm{T}}$, temos $w=\mathbf{p}^{3^{\mathrm{T}}} \mathbf{X}=\mathbf{p}^{3^{\mathrm{T}}}(\mathbf{X}-$ $\mathbf{C})=\mathbf{m}^{3^{\mathrm{T}}}(\tilde{\mathbf{X}}-\tilde{\mathbf{C}})$. Como $\mathbf{m}^{3}$ é a direção do eixo principal, o fator $w$ pode ser interpretado como o produto escalar do raio partindo do centro de câmera e passando por $\mathbf{X}$ com a direção do eixo principal. Se a matriz de câmera for normalizada de forma que $\operatorname{det}(\mathrm{M})>0$ e $\left\|\mathbf{m}^{3}\right\|=1$, então $\mathbf{m}^{3}$ é um vetor unitário no sentido positivo do eixo. Então $w$ pode ser interpretado como a profundidade do ponto $\mathbf{X}$ em relação ao centro de câmera $\mathbf{C}$, na direção do eixo principal, conforme ilustrado na Figura 3.6.

A equação (3.18) é uma forma efetiva de determinar se um ponto $\mathbf{X}$ está em frente à câmera. Verifica-se que o valor de $\operatorname{depth}(\mathbf{X} ; \mathrm{P})$ não se altera ao se multiplicar o ponto $\mathbf{X}$ ou a matriz de câmera $\mathrm{P}$ por um fator constante $k$. Assim, $\operatorname{depth}(\mathbf{X} ; \mathbf{P})$ é independente da representação homogênea particular de $\mathbf{X}$ e P.

\subsection{Lentes: Distorção Radial}

Os modelos de câmera desenvolvidos nas seções anteriores assumem que o modelo linear descreve precisamente o processo de geração de imagens. Desta forma, o ponto 3D e o centro óptico são colineares, retas 3D correspondem a retas da imagem, e assim por diante. No entanto, considerando-se lentes reais, e não câmeras de orifício, tais condições deixam de ser verdadeiras. O efeito mais importante neste caso geralmente é a distorção radial. Na prática, este erro tornase mais significativo a medida que a distância focal da lente diminui, conforme ilustrado na Figura 3.7 - distância focal curta (a) × longa (b). Na Figura 3.7(a) o efeito de distorção radial é evidente nas linhas curvas da periferia da imagem, que correspondem a retas na cena tridimensional.

A distorção radial pode ser eliminada corrigindo-se a imagem para que cor- 


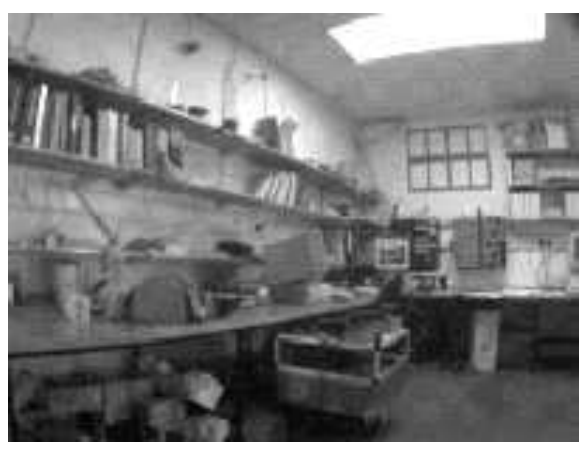

(a)

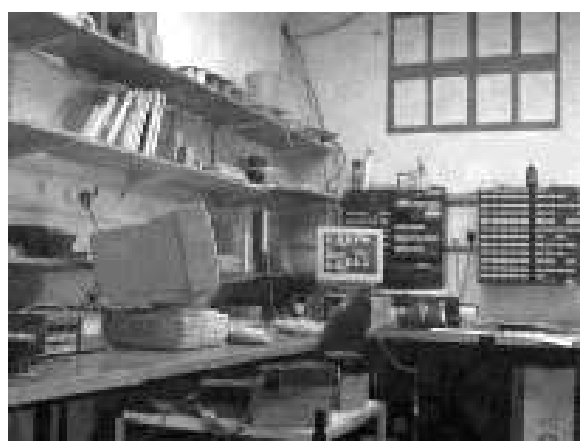

(b)

Figura 3.7: Distorção Radial × Distância Focal. (a) Distância focal curta. (b) Distância focal longa.
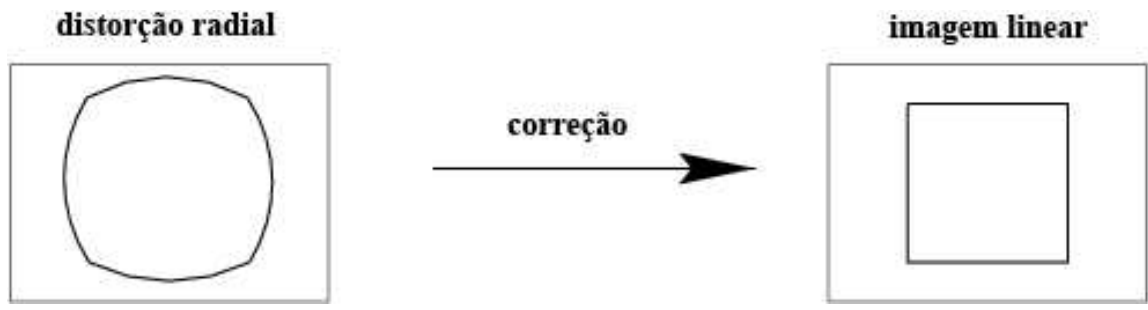

Figura 3.8: Correção da distorção radial.

responda àquela obtida através de uma câmera perfeitamente linear, conforme ilustrado na Figura 3.8, que mostra a correção da imagem de um quadrado apresentando distorção radial significativa para aquela que seria obtida através de uma lente perfeitamente linear. A correção deve ser aplicada na etapa correta do processo de projeção: a distorção devido à lente ocorre durante a projeção 3D para o plano de imagem, de acordo com a equação (3.2). Em seguida, a matriz de calibração (3.12) reflete a escolha de coordenadas da imagem, relacionando as posições no plano de imagem com as coordenadas de pixel.

Sejam $(\tilde{x}, \tilde{y})^{\mathrm{T}}$ as coordenadas de imagem de um ponto 3D $\mathbf{X}$ sob uma câmera de orifício ideal (sem distorção), medidas em múltiplos da distância focal, e $\mathbf{X}_{\text {cam }}$ o ponto 3D expresso em coordenadas de câmera, relacionadas às coordenadas globais por (3.7). Pela equação (3.6) tem-se $(\tilde{x}, \tilde{y}, 1)^{\mathrm{T}}=[\mathrm{I} \mid \mathbf{0}] \mathbf{X}_{\text {cam. }}$. O ponto verdadeiramente projetado corresponde ao deslocamento radial do ponto linear, de forma que a distorção radial (ou distorção de lente) pode ser modelada por:

$$
\left(\begin{array}{l}
x_{d} \\
y_{d}
\end{array}\right)=\mathcal{L}(\tilde{r})\left(\begin{array}{l}
\tilde{x} \\
\tilde{y}
\end{array}\right),
$$

onde o ponto $(\tilde{x}, \tilde{y})^{\mathrm{T}}$ é a posição ideal na imagem (segundo projeção linear), e o 
ponto $\left(x_{d}, y_{d}\right)^{\mathrm{T}}$ é a posição real na imagem, com distorção radial. A função $\mathcal{L}(\tilde{r})$ fornece o fator de distorção em função do raio $\tilde{r}$ (distância radial em relação ao centro: $\left.\sqrt{x_{d}^{2}+y_{d}^{2}}\right)$.

Correção da Distorção. Em coordenadas de pixel, pode-se escrever a correção da distorção radial como:

$$
\hat{x}=x_{c}+\mathcal{L}(r)\left(x-x_{c}\right) \quad \hat{y}=y_{c}+\mathcal{L}(r)\left(y-y_{c}\right),
$$

onde $(x, y)^{\mathrm{T}}$ são as coordenadas reais do ponto na imagem, $(\hat{x}, \hat{y})^{\mathrm{T}}$ são as coordenadas corrigidas, $\left(x_{c}, y_{c}\right)^{\mathrm{T}}$ são as coordenadas do centro da distorção radial, e $r=\sqrt{\left(x-x_{c}\right)^{2}+\left(y-y_{c}\right)^{2}}$. Se a relação de aspecto não for unitária, é necessário corrigir seu efeito no cálculo de $r$. Após esta correção, a relação entre as coordenadas $(\hat{x}, \hat{y})^{\mathrm{T}}$ e as coordenadas do ponto 3D correspondente será a de uma câmera projetiva linear.

Escolha da Função e do Centro de Distorção. A função $\mathcal{L}(r)$ só está definida para valores positivos de $r$, sendo que $\mathcal{L}(0)=1$. Aproxima-se uma função arbitrária $\mathcal{L}(r)$ por uma expansão de Taylor $\mathcal{L}(r) \approx 1+k_{1} r+k_{2} r^{2}+k_{3} r^{3}+\ldots$ Os coeficientes para correção radial $\left\{k_{1}, k_{2}, k_{3}, \ldots, x_{c}, y_{c}\right\}$ são considerados como parte da calibração interna da câmera. Geralmente utiliza-se o ponto principal como centro da distorção radial, ainda que não seja necessário que coincidam exatamente. Tal correção, em conjunto com a matriz de calibração, especifica o mapeamento de um ponto da imagem para um raio no sistema de coordenadas de câmera.

\subsection{Centro de Câmera}

Um objeto 3D e o centro de câmera definem um feixe de raios, e uma imagem é obtida a partir da interseç̧ão destes raios com um plano. Este feixe de raios é chamado de cone de raios, apesar de normalmente não se referir ao cone clássico. Supondo que este cone seja interceptado por dois planos, como mostrado na Figura 3.9, obtém-se duas imagens $\mathcal{I}$ e $\mathcal{I}^{\prime}$. Um raio passando por um ponto 3D $\mathbf{X}$ e pelo centro de câmera $\mathrm{C}$ fura os planos nos pontos $\mathbf{x}$ e $\mathbf{x}^{\prime}$ das imagens. Tais pontos são relacionados através de uma homografia planar $\mathbf{x}^{\prime}=\mathrm{Hx}$. As duas imagens estão relacionadas por um mapa de perspectiva. Isto implica que imagens obtidas a partir de um mesmo centro de câmera podem ser mapeadas entre si através de uma transformação projetiva planar. Em outras palavras, elas são projetivamente equivalentes e possuem as mesmas propriedades projetivas. Uma 


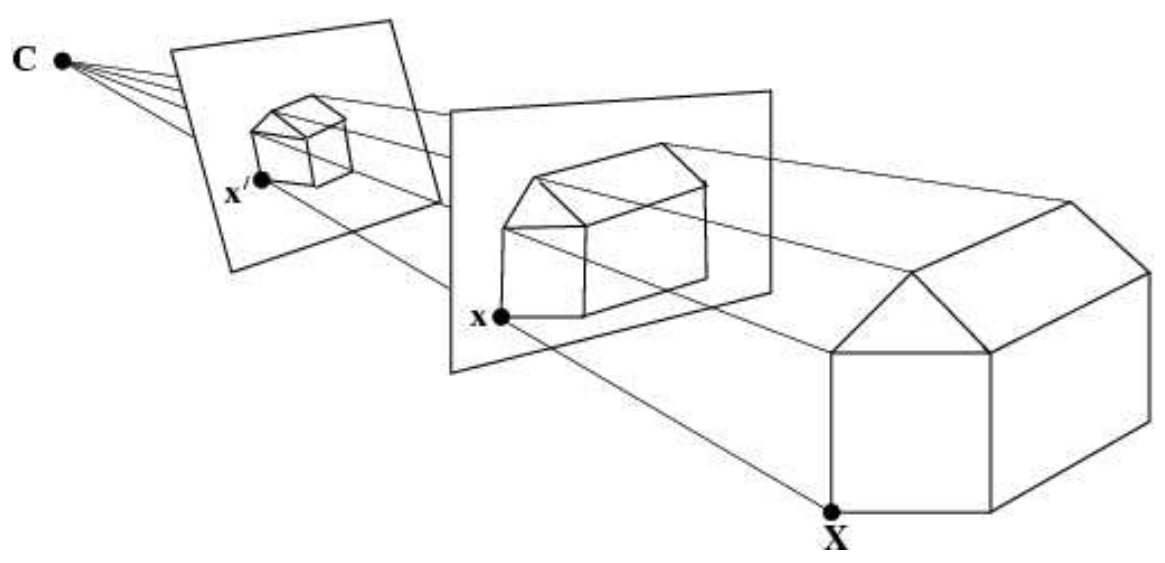

Figura 3.9: Cone de raios com vértice no centro de câmera.

câmera pode, portanto, ser considerada como uma dispositivo de visualização projetivo - determinando propriedades projetivas do cone de raios com vértice no centro de câmera.

Considerando duas câmeras de mesmo centro, $\mathrm{P}=\mathrm{KR}[\mathrm{I} \mid-\tilde{\mathbf{C}}]$ e $\mathrm{P}^{\prime}=\mathrm{K}^{\prime} \mathrm{R}^{\prime}[\mathrm{I} \mid-$ $\tilde{\mathbf{C}}$ ], existe uma relação entre as mesmas, $\mathrm{P}^{\prime}=\mathrm{K}^{\prime} \mathrm{R}^{\prime}(\mathrm{KR})^{-1} \mathrm{P}$, da qual segue que as imagens de um ponto 3D $\mathbf{X}$ dadas pelas duas câmeras são relacionadas por:

$$
\mathbf{x}^{\prime}=\mathrm{P}^{\prime} \mathbf{X}=\left(\mathrm{K}^{\prime} \mathrm{R}^{\prime}\right)(\mathrm{KR})^{-1} \mathbf{x}
$$

$\mathrm{Ou}$ seja, os pontos correspondentes das duas imagens são relacionados por uma homografia planar (uma matriz $3 \times 3$ ), tal que $\mathbf{x}^{\prime}=\mathrm{Hx}$, onde $\mathrm{H}=\left(\mathrm{K}^{\prime} \mathrm{R}^{\prime}\right)(\mathrm{KR})^{-1}$.

\subsubsection{Deslocamento do Plano de Imagem}

Por simplicidade, escolhe-se o sistema de coordenadas global coincidente com o sistema de coordenadas de câmera, de forma que $\mathrm{P}=\mathrm{K}[\mathrm{I} \mid \mathbf{0}]$. Assume-se também que o plano de imagem não contém o centro de câmera, de forma a evitar imagens degeneradas.

Considerando inicialmente um aumento da distância focal, correspondente a um deslocamento do plano de imagem ao longo do eixo principal, o efeito na imagem é de uma simples ampliação. Algebricamente, se $\mathbf{x}$ e $\mathbf{x}^{\prime}$ são imagens do ponto $\mathbf{X}$ antes e depois do zoom, então:

$$
\mathbf{x}=\mathrm{K}[\mathrm{I} \mid \mathbf{0}] \mathbf{X} \quad \text { e } \quad \mathbf{x}^{\prime}=\mathrm{K}^{\prime}[\mathrm{I} \mid \mathbf{0}] \mathbf{X}=\mathrm{K}^{\prime} \mathrm{K}^{-1} \mathrm{~K}[\mathrm{I} \mid \mathbf{0}] \mathbf{X}=\mathrm{K}^{\prime} \mathrm{K}^{-1} \mathbf{X} .
$$

Desta forma, $\mathbf{x}^{\prime}=\mathrm{Hx}$ com $\mathrm{H}=\mathrm{K}^{\prime} \mathrm{K}^{-1}$. Se apenas as distâncias focais diferem 
entre $\mathrm{K}$ e $\mathrm{K}^{\prime}$, então:

$$
\mathrm{K}^{\prime} \mathrm{K}^{-1}=\left[\begin{array}{cc}
k \mathrm{I} & (1-k) \tilde{\mathbf{p}}_{0} \\
\mathbf{0}^{\mathrm{T}} & 1
\end{array}\right]
$$

onde $\tilde{\mathbf{p}}_{0}$ é o ponto principal expresso em coordenadas de imagem, e $k=f^{\prime} / f$ é o fator de ampliação. Utilizando a forma geral da matriz de calibração K da equação (3.12), tem-se:

$$
\begin{aligned}
\mathrm{K}^{\prime} & =\left[\begin{array}{cc}
k \mathrm{I} & (1-k) \tilde{\mathbf{p}}_{0} \\
\mathbf{0}^{\mathrm{T}} & 1
\end{array}\right] \mathrm{K}=\left[\begin{array}{cc}
k \mathrm{I} & (1-k) \tilde{\mathbf{p}}_{0} \\
\mathbf{0}^{\mathrm{T}} & 1
\end{array}\right]\left[\begin{array}{cc}
\mathrm{A} & \tilde{\mathbf{p}}_{0} \\
\mathbf{0}^{\mathrm{T}} & 1
\end{array}\right]= \\
& =\left[\begin{array}{cc}
k \mathrm{~A} & \tilde{\mathbf{p}}_{0} \\
\mathbf{0}^{\mathrm{T}} & 1
\end{array}\right]=\mathrm{K}\left[\begin{array}{cc}
k \mathrm{I} & \\
& 1
\end{array}\right] .
\end{aligned}
$$

Portanto, o efeito de zoom por um fator $k$ é multiplicar a matriz de calibração $\mathrm{K}$ pela direita por $\operatorname{diag}(k, k, 1)$.

\subsubsection{Rotação de Câmera}

Considerando a rotação da câmera em torno do seu centro sem alteração dos parâmetros internos, se $\mathbf{x}$ e $\mathbf{x}^{\prime}$ são imagens do ponto $\mathbf{X}$ antes e depois da rotação pura, então:

$$
\mathbf{x}=\mathrm{K}[\mathrm{I} \mid \mathbf{0}] \mathbf{X} \quad \text { e } \quad \mathbf{x}^{\prime}=\mathrm{KR}[\mathrm{I} \mid \mathbf{0}] \mathbf{X}=\mathrm{KRK}^{-1} \mathrm{~K}[\mathrm{I} \mid \mathbf{0}] \mathbf{X}=\mathrm{KRK}^{-1} \mathbf{X} .
$$

Assim, $\mathbf{x}^{\prime}=\mathrm{Hx}$, com $\mathrm{H}=\mathrm{KRK}^{-1}$. Esta homografia é uma rotação conjugada (vide Apêndice A, Seção A.1), e tem os mesmos autovalores (em escala) que a matriz de rotação, $\left\{\mu, \mu \mathrm{e}^{\mathrm{i} \theta}, \mu \mathrm{e}^{-\mathrm{i} \theta}\right\}$, onde $\mu$ é um fator de escala desconhecido (se H é tal que $\operatorname{det}(\mathrm{H})=1$, então $\mu=1$ ). Conseqüentemente, o ângulo de rotação entre cenas pode ser calculado diretamente da fase dos autovalores complexos de H. Analogamente, o autovetor de $\mathrm{H}$ correspondente ao autovalor real é o ponto de fuga do eixo de rotação.

A Figura 3.10 mostra um exemplo do movimento de rotação em torno do centro de câmera, em comparação com o movimento de rotação combinado com a translação do centro de câmera. Entre as imagens da Figura 3.10(a) e da Figura 3.10(b) há rotação da câmera em torno do seu centro. Pontos corre- 


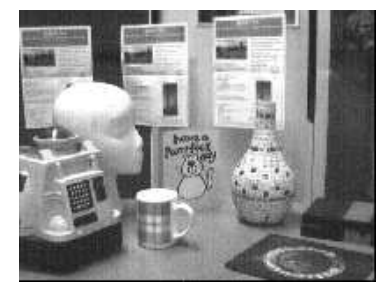

(a)

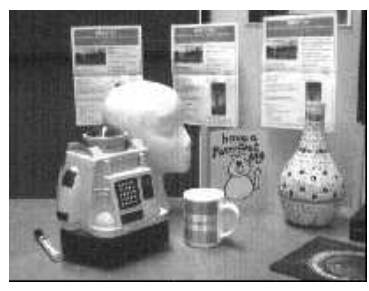

(b)

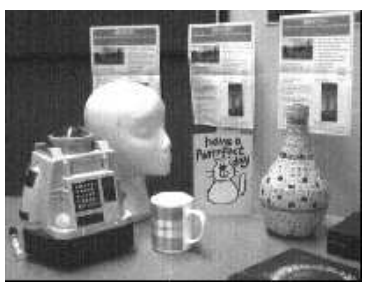

(c)

Figura 3.10: Exemplo de rotação e translação de câmera. (a) Posição inicial. (b) Posição após rotação. (c) Posição após rotação e translação.

spondentes das duas imagens (pontos que são imagens do mesmo ponto 3D) são relacionados por uma transformação projetiva planar. Nota-se que pontos 3D de diferentes profundidades cujas imagens coincidem na Figura 3.10(a) também têm imagens coincidentes na Figura 3.10(b), de forma que não há paralaxe. Contudo, entre as imagens da Figura 3.10(a) e da Figura 3.10(c), há rotação em torno do centro de câmera e translação. Segundo um movimento qualquer, pontos de profundidades diferentes com imagens coincidentes na Figura 3.10(a) têm imagens diferentes na Figura 3.10(c), de forma que há paralaxe. 


\section{Geometria Epipolar e Matriz Fundamental}

A geometria epipolar é a geometria projetiva inerente entre duas imagens, dependente apenas dos parâmetros internos das câmeras e suas posições relativas. A matriz fundamental, de dimensão $3 \times 3$ e de posto 2 , representa esta geometria intrínseca. Se um ponto do espaço 3D $\mathbf{X}$ tem imagem $\mathbf{x}$ em uma cena e $\mathbf{x}^{\prime}$ em outra, então estes pontos das imagens satisfazem a relação $\mathbf{x}^{\prime \mathrm{T}} \mathbf{F x}=0$. Pode-se determinar as câmeras que geram duas imagens a partir da matriz fundamental da geometria epipolar entre elas. As câmeras assim determinadas definem a reconstrução projetiva a partir das imagens e estão sujeitas a uma ambigüidade representada por uma transformação projetiva 3D. Se os parâmetros internos das câmeras são conhecidos, pode-se calcular o deslocamento Euclidiano entre elas, sob um número finito de ambigüidades. Por ser independente da estrutura da cena, pode-se calcular a matriz fundamental a partir de pontos correspondentes sobre as imagens, sem conhecimento do ponto do espaço 3D que representam, dos parâmetros internos das câmeras e da posição relativa entre elas.

Os conceitos apresentados neste capítulo foram extraídos de (HARTLEY; ZISSERMAN, 2000), (FAUGERAS, 1993), (ULLMAN, 1979), e (XU; ZHANG, 1996). A notação adotada e as ilustrações das figuras são de (HARTLEY; ZISSERMAN, 2000).

\subsection{Geometria Epipolar}

A geometria epipolar entre duas imagens de uma cena é essencialmente a geometria da intersecção dos planos de imagem com o feixe de planos cujo eixo é a reta base entre as imagens - a que passa pelos centros de câmera. A geometria epipolar pode ser analisada considerando pontos correspondentes em pares de imagens - tanto em pares estéreo como em pares de uma seqüência de imagens tomadas por uma câmera em movimento. Em ambos os casos, esta análise é idêntica, porém a visualização é mais simples para o caso de pares estéreo de câmeras. Dado um ponto 3D X, suas imagens segundo duas câmeras distintas são $\mathbf{x}$ para 


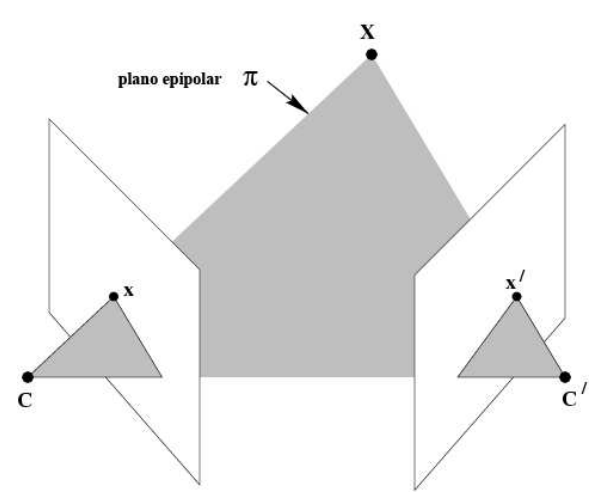

(a)

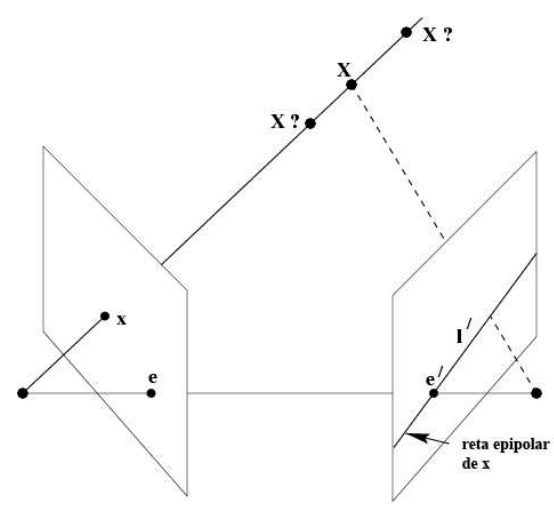

(b)

Figura 4.1: Geometria de Pontos Correspondentes. (a) Plano Epipolar. (b) Reta Epipolar.

a primeira e $\mathbf{x}^{\prime}$ para a segunda. Os pontos $\mathbf{X}, \mathbf{x}$ e $\mathbf{x}^{\prime}$ são coplanares e formam um plano denominado $\pi$, conforme ilustrado na Figura 4.1(a). Os raios das projeções inversas de $\mathbf{x}$ e $\mathbf{x}^{\prime}$ cruzam-se no ponto $\mathbf{X}$, sendo que estes raios são coplanares, pertencentes ao plano $\pi$. Esta propriedade tem grande importância para a determinação de pontos correspondentes entre as imagens. Supondo que apenas o ponto $\mathrm{x}$ é conhecido, determina-se o plano $\pi$ a partir da reta base e do raio da projeção inversa de $\mathbf{x}$. Como o raio da projeção inversa de $\mathbf{x}^{\prime}$ (desconhecido) também pertence a $\pi$, então $\mathbf{x}^{\prime}$ pertence à reta $\mathbf{l}^{\prime}$, formada pela intersecção entre $\pi$ e o segundo plano de imagem. Esta reta $\mathbf{l}^{\prime}$ é a imagem do raio da projeção inversa de $\mathbf{x}$ na segunda cena. Desta forma, o ponto correspondente a $\mathbf{x}$ está restrito à reta $\mathbf{l}^{\prime}$, conforme ilustrado na Figura 4.1(b).

As entidades geométricas presentes na geometria epipolar são o epipolo, o plano epipolar e a reta epipolar. O epipolo é o ponto de intersecção da reta base com o plano de imagem. De maneira equivalente, o epipolo é a projeção do centro de câmera de uma imagem na outra. Um plano epipolar é um plano que contém a reta base. Existe um feixe de planos epipolares determinado pela reta base. Uma reta epipolar é a intersecção de um plano epipolar com o plano de imagem. Todas as retas epipolares de uma imagem passam pelo epipolo desta imagem. A Figura 4.2 ilustra estes elementos.

\subsection{Matriz Fundamental}

A matriz fundamental é a representação algébrica da geometria epipolar entre duas imagens, e pode ser deduzida da relação entre um ponto em uma imagem e sua reta epipolar na outra imagem. Na Figura 4.1 vê-se que para cada ponto $\mathbf{x}$ 


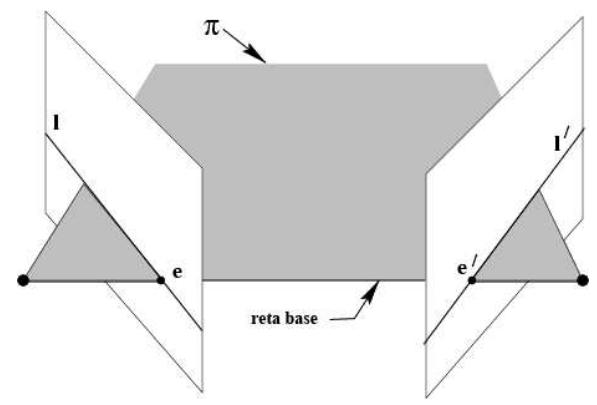

(a)

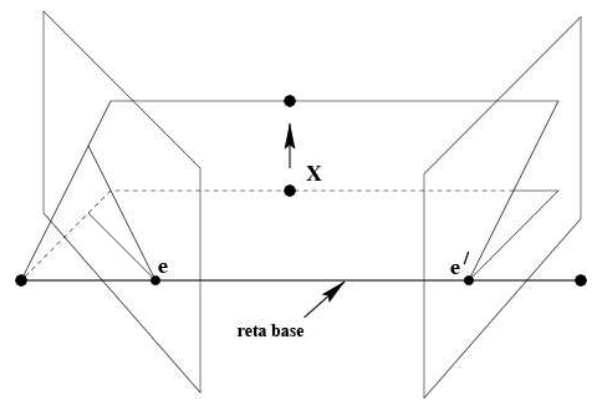

(b)

Figura 4.2: Geometria Epipolar. (a) Relação entre plano epipolar, reta base, epipolos e retas epipolares. (b) Feixe de planos epipolares.

em uma imagem existe uma reta epipolar $\mathbf{l}^{\prime}$ na outra imagem, e qualquer ponto $\mathbf{x}^{\prime}$ da segunda imagem correspondente a $\mathbf{x}$ deve pertencer à reta epipolar $\mathbf{l}^{\prime}$. A reta epipolar é a projeção na segunda imagem do raio que parte do ponto $\mathbf{x}$ e passa pelo centro de câmera $\mathbf{C}$ da primeira imagem. Desta forma, estabelece-se uma relação $\mathbf{x} \mapsto \mathbf{l}^{\prime}$ entre um ponto de uma imagem e a reta epipolar correspondente na outra imagem. Esta relação é uma correlação (singular), que representa uma transformação projetiva de pontos para retas, representada por uma matriz denominada matriz fundamental.

\subsubsection{Dedução Geométrica}

Considerando um plano $\pi$ que não contenha nenhum dos centros de câmera, o ponto $\mathbf{X}$ é a intersecção deste plano com o raio passando pelo primeiro centro de câmera e pelo ponto $\mathbf{x}$ da primeira imagem. Como $\mathbf{X}$ está sobre o raio correspondente a $\mathbf{x}$, o ponto $\mathbf{x}^{\prime}$ projetado na segunda imagem deve estar sobre a reta epipolar $\mathbf{l}^{\prime}$, conforme ilustrado na Figura 4.1(b). Os pontos $\mathbf{x}$ e $\mathbf{x}^{\prime}$ são ambos imagens do ponto 3D $\mathbf{X}$. O conjunto de todos os pontos $\mathbf{x}_{i}$ da primeira imagem e os correspondentes $\mathbf{x}_{i}^{\prime}$ da segunda imagem são projetivamente equivalentes, uma vez que cada um é projetivamente equivalente ao ponto $\mathbf{X}_{i}$ pertencente ao plano $\pi$. Assim, define-se uma homografia $2 \mathrm{D}_{\pi}$ que mapeia cada $\mathbf{x}_{i}$ ao seu correspondente $\mathbf{x}_{i}^{\prime}$, conforme ilustrado na Figura 4.3.

A reta epipolar $\mathbf{l}^{\prime}$ passando por um ponto $\mathbf{x}^{\prime}$ e pelo epipolo $\mathbf{e}^{\prime}$ é dada por $\mathbf{l}^{\prime}=\mathbf{e}^{\prime} \times \mathbf{x}^{\prime}=\left[\mathbf{e}^{\prime}\right]_{\times} \mathbf{x}^{\prime}$ (a notação $[\mathbf{a}]_{\times} \mathbf{b}$ denota o produto vetorial $\mathbf{a} \times \mathbf{b}$, detalhada no Apêndice A, Seção A.2.1). Como $\mathbf{x}^{\prime}=\mathrm{H}_{\pi} \mathbf{x}$, tem-se:

$$
\mathbf{l}^{\prime}=\left[\mathbf{e}^{\prime}\right]_{\times} \mathrm{H}_{\pi} \mathbf{x}=\mathbf{F} \mathbf{x}
$$




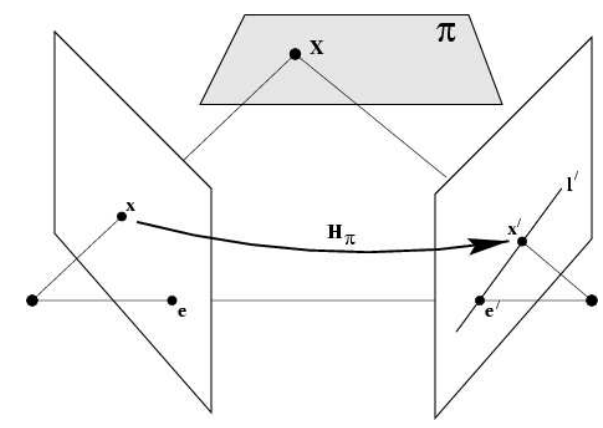

Figura 4.3: Homografia 2D entre pontos correspondentes.

Assim, a matriz fundamental $\mathrm{F}$ pode ser escrita como $\mathrm{F}=\left[\mathbf{e}^{\prime}\right]_{\times} \mathrm{H}_{\pi}$, onde $\mathrm{H}_{\pi}$ é a relação de transferência de uma imagem para a outra através de um plano $\pi$ qualquer. Ademais, como $\left[\mathbf{e}^{\prime}\right]_{\times}$tem posto 2 e $\mathrm{H}_{\pi}$ tem posto $3, \mathrm{~F}$ é uma matriz singular de posto 2. Geometricamente, F representa uma relação entre o plano projetivo bidimensional $\mathbf{P}^{2}$ da primeira imagem e o feixe de retas epipolares passando por $\mathbf{e}^{\prime}$. Desta forma, F representa um mapeamento do espaço projetivo bidimensional para o espaço projetivo unidimensional, e portanto tem posto 2 .

\subsubsection{Dedução Algébrica}

A matriz fundamental pode ser determinada algebricamente a partir das matrizes de câmera P e P', conforme formulado por Xu e Zhang (XU; ZHANG, 1996). O raio da projeção inversa de $\mathbf{x}$ a partir de $\mathrm{P}$ é determinado através da solução de $\mathrm{PX}=\mathbf{x}$. O conjunto de soluções desta equação é dada pela equação (3.16):

$$
\mathbf{X}(\lambda)=\mathrm{P}^{+} \mathbf{x}+\lambda \mathbf{C}
$$

onde a matriz $\mathrm{P}^{+}$é a pseudo-inversa de $\mathrm{P}$, de forma que $\mathrm{PP}^{+}=\mathrm{I}$, e o centro de câmera $\mathbf{C}$ é o espaço nulo de $\mathrm{P}$, definido por $\mathbf{P C}=\mathbf{0}$. Dois pontos particulares, $\mathrm{P}^{+} \mathbf{x}($ para $\lambda=0)$ e o centro de câmera $\mathbf{C}($ para $\lambda \rightarrow \infty)$, são projetados pela segunda câmera $\mathrm{P}^{\prime}$ respectivamente $e m \mathrm{P}^{\prime} \mathrm{P}^{+} \mathrm{x}$ e $\mathrm{P}^{\prime} \mathbf{C}$ na segunda imagem. A reta epipolar correspondente a $\mathbf{x}$ é a reta que passa por estes dois pontos, $\mathbf{l}^{\prime}=\left(\mathrm{P}^{\prime} \mathbf{C}\right) \times$ $\left(\mathrm{P}^{\prime} \mathrm{P}^{+} \mathbf{x}\right)$. O ponto $\mathrm{P}^{\prime} \mathbf{C}$ é o epipolo $\mathbf{e}^{\prime}-$ a projeção do centro de câmera da primeira câmera na segunda imagem. Assim, $\mathbf{l}^{\prime}=\left[\mathbf{e}^{\prime}\right]_{\times}\left(\mathrm{P}^{\prime} \mathrm{P}^{+}\right) \mathbf{x}=\mathrm{Fx}$, onde $\mathrm{F}$ é a dada por:

$$
\mathrm{F}=\left[\mathbf{e}^{\prime}\right]_{\times}\left(\mathrm{P}^{\prime} \mathrm{P}^{+}\right)
$$

Esta é essencialmente a mesma formulação da matriz fundamental que a obtida anteriormente, com $\mathrm{H}_{\pi}=\mathrm{P}^{\prime} \mathrm{P}^{+}$. No entanto, esta formulação não é válida 
no caso em que as câmeras têm o mesmo centro de câmera, onde $\mathbf{P}^{\prime} \mathbf{C}=\mathbf{0}$, e a matriz $\mathrm{F}$ definida por (4.3) é uma matriz $3 \times 3$ nula.

Considerando matrizes de câmera correspondentes a um par estéreo calibrado, onde o sistema de coordenadas global coincidente com o sistema de coordenadas de câmera da primeira câmera, segundo (3.6) e (3.10) tem-se:

$$
\mathrm{P}=\mathrm{K}[\mathrm{I} \mid \mathbf{0}] \quad \text { e } \quad \mathrm{P}^{\prime}=\mathrm{K}^{\prime}[\mathrm{R} \mid \mathbf{t}] .
$$

onde $\mathrm{K}$ e K' são respectivamente as matrizes de calibração da primeira e da segunda câmera, e R e t são respectivamente a rotação e a translação relativas da primeira para a segunda câmera. Como $\mathrm{PP}^{+}=\mathrm{I}$ e $\mathrm{PC}=\mathbf{0}$, tem-se:

$$
\mathrm{P}^{+}=\left[\begin{array}{c}
\mathrm{K}^{-1} \\
\mathbf{0}^{\mathrm{T}}
\end{array}\right] \quad \text { e } \quad \mathrm{C}=\left(\begin{array}{l}
\mathbf{0} \\
1
\end{array}\right)
$$

Assim, (vide detalhes no Apêndice A, Seção A.2.2) segue que:

$$
\begin{aligned}
\mathrm{F} & =\left[\mathrm{P}^{\prime} \mathbf{C}\right]_{\times} \mathrm{P}^{\prime} \mathrm{P}^{+}= \\
& =\left[\mathrm{K}^{\prime} \mathbf{t}\right]_{\times} \mathrm{K}^{\prime} \mathrm{RK}^{-1}=\mathrm{K}^{\prime-\mathrm{T}}[\mathbf{t}]_{\times} \mathrm{RK}^{-1}=\mathrm{K}^{\prime-\mathrm{T}} \mathrm{R}\left[\mathrm{R}^{\mathrm{T}} \mathbf{t}\right]_{\times} \mathrm{K}^{-1}=\mathrm{K}^{\prime-\mathrm{T}} \mathrm{RK}^{\mathrm{T}}\left[\mathrm{KR}^{\mathrm{T}} \mathbf{t}\right]_{\times} .
\end{aligned}
$$

Os epipolos são dados por:

$$
\mathbf{e}=\mathrm{P}\left(\begin{array}{c}
-\mathrm{R}^{\mathrm{T}} \mathbf{t} \\
1
\end{array}\right)=\mathrm{KR}^{\mathrm{T}} \mathbf{t} \quad \text { e } \quad \mathbf{e}^{\prime}=\mathrm{P}^{\prime}\left(\begin{array}{l}
\mathbf{0} \\
1
\end{array}\right)=\mathrm{K}^{\prime} \mathbf{t}
$$

Assim, a equação (4.6) pode ser reescrita como:

$$
\mathrm{F}=\left[\mathbf{e}^{\prime}\right]_{\times} \mathrm{K}^{\prime} \mathrm{RK}^{-1}=\mathrm{K}^{\prime-\mathrm{T}}[\mathbf{t}]_{\times} \mathrm{RK}^{-1}=\mathrm{K}^{\prime-\mathrm{T}} \mathrm{R}\left[\mathrm{R}^{\mathrm{T}} \mathrm{t}\right]_{\times} \mathrm{K}^{-1}=\mathrm{K}^{\prime-\mathrm{T}} \mathrm{RK}^{\mathrm{T}}[\mathbf{e}]_{\times}
$$

\subsubsection{Condição de Correspondência}

A principal propriedade da matriz fundamental é a chamada condição de correspondência: para qualquer par de pontos correspondentes $\mathbf{x} \leftrightarrow \mathbf{x}^{\prime}$ em duas imagens, a matriz fundamental satisfaz a condição $\mathbf{x}^{\prime \mathrm{T}} \mathrm{F} \mathbf{x}=0$.

Se os pontos $\mathbf{x}$ e $\mathbf{x}^{\prime}$ são correspondentes, então $\mathbf{x}^{\prime}$ está sobre a reta epipolar $\mathbf{l}^{\prime}=\mathbf{F} \mathbf{x}$ correspondente ao ponto $\mathbf{x}$. Assim, $\mathbf{x}^{\prime \mathrm{T}} \mathbf{l}^{\prime}=0$, e portanto $\mathbf{x}^{\prime \mathrm{T}} \mathbf{F} \mathbf{x}=0$. Por 
outro lado, se os pontos das imagens satisfazem $\mathbf{x}^{\prime \mathrm{T}} \mathrm{F} \mathbf{x}=0$, os raios definidos por estes pontos são coplanares, o que é condição suficiente para que os pontos sejam correspondentes.

Esta propriedade permite caracterizar a matriz fundamental sem conhecimento das matrizes de câmera, apenas em termos de pontos correspondentes nas imagens. Desta forma, pode-se calcular F a partir da correspondência entre as imagens. Deve-se ainda determinar quantos pares de pontos correspondentes são necessários para se calcular F, e quais as condições para que a matriz seja exatamente determinada por estas correspondências.

\subsubsection{Propriedades da Matriz Fundamental}

Dadas duas imagens obtidas a partir de câmeras com centros distintos, a matriz fundamental $\mathrm{F}$ é a única matriz homogênea $3 \times 3$ de posto 2 que, para todos pontos correspondentes $\mathbf{x} \leftrightarrow \mathbf{x}^{\prime}$, satisfaz a condição:

$$
\mathrm{x}^{\prime \mathrm{T}} \mathrm{Fx}=0
$$

Transposta. Se F é a matriz fundamental para um par de câmeras ( $\left.P, \mathrm{P}^{\prime}\right)$, então $\mathrm{F}^{\mathrm{T}}$ é a matriz fundamental do par na ordem inversa $\left(\mathrm{P}^{\prime}, \mathrm{P}\right)$. De fato, se $\mathbf{x}^{\prime \mathrm{T}} \mathrm{F} \mathbf{x}=0$, então $\left(\mathbf{x}^{\prime \mathrm{T}} \mathrm{F} \mathbf{x}\right)^{\mathrm{T}}=0$. Segue que $\mathbf{x}^{\mathrm{T}} \mathrm{F}^{\mathrm{T}} \mathbf{x}^{\prime}=0$ que representa a condição (4.6) para o caso em que a primeira imagem é gerada por $\mathrm{P}^{\prime}$ e a segunda por $\mathrm{P}$.

Retas Epipolares. A qualquer ponto $\mathbf{x}$ na primeira imagem corresponde uma reta epipolar $\mathbf{l}^{\prime}=\mathbf{F x}$ na segunda imagem. Analogamente, $\mathbf{l}=\mathrm{F}^{\mathrm{T}} \mathbf{x}^{\prime}$ representa a reta epipolar na primeira imagem correspondente ao ponto $\mathbf{x}^{\prime}$ na segunda imagem.

Epipolos. Dado um ponto $\mathbf{x}$ qualquer da primeira imagem (diferente do epipolo $\mathbf{e}$ ), a reta epipolar $\mathbf{l}^{\prime}=\mathbf{F} \mathbf{x}$ contém o epipolo $\mathbf{e}^{\prime}$ na segunda imagem, de forma que $\mathbf{e}^{\prime \mathrm{T}}(\mathrm{F} \mathbf{x})=\left(\mathbf{e}^{\prime \mathrm{T}} \mathrm{F}\right) \mathbf{x}=0$, para todo $\mathbf{x}$. Segue que $\mathbf{e}^{\prime \mathrm{T}} \mathrm{F}=0$, ou seja $\mathbf{e}^{\prime}$ é o espaço nulo à esquerda de $\mathrm{F}$ (ou $\mathbf{e}^{\prime}$ é espaço nulo à direita de $\mathrm{F}^{\mathrm{T}}$ ). Analogamente, $\mathrm{Fe}=0$, portanto e é o espaço nulo à direita de $\mathrm{F}$.

Graus de Liberdade. A matriz fundamental $\mathrm{F}$ tem sete graus de liberdade. Uma matriz homogênea $3 \times 3$ tem oito razões independentes (nove elementos e um fator de escala), porém $F$ satisfaz a condição $\operatorname{det}(F)=0$ (F tem posto 2$)$, o que remove um grau de liberdade.

Correlação. A matriz fundamental F é uma correlação - uma transformação 


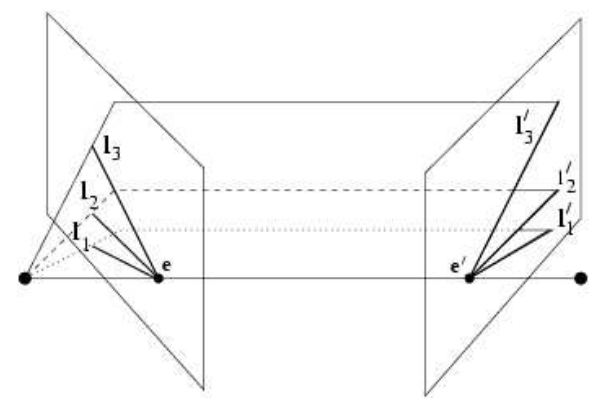

(a)

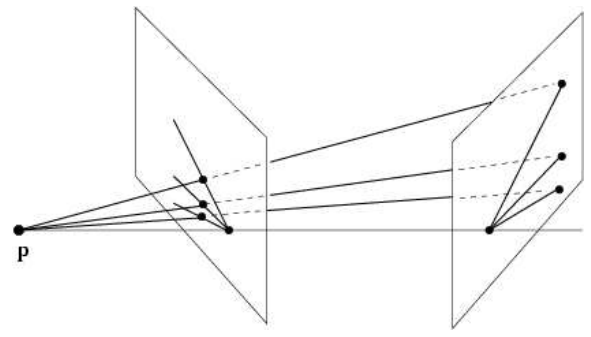

(b)

Figura 4.4: Homografia da Reta Epipolar. (a) Feixes de retas epipolares. (b) Transformação perspectiva entre retas epipolares.

projetiva que leva pontos a retas. Neste caso um ponto $\mathbf{x}$ na primeira imagem define uma reta $\mathbf{l}^{\prime}$ na segunda imagem, denominada a reta epipolar de $\mathbf{x}$. Se $\mathbf{l}$ e $\mathbf{l}^{\prime}$ são retas epipolares correspondentes, então qualquer ponto $\mathbf{x}$ pertencente a $\mathbf{l}$ é mapeado na mesma reta $\mathbf{l}^{\prime}$, conforme pode-se observar na Figura 4.4(b). Desta forma, não existe a relação inversa de F, que não tem posto completo.

Homografia da Reta Epipolar. O conjunto das retas epipolares em cada imagem forma um feixe de retas passando pelo epipolo, conforme ilustrado na Figura 4.4(a). Tal feixe de retas pode ser considerado um espaço projetivo unidimensional. Pode-se observar na Figura 4.4(b), que as retas epipolares correspondentes estão relacionadas por uma perspectiva, de forma que existe uma homografia entre o feixe de retas epipolares centradas em e na primeira imagem, e o feixe centrado em $\mathbf{e}^{\prime}$ na segunda. Uma homografia entre dois espaços projetivos unidimensionais tem três graus de liberdade.

Os graus de liberdade da matriz fundamental podem ser considerados como dois relativos a e, dois relativos a $\mathbf{e}^{\prime}$, e três relativos à homografia da reta epipolar, que relaciona uma reta passando por e com uma reta correspondente passando por $\mathbf{e}^{\prime}$.

Supondo $\mathbf{l}$ e $\mathbf{l}^{\prime}$ retas epipolares correspondentes, e $\mathbf{k}$ uma reta qualquer que não contém o epipolo e, então $\mathbf{l}^{\prime}=\mathrm{F}[\mathbf{k}]_{\times} \mathbf{l}$. De fato, o ponto de intersecção das retas $\mathbf{k}$ e $\mathbf{l}$ é $\mathbf{x}=\mathbf{k} \times \mathbf{l}$, e a reta epipolar correspondente a $\mathbf{x}$ é $\mathbf{l}^{\prime}=F \mathbf{x}=F[\mathbf{k}]_{\times} \mathbf{l}$. Uma escolha conveniente é $\mathbf{k}=\mathbf{e}$, pois $\mathbf{k}^{\mathrm{T}} \mathbf{e}=\mathbf{e}^{\mathrm{T}} \mathbf{e} \neq 0$, de forma que a reta $\mathbf{k}$ não contém o ponto e. Analogamente, pode-se escolher $\mathbf{k}^{\prime}=\mathbf{e}^{\prime}$. Assim, a homografia da reta epipolar pode ser escrita como:

$$
\mathbf{l}^{\prime}=\mathrm{F}[\mathbf{e}]_{\times} \mathbf{l} \quad \text { e } \quad \mathbf{l}=\mathrm{F}^{\mathrm{T}}\left[\mathbf{e}^{\prime}\right]_{\times} \mathbf{l}^{\prime} .
$$




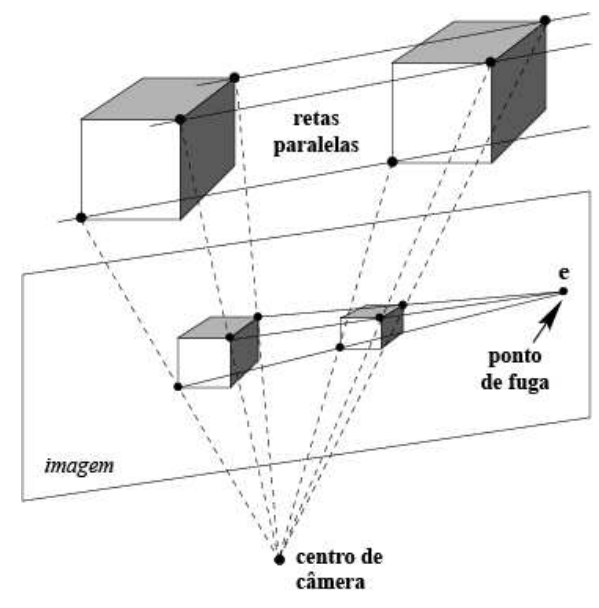

Figura 4.5: Epipolos do movimento de translação pura da câmera.

\subsection{Movimentos Especiais de Câmera}

Um movimento especial de câmera é aquele em que há uma relação particular entre a direção de translação t e a direção do eixo de rotação a. Dois casos são de especial interesse: movimento de translação pura, onde não há rotação, e o movimento planar puro, onde t é ortogonal a a. Tais movimentos são considerados puros quando não há alteração dos parâmetros internos da câmera. Estes casos são importantes pois ocorrem realmente na prática e a matriz fundamental apresenta uma forma especial e tem propriedades adicionais para cada um deles.

\subsubsection{Movimento de Translação Pura}

No movimento de translação pura de uma câmera na direção t, pontos do espaço tridimensional movem-se sobre retas paralelas a t, e a intersecção das imagens destas retas paralelas é o ponto $\mathbf{v}=\mathbf{e}=\mathbf{e}^{\prime}$ na direção de $\mathbf{t}$, conforme ilustrado na Figura 4.5. Segundo o movimento de translação pura de uma câmera, pontos 3D aparentam deslizar sobre retas paralelas. As imagens destas retas paralelas encontram-se em um ponto de fuga correspondente à direção de translação, e que corresponde ao epipolo e, idêntico em ambas imagens. O ponto $\mathbf{v}$ coincide com o epipolo de ambas imagens, e as imagens das retas paralelas a $\mathbf{t}$ são as retas epipolares.

Considerando que uma câmera realiza movimento de translação pura sem rotação e sem alteração de seus parâmetros internos, pode-se assumir $P=K[I \mid 0]$ e $\mathrm{P}^{\prime}=\mathrm{K}^{\prime}[\mathrm{R} \mid \mathbf{t}]$, onde $\mathrm{R}=\mathrm{I}$ e $\mathrm{K}^{\prime}=\mathrm{K}$. Pela equação (4.8), tem-se: 


$$
\mathrm{F}=\left[\mathbf{e}^{\prime}\right]_{\times} \mathrm{KK}^{-1}=\left[\mathbf{e}^{\prime}\right]_{\times}
$$

Dado o ponto $\mathbf{x}=(x, y, 1)^{\mathrm{T}}$ da imagem, tem-se $\mathbf{x}=\mathrm{PX}=\mathrm{K}[\mathrm{I} \mid \mathbf{0}] \mathbf{X}$, e as coordenadas não-homogêneas do ponto $3 \mathrm{D}$ são $(\mathrm{X}, \mathrm{Y}, \mathrm{Z})^{\mathrm{T}}=\mathrm{K}^{-1} \mathrm{x} / \mathrm{Z}$, onde $\mathrm{Z}$ é a profundidade do ponto $\mathbf{X}$ (distância de $\mathbf{X}$ ao centro de câmera medida na direção do eixo principal da primeira câmera). Como $\mathbf{x}^{\prime}=\mathrm{P}^{\prime} \mathbf{X}=\mathrm{K}[\mathrm{I} \mid \mathbf{t}] \mathbf{X}$, a relação entre os pontos $\mathbf{x}$ e $\mathbf{x}^{\prime}$ é dada por:

$$
\mathrm{x}^{\prime}=\mathrm{x}+\mathrm{Kt} / \mathrm{Z}
$$

A equação (4.12) mostra que o movimento do ponto da imagem parte de $\mathbf{x}$ e desloca-se ao longo da reta definida por $\mathbf{x}$ e pelo epipolo $\mathbf{v}=\mathbf{e}=\mathbf{e}^{\prime}$. A extensão deste movimento depende do módulo da translação $\mathbf{t}$ (vetor não-homogêneo) e da profundidade $\mathrm{z}$, de forma que a imagem de um ponto 3D mais próximo à câmera parece mover-se mais rapidamente que a imagem de um ponto 3D mais distante.

No caso do movimento de translação pura, $F=\left[\mathbf{e}^{\prime}\right]_{\times}$é anti-simétrica e tem apenas 2 graus de liberdade, correspondentes ao epipolo. A reta epipolar de $\mathbf{x}$ é $\mathbf{l}^{\prime}=\mathbf{F} \mathbf{x}=[\mathbf{e}]_{\times} \mathbf{x}$, e $\mathbf{x}$ também pertence a esta reta, pois $\mathbf{x}^{\mathrm{T}}[\mathbf{e}]_{\times} \mathbf{x}=0$. Como e e $\mathbf{e}^{\prime}$ são colineares, o movimento de transação pura é dito auto-epipolar.

\subsubsection{Movimento Geral}

Dadas duas câmeras quaisquer, pode-se rotacionar a primeira delas de forma que fique alinhada com a segunda. Esta rotação equivale a uma transformação projetiva aplicada à primeira imagem. Pode-se ainda corrigir a primeira imagem de forma a eliminar as diferenças das matrizes de calibração das duas câmeras. A combinação destas duas correções representa uma transformação projetiva $\mathrm{H}$ da primeira imagem. Após estas correções, a relação entre as câmeras será uma translação pura. A matriz fundamental correspondente ao par formado pela primeira imagem corrigida e pela segunda imagem será $\hat{F}=\left[\mathbf{e}^{\prime}\right]_{\times}$, e satisfaz $\mathbf{x}^{\prime \mathrm{T}} \hat{\mathrm{F}} \hat{\mathbf{x}}$, onde $\hat{\mathbf{x}}$ é o ponto da primeira imagem corrigido. Assim, a matriz fundamental dos pontos correspondentes originais $\mathbf{x} \leftrightarrow \mathbf{x}^{\prime}$, é $\mathbf{F}=\left[\mathbf{e}^{\prime}\right]_{\times} \mathrm{H}$, conforme ilustrado na Figura 4.6.

Considerando que as duas câmeras são $\mathrm{P}=\mathrm{K}[\mathrm{I} \mid \mathbf{0}]$ e $\mathrm{P}^{\prime}=\mathrm{K}^{\prime}[\mathrm{R} \mid \mathbf{t}]$, a transformação projetiva que corrige a primeira câmera é $\mathrm{H}=\mathrm{K}^{\prime} \mathrm{RK}^{-1}$ (vide Seção 3.4.2), e $\mathrm{F}=\left[\mathbf{e}^{\prime}\right]_{\times} \mathrm{H}=\left[\mathbf{e}^{\prime}\right]_{\times} \mathrm{K}^{\prime} \mathrm{RK}^{-1}$. 


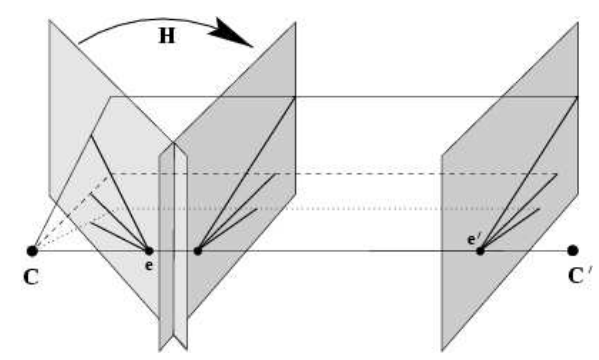

Figura 4.6: Movimento Geral de Câmera.

Analogamente ao caso do movimento de translação pura, dado o ponto $\mathbf{x}=$ $(x, y, 1)^{\mathrm{T}}$ da imagem, tem-se $\mathbf{x}=\mathrm{PX}=\mathrm{K}[\mathrm{I} \mid \mathbf{0}] \mathbf{X}$, e as coordenadas não-homogêneas do ponto $3 \mathrm{D}$ são $(\mathrm{X}, \mathrm{Y}, \mathrm{Z})^{\mathrm{T}}=\mathrm{K}^{-1} \mathbf{X} / \mathrm{Z}$, onde $\mathrm{Z}$ é a profundidade do ponto $\mathbf{X}$. Como $\mathbf{x}^{\prime}=\mathrm{P}^{\prime} \mathbf{X}=\mathrm{K}[\mathrm{R} \mid \mathbf{t}] \mathbf{X}$, a relação entre os pontos $\mathbf{x}$ e $\mathbf{x}^{\prime}$ é dada por:

$$
\mathbf{x}^{\prime}=\mathrm{K}^{\prime} \mathrm{RK}^{-1} \mathbf{x}+\mathrm{K}^{\prime} \mathbf{t} / \mathrm{Z}
$$

Esta relação é composta de duas partes: a primeira depende apenas da posição do ponto da imagem e é independente da profundidade - representando a rotação da câmera e alteração dos parâmetros internos; a segunda depende apenas da profundidade, e é independente da posição do ponto da imagem - representando a translação.

\subsubsection{Movimento planar puro}

Dadas duas câmeras de mesmos parâmetros intrínsecos $\mathrm{P}=\mathrm{K}[\mathrm{I} \mid \mathbf{0}]$ e $\mathrm{P}^{\prime}=\mathrm{K}[\mathrm{R} \mid \mathbf{t}]$, o movimento planar puro caracteriza-se por apresentar o eixo de rotação a ortogonal à direção de translação t. A restrição de ortogonalidade entre o eixo de rotação e a direção de translação implica que $[\mathbf{t}]_{\times}$tem apenas 1 grau de liberdade (vide detalhes no Apêndice A, Seção A.3). Como $F=K^{\prime-T}[\mathbf{t}]_{\times} R K^{-1}$ e a matriz de parâmetros internos da câmera tem 5 graus de liberdade, a matriz fundamental F passa a ter 6 graus de liberdade.

Três casos particulares de movimento planar puro são definidos considerandose a direção de translação paralela a um dos planos formados pelos eixos do sistema de coordenadas global, XZ, YZ e XY, e o sistema de coordenadas de câmera da primeira câmera coincidente com o sistema de coordenadas global. Nestes casos, para $\mathrm{F}=\mathrm{K}^{\prime-\mathrm{T}}[\mathbf{t}]_{\times} \mathrm{RK}^{-1}$, tem-se: 


$$
\begin{aligned}
& \mathbf{t} \in \mathrm{XZ} \rightarrow \mathrm{F}=\mathrm{K}^{-\mathrm{T}}\left[\begin{array}{ccc}
0 & -z & 0 \\
z \cos \theta+x \sin \theta & 0 & -x \cos \theta+z \sin \theta \\
0 & x & 0
\end{array}\right] \mathrm{K}^{-1}, \\
& \mathbf{t} \in \mathrm{YZ} \rightarrow \mathrm{F}=\mathrm{K}^{-\mathrm{T}}\left[\begin{array}{ccc}
0 & -z \cos \theta+y \sin \theta & y \cos \theta+z \sin \theta \\
z & 0 & 0
\end{array}\right] \mathrm{K}^{-1}, \\
& \mathbf{t} \in \mathrm{XY} \rightarrow \mathrm{F}=\mathrm{K}^{-\mathrm{T}}\left[\begin{array}{cccc}
-y & 0 & 0 & y \\
0 & 0 & 0 & -x \\
-y \cos \theta+x \sin \theta & x \cos \theta+y \sin \theta & 0
\end{array}\right] \mathrm{K}^{-1} .
\end{aligned}
$$

\subsection{Matrizes de Câmera}

As relações $\mathbf{l}^{\prime}=\mathrm{Fx}$ e $\mathbf{x}^{\prime \mathrm{T}} \mathrm{F} \mathbf{x}=0$ da matriz fundamental $\mathrm{F}$ são projetivas, dependendo apenas das coordenadas projetivas das imagens. Portanto, a relação entre as imagens é um invariante projetivo: dada uma transformação projetiva sobre as coordenadas de imagem $\hat{\mathbf{x}}=H \mathbf{x}$ e $\hat{\mathbf{x}}^{\prime}=H^{\prime} \mathbf{x}^{\prime}$, existe uma relação $\hat{\mathbf{l}}=\hat{\mathbf{F}} \hat{\mathbf{x}}$, onde $\hat{\mathrm{F}}=\mathrm{H}^{-\mathrm{T}} \mathrm{FH}^{-1}$ é a matriz fundamental correspondente. Analogamente, a matriz fundamental $\mathrm{F}$ depende apenas das propriedades projetivas das câmeras P e P'.

Uma matriz de câmera relaciona coordenadas 3D a coordenadas de imagem, e depende tanto da escolha do sistema de coordenadas global como do sistema de coordenadas de imagem. No entanto, F independe do sistema de coordenadas global, e é constante sob transformações projetivas do espaço 3D: se $\mathrm{H}$ é uma matriz $4 \times 4$ representando uma transformação projetiva do espaço 3D, então a matriz fundamental correspondente ao par de câmeras $\left(\mathrm{P}, \mathrm{P}^{\prime}\right)$ é idêntica à matriz fundamental representando o par de câmeras $\left(\mathrm{PH}, \mathrm{P}^{\prime} \mathrm{H}\right)$. De fato, observa-se que $\mathrm{PX}=(\mathrm{PH})\left(\mathrm{H}^{-1} \mathbf{X}\right)$ e $\mathrm{P}^{\prime} \mathbf{X}=\left(\mathrm{P}^{\prime} \mathrm{H}\right)\left(\mathrm{H}^{-1} \mathbf{X}\right)$, e se $\mathbf{X} \leftrightarrow \mathbf{X}^{\prime}$ são pontos correspondentes segundo o par de câmeras $\left(\mathrm{P}, \mathrm{P}^{\prime}\right)$, referentes ao ponto $3 \mathrm{D} \mathbf{X}$, então $\mathbf{x} \leftrightarrow \mathbf{x}^{\prime}$ também são pontos correspondentes segundo o par de câmeras (PH, $\left.\mathrm{P}^{\prime} \mathrm{H}\right)$, referentes ao ponto $3 \mathrm{D} \mathrm{H}^{-1} \mathbf{X}$. Assim, um par de câmeras $\left(\mathrm{P}, \mathrm{P}^{\prime}\right)$ determina exatamente a matriz fundamental $\mathrm{F}$, mas a matriz fundamental determina um par de matrizes de câmera a menos de uma transformação projetiva.

\subsubsection{Forma Canônica da Matriz de Câmera}

Costuma-se definir uma forma canônica para o par de câmeras correspondente a uma matriz fundamental. A primeira câmera $P=[I \mid \mathbf{0}]$ está centrada na ori- 
gem do sistema de coordenadas global e o eixo da coordenada $\mathrm{Z}$ coincide com o eixo principal da câmera. A segunda câmera $\mathrm{P}^{\prime}=[\mathrm{M} \mid \mathbf{m}]$ engloba o movimento de rotação e translação necessário para levar a primeira câmera à posição da segunda câmera, combinada à variação dos parâmetros intrínsecos. Um resultado importante é que a matriz fundamental correspondente a um par de matrizes de câmera $\mathrm{P}=[\mathrm{I} \mid \mathbf{0}]$ e $\mathrm{P}^{\prime}=[\mathrm{M} \mid \mathbf{m}]$ é $\mathrm{F}=[\mathbf{m}]_{\times} \mathrm{M}$.

\subsubsection{Ambigüidade Projetiva}

Conforme mencionado anteriormente, pares de matrizes de câmera que diferem por uma transformação projetiva geram a mesma matriz fundamental. Demonstrase que a matriz fundamental determina um par de matrizes de câmera a menos de uma transformação projetiva, de forma que a matriz fundamental representa a relação projetiva entre as duas câmeras. Seja F uma matriz fundamental e sejam $\left(\mathrm{P}, \mathrm{P}^{\prime}\right)$ e $\left(\tilde{\mathrm{P}}, \tilde{\mathrm{P}}^{\prime}\right)$ dois pares de matrizes de câmera tais que $\mathrm{F}$ seja a matriz fundamental correspondente a ambos. Então existe uma matriz $\mathrm{H} 4 \times 4$ não-singular tal que $\tilde{\mathrm{P}}=\mathrm{PH}$ e $\tilde{\mathrm{P}}^{\prime}=\mathrm{P}^{\prime} \mathrm{H}$.

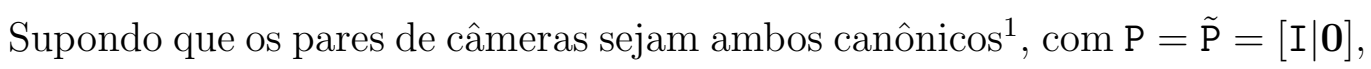
$\mathrm{P}^{\prime}=[\mathrm{A} \mid \mathbf{a}]$ e $\tilde{\mathrm{P}}^{\prime}=[\tilde{\mathrm{A}} \mid \tilde{\mathbf{a}}]$, a matriz fundamental é $\mathrm{F}=[\mathbf{a}]_{\times} \mathrm{A}=[\tilde{\mathbf{a}}]_{\times} \tilde{\mathrm{A}}$. Nota-se que $\mathbf{a}^{\mathrm{T}} \mathrm{F}=\mathbf{a}^{\mathrm{T}}[\mathbf{a}]_{\times} \mathrm{A}=0$ e $\tilde{\mathbf{a}}^{\mathrm{T}} \mathrm{F}=\tilde{\mathbf{a}}^{\mathrm{T}}[\tilde{\mathbf{a}}]_{\times} \tilde{\mathrm{A}}=0$. Como $\mathrm{F}$ tem posto 2 , segue que $\tilde{\mathbf{a}}=k \mathbf{a}$, onde $k$ é uma constante escalar não nula. Como $[\mathbf{a}]_{\times} \mathrm{A}=[\tilde{\mathbf{a}}]_{\times} \tilde{\mathrm{A}}$, segue que $[\mathbf{a}]_{\times}(k \tilde{\mathrm{A}}-\mathbf{A})=0, k \tilde{\mathrm{A}}-\mathbf{A}=\mathbf{a v}^{\mathrm{T}}$ e $\tilde{\mathrm{A}}=k^{-1}\left(\mathbf{A}+\mathbf{a v}^{\mathrm{T}}\right)$, onde $\mathbf{v}$ é um vetor qualquer. Assim, dado $\mathrm{P}^{\prime}=[\mathrm{A} \mid \mathbf{a}]$, tem-se $\tilde{\mathrm{P}}^{\prime}=\left[k^{-1}\left(\mathrm{~A}+\mathbf{a v}^{\mathrm{T}}\right) \mid k \mathbf{a}\right]$.

Seja uma transformação projetiva dada pela matriz:

$$
\mathrm{H}=\left[\begin{array}{cc}
k^{-1} \mathrm{I} & \mathbf{0} \\
k^{-1} \mathbf{v}^{\mathrm{T}} & k
\end{array}\right],
$$

então $\mathrm{PH}=k^{-1}[\mathrm{I} \mid \mathbf{0}]=k^{-1} \tilde{\mathrm{P}}$ e $\mathrm{P}^{\prime} \mathrm{H}=[\mathrm{A} \mid \mathbf{a}] \mathrm{H}=\left[k^{-1}\left(\mathrm{~A}+\mathbf{a v}^{\mathrm{T}}\right) \mid k \mathbf{a}\right]$, e portanto os pares de câmeras $\left(\mathrm{P}, \mathrm{P}^{\prime}\right)$ e $\left(\tilde{\mathrm{P}}, \tilde{\mathrm{P}}^{\prime}\right)$ são relacionados projetivamente.

\subsubsection{Câmeras Canônicas da Matriz Fundamental}

Dados um vetor 3D qualquer $\mathbf{v}$ e um escalar não nulo $\lambda$, a forma geral do par de matrizes de câmera canônicas correspondente à matriz fundamental $\mathrm{F}$ é:

\footnotetext{
${ }^{1}$ Pode-se obter os dois pares canônicos aplicando transformações projetivas a cada par de câmeras conforme necessário.
} 


$$
\mathrm{P}=[\mathrm{I} \mid \mathbf{0}] \quad \mathrm{P}^{\prime}=\left[\left[\mathbf{e}^{\prime}\right]_{\times} \mathrm{F}+\mathbf{e}^{\prime} \mathbf{v}^{\mathrm{T}} \mid \lambda \mathbf{e}^{\prime}\right]
$$

Seja F uma matriz fundamental e S uma matriz anti-simétrica qualquer. Considerando o epipolo $\mathbf{e}^{\prime}$ tal que $\mathbf{e}^{\prime} F=0$, e o par de matrizes de câmera $P=[\mathrm{I} \mid \mathbf{0}]$ e $\mathrm{P}^{\prime}=\left[\mathrm{SF} \mid \mathbf{e}^{\prime}\right]$, então $\mathrm{F}$ é a matriz fundamental correspondente ao par $\left(\mathrm{P}, \mathrm{P}^{\prime}\right)$. A condição de correspondência $\mathbf{x}^{\prime \mathrm{T}} \mathrm{F} \mathbf{x}=0$ equivale à condição da matriz $\mathrm{P}^{\prime \mathrm{T}} \mathrm{FP}$ ser anti-simétrica, uma vez que para um ponto $3 \mathrm{D}$ qualquer $\mathbf{X}$, tem-se $\mathbf{x}=\mathrm{PX}$, $\mathbf{x}^{\prime}=\mathrm{P}^{\prime} \mathbf{X}$, e $\mathbf{x}^{\prime \mathrm{T}} \mathrm{F} \mathbf{x}=\left(\mathrm{P}^{\prime} \mathbf{X}\right)^{\mathrm{T}} \mathrm{F}(\mathrm{PX})=\mathbf{X}^{\mathrm{T}}\left(\mathrm{P}^{\prime \mathrm{T}} \mathrm{FP}\right) \mathbf{X}=0$. Aplicando este resultado às matrizes $\mathrm{P}=[\mathrm{I} \mid \mathbf{0}]$ e $\mathrm{P}^{\prime}=\left[\mathrm{SF} \mid \mathbf{e}^{\prime}\right]$, obtém-se a seguinte matriz anti-simétrica:

$$
\left[\mathrm{SF} \mid \mathbf{e}^{\prime}\right] \mathrm{F}[\mathrm{I} \mid \mathbf{0}]=\left[\begin{array}{cc}
\mathrm{F}^{\mathrm{T}} \mathrm{S}^{\mathrm{T}} \mathrm{F} & \mathbf{0} \\
\mathbf{e}^{\prime \mathrm{T}} \mathrm{F} & 0
\end{array}\right]=\left[\begin{array}{cc}
\mathrm{F}^{\mathrm{T}} \mathrm{S}^{\mathrm{T}} \mathrm{F} & \mathbf{0} \\
\mathbf{0}^{\mathrm{T}} & 0
\end{array}\right]
$$

Escrevendo a matriz anti-simétrica $\mathbf{S}=[\mathbf{s}]_{\times}$em termos de seu vetor-nulo, para $\mathbf{s}^{\mathrm{T}} \mathbf{e}^{\prime} \neq 0$ a matriz $\mathrm{P}^{\prime}=\left[[\mathbf{s}]_{\times} \mathrm{F} \mid \mathbf{e}^{\prime}\right]$ tem posto 3 e representa uma matriz de câmera válida. Como $\mathbf{e}^{{ }^{\mathrm{T}} \mathrm{F}}=0$, o espaço-coluna de $\mathrm{F}$ é perpendicular a $\mathbf{e}^{\prime}$. Se $\mathbf{s}^{\mathrm{T}} \mathbf{e}^{\prime} \neq 0, \mathbf{s}$ não é perpendicular a $\mathbf{e}^{\prime}$ e não pertence ao espaço-coluna de F. Assim, o produto $[\mathbf{s}]_{\times} \mathbf{F}$ representa o plano perpendicular a $\mathbf{s}$, e tem posto 2 . Como $\mathbf{e}^{\prime}$ não é perpendicular a $\mathbf{s}$, não pertence àquele plano, de forma que $\left.\left[[\mathbf{s}]_{\times}\right] \mathbf{F} \mid \mathbf{e}^{\prime}\right]$ tem posto 3. Conforme Luong e Viéville (LUONG; VIÉVILLE, 1996), pode-se escolher $\mathbf{S}=\left[\mathbf{e}^{\prime}\right]_{\times}$, pois $\mathbf{e}^{\mathbf{T}^{\mathrm{T}}} \mathbf{e}^{\prime} \neq 0$. Portanto, as matrizes de câmera correspondentes a uma matriz fundamental $f$ podem ser escolher como $P=[\mathrm{I} \mid \mathbf{0}]$ e $\mathrm{P}^{\prime}=\left[\left[\mathbf{e}^{\prime}\right]_{\times} F \mid \mathbf{e}^{\prime}\right]$. Neste caso, a submatriz $3 \times 3$ da esquerda de matriz $\mathrm{P}^{\prime}$ tem posto 2 , de forma que representa uma câmera com centro em $\pi_{\infty}$. Como o par de câmeras $\mathrm{P}=[\mathrm{I} \mid \mathbf{0}]$ e $\mathrm{P}^{\prime}=\left[\left[\mathbf{e}^{\prime}\right]_{\times} \mathrm{F}+\mathbf{e}^{\prime} \mathbf{v}^{\mathrm{T}} \mid \lambda \mathbf{e}^{\prime}\right]$ equivale ao par $\mathrm{P}=[\mathrm{I} \mid \mathbf{0}]$ e $\mathrm{P}^{\prime}=[\mathrm{A} \mid \mathbf{a}]$, a equação (4.16) representa a forma geral das matrizes de projeção de câmera canônicas.

\subsubsection{Ambigüidade Reconstrutiva}

A reconstrução da posição absoluta ou da orientação de uma cena a partir de um par de imagens geralmente não é possível sem o prévio conhecimento de alguma informação a respeito de sua posição em relação a um sistema de coordenadas 3D, independentemente de qualquer conhecimento sobre os parâmetros intrínsecos das câmeras e da posição relativa entre elas. Em outras palavras, a cena pode ser determinada a menos de uma transformação Euclidiana (rotação e translação) em relação ao sistema de coordenadas global.

Outro fato importante é que também é impossível determinar o fator de escala 
global da cena apenas a partir de um par de imagens. Novamente é necessário que hajam informações complementares, sem as quais a cena é determinada a menos de uma transformação similar (rotação, translação e escalonamento).

Sejam $\mathbf{X}_{i}$ um conjunto de pontos 3D, e P e $\mathrm{P}^{\prime}$ um par de câmeras que projetam os pontos $3 \mathrm{D} \mathbf{X}_{i}$ em pontos $\mathbf{x}_{i}$ e $\mathbf{x}_{i}^{\prime}$ de duas imagem. Os pontos $\mathbf{X}_{i}$ e o par de câmeras $\left(\mathrm{P}, \mathrm{P}^{\prime}\right)$ constituem a reconstrução da cena a partir da correspondência entre imagens. Seja ainda:

$$
\mathrm{H}_{\mathrm{S}}=\left[\begin{array}{cc}
\mathrm{R} & \mathbf{t} \\
\mathbf{0}^{\mathrm{T}} & \lambda
\end{array}\right],
$$

uma transformação similar qualquer: $\mathrm{R}$ é uma rotação, $\mathbf{t}$ é uma translação e $\lambda^{-1}$ é um escalonamento global. Substituindo cada ponto $\mathbf{X}_{i}$ por $\mathrm{H}_{\mathrm{S}} \mathbf{X}_{i}$, e as câmeras $\left(\mathrm{P}, \mathrm{P}^{\prime}\right)$ por $\left(\mathrm{PH}_{\mathrm{S}}{ }^{-1}, \mathrm{P}^{\prime} \mathrm{H}_{\mathrm{S}}{ }^{-1}\right)$ não altera os pontos $\mathbf{x}_{i}$ e $\mathbf{x}_{i}^{\prime}$ das imagens, pois $\mathrm{PX}_{i}=\left(\mathrm{PH}_{\mathrm{S}}{ }^{-1}\right)\left(\mathrm{H}_{\mathrm{S}} \mathbf{X}_{i}\right)$. Adicionalmente, se $\mathrm{P}$ for decomposta como $\mathrm{P}=\mathrm{K}\left[\mathrm{R}_{\mathrm{P}} \mid \mathbf{t}_{\mathrm{P}}\right]$, tem-se:

$$
\mathrm{PH}_{\mathrm{S}}{ }^{-1}=\mathrm{K}\left[\mathrm{R}_{\mathrm{P}} \mathrm{R}^{-1} \mid \mathbf{t}^{\prime}\right]
$$

para algum t' cujo cálculo exato é irrelevante. Este resultado mostra que multiplicando-se a matriz de câmera $\mathrm{P}$ por $\mathrm{H}_{\mathrm{S}}{ }^{-1}$ a matriz de calibração permanece inalterada, e portanto a ambigüidade reconstrutiva ocorre mesmo no caso de câmeras calibradas. Em (LONGUETT-HIGGINS, 1981), demonstra-se que esta é a única ambigüidade reconstrutiva no caso de câmeras calibradas. Assim, para câmeras calibradas a reconstrução é possivel a menos de uma transformação similar, conforme ilustrado na Figura 4.7(a). Se as câmeras são calibradas, então qualquer reconstrução deve preservar o ângulo medido entre raios das imagens. Uma transformação similar da estrutura e das câmeras não altera tais ângulos. Os ângulo entre os raios e a reta base (epipolos) também permanecem inalterados. Se as câmeras não são calibradas, a reconstrução deve preservar os pontos das imagens (a intersecção dos raios com os planos de imagem). Uma transformação projetiva da estrutura e das câmeras não altera os pontos medidos, apesar do ângulo entre os raios ser alterado. Os epipolos também permanecem inalterados (intersecção da reta base com os planos de imagem), conforme ilustrado na Figura 4.7(b).

Ambigüidade Projetiva. Se tanto a calibração de cada câmera como a posição absoluta de uma delas forem desconhecidas, a ambigüidade reconstrutiva é expressa em termos de uma transformação projetiva arbitrária. Como visto na 


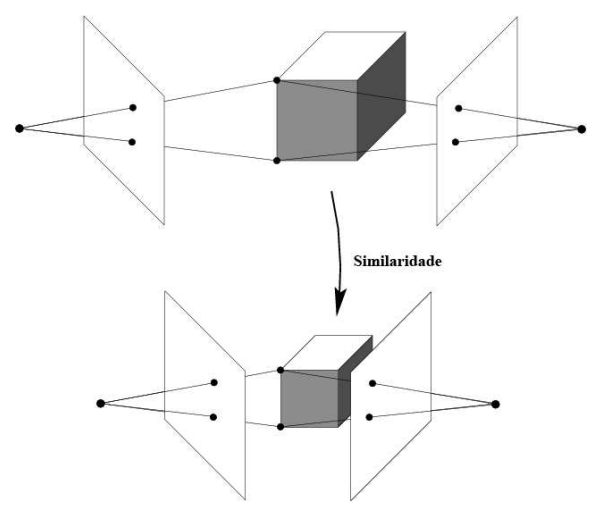

(a)

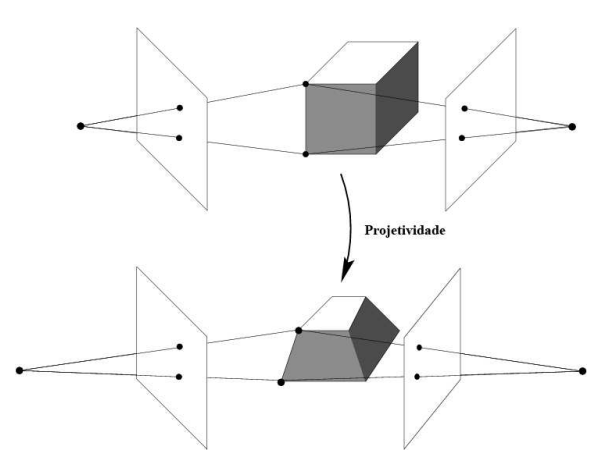

(b)

Figura 4.7: Ambigüidade Reconstrutiva. (a) Transformação similar. (b) Transformação projetiva.

Seção 4.4.2, se H é uma matriz $4 \times 4$ inversível representando uma transformação projetiva de $\mathbf{P}^{\mathbf{3}}$, então a substituição de pontos $\mathbf{X}_{i}$ por $\mathrm{H} \mathbf{X}_{i}$ e das matrizes $\left(\mathrm{P}, \mathrm{P}^{\prime}\right)$ por $\left(\mathrm{PH}^{-1}, \mathrm{P}^{\prime} \mathrm{H}^{-1}\right)$ não altera os pontos da imagem. Assim, para câmeras nãocalibradas a reconstrução é possível a menos de uma transformação projetiva.

Outras formas de ambigüidade surgem para alguns movimentos especiais de câmera ou para informações parciais sobre as mesmas, em particular se duas câmeras estão relacionadas por um movimento de translação pura, sem alteração da calibração, então a reconstrução é possível a menos de uma transformação afim, e se duas câmeras são calibradas a menos de suas distâncias focais, então a reconstrução é possível a menos de uma transformação similar.

Terminologia. Além da reconstrução real das correspondências $\mathbf{x}_{i} \leftrightarrow \mathbf{x}_{i}^{\prime}$, formada pelos verdadeiros pontos $\tilde{\mathbf{X}}_{i}$ e pelas verdadeiras câmeras $\left(\tilde{\mathrm{P}}, \tilde{\mathrm{P}}^{\prime}\right)$ que geraram o par de imagens, o conjunto $\mathbf{X}_{i}$ de pontos reconstruídos a partir de um par de câmeras relativo à matriz fundamental da correspondência entre as imagens pode diferir da reconstrução real por uma transformação pertencente a um determinado grupo dentre e hierarquia de transformações projetivas. Assim, tem-se reconstrução projetiva, reconstrução afim e reconstrução similar. O termo reconstrução métrica refere-se normalmente à reconstrução similar, indicando que propriedades métricas como ângulos entre retas e razões entre distâncias podem ser medidos a partir da reconstrução. Além deste, o termo reconstrução Euclidiana também é utilizado para indicar a reconstrução similar. 


\subsubsection{Teorema da Reconstrução Projetiva}

Definido em (HARTLEY; ZISSERMAN, 2000), o teorema da reconstrução projetiva a partir de duas câmeras não-calibradas diz que se um conjunto de pontos correspondentes em duas imagens determina exatamente a matriz fundamental, então a cena e as câmeras podem ser reconstruídas a partir destas correspondências, $e$ quaisquer duas reconstruções assim obtidas são projetivamente equivalentes. Este resultado não é valido para os pontos pertencentes à reta base, que não podem ser reconstruídos mesmo quando as matrizes de câmera originais são conhecidas.

Formalmente, dado o conjunto de correspondências entre duas imagens $\mathbf{x}_{i} \leftrightarrow$ $\mathbf{x}_{i}^{\prime}$ tais que a matriz fundamental $\mathbf{F}$ fica exatamente determinada pela condição $\mathbf{x}_{i}^{\prime \mathrm{T}} \mathbf{F} \mathbf{x}_{i}$ para todo $i$, e sejam $\left(\mathrm{P}_{1}, \mathrm{P}_{1}^{\prime},\left\{\mathbf{X}_{1 i}\right\}\right)$ e $\left(\mathrm{P}_{2}, \mathrm{P}_{2}^{\prime},\left\{\mathbf{X}_{2 i}\right\}\right)$ duas reconstruções destas correspondências $\mathbf{x}_{i} \leftrightarrow \mathbf{x}_{i}^{\prime}$, então uma matriz não-singular $\mathrm{H}$ tal que $\mathrm{P}_{2}=$ $\mathrm{P}_{1} \mathrm{H}^{-1}, \mathrm{P}_{2}^{\prime}=\mathrm{P}_{1}^{\prime} \mathrm{H}^{-1}$ e $\mathbf{X}_{2 i}=\mathrm{HX}_{1 i}$ ) para todo $i$, exceto aqueles em que $\mathbf{F}_{i}=$ $\mathbf{x}_{i}^{\prime \mathrm{T}} \mathrm{F}=0$.

Como ambos os pares de matrizes de câmera $\left(\mathrm{P}_{1}, \mathrm{P}_{1}^{\prime}\right)$ e $\left(\mathrm{P}_{2}, \mathrm{P}_{2}^{\prime}\right)$ correspondem à matriz fundamental $\mathrm{F}$, então existe uma transformação projetiva $\mathrm{H}$ tal que $\mathrm{P}_{1}=\mathrm{P}_{2} \mathrm{H}$ e $\mathrm{P}_{1}^{\prime}=\mathrm{P}_{2}^{\prime} \mathrm{H}$, conforme descrito na Seção 4.4.2. Observa-se que $\mathrm{P}_{2}\left(\mathrm{HX}_{1 i}\right)=$ $\mathrm{P}_{1} \mathrm{H}^{-1} \mathrm{H} \mathbf{X}_{1 i}=\mathrm{P}_{1} \mathbf{X}_{1 i}=\mathbf{x}_{i}$. Por outro lado, $\mathrm{P}_{2} \mathbf{X}_{2 i}=\mathbf{x}_{i}$, e $\mathrm{P}_{2}\left(\mathrm{H}_{1 i}\right)=\mathrm{P}_{2} \mathbf{X}_{2 i}$. Assim, tanto $\mathrm{HX}_{1 i}$ como $\mathbf{X}_{2 i}$ estão sobre o mesmo raio que passa pelo centro de câmera de $\mathrm{P}_{2}$. Analogamente, estes dois pontos estão sobre o raio que passa pelo centro de câmera de $\mathrm{P}_{2}^{\prime}$. Nestas condições, os pontos $\mathrm{HX}_{1 i}$ e $\mathbf{X}_{2 i}$ só são distintos se estiverem sobre a reta que passa pelos dois centros de câmera. Então $\mathbf{X}_{2 i}=\mathrm{HX}_{1 i}$, exceto quando os pontos $\mathbf{x}_{i}$ e $\mathbf{x}_{i}^{\prime}$ coincidem com os epipolos da imagem, e $\mathbf{F} \mathbf{x}_{i}=\mathbf{x}_{i}^{\prime \mathrm{T}} \mathrm{F}=0$.

Este resultado implica que é possível determinar a reconstrução projetiva de uma cena baseado apenas na correspondência entre imagens, sem qualquer informação sobre a calibração ou a posição relativa das duas câmeras.

Supondo que a reconstrução Euclidiana real seja $\left(\mathrm{P}_{\mathrm{E}}, \mathrm{P}_{\mathrm{E}}^{\prime},\left\{\mathbf{X}_{\mathrm{E} i}\right\}\right)$ e a reconstrução projetiva seja $\left(\mathrm{P}, \mathrm{P}^{\prime},\left\{\mathbf{X}_{i}\right\}\right)$, então estas duas reconstruções relacionam-se por uma matriz não-singular $\mathrm{H}$ tal que:

$$
\mathrm{P}_{\mathrm{E}}=\mathrm{PH}^{-1}, \mathrm{P}_{\mathrm{E}}^{\prime}=\mathrm{P}^{\prime} \mathrm{H}^{-1} \text { e } \mathbf{X}_{\mathrm{E} i}=\mathrm{HX}_{i}
$$

onde H é uma homografia representada por uma matriz $4 \times 4$ idêntica para todos os pontos. 


\section{Cálculo da Matriz Fundamental}

O cálculo da matriz fundamental é realizado a partir da condição de correspondência, definida na Seção 4.2.3. A partir de um conjunto de pares de pontos correspondentes $\mathbf{x} \leftrightarrow \mathbf{x}^{\prime}$ determina-se um sistema de equações homogêneo, cuja solução é a base para todos algorítmos apresentados a seguir.

Os conceitos apresentados neste capítulo foram extraídos de (HARTLEY; ZISSERMAN, 2000), (FAUGERAS, 1993), (ULLMAN, 1979), e (XU; ZHANG, 1996). A notação adotada é a de (HARTLEY; ZISSERMAN, 2000).

\subsection{Cálculo Básico}

A matriz fundamental é definida pela condição de correspondência, representada pela equação (4.9): $\mathbf{x}^{\prime \mathrm{T}} \mathrm{F} \mathbf{x}=0$, para qualquer par de pontos correspondentes $\mathbf{x} \leftrightarrow \mathbf{x}^{\prime}$ em duas imagens. Dado um conjunto suficientemente grande de pontos correspondentes $\mathbf{x} \leftrightarrow \mathbf{x}^{\prime}$ (ao menos 7), a equação (4.9) pode ser usada para calcular a matriz F. Em particular, para $\mathbf{x}=(x, y, 1)^{\mathrm{T}}$ e $\mathbf{x}^{\prime}=\left(x^{\prime}, y^{\prime}, 1\right)^{\mathrm{T}}$, cada par de pontos correspondentes determina uma equação linear em função dos elementos de $\mathrm{F}=\left[f_{i j}\right]$ :

$$
x^{\prime} x f_{11}+x^{\prime} y f_{12}+x^{\prime} f_{13}+y^{\prime} x f_{21}+y^{\prime} y f_{22}+y^{\prime} f_{23}+x f_{31}+y f_{32}+f_{33}=0 .
$$

Denominando $\mathbf{f}$ o vetor de nove elementos composto pelos coeficientes de $\mathrm{F}$, a equação (5.1) pode ser expressa como $\left(x^{\prime} x, x^{\prime} y, x^{\prime}, y^{\prime} x, y^{\prime} y, y^{\prime}, x, y, 1\right)^{\mathrm{T}} \mathbf{f}$. A partir de um conjunto de $n$ pares de pontos correspondentes obtém-se um sistema de equações lineares: 


$$
\text { Af }=\left[\begin{array}{ccccccccc}
x_{1}^{\prime} x_{1} & x_{1}^{\prime} y_{1} & x_{1}^{\prime} & y_{1}^{\prime} x_{1} & y_{1}^{\prime} y_{1} & y_{1}^{\prime} & x_{1} & y_{1} & 1 \\
\vdots & \vdots & \vdots & \vdots & \vdots & \vdots & \vdots & \vdots & \vdots \\
x_{n}^{\prime} x_{n} & x_{n}^{\prime} y_{n} & x_{n}^{\prime} & y_{n}^{\prime} x_{n} & y_{n}^{\prime} y_{n} & y_{n}^{\prime} & x_{n} & y_{n} & 1
\end{array}\right] \mathbf{f}=\mathbf{0}
$$

A matriz A da equação (5.2) representa um sistema de equações lineares homogêneo, e f pode ser determinado a menos de um fator de escala. Para que exista um solução, A deve ter posto no máximo igual a oito, caso em que a solução é única, determinada pelo gerador do espaço nulo à direita de A. Se os pontos correspondentes são inexatos, devido a ruídos em suas coordenadas, A pode ter posto igual a nove, caso em que a solução é determinada por mínimos-quadrados. Neste caso, $\mathbf{f}$ corresponde ao vetor singular referente ao menor valor singular da $\mathrm{SVD}^{1}$ de $A=U V^{\mathrm{T}}$, que minimiza $\|\mathrm{Af}\|$, sob a condição de $\|\mathbf{f}\|=1$. Esta minimização representa apenas a solução de melhor ajuste ao conjunto de pontos, não havendo relação deste critério com medidas tomadas nas imagens, que portanto não tem significado físico.

A matriz fundamental $\mathrm{F}$ é singular, de posto 2. Esta propriedade é denominada restrição de singularidade. A solução do sistema de equações (5.2) não necessariamente tem posto 2 , e esta condição deve ser garantida corrigindo-se a matriz F obtida da SVD de A. Assim, substitui-se a matriz F pela matriz $\mathrm{F}^{\prime}$ que minimiza a norma de Frobenius ${ }^{2}\left\|F-F^{\prime}\right\|_{F}^{2}$, sob a condição $\operatorname{de~} \operatorname{det}\left(F^{\prime}\right)=0$. Seja $\mathrm{F}=\mathrm{UDV}^{\mathrm{T}}$ a $\operatorname{SVD}$ de $\mathrm{F}$, onde $\mathrm{D}=\operatorname{diag}(r, s, t)$ satisfaz $r \geq s \geq t$. Então $\mathrm{F}^{\prime}=\operatorname{Udiag}(r, s, 0) \mathrm{V}^{\mathrm{T}}$ minimiza a norma de Frobenius $\left\|\mathrm{F}-\mathrm{F}^{\prime}\right\|_{\mathrm{F}}^{2} \operatorname{edet}\left(\mathrm{F}^{\prime}\right)=0$.

\subsection{Algoritmo de 8 Pontos Correspondentes}

A solução do sistema de equações A por SVD e posterior aplicação da restrição de singularidade descritos anteriormente constitui o algoritmo básico para cálculo da matriz fundamental, denominado Algoritmo de 8 Pontos Correspondentes. Este algoritmo é a forma mais simples de cálculo de $\mathrm{F}$, e pode ser formulado em dois passos:

1. Solução Linear. Obtém-se F do vetor $\mathbf{f}$ correspondente ao menor valor singular de A, definida em (5.2).

2. Restrição de Singularidade. Substitui-se $\mathrm{F}=\operatorname{Udiag}(r, s, t) \mathrm{V}^{\mathrm{T}}, r \geq s \geq t$, por $\mathrm{F}^{\prime}=\operatorname{Udiag}(r, s, 0) \mathrm{V}^{\mathrm{T}}$, a matriz singular de posto 2 que melhor aproxima

\footnotetext{
${ }^{1}$ Decomposição em valores singulares, vide detalhes no Apêndice A, Seção A.4

${ }^{2}$ Vide Apêndice A, Seção A.5
} 
F segundo a norma de Frobenius.

\subsection{Algoritmo de 7 Pontos Correspondentes}

Se o sistema de equações A definido em (5.2) tem posto 8, existe uma única solução f determinada a menos de um fator de escala. No caso de a matriz ter posto 7, é possível determinar um conjunto de soluções para a matriz fundamental utilizando-se a restrição de singularidade (já que $\mathrm{F}$ tem 7 graus de liberdade). O caso mais importante é aquele em que são conhecidas apenas 7 pares de pontos correspondentes, gerando uma matriz A $7 \times 9$, geralmente de posto 7 .

Neste caso, a solução do sistema de equações Af $=\mathbf{0}$ é um espaço bidimensional da forma $\alpha \mathrm{F}_{1}+(1-\alpha) \mathrm{F}_{2}$, onde $\alpha$ é uma variável escalar. As matrizes $\mathrm{F}_{1}$ e $F_{2}$ são obtidas dos geradores $\mathbf{f}_{1}$ e $\mathbf{f}_{2}$ do espaço nulo à direita de $\mathrm{A}$. A restrição $\operatorname{det}(\mathrm{F})=0$ pode ser escrita como $\operatorname{det}\left(\alpha \mathrm{F}_{1}+(1-\alpha) \mathrm{F}_{2}\right)=0$, o que gera uma equação polinomial de $3^{\circ}$ grau na variável $\alpha$. Esta equação tem uma ou três soluções reais em $\alpha$ - as soluções complexas são descartadas (HARTLEY, 1994). Substituindo-se estes valores de $\alpha$ na equação $\mathrm{F}=\alpha \mathrm{F}_{1}+(1-\alpha) \mathrm{F}_{2}$, obtém-se uma ou três soluções possíveis para a matriz fundamental.

\subsection{Algoritmo de 8 Pontos Correspondentes Nor- malizado}

O Algoritmo de 8 Pontos Correspondentes descrito na Seção 5.2 é o método mais simples de cálculo da matriz fundamental, envolvendo apenas a solução por mínimos-quadrados de um sistema de equações. O algoritmo original foi formulado em (LONGUETT-HIGGINS, 1981), e a chave para o seu bom desempenho é a normalização adequada e sistemática dos pontos correspondentes (Apêndice A, Seção A.6). No caso do Algoritmo de 8 Pontos Correspondentes, uma transformação de translação e escalonamento aplicada sobre os pontos das imagens antes da construção do sistema de equações $\mathbf{A f}=\mathbf{0}$ garante melhoria do seu condicionamento e da estabilidade do resultado sem aumento significativo de complexidade do algoritmo.

A normalização consiste na translação e escalonamento de cada imagem de forma que o centróide dos pontos de referência seja deslocado para a origem do sistema de coordenadas de imagem e a distância quadrática média destes pontos em relação à origem seja $\sqrt{2}$. 
Dados $n \geq 8$ pares de pontos correspondentes em duas imagens, $\left\{\mathbf{x}_{i} \leftrightarrow \mathbf{x}_{i}^{\prime}\right\}$, a matriz fundamental $\mathrm{F}$ tal que $\mathbf{x}_{i}^{\prime \mathrm{T}} \mathrm{F} \mathbf{x}_{i}=0$ é determinada pelo Algoritmo de 8 Pontos Correspondentes Normalizado segundo os seguintes passos:

1. Normalização. Transformam-se as coordenadas de imagem conforme $\hat{\mathbf{x}}_{i}=$ $\mathrm{Tx}_{i}$ e $\hat{\mathbf{x}}_{i}^{\prime}=\mathrm{T}^{\prime} \mathbf{x}_{i}^{\prime}$, onde $\mathrm{T}$ e T $\mathrm{T}^{\prime}$ são transformações de normalização compostas de translação e escalonamento (Apêndice A, Seção A.6).

2. Cálculo da Matriz Fundamental. Determina-se a matriz fundamental $\hat{\mathrm{F}}^{\prime}$ a partir dos pontos correspondentes $\left\{\hat{\mathbf{x}}_{i} \leftrightarrow \hat{\mathbf{x}}_{i}^{\prime}\right\}$ :

(a) Solução Linear. Determina-se $\hat{F}$ a partir do vetor singular correspondente ao menor valor singular de A, composta a partir das correspondências $\left\{\hat{\mathbf{x}}_{i} \leftrightarrow \hat{\mathbf{x}}_{i}^{\prime}\right\}$, conforme a equação (5.2).

(b) Restrição de Singularidade. Determina-se $\hat{F}^{\prime}$ tal que $\operatorname{det}\left(\hat{\mathrm{F}}^{\prime}\right)=0$, dada por $\hat{\mathrm{F}}^{\prime}=\hat{\mathrm{U}} \operatorname{diag}(\hat{r}, \hat{s}, 0) \hat{\mathrm{V}}^{\mathrm{T}}$, a partir da $\operatorname{SVD}$ de $\hat{\mathrm{F}}=\hat{\mathrm{U}} \operatorname{diag}(\hat{r}, \hat{s}, \hat{t}) \hat{\mathrm{V}}^{\mathrm{T}}$, $\hat{r} \geq \hat{s} \geq \hat{t}$. (A restrição de singularidade deve ser garantida antes da normalização reversa (HARTLEY, 1997a).)

3. Normalização Reversa. Determina-se $F=\mathrm{T}^{\prime} \mathrm{T} \hat{\mathrm{F}}^{\prime} \mathrm{T}$, a matriz fundamental correspondente ao conjunto original $\left\{\mathbf{x}_{i} \leftrightarrow \mathbf{x}_{i}^{\prime}\right\}$.

\subsection{Distância Epipolar e Erro Residual}

A distância epipolar é a distância entre um ponto da imagem e a reta epipolar correspondente, determinada a partir da matriz fundamental. Dados a matriz fundamental $\mathrm{F}$ da correspondência entre duas imagens, um par de pontos correspondentes $\mathbf{x} \leftrightarrow \mathbf{x}^{\prime}$ destas imagens, e as respectivas retas epipolares $\mathbf{l}=\mathrm{F}^{\mathrm{T}} \mathbf{x}^{\prime}$ e $\mathbf{l}^{\prime}=\mathrm{Fx}$, tem-se:

$$
\begin{aligned}
& \mathrm{d}_{\text {epipolar }}=\mathrm{d}(\mathbf{x}, \mathbf{l})=\mathrm{d}\left(\mathbf{x}, \mathrm{F}^{\mathrm{T}} \mathbf{x}^{\prime}\right)=\frac{\left|\mathbf{x}^{\mathrm{T}} \mathrm{F}^{\mathrm{T}} \mathbf{x}^{\prime}\right|}{\sqrt{\left(\mathbf{f}_{1}^{\mathrm{T}} \mathbf{x}\right)^{2}+\left(\mathbf{f}_{2}^{\mathrm{T}} \mathbf{x}\right)^{2}}}, \\
& \mathrm{~d}_{\text {epipolar }}^{\prime}=\mathrm{d}\left(\mathbf{x}^{\prime}, \mathbf{l}^{\prime}\right)=\mathrm{d}\left(\mathbf{x}^{\prime}, \mathbf{F} \mathbf{x}\right)=\frac{\left|\mathbf{x}^{\prime^{\mathrm{T}} \mathbf{x} \mid}\right|}{\sqrt{\left(\mathbf{f}^{1} \mathbf{x}^{\prime}\right)^{2}+\left(\mathbf{f}^{\mathrm{T}} \mathbf{x}^{\prime}\right)^{2}}},
\end{aligned}
$$

onde o vetor $\mathbf{f}_{j}$ representa a $j$-ésima coluna e o vetor $\mathbf{f}^{j^{\mathrm{T}}}$ representa a $j$-ésima linha da matriz fundamental $\mathrm{F}$.

O erro residual de um conjunto de $N$ correspondências $\left\{\mathbf{x}_{i} \leftrightarrow \mathbf{x}_{i}^{\prime}\right\}$ é a média das distâncias epipolares quadráticas, tomadas simetricamente na duas imagens: 


$$
\mathrm{e}_{\text {residual }}=\frac{1}{N} \sum_{i}\left(\mathrm{~d}\left(\mathbf{x}_{i}^{\prime}, \mathbf{l}_{i}^{\prime}\right)^{2}+\mathrm{d}\left(\mathbf{x}_{i}, \mathbf{l}_{i}\right)^{2}\right)
$$

Alternativamente, substituindo as equações (5.3) na equação (5.4), tem-se:

$$
\mathrm{e}_{\text {residual }}=\frac{1}{N} \sum_{i}\left(\mathbf{x}^{\prime \mathrm{T}} \mathrm{F} \mathbf{x}\right)^{2}\left(\frac{1}{\left(\mathbf{f}^{1^{\mathrm{T}}} \mathbf{x}^{\prime}\right)^{2}+\left(\mathbf{f}^{2 \mathrm{~T}} \mathbf{x}^{\prime}\right)^{2}}+\frac{1}{\left(\mathbf{f}_{1}^{\mathrm{T}} \mathbf{x}\right)^{2}+\left(\mathbf{f}_{2}{ }^{\mathrm{T}} \mathbf{x}\right)^{2}}\right)
$$

Observa-se que os algoritmos descritos nas seções anteriores não operam sobre o erro residual, ou quaisquer outras medidas tomadas nas imagens.

\subsection{Busca Exaustiva}

Para um conjunto de $N$ pares de pontos correspondentes $\left\{\mathbf{x}_{i} \leftrightarrow \mathbf{x}_{i}^{\prime}\right\}$, o Algoritmo de 8 Pontos Correspondentes Normalizado fornece diretamente a matriz fundamental que melhor se ajusta a este conjunto de pontos. Devido à presença de ruídos de medida, a matriz fundamental obtida a partir do Algoritmo de 7 Pontos Correspondentes, utilizando sete pares de pontos escolhidos aleatoriamente dentro do conjunto de $N$ pares de pontos correspondentes, pode não ser a melhor solução em relação aos demais pontos do conjunto. Isto pode ser verificado avaliando-se $\mathbf{x}_{i}^{\prime \mathrm{T}} \mathrm{Fx}_{i}$ em todo o conjunto de $N$ pares de pontos correspondentes. A forma mais simples de se determinar a matriz fundamental ótima a partir do Algoritmo de 7 Pontos Correspondentes é realizar uma busca exaustiva dentro do conjunto de pontos correspondentes, sobre todas as combinações de sete pares de pontos possíveis. A matriz fundamental ótima é aquela que minimiza um critério de escolha, o valor médio da relação $\mathbf{x}_{i}^{\prime \mathrm{T}} \mathrm{F} \mathbf{x}_{i}$ no conjunto de $N$ pares de pontos correspondentes. Outro critério de avaliação da matriz fundamental é o erro residual, tomado a partir de medições sobre as imagens, e portanto com significado físico. Neste caso, a matriz fundamental ótima é aquela que fornece o menor erro residual no conjunto de $N$ pares de pontos correspondentes. Este método de determinação da matriz fundamental que melhor se ajusta a um conjunto de pontos, derivado do Algoritmo de 7 Pontos Correspondentes, tem a desvantagem de ser computacionalmente custoso, envolvendo $K=N ! /(7 !(N-7) !)$ iterações. 


\subsection{Solução Linear Iterativa (Ponderada)}

A solução do sistema de equações Af $=\mathbf{0}$ a partir da SVD de A equivale a determinar $\min _{\mathbf{f}}\|\mathbf{A f}\|$, dado que $\|\mathbf{f}\|=1$. Assim, dados a matriz fundamental $\mathrm{F}$ da correspondência entre duas imagens, um par de pontos correspondentes $\mathbf{x} \leftrightarrow \mathbf{x}^{\prime}$ destas imagens, e as respectivas retas epipolares $\mathbf{l}=\mathrm{F}^{\mathrm{T}} \mathbf{x}^{\prime}$ e $\mathbf{l}^{\prime}=\mathbf{F} \mathbf{x}$, tem-se (vide Apêndice A, Seção A.6):

$$
\min _{\mathbf{f}}\|\mathrm{Af}\|=\min _{\mathrm{F}} \sum_{i}\left(\mathbf{x}_{i}^{\prime \mathrm{T}} \mathrm{F} \mathbf{x}\right)^{2}
$$

Para a segunda imagem, tem-se:

$$
\min _{\mathbf{f}}\|\mathrm{Af}\|=\min _{\mathrm{F}} \sum_{i}\left(\left(\mathbf{f}^{1^{\mathrm{T}}} \mathbf{x}^{\prime}\right)^{2}+\left(\mathbf{f}^{2^{\mathrm{T}}} \mathbf{x}^{\prime}\right)^{2}\right) \mathrm{d}\left(\mathbf{x}^{\prime}, \mathbf{l}^{\prime}\right)^{2}
$$

onde o vetor $\mathbf{f}^{j^{\mathrm{T}}}$ representa a $j$-ésima linha da matriz fundamental F. Analogamente, para a primeira imagem tem-se:

$$
\min _{\mathbf{f}}\|\mathbf{A f}\|=\min _{\mathbf{F}} \sum_{i}\left(\left(\mathbf{f}_{1}{ }^{\mathrm{T}} \mathbf{x}\right)^{2}+\left(\mathbf{f}_{2}{ }^{\mathrm{T}} \mathbf{x}\right)^{2}\right) \mathrm{d}(\mathbf{x}, \mathbf{l})^{2}
$$

onde o vetor $\mathbf{f}_{j}$ representa a $j$-ésima coluna da matriz fundamental $\mathrm{F}$.

A utilização do erro residual definido na equação (5.4) como critério de escolha da matriz fundamental equivale a determinar:

$$
\min _{\mathrm{F}} \sum_{i}\left(\mathrm{~d}\left(\mathbf{x}_{i}^{\prime}, \mathbf{l}_{i}^{\prime}\right)^{2}+\mathrm{d}\left(\mathbf{x}_{i}, \mathbf{l}_{i}\right)^{2}\right)
$$

Assim, pela equação (5.5) tem-se:

$$
\min _{\mathrm{F}} \sum_{i}\left(\mathrm{~d}\left(\mathbf{x}_{i}^{\prime}, \mathbf{l}_{i}^{\prime}\right)^{2}+\mathrm{d}\left(\mathbf{x}_{i}, \mathbf{l}_{i}\right)^{2}\right)=\min _{\mathrm{F}} \sum_{i} k_{i}^{2}\left(\mathbf{x}^{\mathbf{\prime}^{\mathrm{T}}} \mathbf{F} \mathbf{x}\right)^{2}
$$

onde:

$$
k_{i}^{2}=\frac{1}{\left(\mathbf{f}^{1^{\mathrm{T}}} \mathbf{x}^{\prime}\right)^{2}+\left(\mathbf{f}^{2^{\mathrm{T}}} \mathbf{x}^{\prime}\right)^{2}}+\frac{1}{\left(\mathbf{f}_{1}^{\mathrm{T}} \mathbf{x}\right)^{2}+\left(\mathbf{f}_{2}{ }^{\mathrm{T}} \mathbf{x}\right)^{2}}
$$

Denominando $\mathbf{k}$ o vetor formado pelos coeficientes $k_{i}$, pode-se escrever a relação acima como: 


$$
\min _{\mathrm{F}} \sum_{i} k_{i}^{2}\left(\mathbf{x}^{\prime \mathrm{T}} \mathrm{F} \mathbf{x}\right)^{2}=\min _{\mathrm{F}} \sum_{i}\left(k_{i}\left(\mathbf{x}^{\prime{ }^{\mathrm{T}}} \mathrm{F} \mathbf{x}\right)\right)\left(\left(\mathbf{x}^{\prime \mathrm{T}} \mathrm{F} \mathbf{x}\right) k_{i}\right)=\min _{\mathrm{F}}\|\mathbf{k A f}\|
$$

Desta forma, minimizar o erro residual equivale a determinar a matriz fundamental dos sistema de equações homogêneo $\mathbf{k A f}=\mathbf{0}$, uma versão ponderada do sistema original obtido do conjunto de pontos correspondentes $\left\{\mathbf{x}_{i} \leftrightarrow \mathbf{x}_{i}^{\prime}\right\}$. No entanto, os coeficientes $k_{i}$ dependem da solução f , e o sistema deixa de ser linear. Conforme proposto em (ZHANG, 1996), pode-se contornar o problema da dependência entre os coeficientes $k_{i}$ e a solução $\mathbf{f}$ aplicando-se um método iterativo linear. Assumindo a condição inicial $k_{i}^{0}=1$ para todo $i$, determina-se a matriz fundamental solucionando-se Af $=\mathbf{0}$ através do Algoritmo de 8 Pontos Correspondentes Normalizado. A partir desta solução inicial calculam-se novos coeficientes $k_{i}^{j}$ para cada iteração $j$, e determina-se a solução do sistema $k_{i}^{j} \mathrm{Af}^{j}=\mathbf{0}$ também através do Algoritmo de 8 Pontos Correspondentes Normalizado. Repete-se este procedimento até que seja atingido um valor de limiar para o erro residual ou o valor de $j$ ultrapasse o limite máximo de iterações. Inicialmente proposto em (ZHANG, 1996), este método de determinação da matriz fundamental minimizando o erro residual não leva em conta a restrição de singularidade da matriz fundamental, e portanto não apresenta melhora significativa em relação à solução linear original (Algoritmo de 8 Pontos Correspondentes Normalizado). 


\section{Incerteza da Matriz Fundamental}

O cálculo da matriz fundamental a partir de pares de pontos correspondentes em duas imagens está sujeito a incertezas causadas por ruídos nas coordenadas destes pontos e pela presença de pares de pontos espúrios. A avaliação da matriz fundamental estimada pelos métodos apresentados no capítulo anterior requer a modelagem desta incerteza, dada em termos da covariância da matriz fundamental, conforme proposto em (ZHANG, 1996). Esta informação é importante não apenas na determinação da incerteza da matriz fundamental, mas também para as operações que derivam dela, como a recuperação das matrizes de câmera.

Considerando a forma vetorizada da matriz fundamental como um vetor aleatório $\mathbf{f} \in \mathbf{R}^{\mathbf{m}}$ cuja média é o valor desejado da matriz fundamental, cada estimativa pode ser considerada uma amostra de $\mathbf{f}$ e a incerteza é dada pela matriz de covariância deste vetor aleatório f. A dimensão $m$ do vetor $\mathbf{f}$ será entre 7 e 9, dependendo do método de determinação e da parametrização da matriz fundamental utilizados. De modo geral, para um vetor aleatório $\mathbf{y} \in \mathbf{R}^{\mathbf{p}}$, cuja média é $\mathrm{E}[\mathbf{y}]$, a covariância de $\mathbf{y}$ é dada pela matriz simétrica positiva-definida:

$$
\Lambda_{\mathbf{y}}=\mathrm{E}\left[(\mathbf{y}-\mathrm{E}[\mathbf{y}])(\mathbf{y}-\mathrm{E}[\mathbf{y}])^{\mathrm{T}}\right]
$$

\subsection{Método Estatístico}

Dado um conjunto de $N$ amostras $\mathbf{y}_{i}$ do vetor aleatório $\mathbf{y}$, para $N$ suficientemente grande (geralmente $N>30$ ), a média $\mathrm{E}[\mathbf{y}]$ pode ser aproximada pela média das amostras, de forma que:

$$
\mathrm{E}[\mathbf{y}] \approx \mathrm{E}_{N}\left[\mathbf{y}_{i}\right]=\frac{1}{N} \sum_{i=1}^{N} \mathbf{y}_{i}
$$

Assim, a covariância $\Lambda_{\mathbf{y}}$ pode ser aproximada por: 


$$
\Lambda_{\mathbf{y}} \approx \frac{1}{N-1} \sum_{i=1}^{N}\left[\left(\mathbf{y}_{i}-\mathrm{E}_{N}\left[\mathbf{y}_{i}\right]\right)\left(\mathbf{y}_{i}-\mathrm{E}_{N}\left[\mathbf{y}_{i}\right]\right)^{\mathrm{T}}\right]
$$

\subsection{Método Analítico}

\subsubsection{Caso Explícito}

Seja uma função $\varphi: \mathbf{R}^{\mathbf{m}} \mapsto \mathbf{R}^{\mathbf{p}}$ de classe $\mathcal{C}^{1}$, tal que para dois vetores $\mathbf{x} \in \mathbf{R}^{\mathbf{m}}$ e $\mathbf{y} \in \mathbf{R}^{\mathbf{p}}$ tem-se $\mathbf{y}=\varphi(\mathbf{x})$. A expansão de Taylor de primeira ordem de $\varphi \mathrm{em}$ torno de $\mathrm{E}[\mathbf{x}]$ é dada por:

$$
\varphi(\mathbf{x})=\varphi(\mathrm{E}[\mathbf{x}])+\mathrm{J}_{\varphi}(\mathrm{E}[\mathbf{x}])(\mathbf{x}-\mathrm{E}[\mathbf{x}])+\epsilon\left(\|\mathbf{x}-\mathrm{E}[\mathbf{x}]\|^{2}\right)
$$

onde a função $\epsilon: \mathbf{R} \mapsto \mathbf{R}^{\mathbf{p}}$ é tal que $\lim _{t \rightarrow \infty} \epsilon(t)=0$, e $\mathrm{J}_{\varphi}(\mathbf{x})=\partial \varphi(\mathbf{x}) / \partial \mathbf{x}$ é a matriz Jacobiana ${ }^{1}$ de $\varphi$. Considerando que cada amostra de $\mathbf{x}$ é suficientemente próxima de $\mathrm{E}[\mathbf{x}]$, pode-se aproximar $\varphi$ pelos termos de primeira ordem da equação (6.4), de forma que:

$$
\mathrm{E}[\mathbf{y}] \approx \varphi(\mathrm{E}[\mathbf{x}]) \quad \text { e } \quad \varphi(\mathbf{x})-\varphi(\mathrm{E}[\mathbf{x}]) \approx \mathrm{J}_{\varphi}(\mathrm{E}[\mathbf{x}])(\mathbf{x}-\mathrm{E}[\mathbf{x}])
$$

Assim, a aproximação de primeira ordem da matriz de covariância de y é dada em função da matriz de covariância de $\mathbf{x}$ :

$$
\Lambda_{\mathbf{y}} \approx \mathrm{E}\left[(\varphi(\mathbf{x})-\varphi(\mathrm{E}[\mathbf{x}]))(\varphi(\mathbf{x})-\varphi(\mathrm{E}[\mathbf{x}]))^{\mathrm{T}}\right] \approx \mathrm{J}_{\varphi}(\mathrm{E}[\mathbf{x}]) \Lambda_{\mathbf{x}} \mathrm{J}_{\varphi}(\mathrm{E}[\mathbf{x}])^{\mathrm{T}}
$$

\subsubsection{Caso Implícito}

Quando o vetor y é obtido através de minimização, a função $\varphi$ torna-se implícita, e a covariância de $\mathbf{y}$ é dada através da função que determina o critério de minimização. Conforme Faugeras (FAUGERAS, 1993), dada uma função de critério $\mathbf{C}: \mathbf{R}^{\mathbf{m}} \mapsto \mathbf{R}^{\mathbf{p}}$ de classe $\mathcal{C}^{\infty}$, seja $\mathbf{x}_{0} \in \mathbf{R}^{\mathbf{m}}$ o vetor de medidas e $\mathbf{y}_{0} \in \mathbf{R}^{\mathbf{p}}$ um mínimo local de $\mathrm{C}\left(\mathbf{x}_{0}, \mathbf{y}\right)$. Se a matriz Hessiana ${ }^{2}$ de $\mathbf{C}$ com respeito a $\mathbf{y}$ for inversivel em $(\mathbf{x}, \mathbf{y})=\left(\mathbf{x}_{0}, \mathbf{y}_{0}\right)$, então existe um conjunto aberto $\mathbf{U}^{\prime}$ de $\mathbf{R}^{\mathbf{m}}$ que contém $\mathbf{x}_{0}$, e um conjunto aberto $\mathbf{U}^{\prime \prime}$ de $\mathbf{R}^{\mathbf{p}}$ que contém $\mathbf{y}_{0}$, e uma relação $\varphi: \mathbf{R}^{\mathbf{m}} \mapsto \mathbf{R}^{\mathbf{p}}$ tal que para $(\mathbf{x}, \mathbf{y})$ em $\mathbf{U}^{\prime} \times \mathbf{U}^{\prime \prime}, \mathbf{y}_{0}=\varphi\left(\mathbf{x}_{0}\right)$. Ou seja, se $\mathbf{y}_{0}$ é um

\footnotetext{
${ }^{1}$ Matriz das derivadas parciais de primeira ordem de uma função vetorial.

${ }^{2}$ Matriz das derivadas parciais de segunda ordem de uma função vetorial.
} 
mínimo local da função de critério $\mathrm{C}(\mathbf{x}, \mathbf{y})$ avaliada em um vetor de medidas $\mathbf{x}_{0}$, então $\mathbf{y}_{0}=\varphi\left(\mathbf{x}_{0}\right)$. Adicionalmente, para $\Phi=\partial \mathrm{C} / \partial \mathbf{y}$ e $\mathrm{H}=\partial \Phi / \partial \mathbf{y}$, tem-se:

$$
\mathrm{J}_{\varphi}(\mathbf{x})=-\mathrm{H}^{-1} \frac{\partial \Phi}{\partial \mathbf{x}}
$$

Considerando $\mathbf{x}_{0}=\mathrm{E}[\mathbf{x}]$ e $\mathbf{y}_{0}=\mathrm{E}[\mathbf{y}]$, e aplicando (6.7) em (6.6), tem-se:

$$
\Lambda_{\mathbf{y}} \approx \mathrm{H}^{-1} \frac{\partial \Phi}{\partial \mathbf{x}} \Lambda_{\mathbf{x}} \frac{\partial \Phi^{\mathrm{T}}}{\partial \mathbf{x}} \mathrm{H}^{-\mathrm{T}}
$$

Função de Critério como Soma de Quadrados de Funções Implícitas. Considerando um conjunto de $n$ amostras $\hat{\mathbf{x}}_{i}$ do vetor aleatório $\hat{\mathbf{x}}$, e o vetor de medidas $\mathbf{x}=\left(\hat{\mathbf{x}}_{1}^{\mathrm{T}}, \ldots, \hat{\mathbf{x}}_{i}^{\mathrm{T}}, \ldots, \hat{\mathbf{x}}_{n}^{\mathrm{T}}\right)^{\mathrm{T}}$, seja uma função de critério $\mathrm{C}(\mathbf{x}, \mathbf{y})$ tal que:

$$
\mathrm{C}(\mathbf{x}, \mathbf{y})=\sum_{i=1}^{n} \mathrm{C}_{i}^{2}\left(\hat{\mathbf{x}}_{i}, \mathbf{y}\right)
$$

Desta forma, tem-se:

$$
\Phi=2 \sum_{i=1}^{n} \mathrm{C}_{i} \frac{\partial \mathrm{C}_{i}{ }^{\mathrm{T}}}{\partial \mathbf{y}} \quad \text { e } \quad \mathrm{H}=2 \sum_{i=1}^{n} \frac{\partial \mathrm{C}_{i}}{\partial \mathbf{y}} \frac{\partial \mathrm{C}_{i}{ }^{\mathrm{T}}}{\partial \mathbf{y}}+2 \sum_{i=1}^{n} \mathrm{C}_{i} \frac{\partial^{2} \mathrm{C}_{i}{ }^{\mathrm{T}}}{\partial \mathbf{y}^{2}}
$$

Normalmente os termos de segunda ordem são desprezíveis, e pode-se escrever:

$$
\mathrm{H} \approx 2 \sum_{i=1}^{n} \frac{\partial \mathrm{C}_{i}}{\partial \mathbf{y}} \frac{\partial \mathrm{C}_{i}{ }^{\mathrm{T}}}{\partial \mathbf{y}}
$$

Analogamente, tem-se:

$$
\frac{\partial \Phi}{\partial \mathbf{x}} \approx 2 \sum_{i=1}^{n} \frac{\partial \mathrm{C}_{i}{ }^{\mathrm{T}}}{\partial \mathbf{y}} \frac{\partial \mathrm{C}_{i}}{\partial \mathbf{x}}
$$

Desta forma, pode-se reescrever (6.8) como:

$$
\Lambda_{\mathbf{y}} \approx 4 \mathrm{H}^{-1} \sum_{i=1}^{n} \frac{\partial \mathrm{C}_{i}{ }^{\mathrm{T}}}{\partial \mathbf{y}} \frac{\partial \mathrm{C}_{i}}{\partial \mathbf{x}} \Lambda_{\mathbf{x}} \sum_{j=1}^{n} \frac{\partial \mathrm{C}_{j}{ }^{\mathrm{T}}}{\partial \mathbf{x}} \frac{\partial \mathrm{C}_{j}}{\partial \mathbf{y}}
$$

Assumindo que o ruído em $\hat{\mathbf{x}}_{i}$ é independente do ruído em $\hat{\mathbf{x}}_{i}$ para $i \neq j$, então $\Lambda_{\hat{\mathbf{x}}_{i} \hat{\mathbf{x}}_{j}}=\mathrm{E}\left[\left(\hat{\mathbf{x}}_{i}-\mathrm{E}\left[\hat{\mathbf{x}}_{j}\right]\right)\right]=0$ para $i \neq j$, e $\Lambda_{\mathbf{x}}=\operatorname{diag}\left(\Lambda_{\hat{\mathbf{x}}_{1}}, \Lambda_{\hat{\mathbf{x}}_{1}}, \ldots, \Lambda_{\hat{\mathbf{x}}_{n}}\right)$. Assim, a equação (6.13) pode ser escrita como: 


$$
\Lambda_{\mathbf{y}} \approx 4 \mathrm{H}^{-1} \sum_{i=1}^{n} \frac{\partial \mathrm{C}_{i}{ }^{\mathrm{T}}}{\partial \mathbf{y}} \frac{\partial \mathrm{C}_{i}}{\partial \mathbf{x}} \Lambda_{\mathbf{x}_{i}} \frac{\partial \mathrm{C}_{i}{ }^{\mathrm{T}}}{\partial \mathbf{x}} \frac{\partial \mathrm{C}_{i}}{\partial \mathbf{y}}
$$

Pela equação (6.6) tem-se $\Lambda_{\mathrm{C}_{i}}=\left(\partial \mathrm{C}_{i} / \partial \hat{\mathbf{x}}_{i}\right) \Lambda_{\mathbf{x}_{i}}\left(\partial \mathrm{C}_{i} / \partial \hat{\mathbf{x}}_{i}\right)^{\mathrm{T}}$, de onde segue que:

$$
\Lambda_{\mathbf{y}} \approx 4 \mathrm{H}^{-1} \sum_{i=1}^{n} \frac{\partial \mathrm{C}_{i}{ }^{\mathrm{T}}}{\partial \mathbf{y}} \Lambda_{\mathrm{C}_{i}} \frac{\partial \mathrm{C}_{i}}{\partial \mathbf{y}} \mathrm{H}^{-\mathrm{T}}
$$

Assumindo que a média das funções $\mathrm{C}_{i}$ é zero no ponto de mínimo, e que as funções $C_{i}$ são independentes entre si e de mesma distribuição de erro (condição em que a solução por mínimos-quadrados (SVD) é ótima), pode-se aproximar a covariância $\Lambda_{\mathrm{C}_{i}}$ pela variância das amostras (ANDERSON, 1958):

$$
\Lambda_{\mathrm{C}_{i}} \approx \frac{1}{n-p} \sum_{i=1}^{n} \mathrm{C}_{i}^{2} \approx \frac{\overline{\mathrm{C}}}{n-p},
$$

onde $\overline{\mathrm{C}}$ representa o valor da função de critério $\mathrm{C}$ no ponto de mínimo, e $p$ é o número de parâmetros (dimensão de y). Apesar do valor de $p$ ser desprezível para $n$ grande, quando $n=p$ sempre é possível encontrar um valor de $\mathbf{y}$ para o qual $\mathrm{C}_{i}=0$ para todo $i$. Neste caso não há significado em estimar-se a variância de $\mathrm{C}_{i}=0$, de forma que o fator $p$ corrige o efeito de conjuntos pequenos de amostras. Assim, pode-se reescrever a equação (6.15) como:

$$
\Lambda_{\mathbf{y}} \approx 2 \frac{\overline{\mathrm{C}}}{n-p} \mathrm{H}^{-1} \mathrm{HH}^{-\mathrm{T}}=2 \frac{\overline{\mathrm{C}}}{n-p} \mathrm{H}^{-\mathrm{T}} .
$$

Matriz Fundamental. A solução do sistema de equações de (5.2) corresponde a determinar a matriz fundamental $\mathrm{F}$ a partir da minimização de $\mathrm{C}(\tilde{\mathbf{x}}, \mathbf{f})=$ $\sum_{i=1}^{n}\left(\mathbf{x}_{i}^{\prime \mathrm{T}} \mathbf{F} \mathbf{x}_{i}\right)^{2}$, onde $\tilde{\mathbf{x}}=\left(\mathbf{x}_{1}{ }^{\mathrm{T}}, \mathbf{x}_{1}^{\prime \mathrm{T}}, \mathbf{x}_{2}{ }^{\mathrm{T}}, \mathbf{x}_{2}^{\prime \mathrm{T}}, \ldots, \mathbf{x}_{n}{ }^{\mathrm{T}}, \mathbf{x}_{n}^{\prime \mathrm{T}}\right)^{\mathrm{T}}$ e $\mathbf{f}$ é a forma vetorizada e parametrizada de F. Assim, a covariância $\Lambda_{\mathbf{f}}$ é dada pela equação (6.17), com a Hessiana obtida através do processo de minimização de $\mathrm{C}(\tilde{\mathbf{x}}, \mathbf{f})$. Segue pela equação (6.6) que a covariância da matriz fundamental $\Lambda_{F}$ é dada por:

$$
\Lambda_{\mathrm{F}}=\frac{\partial \mathrm{F}(\mathbf{f})}{\partial \mathbf{f}} \Lambda_{\mathbf{f}} \frac{\partial \mathrm{F}(\mathbf{f})^{\mathrm{T}}}{\partial \mathbf{f}}
$$

No caso do Algoritmo de 8 Pontos Correspondentes Normalizado, o vetor f tem dimensão 9, uma vez que a restrição de singularidade é imposta após a minimização. A solução de $\mathrm{F}$ é dada a menos de um fator de escala, e o vetor $\mathbf{f}$ é normalizado de forma que $\|\mathbf{f}\|=1$, e $\mathbf{F}(\mathbf{f})$ reflete esta normalização. No caso do Algoritmo de 7 Pontos Correspondentes, a solução de F é exata, e não há 
necessidade de se determinar a covariância. No entanto, quando se considera a busca exaustiva pela melhor solução deste algoritmo, a função critério consiste do erro residual dado pela equação (5.4), e pode-se determinar $\Lambda_{\mathrm{F}}$ a partir das equações (6.17) e (6.18). 


\section{Reconstrução Métrica}

A reconstrução consiste em determinar as matrizes de câmera $\mathrm{P}$ e $\mathrm{P}^{\prime}$, bem como os pontos $3 \mathrm{D} \mathbf{X}_{i}$ tais que:

$$
\mathbf{x}_{i}=\mathrm{PX}_{i} \quad \mathrm{e} \quad \mathbf{x}_{i}^{\prime}=\mathrm{P}^{\prime} \mathbf{X}_{i}
$$

para um conjunto de pontos correspondentes entre duas imagens $\left\{\mathbf{x}_{i} \leftrightarrow \mathbf{x}_{i}^{\prime}\right\}$, onde tanto os pontos $3 \mathrm{D}\left\{\mathbf{X}_{i}\right\}$ como a posição, orientação e calibração das câmeras $\left(\mathrm{P}, \mathrm{P}^{\prime}\right)$ são desconhecidos.

Dado um número suficiente de pares de pontos correspondentes pode-se utilizar um dos algoritmos descritos no Capítulo 5 para calcular a matriz fundamental, e a cena pode ser determinada a menos de uma transformação projetiva. Esta reconstrução projetiva baseia-se no par de matrizes de câmera canônicas, apresentado na Seção 4.4.3. A ambigüidade reconstrutiva inerente deste método, descrita na Seção 4.4.4, pode ser reduzida mediante informações adicionais sobre as câmeras ou sobre as cenas. Em particular, dado um número suficiente de pontos 3D cujas coordenadas Euclidianas sejam conhecidas, o teorema da reconstrução projetiva, apresentado na Seção 4.4.5, determina que é possível obter a reconstrução métrica da cena.

O método de reconstrução métrica de uma cena a partir de duas imagens consiste basicamente na determinação da reconstrução projetiva canônica a partir da matriz fundamental, seguida de uma retificação para reconstrução métrica a partir de pontos do espaço tridimensional cujas coordenadas Euclidianas sejam conhecidas. Assim, o algoritmo de reconstrução métrica envolve os seguintes elementos: (1) determinação da matriz fundamental, (2) determinação das matrizes de projeção canônicas, (3) triangulação de pontos correspondentes das imagens a partir destas matrizes, e (4) determinação das matrizes de projeção métricas a partir das coordenadas Euclidianas daqueles pontos.

Os conceitos apresentados neste capítulo foram extraídos de (HARTLEY; ZIS- 
SERMAN, 2000), (FAUGERAS, 1993), (UllMAN, 1979), e (XU; ZHANG, 1996). A notação adotada e as ilustrações das figuras são de (HARTLEY; ZISSERMAN, 2000).

\subsection{Matriz Fundamental}

Conforme visto anteriormente, dado um conjunto de $N \geq 7$ pontos correspondentes $\mathbf{x}_{i} \leftrightarrow \mathbf{x}_{i}^{\prime}$ entre duas imagens, determina-se a matriz fundamental $\mathrm{F}$ a partir da condição de correspondência $\mathbf{x}_{i}^{\prime \mathrm{T}} \mathrm{F} \mathbf{x}_{i}$. Os métodos de determinação da matriz fundamental estão descritos detalhadamente no Capítulo 5, e deve-se escolher aquele que melhor se adapte ao conjunto de pontos correspondentes disponível, pela quantidade e pelas características de ruído das medidas, e que tenha o melhor desempenho segundo as limitações computacionais do sistema em que será utilizado.

\subsection{Câmeras Canônicas}

Um par de câmeras canônicas $\left(\mathrm{P}_{\mathrm{C}}, \mathrm{P}_{\mathrm{C}}^{\prime}\right)$ pode ser derivado diretamente da matriz fundamental F pela equação (4.16), p.52. Conforme demonstrado na Seção 4.4.2, existe uma ambigüidade projetiva quanto à determinação destas câmeras, de forma que, por simplicidade, escolhe-se o par de câmeras canônicas dado por:

$$
\mathrm{P}_{\mathrm{C}}=[\mathrm{I} \mid \mathbf{0}] \quad \mathrm{P}_{\mathrm{C}}^{\prime}=\left[\left[\mathbf{e}^{\prime}\right]_{\times} \mathrm{F} \mid \mathbf{e}^{\prime}\right]
$$

\subsection{Triangulação}

Dados o par de câmeras $\left(\mathrm{P}, \mathrm{P}^{\prime}\right)$ e um par de pontos correspondentes $\mathbf{x} \leftrightarrow \mathbf{x}^{\prime}$ que satisfazem a condição de correspondência $\mathrm{x}^{\prime \mathrm{T}} \mathrm{F} \mathbf{x}=0$, foi demonstado no Capítulo 4 que os dois raios da projeção inversa de $\mathbf{x}$ e $\mathbf{x}^{\prime}$ encontram-se em um ponto $3 \mathrm{D} \mathbf{X}$, que por sua vez é projetado nos pontos $\mathbf{x}$ e $\mathbf{x}^{\prime}$ originais pelas câmeras P e P', respectivamente. A Figura 7.1 ilustra esta construção, denominada triangulação.

Os únicos pontos do espaço tridimensional que não podem ser determinados pelo método de triangulação a partir de suas imagens são aqueles sobre a reta base entre as duas câmeras. Neste caso os raios da projeção inversa são colineares, e interceptam-se em todos os pontos de suas extensões.

$\mathrm{O}$ ponto $\mathbf{X}$ pode ser determinado a partir de suas imagens $\mathbf{x}$ e $\mathbf{x}^{\prime}$ e do par 


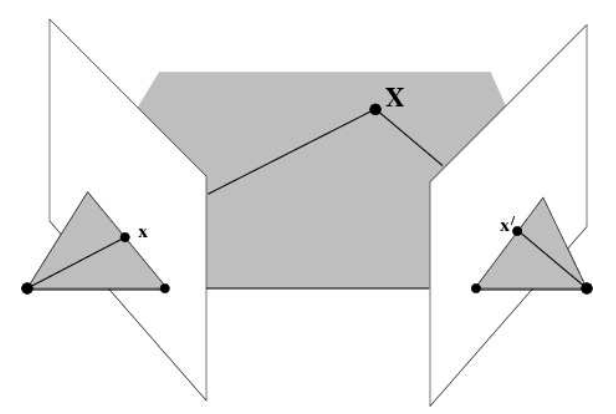

Figura 7.1: Triangulação.

de câmeras $\left(\mathrm{P}, \mathrm{P}^{\prime}\right)$ por triangulação linear, através de um método derivado do algoritmo DLT (Apêndice A, Seção A.8). Para cada imagem, tem-se $\mathbf{x}=$ PX e $\mathbf{x}^{\prime}=\mathrm{P}^{\prime} \mathbf{X}$, e estas equações podem ser combinadas para formar um sistema linear homogêneo $\mathrm{AX}=\mathbf{0}$.

Inicialmente, elimina-se o fator de escala homogêneo através do produto vetorial, resultando em três equações para cada ponto de imagem, duas das quais são linearmente independentes. No caso da primeira imagem, $\mathbf{x} \times \mathbf{P X}=\mathbf{0}$, e para $\mathbf{x}=(x, y, 1)^{\mathrm{T}}$ tem-se:

$$
\begin{gathered}
x\left(\mathbf{p}^{3^{\mathrm{T}}} \mathbf{X}\right)-\left(\mathbf{p}^{1^{\mathrm{T}}} \mathbf{X}\right)=0 \\
y\left(\mathbf{p}^{3^{\mathrm{T}}} \mathbf{X}\right)-\left(\mathbf{p}^{2^{\mathrm{T}}} \mathbf{X}\right)=0 \\
x\left(\mathbf{p}^{2^{\mathrm{T}}} \mathbf{X}\right)-y\left(\mathbf{p}^{1^{\mathrm{T}}} \mathbf{X}\right)=0
\end{gathered}
$$

onde os vetores $\mathbf{p}^{i^{\mathrm{T}}}$ representam as linhas de P. Derivando-se equações análogas para o ponto $\mathbf{x}^{\prime}=\left(x^{\prime}, y^{\prime}, 1\right)^{\mathrm{T}}$ da segunda imagem, tem-se:

$$
\mathrm{AX}=\left[\begin{array}{c}
x \mathbf{p}^{3^{\mathrm{T}}}-\mathbf{p}^{1^{\mathrm{T}}} \\
y \mathbf{p}^{3^{\mathrm{T}}}-\mathbf{p}^{2^{\mathrm{T}}} \\
x^{\prime} \mathbf{p}^{3^{\mathrm{T}}}-\mathbf{p}^{\prime 1^{\mathrm{T}}} \\
y^{\prime} \mathbf{p}^{3^{\mathrm{T}}}-\mathbf{p}^{\prime 2^{\mathrm{T}}}
\end{array}\right] \mathbf{X}=\mathbf{0}
$$

onde a matriz A é composta de duas equações de cada imagem, resultando em quatro equações sobre os quatro elementos do vetor homogêneo $\mathbf{X}$. Trata-se de um sistema de equações sobre-determinado, uma vez que a solução é dada a menos de um fator de escala. Desta forma, o sistema de equações em (7.4) pode ser resolvido através da SVD de A, e a solução $\mathbf{X}$ corresponde ao vetor singular relativo ao menor valor singular de $\mathrm{A}$. 


\subsection{Câmeras Métricas}

Pode-se passar da reconstrução projetiva, determinada pelas matrizes de projeção canônicas $\left(\mathrm{P}_{\mathrm{C}}, \mathrm{P}_{\mathrm{C}}^{\prime}\right)$, diretamente para a reconstrução métrica, $\left(\mathrm{P}_{\mathrm{E}}, \mathrm{P}_{\mathrm{E}}^{\prime}\right)$, dado um conjunto com um número suficiente de pontos de referência - pontos $3 \mathrm{D}$ cujas coordenadas Euclidianas são conhecidas.

Considerando um conjunto de $n$ pontos de referência 3D $\left\{\mathbf{X}_{\mathrm{E} i}\right\}$, as projeções destes pontos em um par de imagens são representadas pelo conjunto de pontos correspondentes $\left\{\mathbf{x}_{i} \leftrightarrow \mathbf{x}_{i}^{\prime}\right\}$. A partir destas correspondências, determina-se o conjunto de pontos $3 \mathrm{D}\left\{\mathbf{X}_{\mathrm{C} i}\right\}$, reconstruídos por triangulação a partir das câmeras canônicas $\left(\mathrm{P}_{\mathrm{C}}, \mathrm{P}_{\mathrm{C}}^{\prime}\right)$. Pelo teorema da reconstrução projetiva, Seção 4.4.5, $p .55$, estes pontos da reconstrução projetiva estão relacionados com os pontos de referência por uma homografia:

$$
\mathbf{X}_{\mathrm{E} i}=\mathrm{HX}_{\mathrm{C} i} \quad i=1, \ldots, n
$$

Cada correspondência $\mathbf{x}_{i} \leftrightarrow \mathbf{x}_{i}^{\prime}$ fornece três equações independentes em função dos elementos de $\mathrm{H}$. Como visto anteriormente, $\mathrm{H}$ tem 15 graus de liberdade, de forma que obtém-se uma solução linear para $n \geq 5$ - contanto que não haja nenhuma combinação de quatro destes pontos pertencendo a um único plano.

Denominando $\mathbf{h}^{j^{\mathrm{T}}}$ a $j$-ésima linha de $\mathrm{H}$, em coordenadas homogêneas, por (4.20), tem-se:

$$
\mathbf{X}_{\mathrm{E} i}=\mathrm{H}_{\mathbf{C} i}=\left(\begin{array}{c}
\mathbf{h}^{{ }^{\mathrm{T}}} \mathbf{X}_{\mathrm{C} i} \\
\mathbf{h}^{2^{\mathrm{T}}} \mathbf{X}_{\mathrm{C} i} \\
\mathbf{h}^{3 \mathrm{~T}} \mathbf{X}_{\mathrm{C} i} \\
\mathbf{h}^{4^{\mathrm{T}}} \mathbf{X}_{\mathrm{C} i}
\end{array}\right)
$$

Em coordenadas não-homogêneas, o pode-se escrever:

$$
\tilde{\mathbf{X}}_{\mathrm{E} i}=\left(\begin{array}{c}
x_{{ }_{\mathrm{E} i}} \\
x_{2}{ }_{\mathrm{E} i} \\
x_{3{ }_{\mathrm{E} i}}
\end{array}\right)=\frac{1}{\mathbf{h}^{4^{\mathrm{T}}} \mathbf{X}_{\mathrm{C} i}}\left(\begin{array}{c}
\mathbf{h}^{1^{\mathrm{T}}} \mathbf{X}_{\mathrm{C} i} \\
\mathbf{h}^{2^{\mathrm{T}}} \mathbf{X}_{\mathrm{C} i} \\
\mathbf{h}^{3^{\mathrm{T}}} \mathbf{X}_{\mathrm{C} i}
\end{array}\right)
$$

onde o vetor $\tilde{\mathbf{X}}_{\mathrm{E} i}=\left(x_{1_{\mathrm{E} i}}, x_{\mathrm{E}_{\mathrm{E} i}}, x_{3_{\mathrm{E} i}}\right)^{\mathrm{T}}$ é a representação do ponto $\mathbf{X}_{\mathrm{E} i}$ em coordenadas não-homogêneas. Desta forma, para cada coordenada Euclidiana do ponto $\tilde{\mathbf{X}}_{\mathrm{E} i}$, tem-se: 


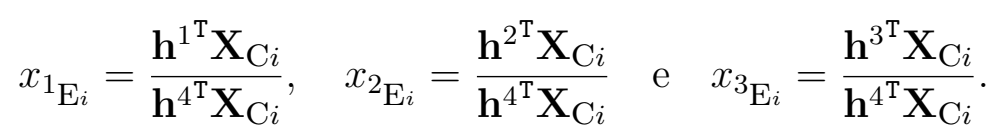

Assim, para cada ponto de referência $3 \mathrm{D} \mathbf{X}_{\mathrm{E} i}$, tem-se um sistema de três equações independentes dado por:

$$
\mathrm{A}_{i} \mathbf{h}=\left[\begin{array}{cccc}
-\mathbf{X}_{\mathrm{C} i}^{\mathrm{T}} & \mathbf{0}^{\mathrm{T}} & \mathbf{0}^{\mathrm{T}} & x_{1}{ }_{\mathrm{E} i} \mathbf{X}_{\mathrm{C} i}^{\mathrm{T}} \\
\mathbf{0}^{\mathrm{T}} & -\mathbf{X}_{\mathrm{C} i}^{\mathrm{T}} & \mathbf{0}^{\mathrm{T}} & x_{2} \mathbf{X}_{\mathrm{E} i}^{\mathrm{T}} \\
\mathbf{0}^{\mathrm{T}} & \mathbf{0}^{\mathrm{T}} & -\mathbf{X}_{\mathrm{C} i}^{\mathrm{T}} & x_{3 \mathrm{E} i} \mathbf{X}_{\mathrm{C} i}^{\mathrm{T}}
\end{array}\right]\left(\begin{array}{c}
\mathbf{h}^{1} \\
\mathbf{h}^{2} \\
\mathbf{h}^{3} \\
\mathbf{h}^{4}
\end{array}\right)=\mathbf{0}
$$

onde $\mathbf{h}=\left(\mathbf{h}^{1^{\mathrm{T}}}, \mathbf{h}^{2^{\mathrm{T}}}, \mathbf{h}^{3^{\mathrm{T}}}, \mathbf{h}^{4^{\mathrm{T}}}\right)^{\mathrm{T}}$ é o vetor dos elementos da matriz $\mathrm{H}$, e $\mathrm{A}_{i}$ é uma matriz $3 \times 16$.

Para o conjunto de $n$ pontos de referência $3 \mathrm{D} \mathbf{X}_{\mathrm{E} i}$ tem-se um sistema de $3 n$ equações por 16 incógnitas, de forma que é possível encontrar uma solução para $n \geq 5$. A solução de $\mathbf{h}$ é dada através da SVD de $\mathbf{A}=\left[\mathrm{A}_{i}\right]$, a matriz $3 n \times 16$ definida pelos $n$ pontos de referência $3 \mathrm{D} \mathbf{X}_{\mathrm{E} i}$, e corresponde ao vetor singular relativo ao menor valor singular de $\mathrm{A}$.

\subsection{Erro de Reconstrução}

Analogamente ao erro residual apresentado na Seção 5.5, pode-se avaliar o algoritmo de reconstrução através do erro de reconstrução: soma das distâncias euclidianas quadráticas entre os ponto de referência e os pontos reconstruídos. Esta medida de erro pode ser escrita como:

$$
\text { erec }=\sum_{i} \mathrm{~d}\left(\mathbf{X}_{\mathrm{E} i}, \mathbf{X}_{\mathrm{R} i}\right)^{2}=\sum_{i}\left(\mathbf{X}_{\mathrm{E} i}-\mathbf{X}_{i}\right)^{\mathrm{T}}\left(\mathbf{X}_{\mathrm{E} i}-\mathbf{X}_{\mathrm{R} i}\right)
$$

onde o vetor $\mathbf{X}_{\mathrm{R} i}$ representa o ponto 3D reconstruído por triangulação a partir das matrizes de projeção métricas e dos pontos correspondentes relativos ao ponto de referência $\mathbf{X}_{\mathrm{E} i}$.

\subsection{Algoritmo de Reconstrução}

Dadas duas imagens não calibradas, o Algoritmo de Reconstrução determina a reconstrução métrica $\left(\mathrm{P}_{\mathrm{M}}, \mathrm{P}_{\mathrm{M}}^{\prime},\left\{\mathbf{X}_{\mathrm{M}}\right\}\right)$ das câmeras e da estrutura, através dos seguintes passos: 
1. Cálculo da Reconstrução Projetiva $\left(\mathrm{P}_{\mathrm{C}}, \mathrm{P}_{\mathrm{C}}^{\prime},\left\{\mathrm{X}_{\mathrm{C}}\right\}\right)$.

(a) Cálculo da Matriz Fundamental. Determina-se a matriz fundamental $\mathrm{F}$ a partir de pares de pontos correspondentes $\mathbf{x} \leftrightarrow \mathbf{x}^{\prime}$, utilizando-se um dos algoritmos descritos no Capítulo 5.

(b) Cálculo das Matrizes de Câmera. Determinam-se as matrizes de câmera canônicas $\mathrm{P}_{\mathrm{C}}$ e $\mathrm{P}_{\mathrm{C}}^{\prime}$ a partir da matriz fundamental, segundo a equação (7.2).

(c) Triangulação. Para cada par de pontos correspondentes $\mathbf{x} \leftrightarrow \mathbf{x}^{\prime}$ calcula-se o ponto $3 \mathrm{D} \mathbf{X}_{\mathrm{C} i}$ por triangulação a partir das câmeras $\mathrm{P}_{\mathrm{C}} \mathrm{e}$ $\mathrm{P}_{\mathrm{C}}^{\prime}$

2. Retificação da reconstrução projetiva para métrica. Determina-se a homografia $\mathrm{H}$ tal que $\mathbf{X}_{\mathrm{E} i}=\mathrm{HX}_{\mathrm{C} i}$ para um conjunto de pontos de referência $3 \mathrm{D}\left\{\mathbf{X}_{\mathrm{E} i}\right\}$, cujas coordenadas Euclidianas são conhecidas. A reconstrução métrica é dada por:

$$
\mathrm{P}_{\mathrm{M}}=\mathrm{P}_{\mathrm{C}} \mathrm{H}^{-1}, \quad \mathrm{P}_{\mathrm{M}}^{\prime}=\mathrm{P}_{\mathrm{C}^{\prime}}^{\prime} \mathrm{H}^{-1}, \quad \mathbf{X}_{\mathrm{M} i}=\mathrm{HX}_{\mathrm{C} i} .
$$

Este algoritmo sumariza os conceitos apresentados nas seções anteriores, fornecendo um método completo para estimação de posição a partir da geometria epipolar entre imagens. 


\section{Complexidade Computacional}

A complexidade computacional de um algoritmo fornece a dimensão do custo de processamento, em termos de diferentes recursos computacionais, conforme descrito no Apêndice A, Seção A.9. A complexidade computacional temporal é de especial interesse neste trabalho, pois representa um fator crítico para escolha de algoritmos quando se considera sua utilização em aplicações de tempo real, como o caso de um robô autônomo. Os resultados apresentados neste capítulo baseiam-se em conceitos apresentados em (LEWIS; PAPADIMITRIOU, 1997).

Considerando os algoritmos descritos no Capítulo 5, todos utilizam SVD de uma matriz $n \times 9$, $n$ variando de 7 ao tamanho total do conjunto de pares de pontos correspondentes entre duas imagens. Cada um dos algoritmos apresentados terá passos adicionais de complexidade variável, porém sempre polinomial. No caso do método de determinação das matrizes de câmeras canônicas e métricas, a complexidade computacional é respectivamente constante e dependente da SVD de uma matriz $n \times 15, n \geq 15$. De maneira geral, um algoritmo para o cálculo da SVD de uma matriz formada por $n$ vetores-linha de $k$ elementos pertence a $\mathcal{O}\left(k^{2} n\right)$. Por questão de simplicidade, consideram-se apenas as operações matemáticas - todas com o mesmo custo - nos cálculos da complexidade computacional apresentados a seguir. A Tabela 8.1 sumariza os resulados deste capítulo.

Tabela 8.1: Complexidade computacional de algoritmos de cálculo da matriz fundamental e de reconstrução métrica.

\begin{tabular}{|c|c|c|}
\hline \hline \multirow{2}{*}{ Algoritmo } & \multicolumn{2}{|c|}{ Complexidade Computacional } \\
\cline { 2 - 3 } & Matriz Fundamental & Reconstrução Métrica \\
\hline 8 Pontos Correspondentes Normalizado & $\mathcal{O}(n)$ & $\mathcal{O}(n+k)$ \\
\hline 7 Pontos Correspondentes & $\mathcal{O}(1)$ & $\mathcal{O}(k)$ \\
\hline Busca Exaustiva & $\mathcal{O}\left(n^{8}\right)$ & $\mathcal{O}\left(n^{8}+k\right)$ \\
\hline Solução Linear Iterativa & $\mathcal{O}(K n)$ & $\mathcal{O}(K n+k)$ \\
\hline \hline$n$ é o número de correspondências, $k$ é o número de pontos de referência, \\
e $K$ é o número máximo de iterações. \\
\hline
\end{tabular}




\subsection{Algoritmo de 8 Pontos Correspondentes Nor- malizado}

O Algoritmo de 8 Pontos Correspondentes Normalizado, descrito na Seção 5.4, consiste de quatro passos principais: (1) normalização dos dados de entrada nas duas imagens, (2) SVD do sistema de $n$ equações definido em (5.2), (3) determinação da matriz fundamental que satisfaz à restrição de singularidade através de SVD, e (4) cálculo da matriz fundamental para o conjunto de pontos original. O primeiro passo, normalização, envolve o cálculo do centróide dos $n$ pontos média das coordenadas dos pontos - e corresponde a $2 n$ operações. Para determinar os fatores de escala que garantem que a distância média dos pontos à origem seja $\sqrt{2}$, é necessário transladar o centróide para a origem ( $2 n$ operações) e determinar a média das normas de cada ponto ( $4 n$ operações), totalizando $24 n$ operações, considerando ambas imagens. A construção das matrizes de transformação envolve multiplicação de matrizes $3 \times 3$, totalizando 90 operações em ambas imagens. Para normalizar o conjunto de pontos a partir das matrizes de transformação são necessárias $30 n$ operações. Portanto, o total desta etapa é $54 n+90$ operações. A SVD tem complexidade $(O)\left(k^{2} n\right)$, e neste caso $k=9$. A imposição da restrição de singularidade requer a $\operatorname{SVD}$ de $\mathrm{F}=\operatorname{Udiag}(r, s, t) \mathbf{V}^{\mathrm{T}}$, e posterior reconstrução $\mathrm{F}^{\prime}=\operatorname{Udiag}(r, s, 0) \mathbf{V}^{\mathrm{T}}$, tem complexidade constante. Finalmente, o cálculo da matriz fundamental correspondente ao conjunto de pontos originais corresponde a multiplicação de duas matrizes $3 \times 3$, que requer 90 operações. Desta forma, a complexidade computacional do Algoritmo de 8 Pontos Correspondentes Normalizado é $\mathcal{O}(n)$.

\subsection{Algoritmo de 7 Pontos Correspondentes}

O Algoritmo de 7 Pontos Correspondentes, descrito na Seção 5.3, utiliza SVD de uma matriz $7 \times 9$, que apresenta complexidade computacional constante. A determinação do fator $\alpha$ tal que det $\left(\alpha \mathrm{F}_{1}+(1-\alpha) \mathrm{F}_{2}\right)=0$ representa a solução de uma equação polinomial em $\alpha^{3}$. O cálculo das raízes de um polinômio de terceiro grau pode ser feito numericamente ou analiticamente, caso em que serão necessárias 86 operações para determinação da raiz real e 90 operações para determinação das raízes complexas. O cálculo dos coeficientes do polinômio característico de $\operatorname{det}\left(\alpha \mathrm{F}_{1}+(1-\alpha) \mathrm{F}_{2}\right)$ envolve 121 operações, e o cálculo de cada uma das três soluções possíveis para a matriz fundamental requer 28 operações. Desta forma, a complexidade computacional do Algoritmo de 7 Pontos Correspondentes é con- 
stante, $\mathcal{O}(1)$.

\subsection{Erro Residual}

O cálculo do erro residual segundo a equação (5.4) requer determinar $\mathbf{l}_{i}=\mathrm{F}^{\mathrm{T}} \mathbf{x}_{i}^{\prime}$ e $\mathbf{l}_{i}^{\prime}=\mathbf{F x}_{i}$ para cada par de pontos correspondentes $\mathbf{x}_{i} \leftrightarrow \mathbf{x}_{i}^{\prime}$, representando 15 operações cada. Adicionalmente, o cálculo das distâncias d $\left(\mathbf{x}_{i}, \mathbf{l}_{i}\right)$ e d $\left(\mathbf{x}_{i}^{\prime}, \mathbf{l}_{i}^{\prime}\right)$ requer 9 operações cada. Então para cada par de pontos correspondentes tem-se um total de 51 operações para determinação da distância quadrática composta. Assim, o cálculo do erro residual tem complexidade computacional $\mathcal{O}(n)$.

\subsection{Busca Exaustiva}

A complexidade computacional de cada iteração do Algoritmo de Busca Exaustiva, descrito na Seção 5.6, corresponde à complexidade computacional do Algoritmo de 7 Pontos Correspondentes, acrescida da complexidade computacional do cálculo do erro residual para as três possíveis soluções de F fornecidas por esse método. O número de iterações total do algoritmo para um conjunto de $n$ pares de pontos correspondentes é $K=n ! /(7 !(n-7) !)$ - o número combinações de $n$ pontos 7 a 7 . Este valor é uma função polinomial $7^{\circ}$ na variável $n$, $K=\left(n^{7}-21 n^{6}+175 n^{5}-735 n^{4}+1624 n^{3}-1764 n^{2}+720 n\right) / 5040$. Desta forma, a complexidade computacional do Algoritmo de Busca Exaustiva é $\mathcal{O}\left(n^{8}\right)$.

\subsection{Solução Linear Iterativa}

No caso do Algoritmo Linear Iterativo, descrito na Seção 5.7, a complexidade computacional de cada iteração corresponde à complexidade do Algoritmo de 8 Pontos Correspondentes Normalizado acrescida da complexidade do cálculo dos coeficientes $k_{i}$ para cada um dos $n$ pares de pontos correspondentes, e do produto deste coeficiente pela linha correspondente da matriz A definida na equação (5.2). O cálculo do coeficiente $k_{i}$ corresponde à raiz quadrada da soma dos quadrados das distâncias epipolares dos pontos $\mathbf{x}_{i}$ e $\mathbf{x}_{i}^{\prime}$, que requer $52 n$ operações. A ponderação das $n$ equações de A requer $9 n$ operações. Assim, cada iteração requer $196 n+297$ operações. Assim, denominando $K$ o número máximo de iterações do algoritmo, a complexidade computacional da Solução Linear Iterativa é $\mathcal{O}(K n)$. 


\subsection{Reconstrução}

O Algoritmo de Reconstrução, descrito na Seção 7.6, consiste basicamente de quatro etapas: cálculo da matriz fundamental, cálculo das matrizes de projeção canônicas, triangulação dos pontos correspondentes relativos aos pontos de referência 3D, e cálculo da homografia de retificação para reconstrução métrica. A complexidade computacional da etapa de cálculo da matriz fundamental depende da escolha do algoritmo, podendo assumir um dos valores descritos nas seções anteriores deste capítulo. O cálculo das matrizes de projeção canônicas de (7.2) requer a multiplicação de duas matrizes $3 \times 3$ (45 operações), e a determinação do epipolo da segunda imagem, que corresponde à solução de um sistema homogêneo de três equações em três incógnitas. A solução de um sistema de $n$ equações e $n$ incógnitas por eliminação de Gauss, tem complexidade $\mathcal{O}\left(n^{3}\right)$, e pode ser considerado um valor representativo para esta operação. Desta forma, como esta etapa consiste de um número constante de opreações, o cálculo das matrizes de projeção canônicas tem complexidade constante. A etapa de triangulação é composta pela determinação do sistema de equações em (7.4), que requer 24 operações, e pela solução deste sistema por SVD, com complexidade $\mathcal{O}(1)$. Se o conjunto de pontos de referência 3D tem $k$ pontos, a complexidade total da etapa de triangulação é $\mathcal{O}(k)$. A etapa de cálculo retificação da reconstrução projetiva para métrica requer a construção de $k$ sistemas de equações como em (7.9), com $12 k$ operações. Assim, a determinação da homografia $\mathrm{H}$ depende da SVD de uma matriz $3 k \times 16$, de complexidade $\mathcal{O}(k)$. A determinação das matrizes de projeção métricas requer duas multiplicações de matrizes $4 \times 3$ e $4 \times 4$, totalizando 168 operações. Desta forma, a complexidade computacional da etapa de retificação da reconstrução projetiva para métrica é $\mathcal{O}(k)$. Assim, considerando $n$ pares de pontos correspondentes para determinação da matriz fundamental e $k$ pontos de referência 3D, a complexidade computacional da Retificação Métrica é $\mathcal{O}(n+k)$ para matriz fundamental determinada pelo Algoritmo de 8 Pontos Correspondentes Normalizado; $\mathcal{O}(k)$ para matriz fundamental determinada pelo Algoritmo de 7 Pontos Correspondentes; $\mathcal{O}\left(n^{8}+k\right)$ para matriz fundamental determinada pelo Algoritmo de Busca exaustiva; e $\mathcal{O}(K n+k)$ para matriz fundamental determinada pelo Algoritmo Linear Iterativo. 


\section{Parte II}

\section{Resultados Práticos}




\section{Resultados}

Como visto anteriormente, os métodos de estimação de posição por geometria epipolar baseiam-se na determinação da matriz fundamental da correspondência entre duas imagens de uma cena - através de um dos algoritmos apresentados no Capítulo 5. A partir da matriz fundamental, pode-se determinar diretamente as matrizes de projeção canônicas - uma primeira estimativa de posição, que pode indicar algumas características do movimento da câmera. Com o acréscimo de pontos de referência de coordenadas 3D conhecidas, a partir das matrizes de projeção canônicas pode-se determinar as matrizes de projeção métricas e obter a estimativa de posição da câmera em relação ao sistema de coordenadas global. Os métodos consistem portanto de três etapas: (1) determinação matriz fundamental, (2) cálculo da reconstrução projetiva e (3) cálculo da reconstrução métrica - cada qual com sua parcela de incertezas influenciando o erro global da estimativa de posição. A aplicabilidade de cada método e a comparação entre eles foi realizada através da análise do erro observado quando aplicados a conjuntos de dados experimentais, levando-se em conta o cálculo da complexidade computacional. Com isso tem-se um panorama de qual o melhor método de estimação, quais as situações em que este método pode ser utilizado, e o grau de confiabilidade das estimativas obtidas através do mesmo.

\subsection{Conjuntos de Dados}

Os métodos de estimação de posição por geometria epipolar utilizam conjuntos de pares de pontos correspondentes entre duas imagens para determinação da matriz fundamental e das matrizes de projeção canônicas, e conjuntos de pontos de referência do espaço 3D e suas projeções nestas imagens para determinação das matrizes de projeção métricas.

Os conjuntos de pares de pontos correspondentes utilizados para determinação da matriz fundamental foram obtidos de duas formas distintas: através de simulação - imagens geradas por computador e pontos correspondentes definidos 


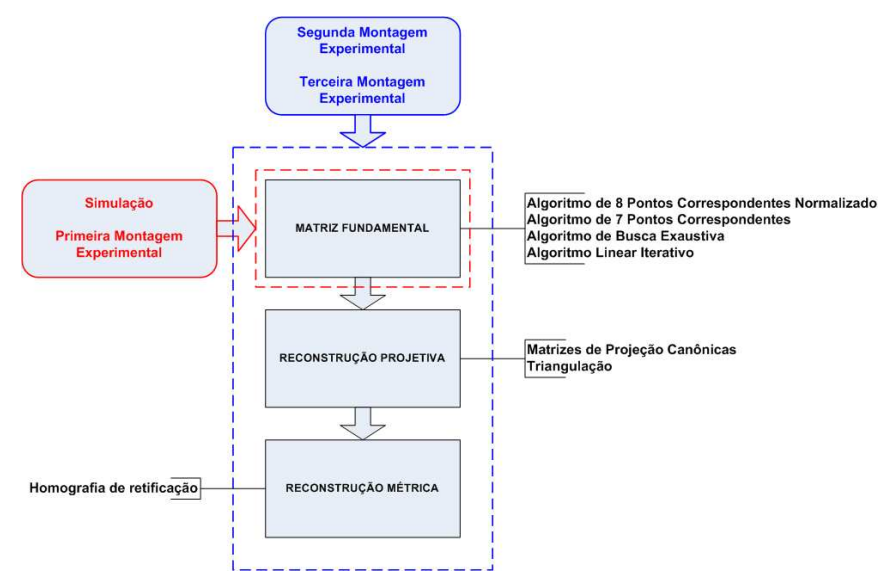

Figura 9.1: Conjuntos de dados utilizados na estimação de posição.

computacionalmente, e através de montagens experimentais - imagens tomadas através de câmeras digitais e pontos correspondentes definidos empiricamente. Em ambos os casos, as imagens utilizadas para obtenção de tais conjuntos de pontos correspondentes representam seqüências de uma mesma cena tomadas de uma câmera em movimento. Os movimento de câmera considerados englobam rotações e translações quaisquer - movimento geral - e casos particulares de translação pura e movimento planar, descritos na Seção 4.3.

Os pontos de referência utilizados na determinação das matrizes de projeção métricas foram obtidos através de medidas de posição de objetos nas cenas das imagens de montagens experimentais, sendo que para os conjuntos de dados de simulação as matrizes de projeção são conhecidas a priori.

A Figura 9.1 mostra o processo de estimação de posição por geometria epipolar e a utilização dos conjuntos de dados delineados nas próximas seções neste processo.

\subsubsection{Simulação}

Os conjuntos de dados de simulação foram gerados através do software MATLAB ${ }^{\circledR}$ 6.5 , sendo que os pontos correspondentes entre duas imagens representando um determinado movimento de câmera foram calculados diretamente das matrizes de projeção determinadas a partir da rotação e translação de cada câmera em relação à origem do sistema de coordenadas global, conforme a equação (3.10). Dentre os parâmetros intrínsecos do modelo de câmera pontual, foi considerada apenas a distância focal, escolhida de forma a enquadrar corretamente a cena em cada imagem. 


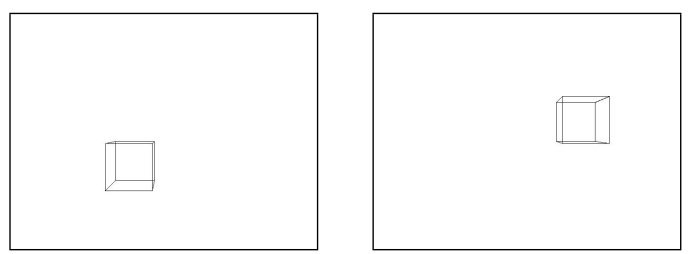

(a)

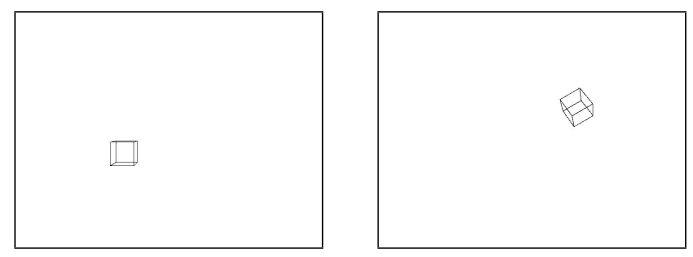

(b)

Figura 9.2: Imagens do conjunto de dados de simulação. (a) Movimento de translação pura. (b) Movimento planar.

As imagens dos conjuntos de dados de simulação representam dois movimentos de câmera particulares: translação pura e movimento planar, estudados na Seção 4.3. No caso da translação pura, a câmera se desloca nas três coordenadas entre a primeira e a segunda imagem, mas não há rotação em relação a qualquer dos eixos do sistema de coordenadas global. No caso do movimento planar, existe apenas rotação em torno do eixo Z e translação nas coordenadas X e Y globais. Em ambos os casos a cena consiste de um cubo de 200 pixel de lado centrado na origem do sistema de coordenadas global, e os conjuntos de dados são formados por 20 pares de pontos correspondentes escolhidos aleatoriamente sobre as projeções das arestas deste cubo em cada imagem, de 640x480 pixel de resolução cada, exibidas na Figura 9.2.

Estes dados foram utilizados para comparar o desempenho quanto ao erro e à complexidade dos algoritmos de determinação da matriz fundamental, pois fornecem medidas livres de ruído, onde pode-se observar o erro devido à quantização da imagem e à precisão numérica do cálculo. Além destes erros, neste conjunto de dados observa-se também o efeito do condicionamento do sistema de equações (5.2) em relação à escolha de correspondências entre as imagens.

\subsubsection{Montagem Experimental}

Estes conjuntos de dados foram tomados de três montagens experimentais distintas, onde as imagens foram obtidas a partir de uma câmera digital realizando um movimento conhecido. Os conjuntos de pontos correspondentes de cada par de imagens considerado foram determinados empiricamente com auxílio de um programa desenvolvido no software MATLAB ${ }^{\circledR} 6.5$. 


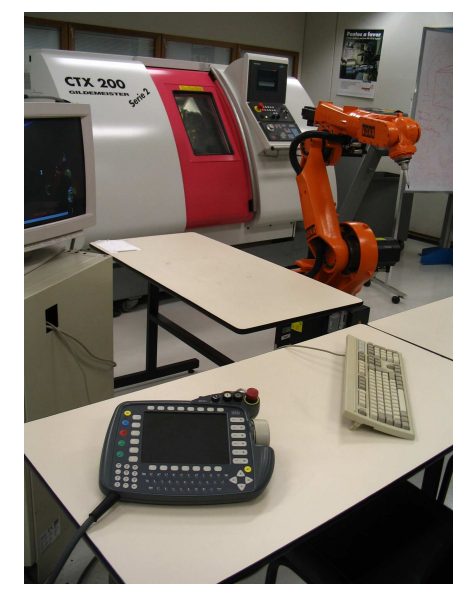

Figura 9.3: O robô KUKA no ambiente do laboratório.

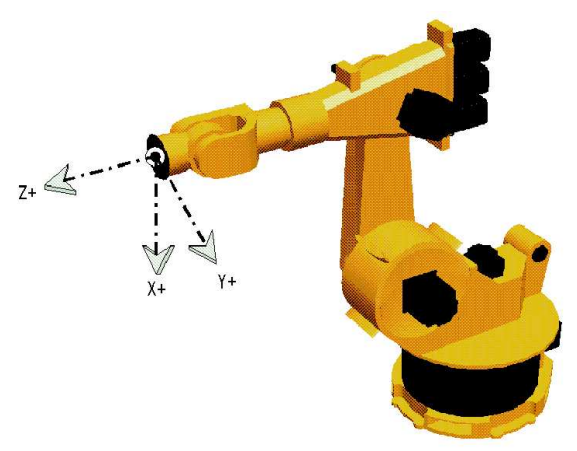

(a)

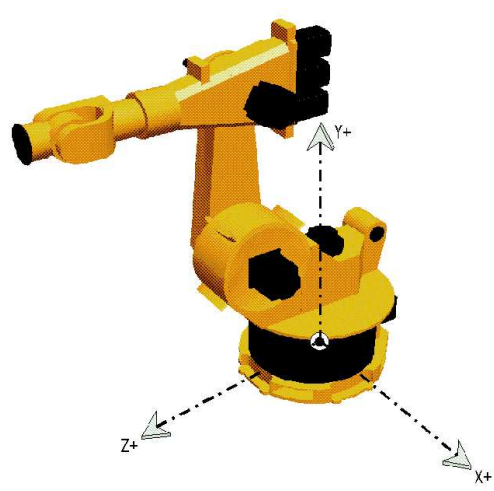

(b)

Figura 9.4: Sistemas de coordenadas do robô KUKA, conforme o manual. (a) Sistema de coordenadas na flange. (b) Sistema de coordenadas na base.

De forma a obter um movimento controlado e conhecido da câmera, esta foi acoplada à extremidade da flange do braço robô KUKA do Departamento de Eng. ${ }^{a}$ de Controle e Automação Mecânica. Este robô permite realizar movimentos complexos com controle total da posição da flange, seja manualmente ou através de um programa de trajetória. Assim, a posição da câmera em cada imagem coletada nas montagens experimentais fica perfeitamente definida em termos da translação e rotação em relação a um sistema de coordenadas global localizado na base do robô. A Figura 9.3 mostra o robô KUKA no ambiente do laboratório.

Foram utilizadas duas câmeras diferentes para obtenção das imagens das montagens experimentais: uma câmera fotográfica digital comum de lente fixa e uma câmera digital FireWire com lentes intercambiáveis de diferentes comprimentos focais. O controle das câmeras e a captura das imagens em computador foi realizada através de software específico que acompanha cada câmera.

Na primeira montagem experimental foi utilizada uma câmera fotográfica di- 


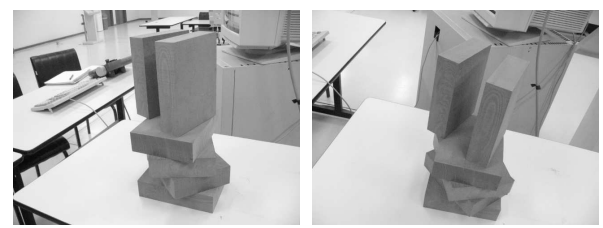

(a)

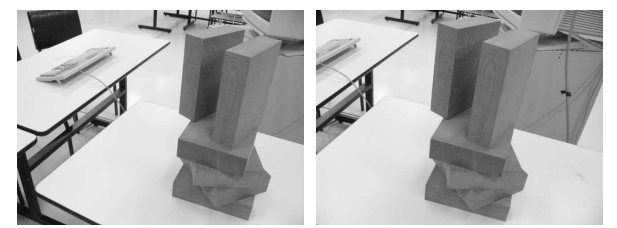

(b)

Figura 9.5: Imagens da primeira montagem experimental. (a) Movimento de translação pura. (b) Movimento geral.

gital Canon modelo PowerShot A75, configurada com foco fixo e resolução de 640x480 pixel. Foi utilizado o software de controle e aquisição Canon Utilities RemoteCapture, que permite acionar a câmera e capturar imagens estáticas diretamente no computador através da interface USB. A Figura 9.5 mostra a seqüência de imagens obtida através desta câmera, tomada posicionando-se a flange do robô segundo o movimento desejado e acionando-se a câmera manualmente através do software de controle. Neste caso o controle do robô foi realizado utilizando o sistema de coordenadas na flange, conforme mostrado na Figura 9.4(a).

Os conjuntos de dados referentes a cada par de imagens consideradas nesta montagem experimental são formados por 20 pares de pontos correspondentes, escolhidos visualmente através do programa de auxílio gráfico em MATLAB ${ }^{\circledR}$ 6.5 .

Os dados desta primeira montagem experimental foram utilizados em conjunto com os dados de simulação para comparar o desempenho quanto ao erro e à complexidade dos algoritmos de determinação da matriz fundamental. A análise dos resultados desta montagem experimental em paralelo com a análise dos resultados de simulação permite observar o efeito da incerteza da matriz fundamental e do condicionamento do sistema de equações (5.2) em relação à escolha de correspondências entre as imagens.

Nas demais montagens experimentais foi utilizada uma câmera FireWire monocromática ImagingSource modelo DMK31BF03, com CCD progressive scan de 1/3", 1024x768 pixel, freqüência de amostragem de até 30 quadros/s, e interface para trigger externo. A lente utilizada foi um modelo Pentax C815B(KA) de distância focal $8.5 \mathrm{~mm}$, que permite uma visão abrangente do laboratório e com foco em distâncias médias e distorção aceitável das bordas. O software de controle e aquisição desta câmera é o ICCapture 2.0 da ImagingSource, que permite controlar todas as funcionalidades da câmera, acionar a captura de imagens estáticas ou de seqüências com variadas taxas de amostragem e ainda capturar imagens em 

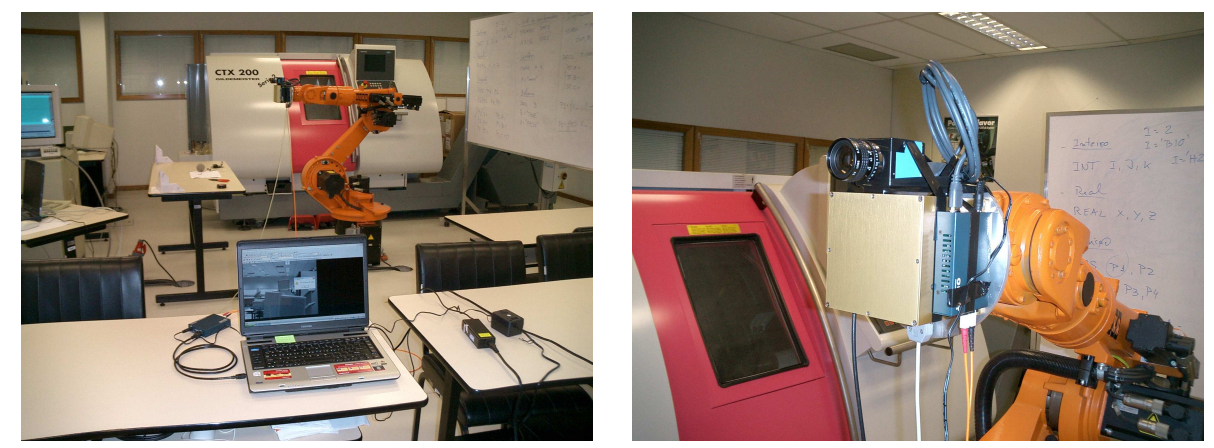

Figura 9.6: Segunda montagem experimental.

sincronia com um trigger externo. As montagens experimentais com a câmera FireWire utilizaram cabos de fibra-óptica e alimentação externa da câmera para permitir um maior alcance do movimento do robô. Também foi utilizado o acionamento por meio de um sinal de trigger, gerado automaticamente pela unidade de controle do robô, segundo os programas de trajetória de cada uma destas montagens experimentais. As trajetórias programadas no robô KUKA foram definidas segundo o sistema de coordenadas em sua base, conforme mostrado na Figura 9.4(b).

Os pontos de referência utilizados para o cálculo das matrizes de projeção métricas foram determinados medindo-se as coordenadas de diversos elementos presentes nas cenas da segunda e da terceira montagem experimental, como as coordenadas dos cantos da sala do laboratório e de estruturas como portas, janelas, luminárias, saídas de ar, painéis, alçapões e objetos com contorno ou marcações bem definidas. Estas medidas foram tomadas utilizando-se uma trena comum, de forma que sua acuidade refletisse as condições dos ambientes em que se pretende usar um sistema de visão computacional. As projeções destes pontos nas imagens dos conjuntos de dados destas duas montagens foram determinados através do mesmo programa em MATLAB ${ }^{\circledR} 6.5$ utilizado para determinar os pontos correspondentes para o cálculo da matriz fundamental.

Na segunda montagem experimental foi programada uma trajetória no robô de forma a realizar movimento de translação pura nas coordenadas X e Y, segundo o sistema de coordenadas na base do robô (Figura 9.4(b)). O acionamento da câmera foi feito automaticamente através do software de controle, a cada ponto de interesse da trajetória, pelo sinal de trigger proveniente da central de controle do robô KUKA. A Figura 9.6 mostra esta montagem experimental, e a Figura 9.7 mostra a seqüência de imagens obtida.

Os dados desta segunda montagem experimental foram utilizados para ana- 
lisar o comportamento dos algoritmos de determinação da matriz fundamental e de reconstrução métrica em uma situação em que o movimento é limitado a um plano paralelo ao plano de imagem da câmera.

Na terceira montagem experimental foi programada uma trajetória no robô de forma a realizar movimentos variados, incluindo seqüências de translação pura, movimento planar - horizontal, vertical e sobre um plano qualquer, e movimento geral. O acionamento da câmera foi feito automaticamente através do software de controle, a cada ponto de interesse da trajetória, pelo sinal de trigger proveniente da central de controle do robô KUKA. A Figura 9.8 mostra esta montagem experimental, e a Figura 9.9 mostra a seqüência de imagens obtida.

Os conjuntos de dados referentes a cada par de imagens consideradas na segunda e terceira montagens experimentais são formados por 32 pares de pontos correspondentes e 5 ou mais pontos de referência 3D e suas projeções nas imagens - tomando-se o cuidado de não haverem quatro deles coplanares. Para análise do erro na estimativa de posição foram escolhidos alguns pontos de referência adicionais e suas projeções em cada par de imagens, alguns próximos aos pontos de referência utilizados na determinação das matrizes de projeção métricas e outros em outras regiões da cena. Como na primeira montagem, estes pontos foram escolhidos visualmente através do programa de auxílio gráfico em MATLAB ${ }^{\circledR}$ 6.5 .

Os dados desta terceira e última montagem experimental foram utilizados para analisar o algoritmo de reconstrução métrica como um todo, emulando uma seqüência genérica de movimentos. Estes resultados permitem verificar o desempenho do algoritmo em relação ao erro e complexidade, bem como determinar as condições para sua aplicação.
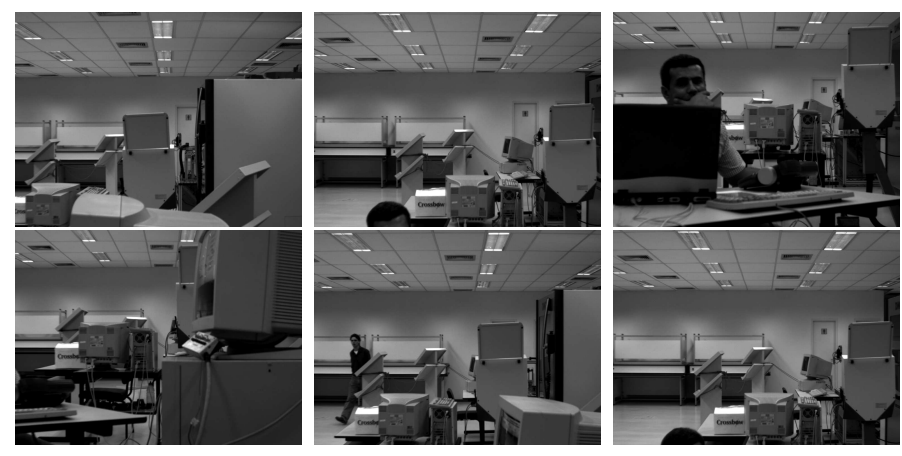

Figura 9.7: Imagens da segunda montagem experimental. 


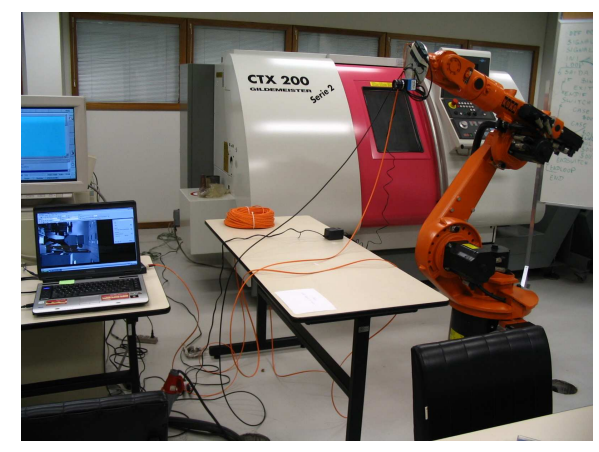

Figura 9.8: Terceira montagem experimental.
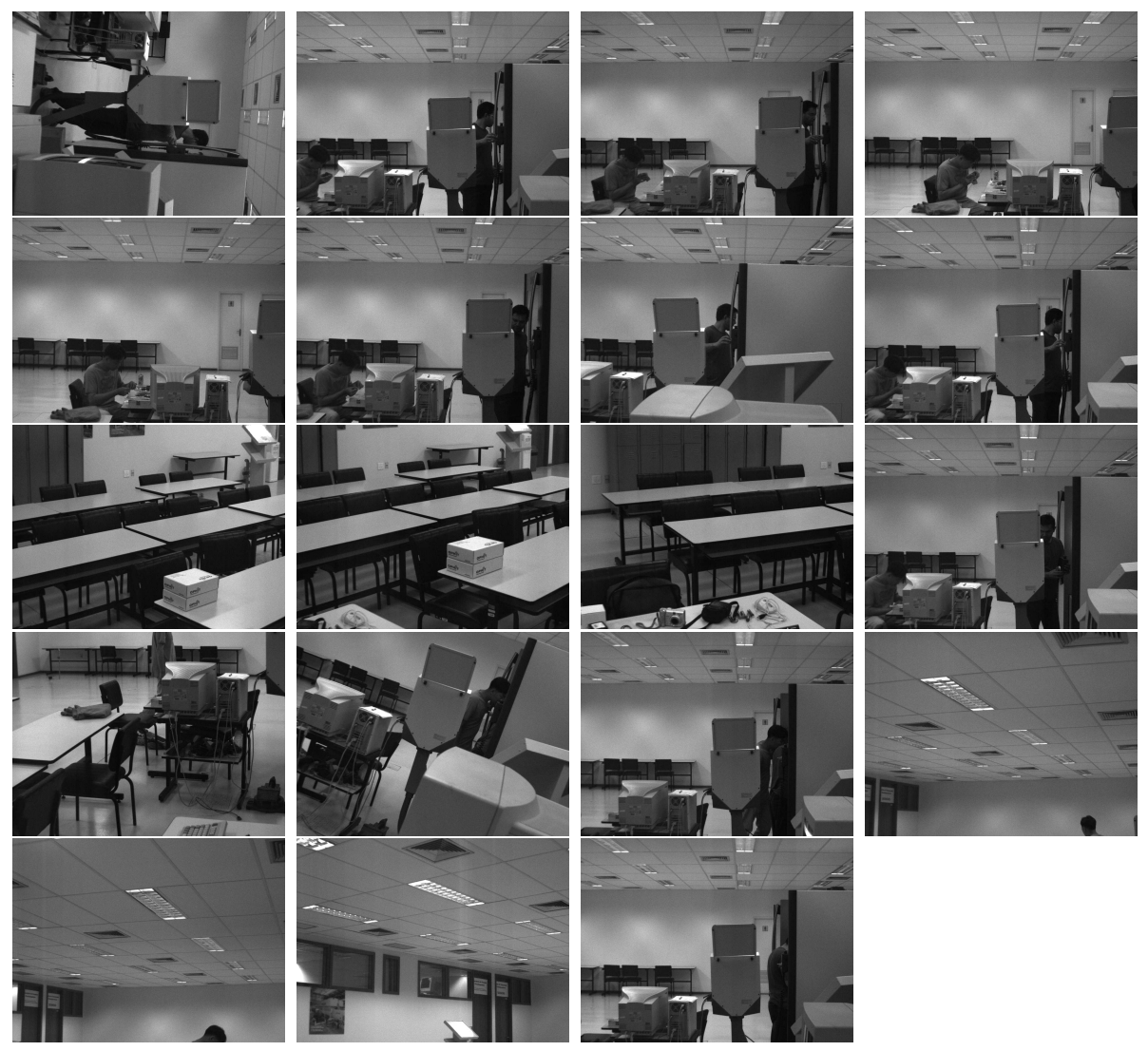

Figura 9.9: Imagens da terceira montagem experimental. 


\subsection{Processamento}

O processamento dos conjuntos de dados experimentais foi realizado através de programas desenvolvidos em software MATLAB ${ }^{\circledR}$ 6.5. Cada etapa da estimação de posição - determinação da matriz fundamental, determinação das matrizes de projeção canônicas e determinação das matrizes de projeção métricas - foi realizada por uma série de rotinas e funções desenvolvidas de forma a determinar os resultados e os erros envolvidos em cada processo. Assim, em cada etapa, os dados coletados foram divididos em duas partes, uma utilizada para verificar a validade dos resultados obtidos através da outra. Um dos programas desenvolvidos consiste de uma interface gráfica para auxílio na determinação de pontos correspondentes, utilizada tanto para obtenção dos pares de pontos utilizada na determinação da matriz fundamental como para obtenção das projeções dos pontos de referência em cada imagem dos conjuntos de dados da segunda e terceira montagens experimentais. Um outro programa foi desenvolvido para exibir de forma gráfica os resultados dos cálculos da matriz fundamental e dos pontos correspondentes ou de referência, na forma de pontos correspondentes e respectivas retas epipolares. Os demais programas são todos não interativos, desenvolvidos para o processamento em lote dos vários conjuntos de dados. As listagens dos programas encontram-se no Apêndice B.

\subsubsection{Matriz Fundamental}

O cálculo da matriz fundamental foi realizado segundo os algoritmos apresentados no Capítulo 5: algoritmo de 7 Pontos Correspondentes, algoritmo de 8 Pontos Correspondentes Normalizado, algoritmo de Busca Exaustiva e algoritmo Linear Iterativo. No caso dos conjuntos de dados de simulação e da primeira montagem experimental, foram selecionados aleatoriamente subconjuntos de 7, 8, 10 e 15 pares de pontos correspondentes para o cálculo da matriz fundamental, dentre os 20 pares de pontos correspondentes totais marcados em cada par de imagens. No caso das segunda e terceira montagens experimentais, os 32 pares de pontos correspondentes totais marcados em cada par de imagens foram subdivididos aleatoriamente em subconjuntos complementares: de 16 pares de pontos correspondentes cada - denominados A e B, e de 7 e 25 pares de pontos correspondentes - denominados C e D. Os subconjuntos C e D foram utilizados respectivamente para o cálculo da matriz fundamental pelo Algoritmo de 7 Pontos Correspondentes e seu erro residual, enquanto os subconjuntos A e B foram utilizados alternadamente como entrada e para o cálculo do erro residual dos demais algoritmos de 
Tabela 9.1: Erro residual da simulação $\left(\right.$ pixel $\left.^{2}\right)$.

\begin{tabular}{|l|r|r|r|r|}
\hline Algoritmo & \multicolumn{2}{|c|}{ Translação Pura } & \multicolumn{2}{c|}{ Movimento Planar } \\
\cline { 2 - 5 } & $\begin{array}{r}\text { Conjunto de } \\
\text { Entrada }\end{array}$ & $\begin{array}{r}\text { Conjunto } \\
\text { Completo }\end{array}$ & $\begin{array}{r}\text { Conjunto de } \\
\text { Entrada }\end{array}$ & $\begin{array}{r}\text { Conjunto } \\
\text { Completo }\end{array}$ \\
\hline \hline 7 Pontos - 1 a solução & 0.0000 & 0.4401 & 0.0000 & 0.0809 \\
\hline 7 Pontos - 2 ${ }^{\text {a }}$ solução & 0.0000 & 32.9524 & 0.0000 & 0.9377 \\
\hline 7 Pontos - 3 solução & 0.0000 & 515.4311 & 0.0000 & 3.8259 \\
\hline Busca Exaustiva - 8 entradas & 0.0018 & 0.0946 & 0.0000 & 0.0231 \\
\hline Busca Exaustiva - 10 entradas & 0.0000 & 0.1502 & 0.0001 & 0.0015 \\
\hline Busca Exaustiva - 15 entradas & 0.0061 & 0.0450 & 0.0006 & 0.0032 \\
\hline 8 Pontos Normalizado - 8 entradas & 0.6362 & 14.5389 & 0.0636 & 5.8422 \\
\hline 8 Pontos Normalizado - 10 entradas & 0.0000 & 0.1545 & 0.0002 & 0.0012 \\
\hline 8 Pontos Normalizado - 15 entradas & 0.0177 & 0.1984 & 0.0006 & 0.0010 \\
\hline Linear Iterativo - 8 entradas & 0.6362 & 14.5389 & 0.0636 & 5.8422 \\
\hline Linear Iterativo - 10 entradas & 0.0000 & 0.1545 & 0.0002 & 0.0012 \\
\hline Linear Iterativo - 15 entradas & 0.0177 & 0.1984 & 0.0006 & 0.0010 \\
\hline
\end{tabular}

determinação da matriz fundamental.

O conjunto de dados de simulação consiste de quatro imagens, duas formando o par relativo ao movimento de translação pura e duas formando o par relativo ao movimento planar. O conjunto de dados da primeira montagem experimental consiste também de quatro imagens, nomeadas 01 a 03, formando um par representando movimento de translação pura (01-02), e duas formando um par representando movimento geral (03-04).

A Tabela 9.1 mostra o erro residual obtido da aplicação de cada algoritmo de determinação da matriz fundamental ao conjunto de dados de simulação. Os valores são referentes ao erro residual do subconjunto de pares de pontos correspondentes de entrada e do conjunto completo de pares de pontos correspondentes, para cada par de imagens $\left(1^{\circ}\right.$ par de imagens - movimento de translação pura; segundo par de imagens - movimento planar em XY). A Tabela 9.2 apresenta a mesma comparação de erro residual para o conjunto de dados da primeira montagem experimental ( $1^{\circ}$ par de imagens - movimento de translação pura; segundo par de imagens - movimento geral). Em ambos os casos o erro residual é dado em frações de pixel ao quadrado.

As Figuras 9.10 e 9.11 mostram os resultados dos algoritmos de determinação da matriz fundamental aplicados ao conjunto de dados de teste, respectivamente para os casos de movimento de translação pura e movimento planar em XY. As figuras representam a superposição de cada par de imagens do conjunto de dados, com os pontos correspondentes e as retas epipolares obtidas a partir de cada algoritmo de determinação da matriz fundamental marcados sobre as mesmas. Analogamente, as Figuras 9.12 e 9.13 mostram os resultados dos algoritmos de determinação da matriz fundamental aplicados ao conjunto de dados da primeira montagem experimental, respectivamente para os casos de movimento de 
Tabela 9.2: Erro residual da primeira montagem experimental $\left(\operatorname{pixel}^{2}\right)$.

\begin{tabular}{|c|c|c|c|c|}
\hline \multirow[t]{2}{*}{ Algoritmo } & \multicolumn{2}{|c|}{ Translação Pura } & \multicolumn{2}{|c|}{ Movimento Geral } \\
\hline & $\begin{array}{r}\text { Conjunto de } \\
\text { Entrada }\end{array}$ & $\begin{array}{l}\text { Conjunto } \\
\text { Completo }\end{array}$ & $\begin{array}{r}\text { Conjunto de } \\
\text { Entrada }\end{array}$ & $\begin{array}{l}\text { Conjunto } \\
\text { Completo }\end{array}$ \\
\hline 7 Pontos $-1^{\mathrm{a}}$ solução & 0.0000 & 0.0144 & $0.0000 \mathrm{E}-4$ & 0.0004 \\
\hline 7 Pontos $-2^{a}$ solução & 0.0000 & 0.4278 & $0.0000 \mathrm{E}-4$ & 0.0011 \\
\hline 7 Pontos $-3^{a}$ solução & 0.0000 & 1.8355 & $0.0000 \mathrm{E}-4$ & 0.0035 \\
\hline Busca Exaustiva - 8 entradas & 0.0000 & 0.0005 & $0.0135 \mathrm{E}-4$ & 0.0002 \\
\hline Busca Exaustiva - 10 entradas & 0.0001 & 0.0005 & $0.1500 \mathrm{E}-4$ & 0.0001 \\
\hline Busca Exaustiva - 15 entradas & 0.0001 & 0.0002 & $0.2220 \mathrm{E}-4$ & 0.0000 \\
\hline 8 Pontos Normalizado - 8 entradas & 0.0006 & 0.0038 & $0.5906 \mathrm{E}-4$ & 0.0002 \\
\hline 8 Pontos Normalizado - 10 entradas & 0.0021 & 0.0060 & $0.3784 \mathrm{E}-4$ & 0.0001 \\
\hline 8 Pontos Normalizado - 15 entradas & 0.0006 & 0.0020 & $0.6115 \mathrm{E}-4$ & 0.0001 \\
\hline Linear Iterativo - 8 entradas & 0.0006 & 0.0038 & $0.5906 \mathrm{E}-4$ & 0.0002 \\
\hline Linear Normalizado Iterativo - 10 entradas & 0.0019 & 0.0056 & $0.4025 \mathrm{E}-4$ & 0.0001 \\
\hline Linear Normalizado Iterativo - 15 entradas & 0.0004 & 0.0011 & $0.9948 \mathrm{E}-4$ & 0.0001 \\
\hline
\end{tabular}

translação pura e movimento geral. Em ambos os casos, os blocos de resultados do Algoritmo de 7 Pontos Correspondentes apresentam as três soluções possíveis, enquanto os blocos dos demais algoritmos apresentam soluções obtidas a partir de conjuntos de entrada com 8, 10 e 15 pares de pontos correspondentes.

O conjunto de dados da segunda montagem experimental é composto por uma seqüência de seis imagens nomeadas 00 a 05, tomadas em pares seqüenciais e alternados. Apesar de ser possível encontrar pares de pontos correspondentes suficientes para o uso de qualquer dos algoritmos de determinação da matriz fundamental para cada um destes pares de imagens, alguns foram descartados por não ter sido possível encontrar pontos de referência com projeções em ambas imagens suficientes para o cálculo das matrizes de projeção métricas. Dessa forma, o conjunto de dados efetivamente utilizado nos cálculos é composto dos pares de imagens 01-02, 02-03, 04-05, 01-04, 02-04 e 02-05. Alguns destes pares de imagens são bastante semelhantes mas as posições não são exatamente iguais.

A Tabela 9.3 mostra o erro residual obtido com o Algoritmo de 7 Pontos Correspondentes para cada par de imagens do conjunto de dados da segunda montagem experimental. O erro residual foi calculado em relação aos pares de pontos correspondentes do subconjunto de entrada e com relação aos pares de pontos correspondentes do subconjunto complementar ao mesmo. Analogamente, as Tabelas 9.4, 9.5 e 9.6 apresentam o erro residual obtido respectivamente com o Algoritmo de Busca Exaustiva, o Algoritmo de 8 Pontos Correspondentes Normalizado e o Algoritmo Linear Iterativo, aplicados ao conjunto de dados da segunda montagem experimental. Em todos os casos, o valor do erro residual é dado em frações de pixel ao quadrado.

As Figuras 9.14 a 9.17 mostram os resultados dos algoritmos de cálculo da matriz fundamental aplicados ao conjunto de dados da segunda montagem ex- 

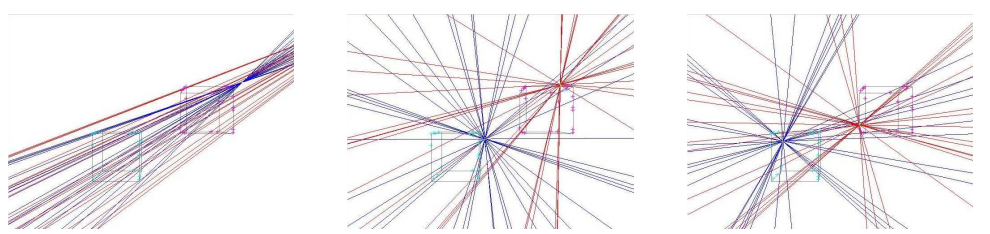

(a)
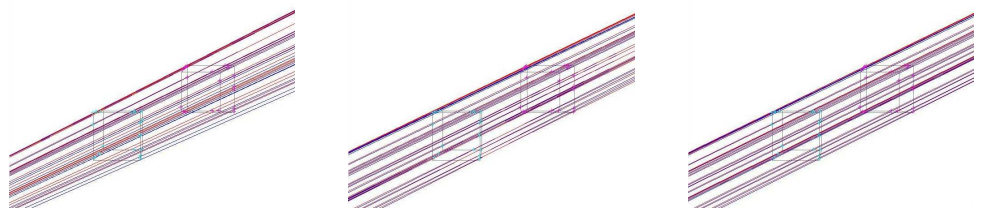

(b)
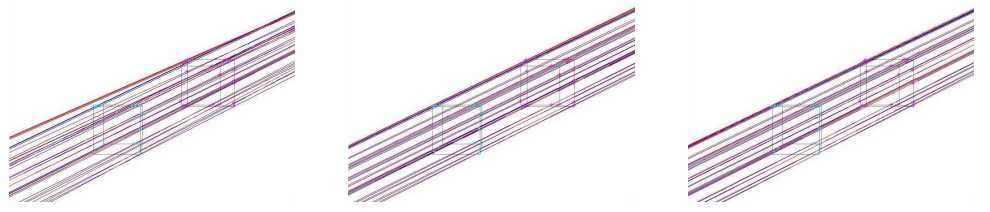

(c)
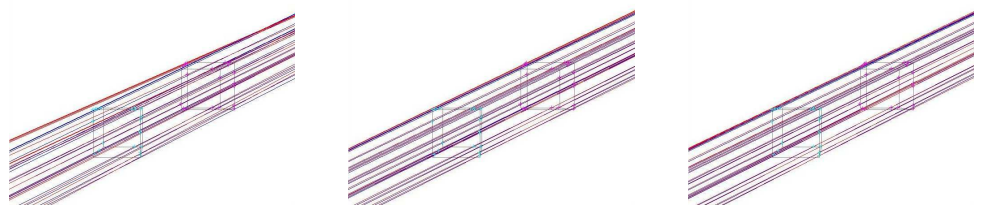

(d)

Figura 9.10: Resultados de simulação para movimento de translação pura. (a) Algoritmo de 7 Pontos Correspondentes. (b) Algoritmo de Busca Exaustiva. (c) Algoritmo de 8 Pontos Correspondentes Normalizado. (d) Algoritmo Linear Iterativo.

Tabela 9.3: Erro residual do Algoritmos de 7 Pontos Correspondentes na segunda montagem experimental $\left(\mathrm{pixel}^{2}\right)$.

\begin{tabular}{|l|r|r|r|r|r|r|}
\hline \multirow{2}{*}{ Imagens } & \multicolumn{2}{|c|}{$1^{\mathrm{a}}$ Solução } & \multicolumn{2}{c|}{$2^{\mathrm{a}}$ Solução } & \multicolumn{2}{c|}{$3^{\mathrm{a}}$ Solução } \\
\cline { 2 - 7 } & $\begin{array}{r}\text { Conjunto de } \\
\text { Entrada }\end{array}$ & $\begin{array}{r}\text { Conjunto } \\
\text { Complementar }\end{array}$ & $\begin{array}{r}\text { Conjunto de } \\
\text { Entrada }\end{array}$ & $\begin{array}{r}\text { Conjunto } \\
\text { Complementar }\end{array}$ & $\begin{array}{r}\text { Conjunto de } \\
\text { Entrada }\end{array}$ & $\begin{array}{r}\text { Conjunto } \\
\text { Complementar }\end{array}$ \\
\hline \hline $01-02$ & $9.5898 \mathrm{E}-27$ & 12.1342 & $5.1324 \mathrm{E}-26$ & 40.1761 & $1.1013 \mathrm{E}-26$ & 11.1075 \\
\hline $02-03$ & $9.2285 \mathrm{E}-26$ & 20.2977 & $9.8861 \mathrm{E}-27$ & 27.3348 & $1.8152 \mathrm{E}-26$ & 51.0161 \\
\hline $04-05$ & $1.6146 \mathrm{E}-25$ & 3.6599 & $5.1025 \mathrm{E}-26$ & 43.3775 & $6.7265 \mathrm{E}-26$ & 12.5171 \\
\hline $01-04$ & $9.2285 \mathrm{E}-26$ & 20.2977 & $9.8861 \mathrm{E}-27$ & 27.3348 & $1.8152 \mathrm{E}-26$ & 51.0161 \\
\hline $01-05$ & \multicolumn{6}{|c|}{ soluções inexistentes } \\
\hline $02-04$ & $9.6551 \mathrm{E}-28$ & 8.4077 & $5.7812 \mathrm{E}-28$ & 8.5038 & $3.43203 \mathrm{E}-27$ & 14.2690 \\
\hline $02-05$ & $6.9535 \mathrm{E}-28$ & 73.1024 & $3.8950 \mathrm{E}-28$ & 7.4036 & $3.4095 \mathrm{E}-28$ & 10.4331 \\
\hline
\end{tabular}



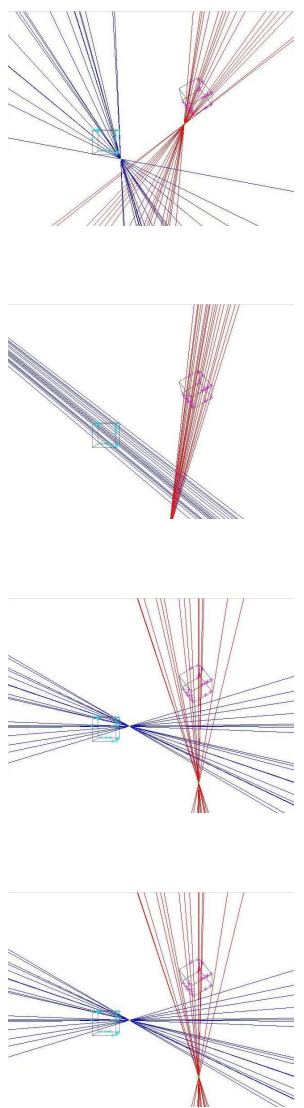

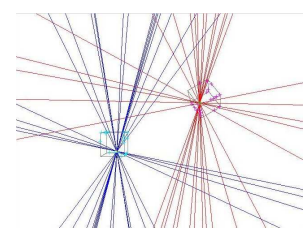

(a)

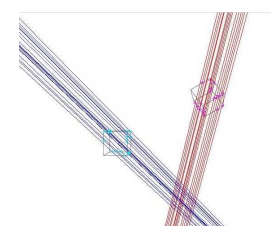

(b)

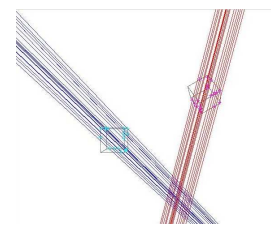

(c)

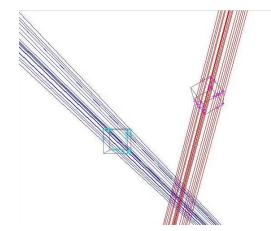

(d)
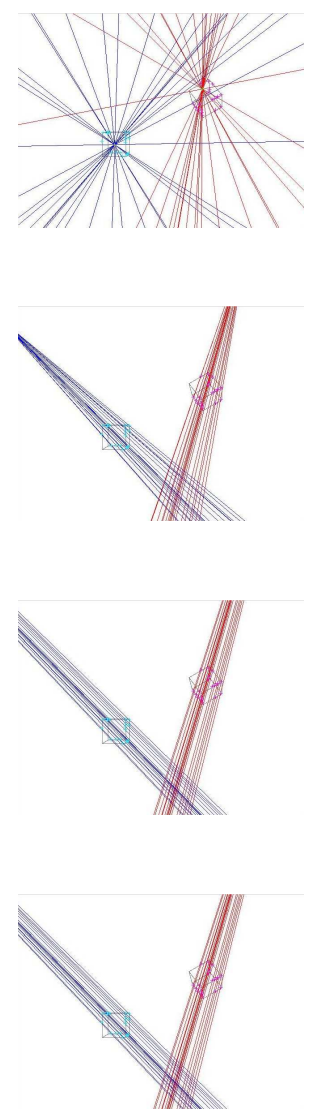

Figura 9.11: Resultados de simulação no caso de movimento planar. (a) Algoritmo de 7 Pontos Correspondentes. (b) Algoritmo de Busca Exaustiva. (c) Algoritmo de 8 Pontos Correspondentes Normalizado. (d) Algoritmo Linear Iterativo.

Tabela 9.4: Erro residual do Algoritmo de Busca Exaustiva na segunda montagem experimental $\left(\right.$ pixel $\left.^{2}\right)$.

\begin{tabular}{|c|r|r|r|r|}
\hline \multirow{2}{*}{ Imagens } & \multicolumn{2}{|c|}{ Conjunto A } & \multicolumn{2}{c|}{ Conjunto B } \\
\cline { 2 - 5 } & $\begin{array}{r}\text { Conjunto de } \\
\text { Entrada }\end{array}$ & $\begin{array}{r}\text { Conjunto } \\
\text { Complementar }\end{array}$ & $\begin{array}{r}\text { Conjunto de } \\
\text { Entrada }\end{array}$ & $\begin{array}{r}\text { Conjunto } \\
\text { Complementar }\end{array}$ \\
\hline \hline $01-02$ & 0.0286 & 0.4229 & 0.0144 & 0.0925 \\
\hline $02-03$ & 0.0556 & 0.0967 & 0.0666 & 0.1951 \\
\hline $04-05$ & 0.0262 & 0.1201 & 0.0450 & 0.1921 \\
\hline $01-04$ & 0.0372 & 0.1223 & 0.0638 & 0.0465 \\
\hline $01-05$ & 0.0880 & 0.2243 & 0.0504 & 0.1112 \\
\hline $02-04$ & 0.0197 & 0.1202 & 0.0228 & 0.0528 \\
\hline $02-05$ & 0.0209 & 0.0881 & 0.0062 & 0.0741 \\
\hline
\end{tabular}

Tabela 9.5: Erro residual do Algoritmo de 8 Pontos Correspondentes Normalizado na segunda montagem experimental ( pixel $\left.^{2}\right)$.

\begin{tabular}{|l|r|r|r|r|}
\hline \multirow{2}{*}{ Imagens } & \multicolumn{2}{|c|}{ Conjunto A } & \multicolumn{2}{c|}{ Conjunto B } \\
\cline { 2 - 5 } & $\begin{array}{r}\text { Conjunto de } \\
\text { Entrada }\end{array}$ & $\begin{array}{r}\text { Conjunto } \\
\text { Complementar }\end{array}$ & $\begin{array}{r}\text { Conjunto de } \\
\text { Entrada }\end{array}$ & $\begin{array}{r}\text { Conjunto } \\
\text { Complementar }\end{array}$ \\
\hline \hline $01-02$ & $1.2885 \mathrm{E}-5$ & $2.4579 \mathrm{E}-4$ & $2.0980 \mathrm{E}-5$ & $2.9946 \mathrm{E}-5$ \\
\hline $02-03$ & $3.2236 \mathrm{E}-5$ & $1.0429 \mathrm{E}-4$ & $1.3745 \mathrm{E}-5$ & $9.8883 \mathrm{E}-5$ \\
\hline $04-05$ & $1.9489 \mathrm{E}-5$ & $4.2778 \mathrm{E}-5$ & $2.2326 \mathrm{E}-5$ & $2.3772 \mathrm{E}-5$ \\
\hline $01-04$ & $1.0714 \mathrm{E}-5$ & $2.9684 \mathrm{E}-5$ & $4.8071 \mathrm{E}-5$ & $5.3492 \mathrm{E}-5$ \\
\hline $01-05$ & $4.0220 \mathrm{E}-6$ & $2.1606 \mathrm{E}-5$ & $7.4477 \mathrm{E}-6$ & $2.1924 \mathrm{E}-5$ \\
\hline $02-04$ & $9.2146 \mathrm{E}-6$ & $6.3630 \mathrm{E}-5$ & $1.3154 \mathrm{E}-5$ & $2.7106 \mathrm{E}-5$ \\
\hline $02-05$ & $1.4247 \mathrm{E}-5$ & $2.5172 \mathrm{E}-5$ & $9.0457 \mathrm{E}-6$ & $2.6824 \mathrm{E}-5$ \\
\hline
\end{tabular}



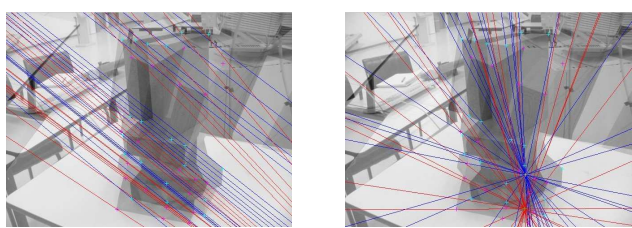

(a)
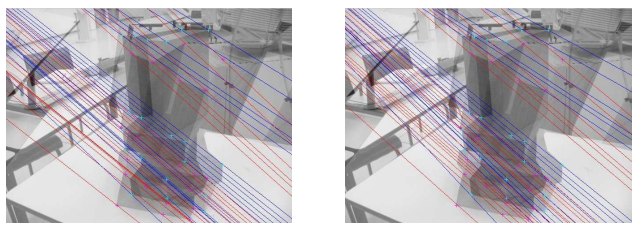

(b)
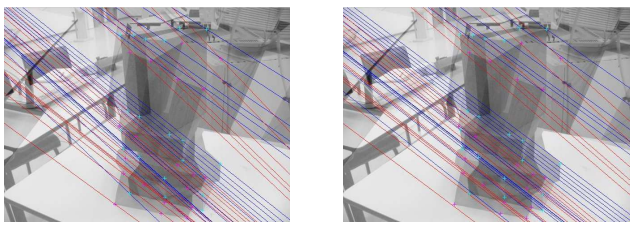

(c)
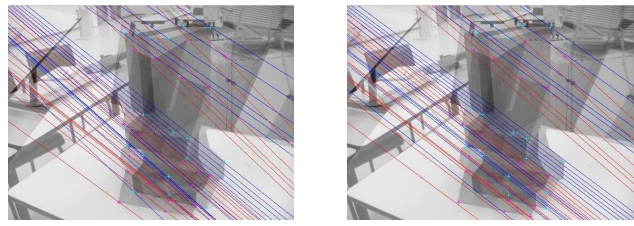
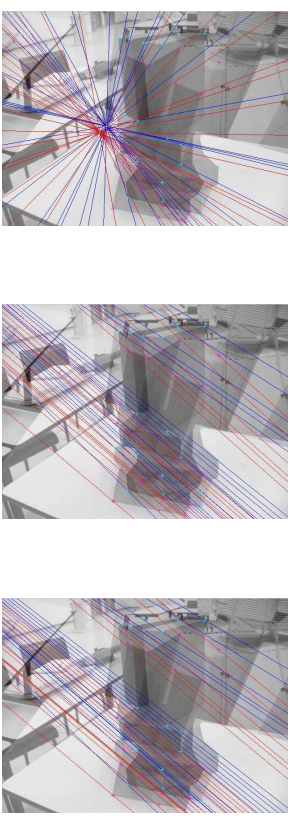

(d)

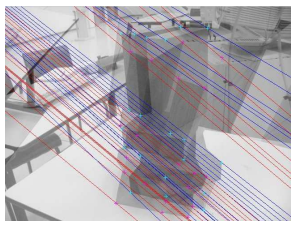

Figura 9.12: Resultados da primeira montagem experimental no caso de translação pura. (a) Algoritmo de 7 Pontos Correspondentes. (b) Algoritmo de Busca Exaustiva. (c) Algoritmo de 8 Pontos Correspondentes Normalizado. (d) Algoritmo Linear Iterativo.

Tabela 9.6: Erro residual do Algoritmo Linear Iterativo na segunda montagem experimental ( pixel $\left.^{2}\right)$.

\begin{tabular}{|l|r|r|r|r|}
\hline \multirow{2}{*}{ Imagens } & \multicolumn{2}{|c|}{ Conjunto A } & \multicolumn{2}{c|}{ Conjunto B } \\
\cline { 2 - 5 } & $\begin{array}{r}\text { Conjunto de } \\
\text { Entrada }\end{array}$ & $\begin{array}{r}\text { Conjunto } \\
\text { Complementar }\end{array}$ & $\begin{array}{r}\text { Conjunto de } \\
\text { Entrada }\end{array}$ & $\begin{array}{r}\text { Conjunto } \\
\text { Complementar }\end{array}$ \\
\hline \hline $01-02$ & 0.0016 & 0.0041 & $2.0980 \mathrm{E}-5$ & $2.9946 \mathrm{E}-5$ \\
\hline $02-03$ & $1.8324 \mathrm{E}-4$ & $5.0802 \mathrm{E}-4$ & $1.3745 \mathrm{E}-5$ & $9.8883 \mathrm{E}-5$ \\
\hline $04-05$ & $1.9489 \mathrm{E}-5$ & $4.2778 \mathrm{E}-5$ & $2.2326 \mathrm{E}-5$ & $2.3772 \mathrm{E}-5$ \\
\hline $01-04$ & $1.0714 \mathrm{E}-5$ & $2.9684 \mathrm{E}-5$ & $4.8071 \mathrm{E}-5$ & $5.3492 \mathrm{E}-5$ \\
\hline $01-05$ & $4.0220 \mathrm{E}-6$ & $2.1606 \mathrm{E}-5$ & $7.4477 \mathrm{E}-6$ & $2.1924 \mathrm{E}-5$ \\
\hline $02-04$ & $9.2146 \mathrm{E}-6$ & $6.3630 \mathrm{E}-5$ & $1.3154 \mathrm{E}-5$ & $2.7106 \mathrm{E}-5$ \\
\hline $02-05$ & $1.4247 \mathrm{E}-5$ & $2.5172 \mathrm{E}-5$ & $9.0457 \mathrm{E}-6$ & $2.6824 \mathrm{E}-5$ \\
\hline
\end{tabular}



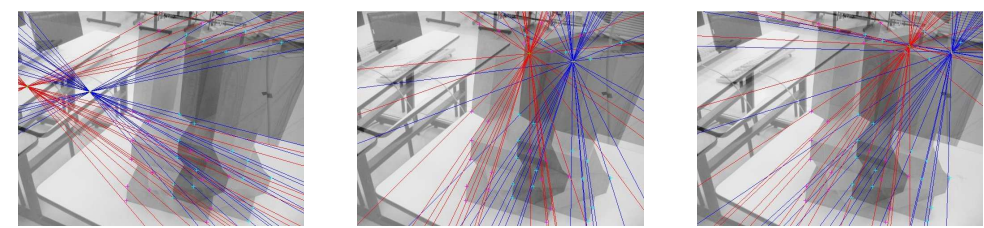

(a)
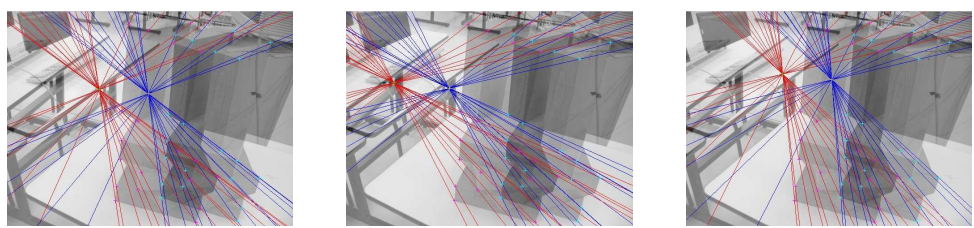

(b)
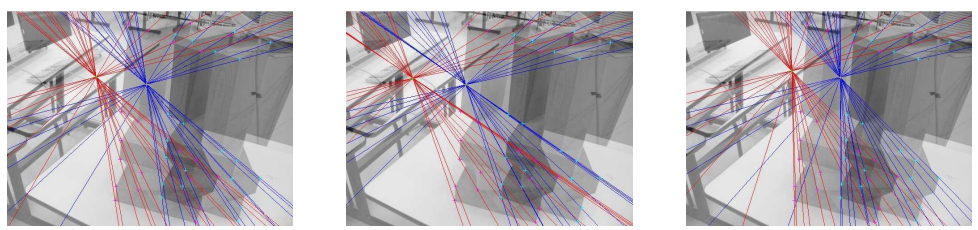

(c)
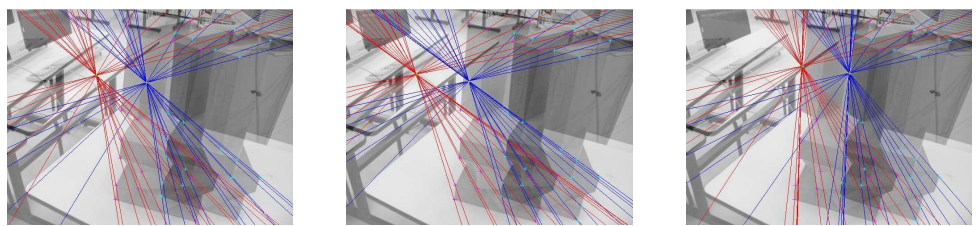

(d)

Figura 9.13: Resultados da primeira montagem experimental no caso de movimento geral. (a) Algoritmo de 7 Pontos Correspondentes. (b) Algoritmo de Busca Exaustiva. (c) Algoritmo de 8 Pontos Correspondentes Normalizado. (d) Algoritmo Linear Iterativo. 
Tabela 9.7: Erro residual do Algoritmos de 7 Pontos Correspondentes na terceira montagem experimental $\left(\right.$ pixel $\left.^{2}\right)$.

\begin{tabular}{|c|c|c|c|c|c|c|}
\hline \multirow[t]{2}{*}{ Imagens } & \multicolumn{2}{|c|}{$1^{\mathrm{a}}$ Solução } & \multicolumn{2}{|c|}{$2^{\mathrm{a}}$ Solução } & \multicolumn{2}{|c|}{$3^{\mathrm{a}}$ Solução } \\
\hline & $\begin{array}{r}\text { Conjunto de } \\
\text { Entrada }\end{array}$ & $\begin{array}{r}\text { Conjunto } \\
\text { Complementar }\end{array}$ & $\begin{array}{r}\text { Conjunto de } \\
\text { Entrada }\end{array}$ & $\begin{array}{r}\text { Conjunto } \\
\text { Complementar }\end{array}$ & $\begin{array}{r}\text { Conjunto de } \\
\text { Entrada }\end{array}$ & $\begin{array}{r}\text { Conjunto } \\
\text { Complementar }\end{array}$ \\
\hline $00-01$ & $2.6522 \mathrm{E}-24$ & 709.9579 & $1.3410 \mathrm{E}-24$ & $\overline{366.2674}$ & $8.1490 \mathrm{E}-26$ & 30.5092 \\
\hline $01-02$ & $5.0489 \mathrm{E}-29$ & 99.3778 & $7.4768 \mathrm{E}-28$ & 109.9501 & $4.2191 \mathrm{E}-28$ & 45.0941 \\
\hline $02-03$ & $6.8446 \mathrm{E}-27$ & 316.0635 & $2.5221 \mathrm{E}-27$ & 10.4675 & $2.3257 \mathrm{E}-27$ & 6.9854 \\
\hline $03-04$ & $8.2346 \mathrm{E}-27$ & 39.3512 & $5.1513 \mathrm{E}-27$ & 20.3046 & $2.3727 \mathrm{E}-28$ & 4.7088 \\
\hline $04-05$ & \multicolumn{4}{|c|}{ soluções inexistentes } & $9.3285 \mathrm{E}-27$ & 7.7051 \\
\hline $05-06$ & \multicolumn{4}{|c|}{ soluções inexistentes } & $1.5661 \mathrm{E}-26$ & 7.8397 \\
\hline $06-07$ & $2.9673 \mathrm{E}-25$ & $1.1135 \mathrm{E}+4$ & $1.3158 \mathrm{E}-25$ & 224.9943 & $1.1363 \mathrm{E}-25$ & 102.4944 \\
\hline $12-13$ & $1.4494 \mathrm{E}-28$ & 5.9372 & \multicolumn{4}{|c|}{ soluções inexistentes } \\
\hline $13-14$ & $1.5984 \mathrm{E}-24$ & $1.3088 \mathrm{E}+4$ & $1.0246 \mathrm{E}-26$ & 779.3397 & $4.8824 \mathrm{E}-26$ & 535.9915 \\
\hline $15-16$ & \multicolumn{4}{|c|}{ soluções inexistentes } & $7.0679 \mathrm{E}-27$ & 33.1471 \\
\hline $16-17$ & $1.0663 \mathrm{E}-27$ & 0.2187 & $5.2747 \mathrm{E}-27$ & 0.1207 & $1.9188 \mathrm{E}-26$ & 0.3634 \\
\hline
\end{tabular}

perimental, respectivamente para o Algoritmo de 7 Pontos Correspondentes, o Algoritmo de Busca Exaustiva, o Algoritmo de 8 Pontos Correspondentes Normalizado e o Algoritmo Linear Iterativo. Nestes casos as figuras também representam a superposição de cada par de imagens do conjunto de dados, com os pontos correspondentes e as retas epipolares obtidas através do respectivo algoritmo de determinação da matriz fundamental marcados sobre as mesmas.

O conjunto de dados da terceira montagem experimental é composto por uma seqüência de dezenove imagens numeradas 00 a 18, tomadas em pares seqüenciais. Em alguns pares de imagens não foi possível encontrar pares de pontos correspondentes suficientes para determinação da matriz fundamental (07-08, 10-11 e 17-18), e ainda outros foram descartados por não ter sido possível encontrar pontos de referência com projeções em ambas imagens suficientes para o cálculo das matrizes de projeção métricas (07-08, 08-09, 09-10, 11-12 e 14-15). Dessa forma, o conjunto de dados efetivamente utilizado nos cálculos é composto dos pares de imagens 00-01, 01-02, 02-03, 03-04, 04-05, 05-06, 06-07, 12-13, 13-14, $15-16$ e 16-17.

A Tabela 9.7 mostra o erro residual obtido com o Algoritmo de 7 Pontos Correspondentes para cada par de imagens do conjunto de dados da terceira montagem experimental. O erro residual foi calculado em relação aos pares de pontos correspondentes do subconjunto de entrada e com relação aos pares de pontos correspondentes do subconjunto complementar ao mesmo. Analogamente, as Tabelas 9.8, 9.9 e 9.10 apresentam o erro residual obtido respectivamente com o Algoritmo de Busca Exaustiva, o Algoritmo de 8 Pontos Correspondentes Normalizado e o Algoritmo Linear Iterativo, aplicados ao conjunto de dados da terceira montagem experimental. Em todos os casos, o valor do erro residual é dado em frações de pixel ao quadrado. 

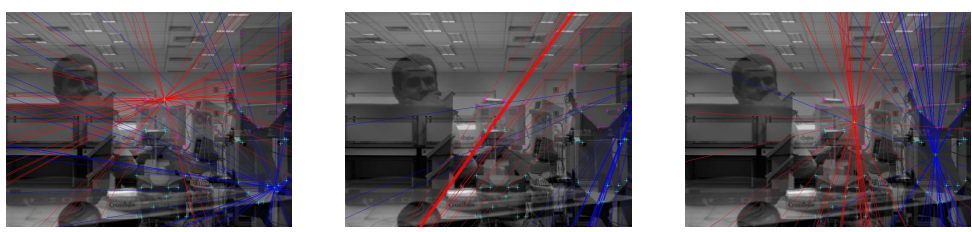

(a)
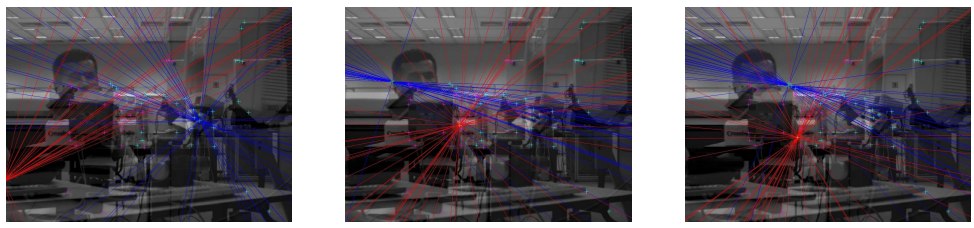

(b)
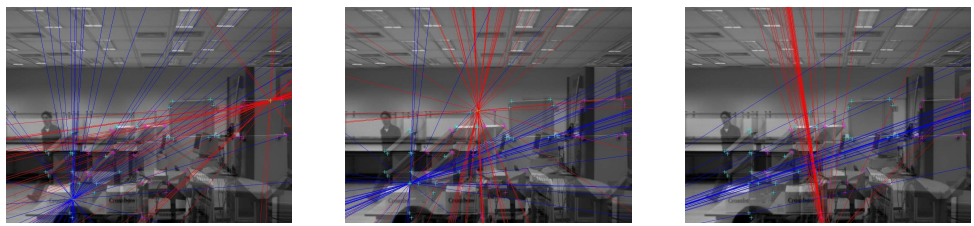

(c)
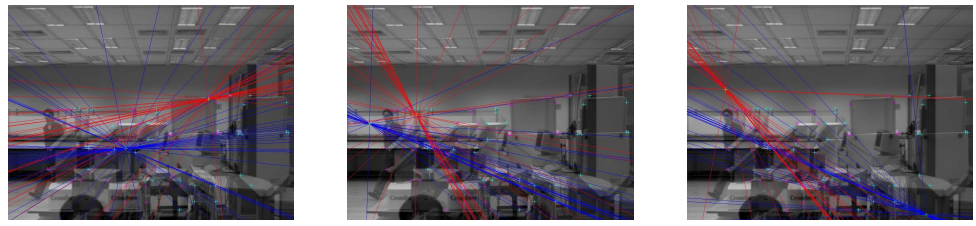

(d)

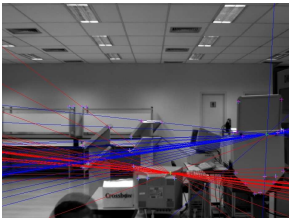

(e)
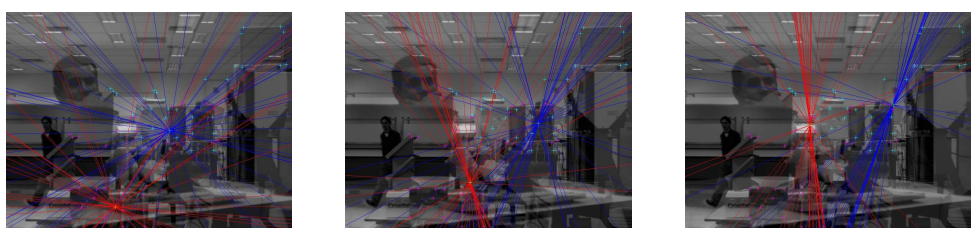

(f)
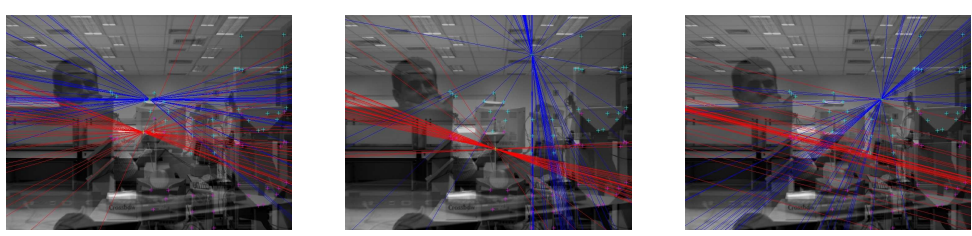

(g)

Figura 9.14: Resultados do Algoritmo de 7 Pontos Correspondentes na segunda montagem experimental. (a) 01-02. (b) 02-03 (c) 04-05 (d) 01-04. (e) 01-05. (f) 02-04. (g) 02-05. 

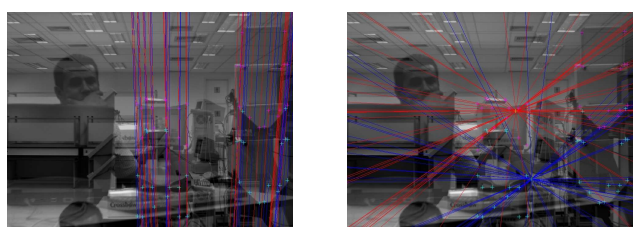

(a)
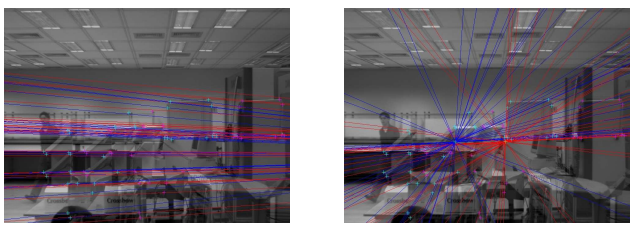

(c)
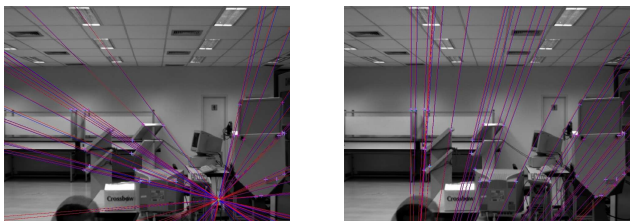

(e)

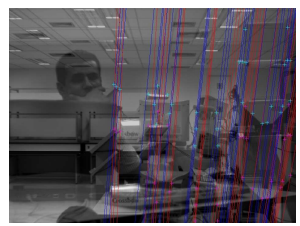

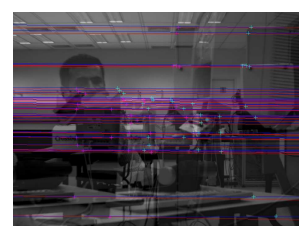

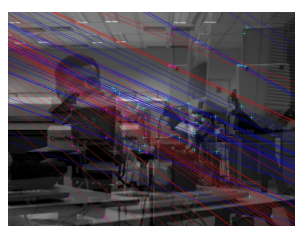

(b)
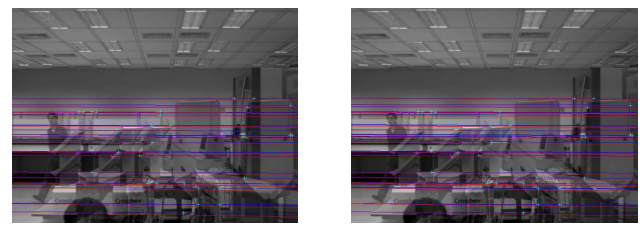

(d)
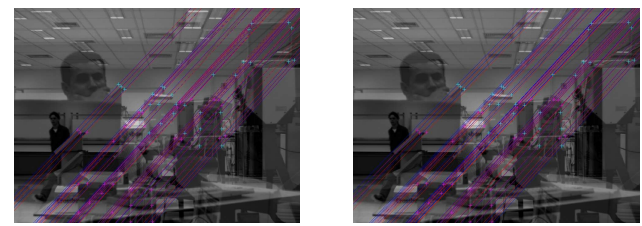

(f)

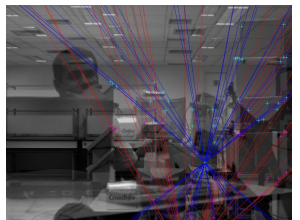

g)

Figura 9.15: Resultados do Algoritmo de Busca Exaustiva na segunda montagem experimental, para os subconjuntos A (esquerda) e B (direita). (a) 01-02. (b) 02-03 (c) 04-05 (d) 01-04. (e) 01-05. (f) 02-04. (g) 02-05.

Tabela 9.8: Erro residual do Algoritmo de Busca Exaustiva na terceira montagem experimental $\left(\right.$ pixel $\left.^{2}\right)$.

\begin{tabular}{|l|r|r|r|r|}
\hline \multirow{2}{*}{ Imagens } & \multicolumn{2}{|c|}{ Conjunto A } & \multicolumn{2}{c|}{ Conjunto B } \\
\cline { 2 - 5 } & $\begin{array}{r}\text { Conjunto de } \\
\text { Entrada }\end{array}$ & $\begin{array}{r}\text { Conjunto } \\
\text { Complementar }\end{array}$ & $\begin{array}{r}\text { Conjunto de } \\
\text { Entrada }\end{array}$ & $\begin{array}{r}\text { Conjunto } \\
\text { Complementar }\end{array}$ \\
\hline \hline $00-01$ & $1.2256 \mathrm{E}-4$ & $4.3285 \mathrm{E}-4$ & $6.5046 \mathrm{E}-5$ & $1.3810 \mathrm{E}-4$ \\
\hline $01-02$ & 0.0559 & 0.2782 & 0.0790 & 0.1163 \\
\hline $02-03$ & 0.0400 & 0.1251 & 0.0381 & 0.2289 \\
\hline $03-04$ & 0.0024 & 0.0102 & 0.0023 & 0.0156 \\
\hline $04-05$ & 0.0338 & 0.0546 & 0.0214 & 0.0715 \\
\hline $05-06$ & 0.0162 & 0.0364 & 0.0290 & 0.0281 \\
\hline $06-07$ & 0.0039 & 0.0032 & 0.0016 & 0.0053 \\
\hline $12-13$ & $6.1775 \mathrm{E}-5$ & $1.0824 \mathrm{E}-4$ & $6.1182 \mathrm{E}-5$ & $8.9825 \mathrm{E}-5$ \\
\hline $13-14$ & $4.0811 \mathrm{E}-4$ & 0.0047 & 0.0011 & 0.0014 \\
\hline $15-16$ & $3.0392 \mathrm{E}-4$ & 0.0013 & $2.2123 \mathrm{E}-4$ & $4.6878 \mathrm{E}-4$ \\
\hline $16-17$ & $4.1105 \mathrm{E}-6$ & $1.9457 \mathrm{E}-5$ & $5.7991 \mathrm{E}-6$ & $1.2255 \mathrm{E}-5$ \\
\hline
\end{tabular}



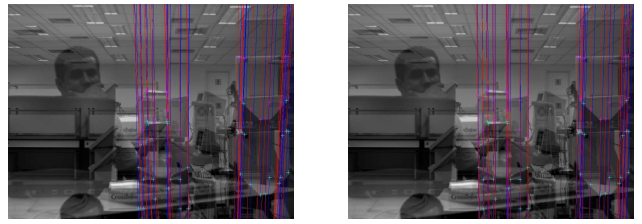

(a)
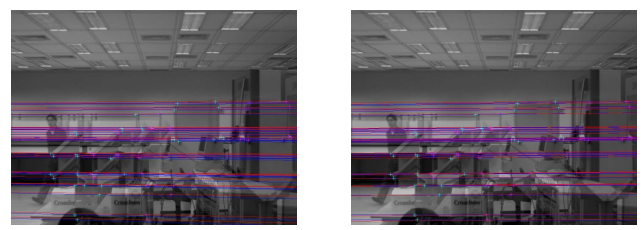

(c)
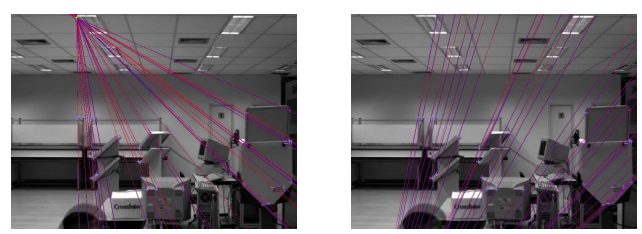

(e)

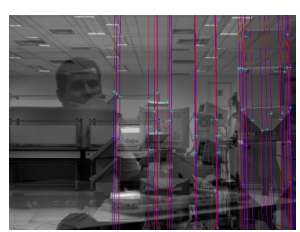

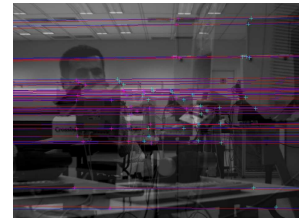

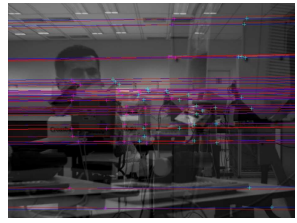

(b)
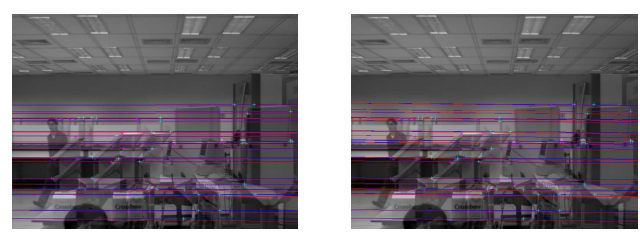

(d)
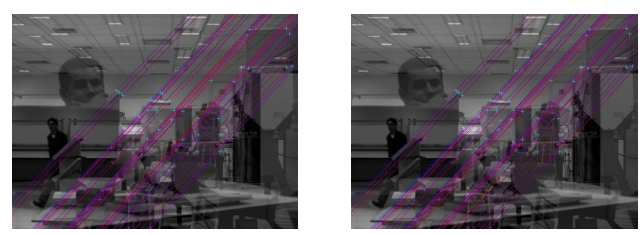

(f)

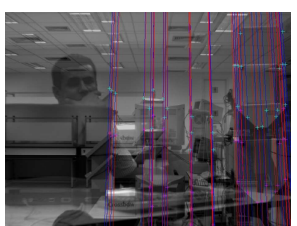

g)

Figura 9.16: Resultados do Algoritmo de 8 Pontos Correspondentes

Normalizado na segunda montagem experimental, para os subconjuntos A (esquerda) e B (direita). (a) 01-02. (b) 02-03 (c) 04-05 (d) 01-04. (e) 01-05. (f) 02-04. (g) 02-05.

Tabela 9.9: Erro residual do Algoritmo de 8 Pontos Correspondentes Normalizado na terceira montagem experimental $\left(\right.$ pixel $\left.^{2}\right)$.

\begin{tabular}{|l|r|r|r|r|}
\hline \multirow{2}{*}{ Imagens } & \multicolumn{2}{|c|}{ Conjunto A } & \multicolumn{2}{c|}{ Conjunto B } \\
\cline { 2 - 5 } & $\begin{array}{r}\text { Conjunto de } \\
\text { Entrada }\end{array}$ & $\begin{array}{r}\text { Conjunto } \\
\text { Complementar }\end{array}$ & $\begin{array}{r}\text { Conjunto de } \\
\text { Entrada }\end{array}$ & $\begin{array}{r}\text { Conjunto } \\
\text { Complementar }\end{array}$ \\
\hline \hline $00-01$ & $2.7144 \mathrm{E}-5$ & $2.6246 \mathrm{E}-5$ & $1.0694 \mathrm{E}-5$ & $2.0397 \mathrm{E}-5$ \\
\hline $01-02$ & $6.5995 \mathrm{E}-6$ & $2.8110 \mathrm{E}-5$ & $5.3539 \mathrm{E}-6$ & $1.4021 \mathrm{E}-5$ \\
\hline $02-03$ & $4.6881 \mathrm{E}-6$ & $1.0381 \mathrm{E}-5$ & $7.6585 \mathrm{E}-6$ & $1.5267 \mathrm{E}-5$ \\
\hline $03-04$ & $1.6329 \mathrm{E}-5$ & $5.0385 \mathrm{E}-5$ & $2.2334 \mathrm{E}-5$ & $6.8512 \mathrm{E}-5$ \\
\hline $04-05$ & $1.8854 \mathrm{E}-5$ & $1.9669 \mathrm{E}-5$ & $5.7517 \mathrm{E}-6$ & $2.5076 \mathrm{E}-5$ \\
\hline $05-06$ & $2.4342 \mathrm{E}-5$ & $4.3285 \mathrm{E}-5$ & $1.9148 \mathrm{E}-5$ & $3.5246 \mathrm{E}-5$ \\
\hline $06-07$ & $2.9010 \mathrm{E}-5$ & $3.0998 \mathrm{E}-5$ & $5.4128 \mathrm{E}-6$ & $1.9340 \mathrm{E}-5$ \\
\hline $12-13$ & $4.3020 \mathrm{E}-5$ & $7.4573 \mathrm{E}-5$ & $2.1778 \mathrm{E}-5$ & $2.8699 \mathrm{E}-5$ \\
\hline $13-14$ & $2.8316 \mathrm{E}-4$ & 0.0028 & $8.8034 \mathrm{E}-4$ & 0.0015 \\
\hline $15-16$ & $7.6590 \mathrm{E}-6$ & $3.4817 \mathrm{E}-5$ & $7.7099 \mathrm{E}-6$ & $1.6873 \mathrm{E}-5$ \\
\hline $16-17$ & $5.9543 \mathrm{E}-5$ & $3.8095 \mathrm{E}-4$ & $8.5814 \mathrm{E}-5$ & $2.1620 \mathrm{E}-4$ \\
\hline
\end{tabular}



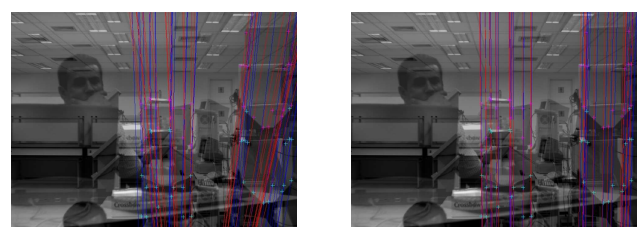

(a)
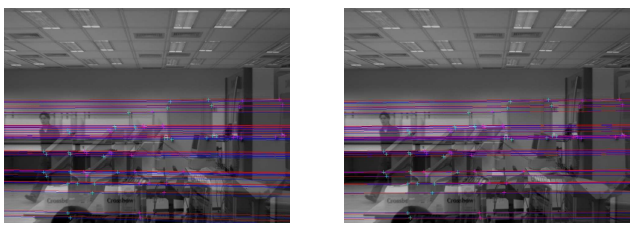

(c)
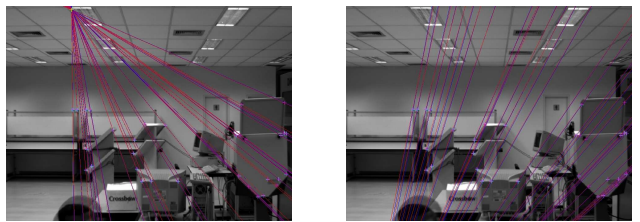

(e)

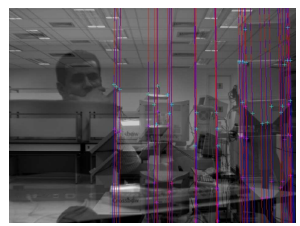

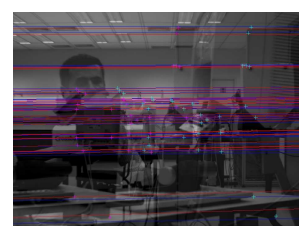

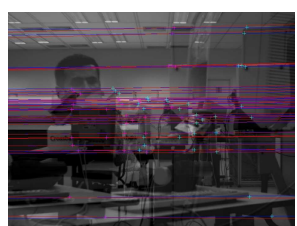

(b)
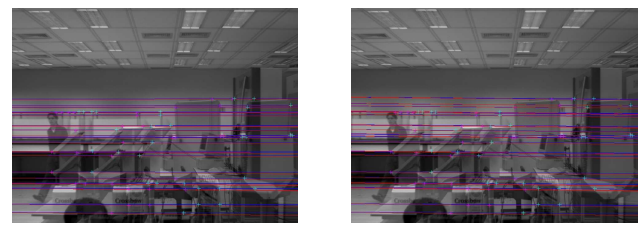

(d)
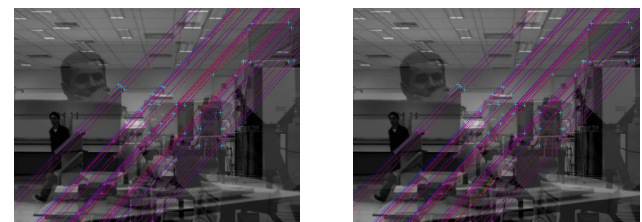

(f)

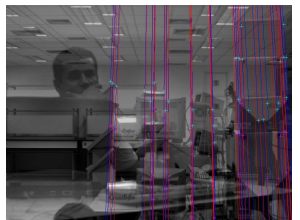

g)

Figura 9.17: Resultados do Algoritmo Linear Iterativo na segunda montagem experimental, para os subconjuntos A (esquerda) e B (direita). (a) 01-02. (b) 02-03 (c) 04-05 (d) 01-04. (e) 01-05. (f) 02-04. (g) 02-05.

Tabela 9.10: Erro residual do Algoritmo Linear Iterativo na terceira montagem experimental $\left(\right.$ pixel $\left.^{2}\right)$.

\begin{tabular}{|l|r|r|r|r|}
\hline \multirow{2}{*}{ Imagens } & \multicolumn{2}{|c|}{ Conjunto A } & \multicolumn{2}{c|}{ Conjunto B } \\
\cline { 2 - 5 } & $\begin{array}{r}\text { Conjunto de } \\
\text { Entrada }\end{array}$ & $\begin{array}{r}\text { Conjunto } \\
\text { Complementar }\end{array}$ & $\begin{array}{r}\text { Conjunto de } \\
\text { Entrada }\end{array}$ & $\begin{array}{r}\text { Conjunto } \\
\text { Complementar }\end{array}$ \\
\hline \hline $00-01$ & $3.8660 \mathrm{E}-5$ & $3.5822 \mathrm{E}-5$ & $1.0694 \mathrm{E}-5$ & $2.0397 \mathrm{E}-5$ \\
\hline $01-02$ & $6.5995 \mathrm{E}-6$ & $2.8110 \mathrm{E}-5$ & $5.3539 \mathrm{E}-6$ & $1.4021 \mathrm{E}-5$ \\
\hline $02-03$ & $4.6881 \mathrm{E}-6$ & $1.0381 \mathrm{E}-5$ & $7.6585 \mathrm{E}-6$ & $1.5267 \mathrm{E}-5$ \\
\hline $03-04$ & $1.6329 \mathrm{E}-5$ & $5.0385 \mathrm{E}-5$ & $2.2334 \mathrm{E}-5$ & $6.8512 \mathrm{E}-5$ \\
\hline $04-05$ & $1.8854 \mathrm{E}-5$ & $1.9669 \mathrm{E}-5$ & $5.7517 \mathrm{E}-6$ & $2.5076 \mathrm{E}-5$ \\
\hline $05-06$ & $2.4342 \mathrm{E}-5$ & $4.3285 \mathrm{E}-5$ & $1.9148 \mathrm{E}-5$ & $3.5246 \mathrm{E}-5$ \\
\hline $06-07$ & $2.9010 \mathrm{E}-5$ & $3.0998 \mathrm{E}-5$ & $5.4128 \mathrm{E}-6$ & $1.9340 \mathrm{E}-5$ \\
\hline $12-13$ & $4.3020 \mathrm{E}-5$ & $7.4573 \mathrm{E}-5$ & $2.1778 \mathrm{E}-5$ & $2.8699 \mathrm{E}-5$ \\
\hline $13-14$ & $9.9044 \mathrm{E}-4$ & 0.0011 & $8.8034 \mathrm{E}-4$ & 0.0015 \\
\hline $15-16$ & $7.6590 \mathrm{E}-6$ & $3.4817 \mathrm{E}-5$ & $7.7099 \mathrm{E}-6$ & $1.6873 \mathrm{E}-5$ \\
\hline $16-17$ & $5.9543 \mathrm{E}-5$ & $3.8095 \mathrm{E}-4$ & $8.5814 \mathrm{E}-5$ & $2.1620 \mathrm{E}-4$ \\
\hline
\end{tabular}


As Figuras 9.18 a 9.21 mostram os resultados dos algoritmos de cálculo da matriz fundamental aplicados ao conjunto de dados da terceira montagem experimental, respectivamente para o Algoritmo de 7 Pontos Correspondentes, o Algoritmo de Busca Exaustiva, o Algoritmo de 8 Pontos Correspondentes Normalizado e o Algoritmo Linear Iterativo. Nestes casos as figuras também representam a superposição de cada par de imagens do conjunto de dados, com os pontos correspondentes e as retas epipolares obtidas através do respectivo algoritmo de determinação da matriz fundamental marcados sobre as mesmas.

\subsubsection{Reconstrução Projetiva}

O cálculo das matrizes de projeção canônicas foi realizado diretamente a partir da matriz fundamental e do epipolo da segunda imagem conforme a equação (7.2), para cada um dos algoritmos de determinação da matriz fundamental e para cada par de imagens dos conjuntos de dados da segunda e da terceira montagens experimentais. A partir das matrizes de projeção canônica foram calculados por triangulação os pontos 3D referentes aos pares de pontos correspondentes dos subconjuntos complementares às entradas utilizadas no cálculo da matriz fundamental. O erro do processo de determinação das matrizes de projeção canônica é avaliado como o erro residual das projeções destes pontos 3D segundo a matriz fundamental utilizada para determinação das matrizes de projeção canônicas. Em todos os casos, o erro obtido é desprezível, resultado das operações em ponto flutuante no ambiente do MATLAB ${ }^{\circledR} 6.5$.

\subsubsection{Reconstrução Métrica}

O cálculo das matrizes de projeção métricas foi realizado a partir das matrizes de projeção canônicas e de cinco ou mais pontos de referência do espaço 3D, conforme o método descrito na Seção 7.4. Estas foram calculadas para cada par de matrizes de projeção canônicas obtido anteriormente - para cada algoritmo de determinação da matriz fundamental - e para cada par de imagens dos conjuntos de dados da segunda e da terceira montagem experimental. O erro introduzido por este processo foi analisado como a distância Euclidiana entre os pontos de referência originais utilizados na determinação das matrizes de projeção métricas e os pontos obtidos por triangulação dos pontos correspondentes nas imagens relativos à projeção dos mesmos em cada par de imagens. Estes pares de pontos correspondentes foram marcados independentemente do cálculo das matrizes de projeção, apenas com auxilio da interface gráfica desenvolvida em MATLAB ${ }^{\circledR}$ 

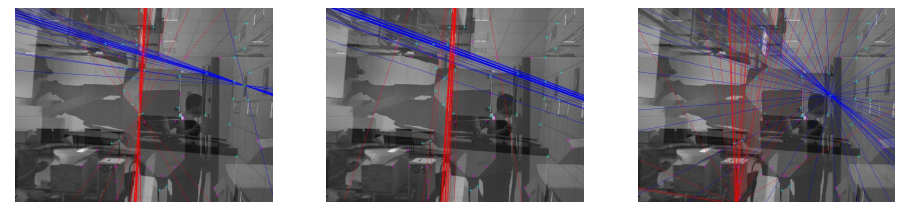

(a)
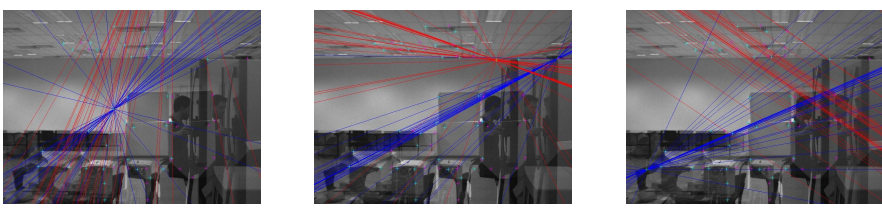

(b)
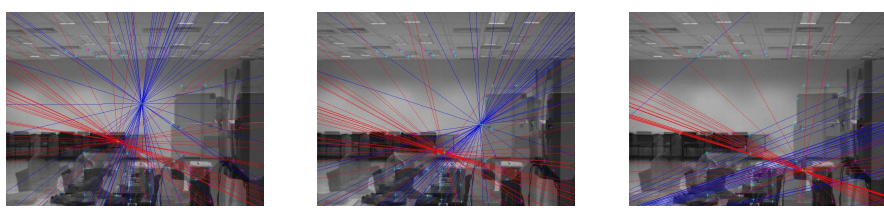

(c)
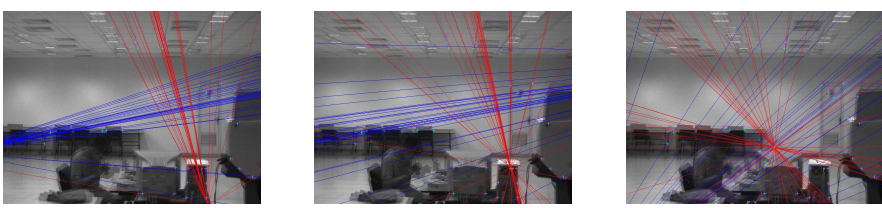

(d)

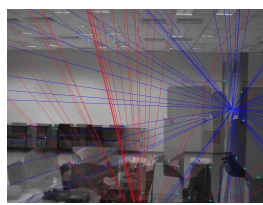

(e)

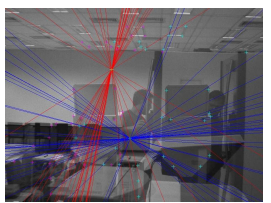

(f)

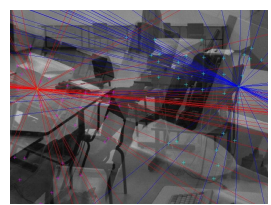

(g)

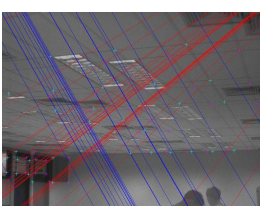

(h)
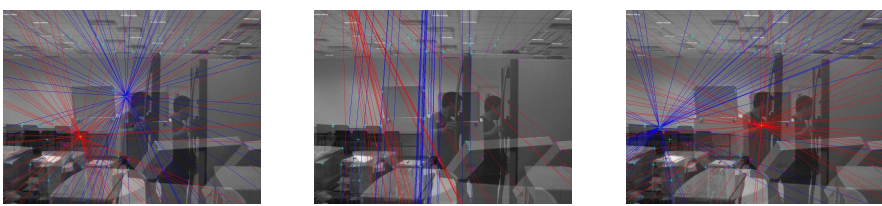

(i)
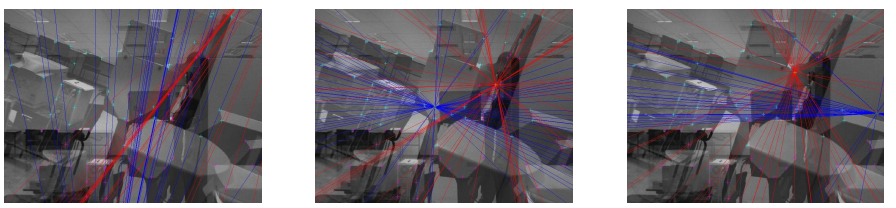

(j)
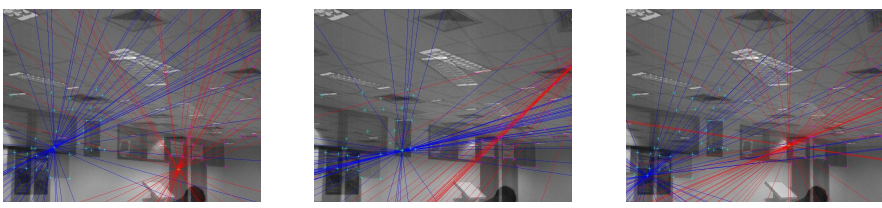

(k)

Figura 9.18: Resultados do Algoritmo de 7 Pontos Correspondentes na terceira montagem experimental. (a) 00-01. (b) 01-02. (c) 02-03. (d) 03-04. (e) 04-05. (f) 05-06. (g) 12-13. (h) 15-16. (i) 06-07. (j) 13-14. (k) 16-17. 

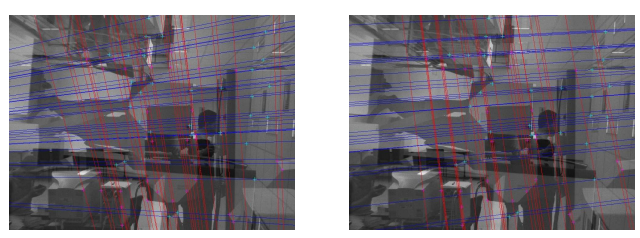

(a)
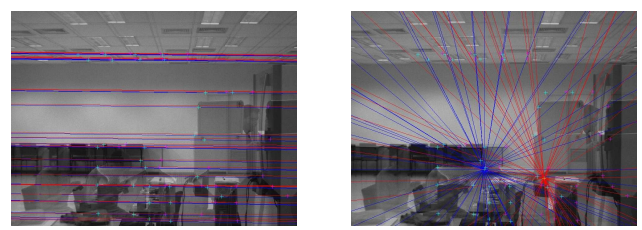

(c)
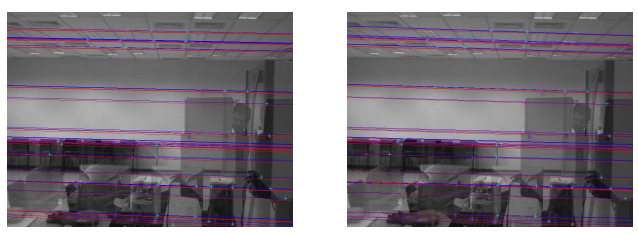

(e)
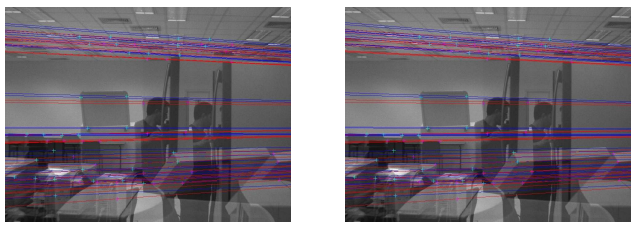

(g)
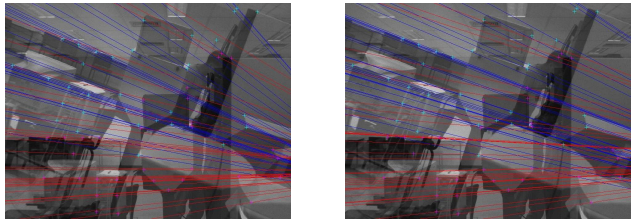

(i)

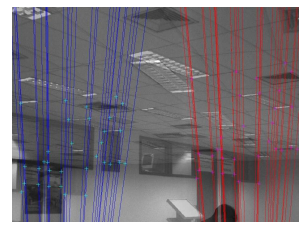

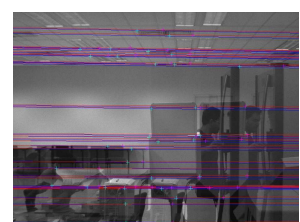

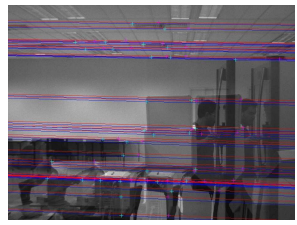

(b)
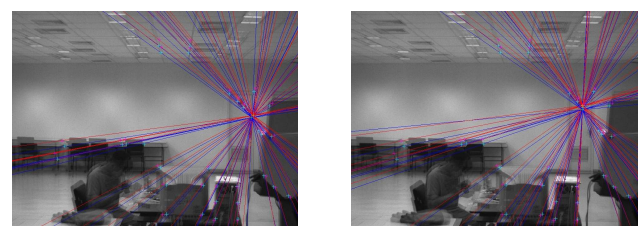

(d)
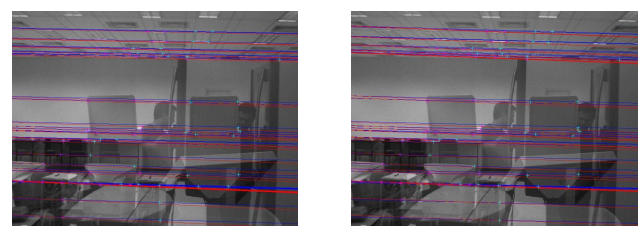

(f)
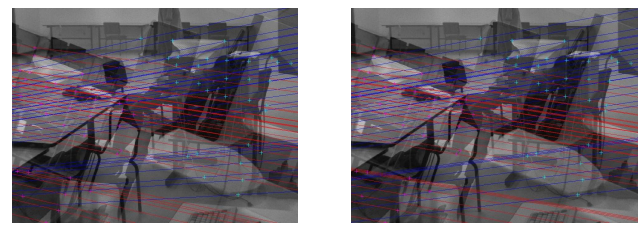

(h)
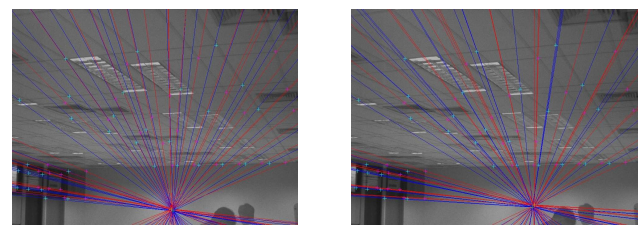

(j)

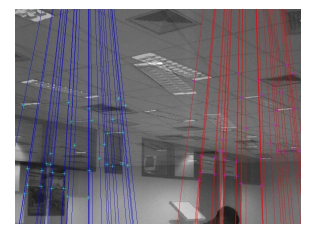

(k)

Figura 9.19: Resultado do Algoritmo de Busca Exaustiva na terceira montagem experimental, para os subconjuntos A (esquerda) e B (direita). (a) 00-01. (b) 01-02. (c) 02-03. (d) 03-04. (e) 04-05. (f) 05-06. (g) 06-07. (h) 12-13. (i) 13-14. (j) 15-16. (k) 16-17. 

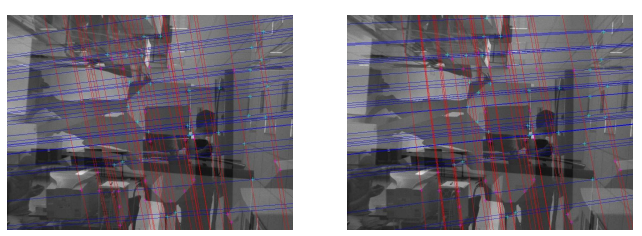

(a)
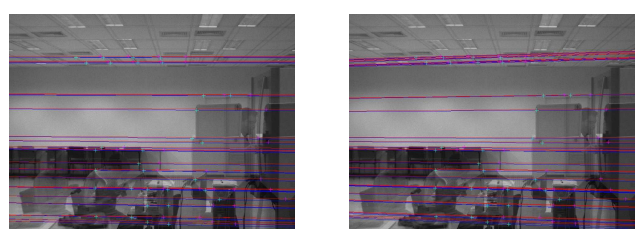

(c)
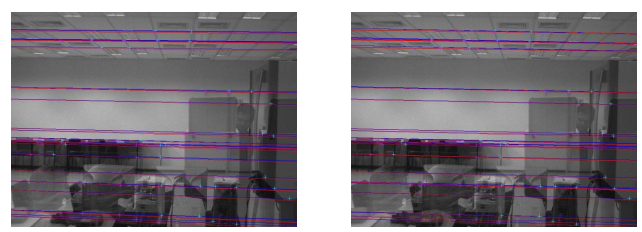

(e)
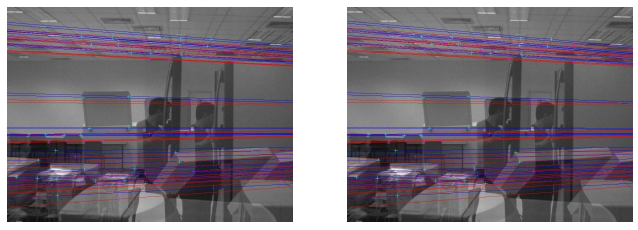

(g)
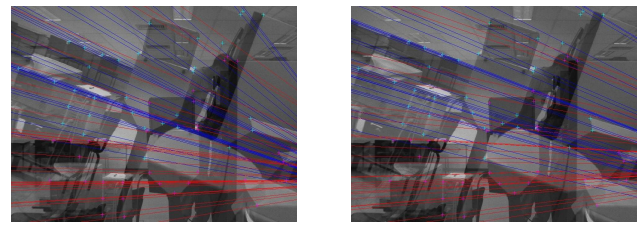

(i)

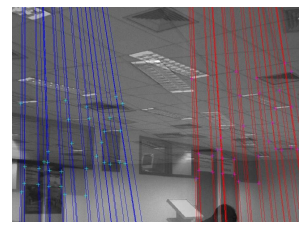

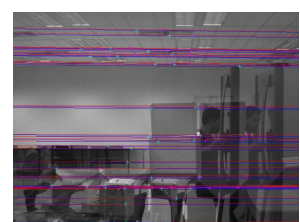

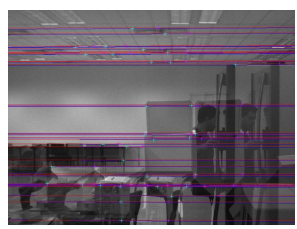

(b)
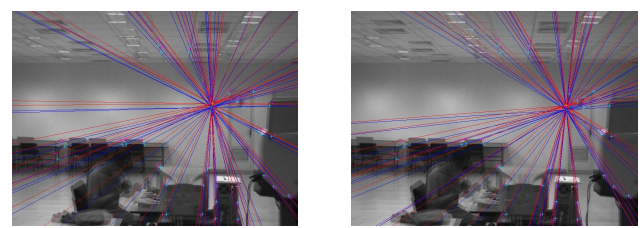

(d)
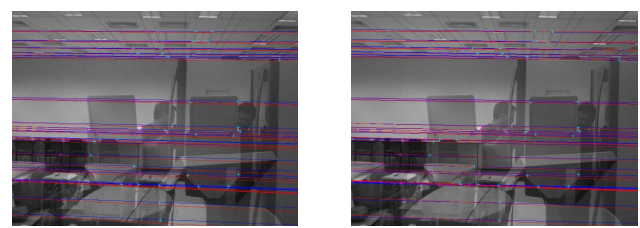

(f)
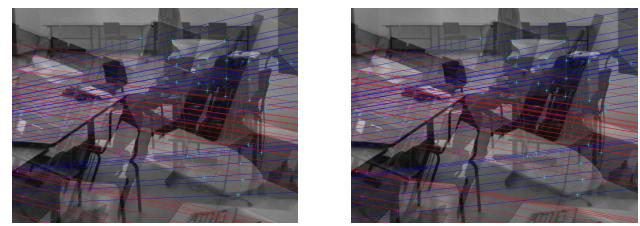

(h)
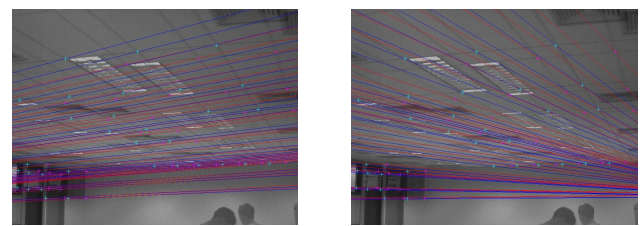

(j)

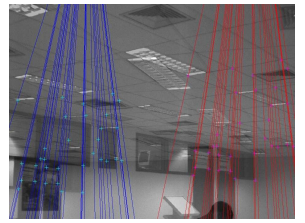

(k)

Figura 9.20: Resultado do Algoritmo de 8 Pontos Correspondentes

Normalizado na terceira montagem experimental, para os subconjuntos A (esquerda) e B (direita). (a) 00-01. (b) 01-02. (c) 02-03. (d) 03-04. (e) 04-05.

(f) 05-06. (g) 06-07. (h) 12-13. (i) 13-14. (j) 15-16. (k) 16-17. 

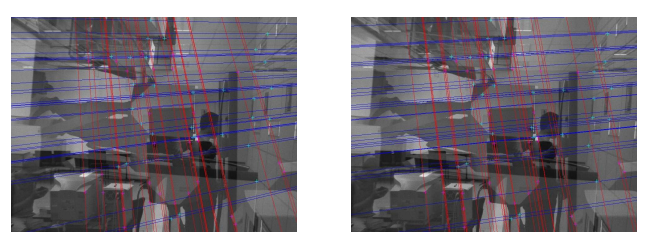

(a)
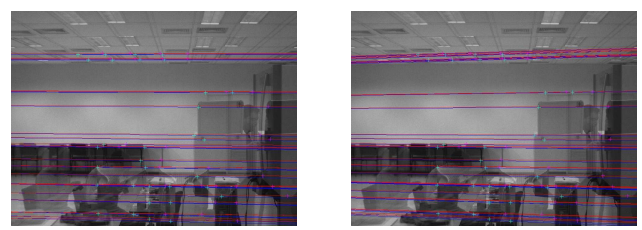

(c)
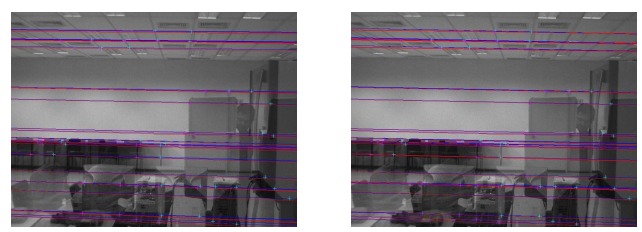

(e)
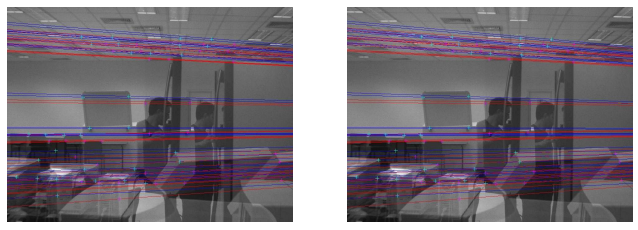

(g)
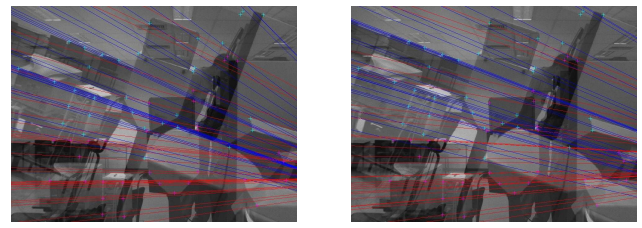

(i)

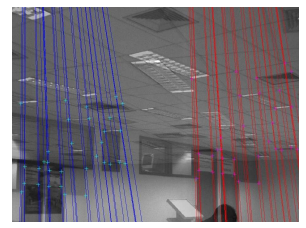

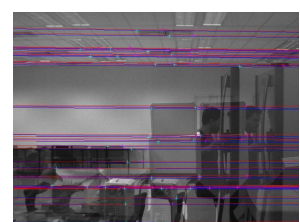

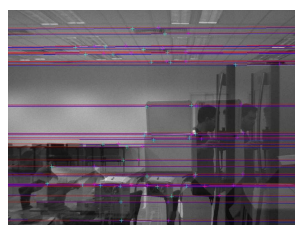

(b)
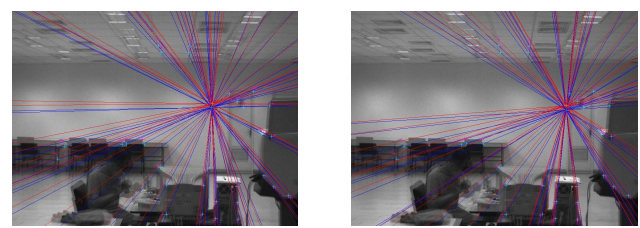

(d)
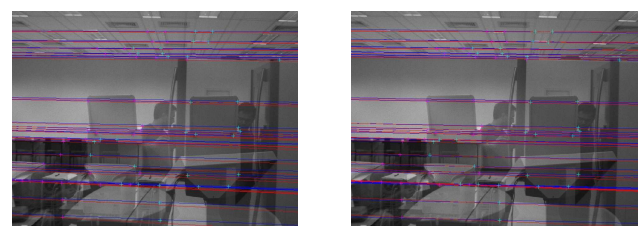

(f)
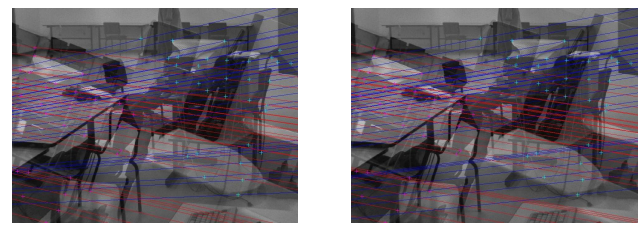

(h)
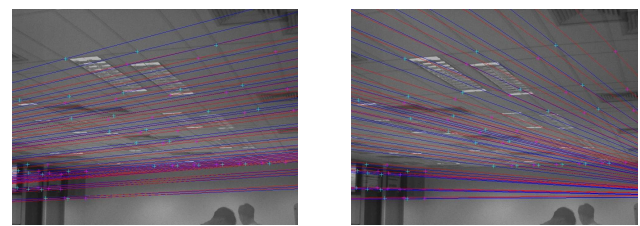

(j)

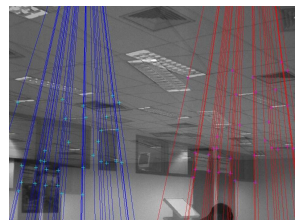

(k)

Figura 9.21: Resultado do Algoritmo Linear Iterativo na terceira montagem experimental, para os subconjuntos A (esquerda) e B (direita). (a) 00-01. (b) 01-02. (c) 02-03. (d) 03-04. (e) 04-05. (f) 05-06. (g) 06-07. (h) 12-13. (i) 13-14. (j) 15-16. (k) 16-17. 


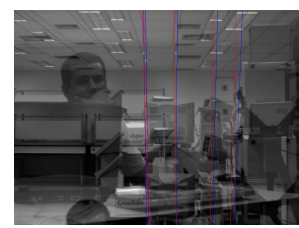

(a)

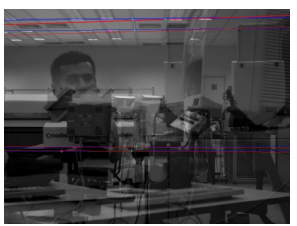

(b)

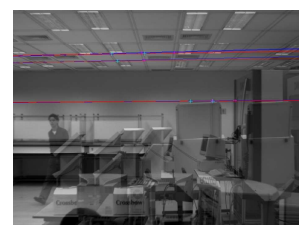

(c)

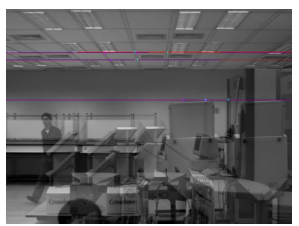

(d)

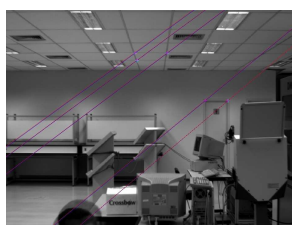

(e)

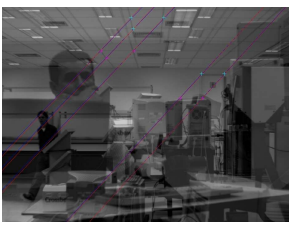

(f)

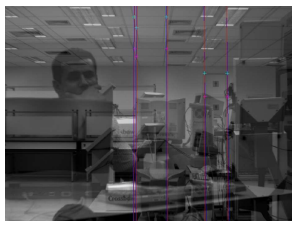

$(\mathrm{g})$

Figura 9.22: Pontos de referência 3D da segunda montagem experimental. (a) 01-02. (b) 02-03 (c) 04-05 (d) 01-04. (e) 01-05. (f) 02-04. (g) 02-05.

Tabela 9.11: Erro residual dos pontos de referência 3D da segunda montagem experimental $\left(\right.$ pixel $\left.^{2}\right)$.

\begin{tabular}{|c|c|c|c|c|c|c|c|c|c|}
\hline \multirow[t]{2}{*}{ Algoritmo } & \multicolumn{3}{|c|}{$\begin{array}{l}7 \text { Pontos } \\
\text { Correspondentes }\end{array}$} & \multicolumn{2}{|c|}{$\begin{array}{c}\text { Busca } \\
\text { Exaustiva }\end{array}$} & \multicolumn{2}{|c|}{8 Pontos } & \multicolumn{2}{|c|}{$\begin{array}{c}\text { Linear } \\
\text { Iterativo }\end{array}$} \\
\hline & $1^{\mathrm{a}}$ Sol. & $\frac{\text { responden }}{2^{\mathrm{a}} \text { Sol. }}$ & $3^{\mathrm{a}}$ Sol. & \begin{tabular}{c}
\multicolumn{2}{r}{ Exa } \\
Conj. A
\end{tabular} & 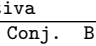 & $\begin{array}{l}\text { Corr. } \\
\text { Conj. }\end{array}$ & $\begin{array}{c}\text { malizado } \\
\text { Conj. B. B }\end{array}$ & \begin{tabular}{rr}
\multicolumn{2}{c}{ Ite } \\
Conj. A
\end{tabular} & tivo \\
\hline 01-02 & 83.1499 & 97.5005 & 7.4871 & 4.1361 & 6.7718 & 0.0016 & $2.6594 \mathrm{E}-4$ & 0.0165 & $2.6594 \mathrm{E}-4$ \\
\hline $02-03$ & 142.6159 & 438.2313 & 613.1824 & 0.8030 & 0.8964 & $1.8355 \mathrm{E}-4$ & $2.5978 \mathrm{E}-4$ & $3.6615 \mathrm{E}-4$ & $2.5978 \mathrm{E}-4$ \\
\hline $04-05$ & 30.4132 & 52.1045 & 29.5308 & 1.2349 & 9.8310 & $2.9666 \mathrm{E}-4$ & $8.3868 \mathrm{E}-5$ & $2.9666 \mathrm{E}-4$ & $8.3868 \mathrm{E}-5$ \\
\hline 01-04 & 952.5757 & 12.3005 & 15.2407 & 0.2212 & 0.4501 & $4.0376 \mathrm{E}-5$ & $2.5541 \mathrm{E}-4$ & $4.0376 \mathrm{E}-5$ & $2.5541 \mathrm{E}-4$ \\
\hline 01-05 & inex & entes & 33.8974 & 4.9926 & 0.6740 & $7.4633 \mathrm{E}-6$ & $5.0883 \mathrm{E}-5$ & $7.4633 \mathrm{E}-6$ & $5.0883 \mathrm{E}-5$ \\
\hline $02-04$ & 123.9605 & 24.8979 & 2.1932 & 0.8873 & 0.1823 & $1.8101 \mathrm{E}-4$ & $2.8402 \mathrm{E}-4$ & $1.8101 \mathrm{E}-4$ & $2.8402 \mathrm{E}-4$ \\
\hline $02-05$ & 504.4112 & 9.1941 & 70.9285 & 1.0695 & 4.9331 & $2.9847 \mathrm{E}-5$ & $2.5934 \mathrm{E}-4$ & $2.9847 \mathrm{E}-5$ & $2.5934 \mathrm{E}-4$ \\
\hline
\end{tabular}

6.5 .

As Figuras 9.22 e 9.23 mostram os pares de pontos correspondentes relativos aos pontos de referência 3D, respectivamente para cada par de imagens do conjunto de dados da segunda montagem experimental e do conjunto de dados da terceira montagem experimental. Em ambos os casos, as figuras são formadas pela superposição de cada par de imagens do conjunto de dados, com os pontos correspondentes relativos aos pontos de referência 3D e as retas epipolares marcados sobre as mesmas. As retas epipolares exibidas nestas imagens foram determinadas através do Algoritmo de 8 Pontos Correspondentes Normalizado aplicado ao conjunto completo de pares de pontos correspondentes como entrada. As Tabelas 9.11 e 9.12 mostram o erro residual destes pares de pontos correspondentes para cada algoritmo de determinação da matriz fundamental, respectivamente para o conjunto de dados da segunda e da terceira montagem experimental. O valor do erro residual é dado em frações de pixel ao quadrado.

A Tabela 9.13 mostra o erro da reconstrução dos pontos de referência 3D do conjunto de dados da segunda montagem experimental, para cada par de matrizes métricas de cada par de imagens, considerando cada algoritmo de determinação da matriz fundamental e cada subconjunto de pares de pontos correspondentes 


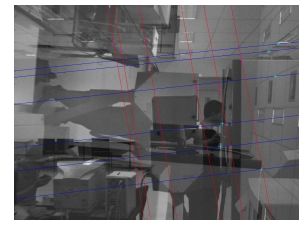

(a)

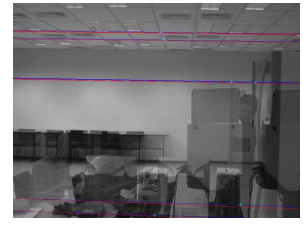

(e)

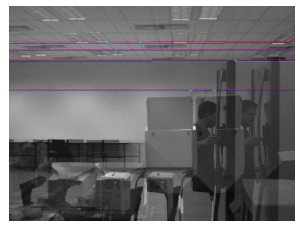

(b) 01-02

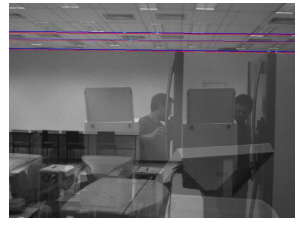

(f)

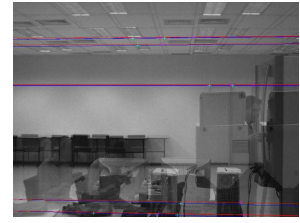

(c)

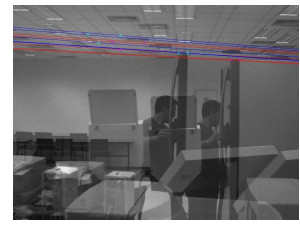

(g)

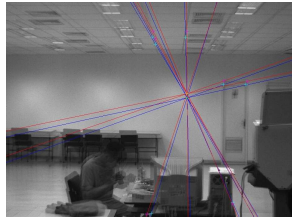

(d)

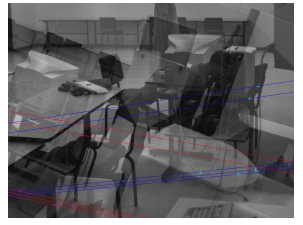

(h)

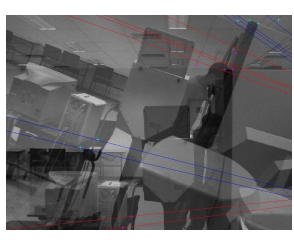

(i)

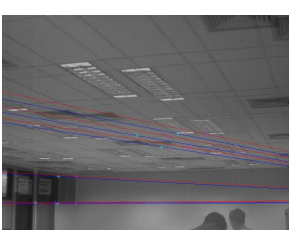

(j)

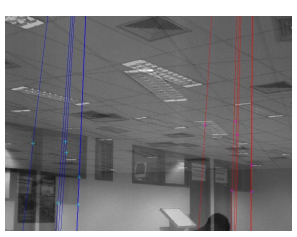

(k)

Figura 9.23: Pontos de referência $3 \mathrm{D}$ da terceira montagem experimental. (a) 00-01. (b) 01-02. (c) 02-03. (d) 03-04. (e) 04-05. (f) 05-06. (g) 06-07. (h) 12-13. (i) 13-14. (j) 15-16. (k) 16-17.

Tabela 9.12: Erro residual dos pontos de referência 3D na terceira montagem experimental $\left(\right.$ pixel $\left.^{2}\right)$.

\begin{tabular}{|c|c|c|c|c|c|c|c|c|c|}
\hline Algoritmo & \multicolumn{3}{|c|}{$\begin{array}{c}7 \text { Pontos } \\
\text { Correspondentes }\end{array}$} & \multicolumn{2}{|c|}{$\begin{array}{c}\text { Busca } \\
\text { Exaustiva }\end{array}$} & \multicolumn{2}{|c|}{8 Pontos } & \multicolumn{2}{|c|}{$\begin{array}{c}\text { Linear } \\
\text { Iterativo } \\
\end{array}$} \\
\hline & $1^{\mathrm{a}}$ Sol. & $2^{a}$ Sol. & $3^{\mathrm{a}}$ Sol. & Conj. A & Conj. & Conj. A & Conj. B & $\begin{array}{ll}\text { Conj. A } & \\
\end{array}$ & Conj. \\
\hline $00-01$ & 636.8358 & 303.1772 & 8.3734 & 0.0012 & $6.4286 \mathrm{E}-5$ & $5.1120 \mathrm{E}-6$ & $2.2972 \mathrm{E}-5$ & $1.1391 \mathrm{E}-4$ & $2.2972 \mathrm{E}-5$ \\
\hline $01-02$ & 4.9112 & 0.9782 & 1.0482 & 0.4221 & 0.2289 & $4.2077 \mathrm{E}-5$ & $2.4112 \mathrm{E}-5$ & $4.2077 \mathrm{E}-5$ & $2.4112 \mathrm{E}-5$ \\
\hline $02-03$ & 743.2200 & 30.4307 & 22.8847 & 0.1737 & 0.1972 & $1.0854 \mathrm{E}-5$ & $3.6075 \mathrm{E}-6$ & $1.0854 \mathrm{E}-5$ & $3.6075 \mathrm{E}-6$ \\
\hline $03-04$ & 3.3593 & 21.3460 & 20.6287 & 0.0037 & 0.0161 & $2.3118 \mathrm{E}-5$ & $9.3211 \mathrm{E}-5$ & $2.3118 \mathrm{E}-5$ & $9.3211 \mathrm{E}-5$ \\
\hline $04-05$ & \multicolumn{2}{|c|}{$\begin{array}{l}\text { inexistentes } \\
\text { inexistentes }\end{array}$} & 7.1617 & 0.1041 & 0.0715 & $1.5157 \mathrm{E}-5$ & $1.2295 \mathrm{E}-5$ & $1.5157 \mathrm{E}-5$ & $1.2295 \mathrm{E}-5$ \\
\hline $05-06$ & \multicolumn{2}{|c|}{ inexistentes } & 25.8121 & 0.0106 & 0.0508 & $1.2915 \mathrm{E}-4$ & $2.8384 \mathrm{E}-5$ & $1.2915 \mathrm{E}-4$ & $2.8384 \mathrm{E}-5$ \\
\hline $06-07$ & $.1326 \mathrm{E}+4$ & 262.2624 & 279.4831 & 0.0032 & $9.7102 \mathrm{E}-4$ & $3.8529 \mathrm{E}-5$ & $4.4647 \mathrm{E}-6$ & $3.8529 \mathrm{E}-5$ & $4.4647 \mathrm{E}-6$ \\
\hline $12-13$ & 61.0330 & \multicolumn{2}{|c|}{ inexistentes } & $1.5961 \mathrm{E}-4$ & $6.5311 \mathrm{E}-5$ & $6.5908 \mathrm{E}-5$ & $4.0255 \mathrm{E}-5$ & $6.5908 \mathrm{E}-5$ & $4.0255 \mathrm{E}-5$ \\
\hline $13-14$ & $1.0567 \mathrm{E}+3$ & 264.6594 & 291.7837 & 0.0423 & 0.0035 & 0.0262 & 0.0020 & 0.0038 & 0.0020 \\
\hline $15-16$ & \multicolumn{2}{|c|}{ inexistentes } & 9.0592 & $1.4419 \mathrm{E}-4$ & $2.9340 \mathrm{E}-4$ & $7.9875 \mathrm{E}-6$ & $4.8866 \mathrm{E}-6$ & $7.9875 \mathrm{E}-6$ & $4.8866 \mathrm{E}-6$ \\
\hline $16-17$ & 0.1586 & 0.1135 & 0.3462 & $1.4855 \mathrm{E}-5$ & $2.1807 \mathrm{E}-5$ & $3.6179 \mathrm{E}-4$ & $3.7657 \mathrm{E}-4$ & $3.6179 \mathrm{E}-4$ & $3.7657 \mathrm{E}-4$ \\
\hline
\end{tabular}


Tabela 9.13: Erro de reconstrução métrica dos pontos de referência 3D na segunda montagem experimental $\left(\mathrm{m}^{2}\right)$.

\begin{tabular}{|c|c|c|c|c|c|c|c|c|c|}
\hline \multirow[t]{2}{*}{ Algoritmo } & \multicolumn{3}{|c|}{$\begin{array}{c}7 \text { Pontos } \\
\text { Correspondentes }\end{array}$} & \multicolumn{2}{|c|}{$\begin{array}{c}\text { Busca } \\
\text { Exaustiva }\end{array}$} & \multicolumn{2}{|c|}{$\begin{array}{l}8 \text { Pontos } \\
. \quad \text { Normalizado }\end{array}$} & \multicolumn{2}{|c|}{$\begin{array}{l}\text { Linear } \\
\text { Iterativo }\end{array}$} \\
\hline & $1^{\mathrm{a}}$ Sol. & $2^{a}$ Sol. & $3^{a}$ Sol. & Conj. A & Conj. B & Conj. & Conj. B & Conj. & Conj. \\
\hline 01-02 & 4.8592 & 74.6164 & 697.4713 & 0.4068 & $4.4264 \mathrm{E}+3$ & 0.7240 & 0.2126 & 0.5234 & 0.2126 \\
\hline $02-03$ & 5.2771 & 9.5077 & 283.5084 & 0.0969 & $1.6765 \mathrm{E}+6$ & 0.0230 & 0.2418 & 4.0691 & 0.2418 \\
\hline $04-05$ & 365.4121 & 1.3186 & 24.8775 & 1.9773 & 70.1727 & 258.1689 & 5.3795 & 258.1689 & 5.3795 \\
\hline 01-04 & $1.4942 \mathrm{E}+3$ & 352.5723 & 157.8475 & 225.9808 & 193.0102 & 430.1303 & 15.5365 & 430.1303 & 15.5365 \\
\hline 01-05 & inexis & ntes & 98.7821 & $2.5599 \mathrm{E}-6$ & 12.6496 & 169.5327 & $1.1583 \mathrm{E}+3$ & 169.5327 & $1.1583 \mathrm{E}+3$ \\
\hline $02-04$ & 26.1999 & 14.3950 & 0.4980 & 565.2939 & 6.6100 & 473.2165 & $1.2566 \mathrm{E}+3$ & 473.2165 & $1.2566 \mathrm{E}+3$ \\
\hline $02-05$ & 1.6250 & 67.4296 & 1.6386 & $3.0195 \mathrm{E}+3$ & 0.6566 & 0.8671 & 158.8588 & 0.8671 & 158.8588 \\
\hline
\end{tabular}

Tabela 9.14: Erro de reconstrução métrica dos pontos de referência 3D na terceira montagem experimental $\left(\mathrm{m}^{2}\right)$.

\begin{tabular}{|c|c|c|c|c|c|c|c|c|c|}
\hline Algoritmo & \multicolumn{3}{|c|}{$\begin{array}{c}7 \text { Pontos } \\
\text { Correspondentes }\end{array}$} & \multicolumn{2}{|c|}{$\begin{array}{c}\text { Busca } \\
\text { Exaustiva }\end{array}$} & \multicolumn{2}{|c|}{$\begin{array}{ll} & \text { Pontos } \\
\text { Corr. } & \text { Normalizado }\end{array}$} & \multicolumn{2}{|c|}{$\begin{array}{c}\text { Linear } \\
\text { Iterativo }\end{array}$} \\
\hline & $1^{\mathrm{a}} \mathrm{Sol}$. & $2^{\mathrm{a}}$ Sol. & $3^{\mathrm{a}}$ Sol. & Conj & Conj. & Conj. & Conj. B & Conj. A & Conj. \\
\hline $00-01$ & 4.4176 & 2.3277 & $1.8441 \mathrm{E}+3$ & 0.0178 & 0.0061 & 0.0022 & 0.0101 & 0.0667 & 0.0101 \\
\hline $01-02$ & 0.3383 & 0.9450 & 4.0930 & 0.0657 & 0.0497 & 0.0742 & 0.0689 & 0.0742 & 0.0689 \\
\hline $02-03$ & 93.6027 & $5.8944 \mathrm{E}+3$ & 724.8240 & 0.4882 & $3.0196 \mathrm{E}+3$ & 0.7330 & 0.6245 & 0.7330 & 0.6245 \\
\hline $03-04$ & 150.2824 & $1.9206 \mathrm{E}+3$ & $9.4168 \mathrm{E}+4$ & 85.4312 & 112.1580 & 342.3615 & 8.9586 & 342.3615 & 8.9586 \\
\hline $04-05$ & \multicolumn{2}{|c|}{ inexistentes } & 123.9397 & 0.0321 & 0.0274 & 0.0476 & 0.0432 & 0.0476 & 0.0432 \\
\hline $05-06$ & \multicolumn{2}{|c|}{ inexistentes } & 1.4974 & 25.7513 & 124.4784 & 294.9285 & 16.8617 & 294.9285 & 16.8617 \\
\hline $06-07$ & 25.3175 & 84.5988 & 43.3415 & $2.3121 \mathrm{E}+3$ & 2.9322 & 2.7473 & 8.6054 & 2.7473 & 8.6054 \\
\hline $12-13$ & $1.54 \mathrm{E}-22$ & \multicolumn{2}{|c|}{ inexistentes } & $3.82 \mathrm{E}-22$ & $1.869 \mathrm{E}-22$ & $1.45 \mathrm{E}-23$ & $1.42 \mathrm{E}-22$ & $1.45 \mathrm{E}-23$ & $1.42 \mathrm{E}-22$ \\
\hline $13-14$ & 0.2716 & 1.4406 & 0.0671 & 0.1291 & 0.0377 & 0.1074 & 0.0393 & 0.1227 & 0.0393 \\
\hline $15-16$ & \multicolumn{2}{|c|}{ inexistentes } & 15.9105 & 0.9081 & 0.4886 & 0.0182 & 0.0316 & 0.0182 & 0.0316 \\
\hline $16-17$ & 1.2881 & 0.4751 & 1.2118 & 0.0587 & 0.0228 & 0.3669 & 0.0139 & 0.3669 & 0.0139 \\
\hline
\end{tabular}

usado como entrada dos mesmos. O erro consiste na somatória da distância Euclidiana entre os pontos de referência 3D originais e os pontos 3D reconstruídos pela triangulação dos pares de pontos correspondentes relativos aos mesmos, a partir das matrizes de projeção métricas, conforme descrito na Seção 7.5. Analogamente, Tabela 9.14 mostra o caso do conjunto de dados da terceira montagem experimental.

Além dos pontos de referência 3D utilizados para determinação das matrizes de projeção métricas, foram marcados pontos de teste 3D para o cálculo do desempenho de destas matrizes. Foram considerados pontos próximos aos pontos de referencia originais, que representassem planos definidos por estes mesmos pontos, e pontos quaisquer, alguns com apenas a distância relativa entre eles e parte das coordenadas conhecidas. Cada conjunto de pontos de teste 3D, de cada par de imagens dos conjuntos de dados da segunda e da terceira montagem experimental, foi dividido em dois blocos com o mesmo número de elementos, denominados $\mathrm{K}$ e L, sendo conhecidas as distâncias entre os pontos do bloco K e seu correspondente do bloco L. A partir das projeções destes pontos de teste 3D, marcadas em cada par de imagens, foram determinadas as reconstruções dos pontos originais, por triangulação a partir das matrizes de projeção métricas. Também foram calculadas as distâncias entre os pontos 3D reconstruídos, para comparação com as distâncias medidas anteriormente.

As Figuras 9.24 e 9.25 mostram os pares de pontos correspondentes relativos 


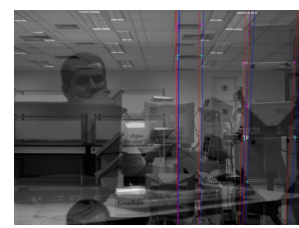

(a)

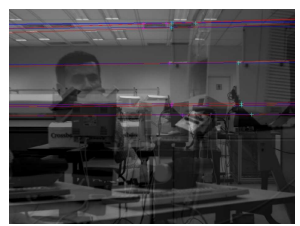

(b)

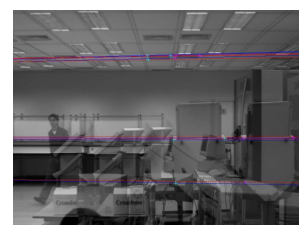

(c)

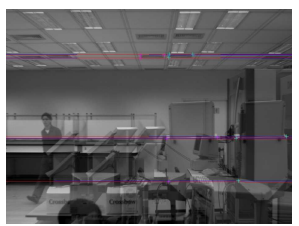

(d)

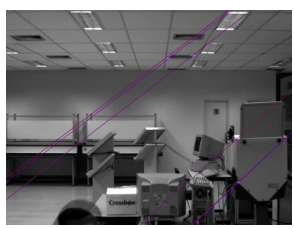

(e)

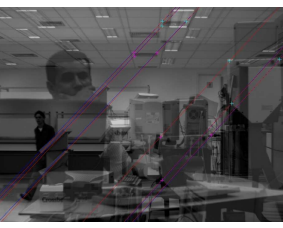

(f)

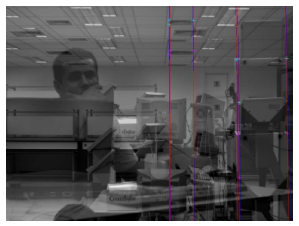

(g)

Figura 9.24: Pontos de teste 3D da segunda montagem experimental. (a) 01-02. (b) 02-03 (c) 04-05 (d) 01-04. (e) 01-05. (f) 02-04. (g) 02-05.

aos pontos de teste $3 \mathrm{D}$, respectivamente para cada par de imagens do conjunto de dados da segunda montagem experimental e do conjunto de dados da terceira montagem experimental. Em ambos os casos, as figuras são formadas pela superposição de cada par de imagens do conjunto de dados, com os pontos correspondentes relativos aos pontos de referência 3D e as retas epipolares marcados sobre as mesmas. As retas epipolares exibidas nestas imagens foram determinadas através do Algoritmo de 8 Pontos Correspondentes Normalizado aplicado ao conjunto completo de pares de pontos correspondentes como entrada.

As Tabelas 9.15 e 9.16 apresentam os resultados de erro da reconstrução dos pontos de teste $3 \mathrm{D}$, respectivamente para cada par de imagens do conjunto de dados da segunda e do conjunto de dados da terceira montagem experimental. Em ambos os casos, a reconstrução dos pontos de teste 3D foi calculada por triangulação a partir das matrizes de projeção métricas de cada par de imagens, para cada algoritmos de determinação da matriz fundamental. O erro consiste da distância Euclidiana quadrática entre o ponto de teste 3D original e o reconstruído, em metros ao quadrado. Os dados são apresentados em termos dos blocos $\mathrm{K}$ e L.

As Tabelas 9.17 e 9.18 mostram as distância entre os pontos de teste 3D do bloco $\mathrm{K}$ e os pontos correspondentes do bloco L, respectivamente para cada par de imagens do conjunto de dados da segunda montagem experimental e do conjunto de dados da terceira montagem experimental. Os dados correspondem às distâncias reais medidas, às distância entre os pontos reconstruídos por triangulação a partir das matrizes de projeção canônicas, e às distâncias entre os pontos reconstruídos por triangulação a partir das matrizes de projeção métricas. A distância real e a distância para a reconstrução a partir das matrizes de projeção 
Tabela 9.15: Erro de reconstrução métrica dos pontos de teste 3D na segunda montagem experimental $\left(\mathrm{m}^{2}\right)$.

\begin{tabular}{|c|c|c|c|c|c|c|c|c|c|c|}
\hline \multicolumn{2}{|c|}{ Algoritmo } & \multicolumn{3}{|c|}{$\begin{array}{c}7 \text { Pontos } \\
\text { Correspondentes }\end{array}$} & \multicolumn{2}{|c|}{$\begin{array}{c}\text { Busca } \\
\text { Exaustiva }\end{array}$} & \multicolumn{2}{|c|}{8 Pontos } & \multicolumn{2}{|c|}{$\begin{array}{c}\text { Linear } \\
\text { Iterativo }\end{array}$} \\
\hline & & $1^{\alpha}$ Sol. & $2^{\alpha}$ Sol. & $3^{\mathrm{a}}$ Sol. & Conj. A & Conj. & Conj. A & Conj. B & Conj. A & Conj. \\
\hline $01-02$ & $\overline{\mathrm{K}}$ & 13.5025 & 0.5643 & 5.5912 & 0.0854 & 0.6800 & 0.0850 & 0.0856 & 0.4077 & 0.0856 \\
\hline & & 13.5025 & 0.5643 & 5.5912 & 0.0854 & 0.6800 & 0.0850 & 0.0856 & 0.4077 & 0.0856 \\
\hline & & 86.8040 & 38.9881 & 67.4778 & 37.6295 & 22.8468 & 38.0241 & 38.5365 & 40.1573 & 38.5365 \\
\hline & & 86.8040 & 38.9881 & 67.4778 & 37.6295 & 22.8468 & 38.0241 & 38.5365 & 40.1573 & 38.5365 \\
\hline & $\mathrm{L}$ & 11.5437 & 1.4961 & 1.9284 & 0.3636 & 1.4644 & 0.1848 & 0.1176 & 0.8489 & 0.1176 \\
\hline & & 10.6883 & 0.9372 & 4.2728 & 0.5208 & 1.3388 & 0.4793 & 0.5293 & 0.5407 & 0.5293 \\
\hline & & 85.2654 & 83.4257 & 76.5675 & 38.6838 & 36.1478 & 39.0476 & 39.5351 & 41.1213 & 39.5351 \\
\hline & & 88.8769 & 38.3490 & 62.8355 & 38.3939 & 20.6852 & 38.7718 & 39.4030 & 40.9789 & 39.4030 \\
\hline $02-03$ & $\mathrm{~K}$ & 123.2346 & 19.8820 & 18.3109 & 482.9662 & 23.5269 & 105.3790 & 106.3997 & 152.8062 & 106.3997 \\
\hline & & 123.2346 & 19.8820 & 18.3109 & 482.9662 & 23.5269 & 105.3790 & 106.3997 & 152.8062 & 106.3997 \\
\hline & & 21.2671 & 12.3214 & 20.9302 & 260.4407 & 76.3498 & 267.8664 & 266.8481 & 252.5253 & 266.8481 \\
\hline & & 53.3023 & 1.6757 & 18.4480 & 333.2279 & 81.8156 & 346.1984 & 346.2515 & 310.4268 & 346.2515 \\
\hline & $\mathrm{L}$ & 1.9141 & 18.7240 & 9.8392 & $0.0000 \mathrm{e}+5$ & 14.3500 & $0.0000 \mathrm{e}+5$ & $0.0000 \mathrm{e}+5$ & $0.0002 \mathrm{e}+4$ & $0.0000 \mathrm{e}+5$ \\
\hline & & 135.2027 & 23.2930 & 21.4303 & $2.1268 \mathrm{e}+5$ & 19.1999 & $1.3820 \mathrm{e}+5$ & $3.1953 \mathrm{e}+5$ & $5.0290 \mathrm{e}+4$ & $3.1953 e+5$ \\
\hline & & 56.8713 & 1.9180 & 18.1020 & $0.0036 \mathrm{e}+5$ & 81.6716 & $0.0037 e+5$ & $0.0037 \mathrm{e}+5$ & $0.0333 \mathrm{e}+4$ & $0.0037 e+5$ \\
\hline & & 65.7833 & 2.4896 & 22.5126 & $0.0026 \mathrm{e}+5$ & 81.3767 & $0.0027 e+5$ & $0.0027 e+5$ & $0.0239 \mathrm{e}+4$ & $0.0027 e+5$ \\
\hline $04-05$ & $\mathrm{~K}$ & 0.8701 & 0.4474 & 0.7907 & 4.3482 & 0.3280 & 0.1369 & 0.4071 & 0.1369 & 0.4071 \\
\hline & & 0.8701 & 0.4474 & 0.7907 & 4.3482 & 0.3280 & 0.1369 & 0.4071 & 0.1369 & 0.4071 \\
\hline & & 43.0905 & 299.4321 & 25.8655 & 76.5433 & 36.7224 & 34.9618 & 33.5730 & 34.9618 & 33.5730 \\
\hline & & 43.0905 & 299.4321 & 25.8655 & 76.5433 & 36.7224 & 34.9618 & 33.5730 & 34.9618 & 33.5730 \\
\hline & $\mathrm{L}$ & 0.5181 & 0.6850 & 1.5594 & 9.4927 & 0.4190 & 3.9014 & 4.6378 & 3.9014 & 4.6378 \\
\hline & & 0.6299 & 0.2604 & 1.5755 & 5.2003 & 0.9440 & 1.1095 & 1.6653 & 1.1095 & 1.6653 \\
\hline & & 59.2170 & 505.9994 & 19.9177 & 77.2742 & 39.5264 & 36.2500 & 34.7546 & 36.2500 & 34.7546 \\
\hline & & 28.4422 & 6.8572 & 26.7662 & 76.6602 & 23.7479 & 35.3737 & 33.7799 & 35.3737 & 33.7799 \\
\hline 01-04 & $\mathrm{K}$ & $0.0078 \mathrm{e}+3$ & 0.5801 & 0.6935 & 0.4951 & 0.0856 & 0.1643 & 0.1797 & 0.1643 & 0.1797 \\
\hline & & $0.0078 e+3$ & 0.5801 & 0.6935 & 0.4951 & 0.0856 & 0.1643 & 0.1797 & 0.1643 & 0.1797 \\
\hline & & $2.7926 \mathrm{e}+3$ & 45.5289 & 27.3182 & 33.4990 & 34.7019 & 33.7768 & 34.7905 & 33.7768 & 34.7905 \\
\hline & & $2.7926 \mathrm{e}+3$ & 45.5289 & 27.3182 & 33.4990 & 34.7019 & 33.7768 & 34.7905 & 33.7768 & 34.7905 \\
\hline & $\mathrm{L}$ & 6.2508 & 0.2224 & 1.4746 & 4.7389 & 4.3471 & 6.6429 & 2.9852 & 6.6429 & 2.9852 \\
\hline & & 6.6989 & 0.3119 & 1.3682 & 0.5907 & 0.3219 & 0.3334 & 0.4226 & 0.3334 & 0.4226 \\
\hline & & 73.6362 & 50.8736 & 29.6058 & 34.6636 & 35.8818 & 34.9523 & 35.9572 & 34.9523 & 35.9572 \\
\hline & & 17.5678 & 41.5720 & 23.1408 & 33.7201 & 35.0737 & 34.0283 & 35.1386 & 34.0283 & 35.1386 \\
\hline 01-05 & $\mathrm{K}$ & inexi & ntes & 7.3867 & 0.3993 & 0.6511 & 0.0459 & 0.6076 & 0.0459 & 0.6076 \\
\hline & & & & 7.3867 & 0.3993 & 0.6511 & 0.0459 & 0.6076 & 0.0459 & 0.6076 \\
\hline & & & & 56.4652 & 57.8506 & 29.9834 & 139.1092 & 28.0900 & 139.1092 & 28.0900 \\
\hline & & & & 56.4652 & 57.8506 & 29.9834 & 139.1092 & 28.0900 & 139.1092 & 28.0900 \\
\hline & $\mathrm{L}$ & inexi & ntes & 7.5742 & 1.1331 & 1.4973 & 0.1203 & 1.0272 & 0.1203 & 1.0272 \\
\hline & & & & 5.2370 & 0.5710 & 1.2264 & 0.1888 & 1.2836 & 0.1888 & 1.2836 \\
\hline & & & & 69.3744 & 58.3823 & 32.7285 & 103.1686 & 34.3811 & 103.1686 & 34.3811 \\
\hline & & & & 64.8767 & 42.9103 & 25.9007 & 392.6429 & 41.9110 & 392.6429 & 41.9110 \\
\hline 02-04 & $\mathrm{K}$ & 4.9400 & 9.5245 & 0.7889 & 0.7526 & 0.7703 & 0.8014 & 0.7399 & 0.8014 & 0.7399 \\
\hline & & 4.9400 & 9.5245 & 0.7889 & 0.7526 & 0.7703 & 0.8014 & 0.7399 & 0.8014 & 0.7399 \\
\hline & & 44.8174 & 76.2129 & 59.5161 & 40.4734 & 41.0264 & 40.0983 & 40.0365 & 40.0983 & 40.0365 \\
\hline & & 44.8174 & 76.2129 & 59.5161 & 40.4734 & 41.0264 & 40.0983 & 40.0365 & 40.0983 & 40.0365 \\
\hline & $\mathrm{L}$ & 3.8940 & 9.4282 & 0.9180 & 0.4754 & 0.4037 & 0.5018 & 0.4599 & 0.5018 & 0.4599 \\
\hline & & 3.4777 & 7.0487 & 0.5576 & 2.2875 & 2.1910 & 2.5980 & 2.4024 & 2.5980 & 2.4024 \\
\hline & & 79.8556 & 79.7398 & 55.8482 & 41.3323 & 41.8742 & 41.0098 & 40.9551 & 41.0098 & 40.9551 \\
\hline & & 62.1530 & 77.5974 & 15.3790 & 41.5779 & 42.2040 & 41.1921 & 41.1082 & 41.1921 & 41.1082 \\
\hline $02-05$ & $\mathrm{~K}$ & 1.9468 & 8.8610 & 0.0816 & 0.0825 & 1.1442 & 0.1111 & 0.1032 & 0.1111 & 0.1032 \\
\hline & & 1.9468 & 8.8610 & 0.0816 & 0.0825 & 1.1442 & 0.1111 & 0.1032 & 0.1111 & 0.1032 \\
\hline & & 286.8912 & 85.6537 & 902.3665 & 36.9205 & 45.0088 & 38.2513 & 37.5344 & 38.2513 & 37.5344 \\
\hline & & 286.8912 & 85.6537 & 902.3665 & 36.9205 & 45.0088 & 38.2513 & 37.5344 & 38.2513 & 37.5344 \\
\hline & $\mathrm{L}$ & 0.9741 & 87.5925 & 0.2059 & 1.0056 & 1.9857 & 0.4898 & 0.4603 & 0.4898 & 0.4603 \\
\hline & & 1.1954 & 7.0185 & 0.2137 & 0.2438 & 0.6375 & 0.7392 & 0.6370 & 0.7392 & 0.6370 \\
\hline & & 125.7607 & 86.6232 & 71.4963 & 37.9819 & 46.3878 & 39.2303 & 38.5475 & 39.2303 & 38.5475 \\
\hline & & 57.7396 & 78.0931 & 45.3324 & 37.5256 & 44.1535 & 39.0993 & 38.3433 & 39.0993 & 38.3433 \\
\hline
\end{tabular}


Tabela 9.16: Erro da reconstrução métrica dos pontos de teste 3D na terceira montagem experimental $\left(\mathrm{m}^{2}\right)$.

\begin{tabular}{|c|c|c|}
\hline Algori & & \\
\hline & & $1^{\mathrm{a}}$ Sol. \\
\hline $00-01$ & $\mathrm{~K}$ & 0.0364 \\
\hline & & 0.0364 \\
\hline & & 53.1968 \\
\hline & & 53.1968 \\
\hline & $\mathrm{L}$ & 0.6738 \\
\hline & & 0.0968 \\
\hline & & 77.5930 \\
\hline & & 5.2397 \\
\hline 01-02 & K & 0.1013 \\
\hline & & 0.1013 \\
\hline & & 95.8776 \\
\hline & & 95.8776 \\
\hline & & 10.1904 \\
\hline & $\mathrm{L}$ & 0.8691 \\
\hline & & 0.0800 \\
\hline & & 91.1419 \\
\hline & & 96.3721 \\
\hline & & 23.3879 \\
\hline $02-03$ & K & 0.0558 \\
\hline & & 0.0558 \\
\hline & & 3.8327 \\
\hline & & 1.7311 \\
\hline & & 9.4231 \\
\hline & $\mathrm{L}$ & 63.7821 \\
\hline & & 1.7127 \\
\hline & & 1.5093 \\
\hline & & 2.0469 \\
\hline & & 397.0154 \\
\hline $03-04$ & K & 0.8272 \\
\hline & & 0.8272 \\
\hline & & 3.7596 \\
\hline & & 1.7794 \\
\hline & & 2.0175 \\
\hline & $\overline{\mathrm{L}}$ & $8.5486 \mathrm{e}+3$ \\
\hline & & $0.0001 \mathrm{e}+3$ \\
\hline & & $0.0018 \mathrm{e}+3$ \\
\hline & & $0.0016 e+3$ \\
\hline & & $0.0378 e+3$ \\
\hline $04-05$ & K & inexis \\
\hline & & \\
\hline & & \\
\hline & & \\
\hline & & \\
\hline & $\mathrm{L}$ & inexis \\
\hline & & \\
\hline & & \\
\hline & & \\
\hline & & \\
\hline $05-06$ & K & inexis \\
\hline & & \\
\hline & & \\
\hline & & \\
\hline & & \\
\hline & $\overline{\mathrm{L}}$ & inexis \\
\hline & & \\
\hline & & \\
\hline & & \\
\hline & & \\
\hline $06-07$ & K & 31.6115 \\
\hline & & 31.6115 \\
\hline & & 0.4137 \\
\hline & & 0.4137 \\
\hline & & 83.8444 \\
\hline & $\mathrm{L}$ & 30.9286 \\
\hline & & 36.7317 \\
\hline & & 0.0565 \\
\hline & & 0.4059 \\
\hline & & 81.6094 \\
\hline $12-13$ & $\mathrm{~K}$ & 31.2477 \\
\hline & & 13.7546 \\
\hline & $\mathrm{L}$ & 33.6148 \\
\hline & & 11.9291 \\
\hline $13-14$ & $\mathrm{~K}$ & $0.0003 e+3$ \\
\hline & & $0.0003 e+3$ \\
\hline & & $0.0106 \mathrm{e}+3$ \\
\hline & & $1.5466 \mathrm{e}+3$ \\
\hline & $\overline{\mathrm{L}}$ & $1.3826 \mathrm{e}+5$ \\
\hline & & $0.0000 \mathrm{e}+5$ \\
\hline & & $0.0001 \mathrm{e}+5$ \\
\hline & & $0.0155 e+5$ \\
\hline $15-16$ & $\mathrm{~K}$ & inexis \\
\hline & & \\
\hline & & \\
\hline & L & inexis \\
\hline & & \\
\hline & & \\
\hline $16-17$ & K & 0.9679 \\
\hline & & 0.9679 \\
\hline & & 0.5801 \\
\hline & & 21.4724 \\
\hline & $\bar{L}$ & 0.0638 \\
\hline & & 0.3162 \\
\hline & & 0.8576 \\
\hline & & 3.0706 \\
\hline
\end{tabular}




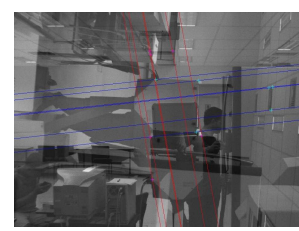

(a)

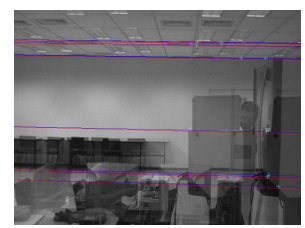

(e)

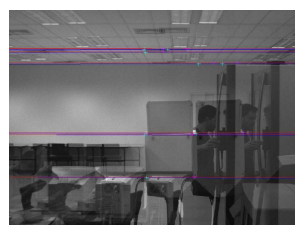

(b)

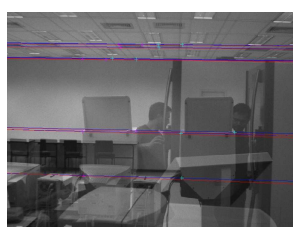

(f)

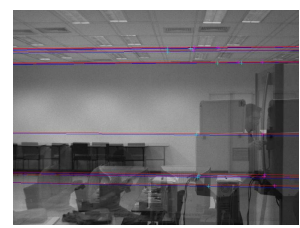

(c)

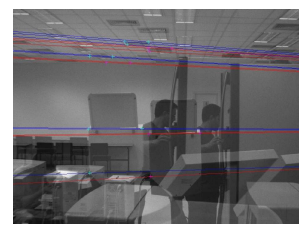

(g)

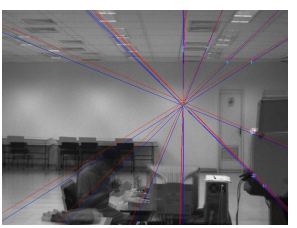

(d)

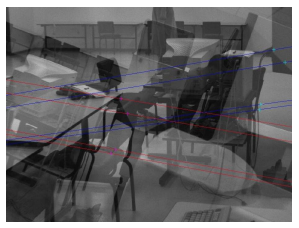

(h)

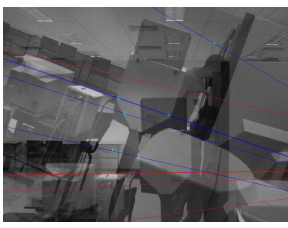

(i)

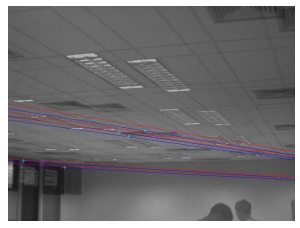

(j)

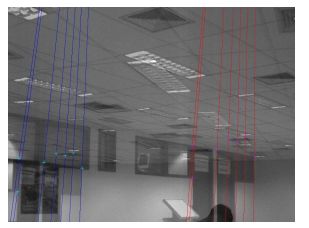

(k)

Figura 9.25: Pontos de teste $3 \mathrm{D}$ da terceira montagem experimental. (a) 00-01. (b) 01-02. (c) 02-03. (d) 03-04. (e) 04-05. (f) 05-06. (g) 06-07. (h) 12-13. (i) 13-14. (j) 15-16. (k) 16-17.

métricas são dadas em metros, e a distância para a reconstrução a partir das matrizes de projeção canônicas é dada em pixel. Em ambos os casos, as matrizes de projeção foram obtidas a partir da matriz fundamental determinada pelo Algoritmo de 8 Pontos Correspondentes Normalizado aplicado sobre o conjunto completo de pares de pontos correspondentes de cada imagem. Desta forma, os dados apresentam o comportamento geral desta medida em relação às duas reconstruções - projetiva (canônica) e métrica.

\subsection{Análise}

Os resultados apresentados nas seções anteriores permitem uma análise dos métodos de estimação de posição por geometria epipolar em três instâncias distintas: determinação da matriz fundamental, cálculo das matrizes de projeção canônicas, e determinação das matrizes de projeção métricas em relação ao sistema de coordenadas global. Em todos estes casos, a quantificação do erro em cada processo permite analisá-los quanto à eficiência e aplicabilidade prática. 
Tabela 9.17: Distância entre os pontos de teste 3D dos blocos K e L na segunda montagem experimental $(\mathrm{m})$.

\begin{tabular}{|c|c|c|c|}
\hline \multirow[t]{2}{*}{ Imagens } & Distância & Reconstrução & Reconstrução \\
\hline & Real (m) & Canônica (pixel) & Euclidiana (m) \\
\hline \multirow[t]{4}{*}{ 01-02 } & 0.4680 & 54.6000 & 0.2534 \\
\hline & 0.4680 & 0.8000 & 0.3193 \\
\hline & 0.4300 & 209.4000 & 0.0382 \\
\hline & 0.3600 & 2393.5000 & 0.0671 \\
\hline \multirow[t]{4}{*}{$02-03$} & 0.4680 & 3313.6000 & 9.1699 \\
\hline & 0.4680 & 116.0000 & 574.5597 \\
\hline & 0.3600 & 421.3000 & 3.5031 \\
\hline & 0.1220 & 208.9000 & 2.2029 \\
\hline \multirow[t]{4}{*}{$04-05$} & 0.4680 & 381.0453 & 0.6132 \\
\hline & 0.4680 & 22.9038 & 0.1823 \\
\hline & 0.4300 & 69.7365 & 0.0168 \\
\hline & 0.3600 & 107.0362 & 0.0154 \\
\hline \multirow[t]{4}{*}{ 01-04 } & 0.4680 & 2967.3000 & 3.3625 \\
\hline & 0.4680 & 38.2000 & 0.3647 \\
\hline & 0.4300 & 702.1000 & 0.0213 \\
\hline & 0.3600 & 468.1000 & 0.0274 \\
\hline \multirow[t]{4}{*}{$01-05$} & 0.4680 & 101.6969 & 2.2474 \\
\hline & 0.4680 & 3.4644 & 0.6492 \\
\hline & 0.4300 & 67.2180 & 1.5707 \\
\hline & 0.3600 & 628.0858 & 0.5961 \\
\hline \multirow[t]{4}{*}{$02-04$} & 0.4680 & 147.1000 & 0.3857 \\
\hline & 0.4680 & 4.4000 & 0.8014 \\
\hline & 0.4300 & 5990.8000 & 0.0435 \\
\hline & 0.3600 & 4546.5000 & 0.0854 \\
\hline \multirow[t]{4}{*}{$02-05$} & 0.4680 & 81.9635 & 0.8811 \\
\hline & 0.4680 & 2.1614 & 0.8041 \\
\hline & 0.4300 & 642.0951 & 0.0363 \\
\hline & 0.3600 & 776.3263 & 0.0688 \\
\hline
\end{tabular}

\subsubsection{Matriz Fundamental}

Nesta etapa, são considerados principalmente os resultados do processamento do conjunto de dados de simulação e do conjunto de dados da primeira montagem experimental. Os resultados do processamento dos conjuntos de dados da segunda e da terceira montagem experimental complementam os primeiros e confirmam as observações feitas com base nos mesmos.

Os resultados apresentados na Tabela 9.1 mostram algumas características dos algoritmos de determinação da matriz fundamental estudados. Por se tratar de um conjunto de dados totalmente gerado por computador os valores das coordenadas dos pares pontos correspondentes selecionados em cada par de imagens é livre de ruído em sua determinação, feita matematicamente com a precisão disponível através do software MATLAB ${ }^{\circledR}$ 6.5. Este ruído nas coordenadas dos pares de pontos correspondentes advém da etapa de sua escolha e marcação, seja este um processo visual por intervenção humana, ou automático a partir de alguma técnica de processamento de imagens. Então, os valores de erro residual observados referem-se ao erro introduzido pelo processo de cálculo de cada método de determinação da matriz fundamental. Além disso, em todos os casos utilizam-se coordenadas de pixel, quantizadas, que só têm significado prático para valores 
Tabela 9.18: Distância entre os pontos de teste 3D dos blocos K e L na terceira montagem experimental $(\mathrm{m})$.

\begin{tabular}{|c|c|c|c|}
\hline Imagens & $\begin{array}{l}\text { Distância } \\
\text { Real (m) }\end{array}$ & $\begin{array}{r}\text { Reconstrução } \\
\text { Canônica (pixel) }\end{array}$ & $\begin{array}{r}\text { Reconstrução } \\
\text { Euclidiana (m) }\end{array}$ \\
\hline \multirow[t]{4}{*}{$00-01$} & 0.4680 & 85.8883 & 0.4659 \\
\hline & 0.4680 & 9.4213 & 0.2210 \\
\hline & 0.4300 & 769.5080 & 0.8760 \\
\hline & 0.3600 & 779.7272 & 1.2936 \\
\hline \multirow[t]{5}{*}{$01-02$} & 0.4680 & 1011.9000 & 0.5022 \\
\hline & 0.4680 & 136.7000 & 0.4786 \\
\hline & 0.4300 & 178.4000 & 0.8112 \\
\hline & 0.3600 & 249.2000 & 1.0315 \\
\hline & 0.6250 & 64.0000 & 0.5531 \\
\hline \multirow[t]{5}{*}{$02-03$} & 0.4680 & 182.2367 & 0.5258 \\
\hline & 0.4680 & 13.9108 & 0.6851 \\
\hline & 0.3600 & 100.9239 & 0.9825 \\
\hline & 0.1220 & 17.4678 & 0.1983 \\
\hline & 0.6250 & 40.7113 & 0.6588 \\
\hline \multirow[t]{5}{*}{$03-04$} & 0.4680 & 133.6062 & 0.5593 \\
\hline & 0.4680 & 12.0718 & 0.1088 \\
\hline & 0.3600 & 284.2164 & 9.8919 \\
\hline & 0.1220 & 42.3797 & 26.3113 \\
\hline & 0.6250 & 49.7225 & 11.8705 \\
\hline \multirow[t]{5}{*}{$04-05$} & 0.4680 & 2545.6000 & 0.5130 \\
\hline & 0.4680 & 401.8000 & 0.5234 \\
\hline & 0.3600 & 385.6000 & 0.8438 \\
\hline & 0.1220 & 152.7000 & 0.1738 \\
\hline & 0.6250 & 95.5000 & 0.6990 \\
\hline \multirow[t]{5}{*}{$05-06$} & 0.4680 & 1647.5000 & 0.4441 \\
\hline & 0.4680 & 20.5000 & 0.7621 \\
\hline & 0.4300 & 1503.9000 & 0.3044 \\
\hline & 0.3600 & 169.6000 & 1.7253 \\
\hline & 0.6250 & 124.8000 & 0.3813 \\
\hline \multirow[t]{5}{*}{$06-07$} & 0.4680 & 4170.6000 & 0.5654 \\
\hline & 0.4680 & 367.2000 & 1.0073 \\
\hline & 0.4300 & 2893.1000 & 0.2022 \\
\hline & 0.3600 & 2901.6000 & 0.9743 \\
\hline & 0.6250 & 331.4000 & 0.4383 \\
\hline \multirow[t]{2}{*}{$12-13$} & 0.3300 & 111.6430 & 0.2793 \\
\hline & 0.1220 & 434.9218 & 0.0474 \\
\hline \multirow[t]{4}{*}{$13-14$} & 0.4000 & 1718.1000 & 0.5055 \\
\hline & 0.1220 & 1348.9000 & 0.1626 \\
\hline & 0 & 0 & 0 \\
\hline & 0 & 0 & 0 \\
\hline \multirow[t]{3}{*}{$15-16$} & 0.4680 & 7524.9000 & 0.4474 \\
\hline & 0.4680 & 6685.2000 & 0.8799 \\
\hline & 2.1700 & 151.3000 & 2.1331 \\
\hline \multirow[t]{4}{*}{$16-17$} & 0.4680 & 153.5202 & 0.4379 \\
\hline & 0.4680 & 157.1714 & 0.6705 \\
\hline & 2.1700 & 244.1998 & 3.3532 \\
\hline & 0.8000 & 462.1884 & 1.0504 \\
\hline
\end{tabular}


inteiros, enquanto que os algoritmos operam no domínio dos números reais.

No caso do Algoritmo de 7 Pontos Correspondentes observa-se erro residual desprezível sobre o conjunto de pontos de entrada, já que o mesmo representa a solução exata sobre este conjunto. Observa-se também que duas das soluções possíveis deste algoritmo apresentam erro residual muito maior que a restante. Esta solução de erro residual menor deve ser considerada a solução correta para a matriz fundamental, e as demais descartadas. Este efeito é mais notável no caso do movimento de translação pura, onde a redução de um grau de liberdade da matriz fundamental faz com que a solução por este método deixe de ser exata. O erro residual observado nesta solução pode ser considerado devido a quantização e aproximação numérica.

No caso do Algoritmo de Busca Exaustiva, escolhe-se a configuração de 7 pares de pontos correspondentes que forneça o menor erro residual dentre todas as combinações possíveis dos pares de pontos correspondentes do conjunto de entrada, aplicando-se o Algoritmo de 7 Pontos Correspondentes a cada uma destas combinações. Este método escolhe automaticamente a solução correta dentre as três possíveis para cada combinação, e também elimina as combinações contendo pontos espúrios - sejam correspondências equivocadas ou pontos muito ruidosos. O erro residual observado com relação ao conjunto de dados de simulação devese à quantização e à possibilidade da escolha aleatória dos pontos colocar muitos deles sobre as mesmas retas epipolares (um efeito que tem maior probabilidade de ocorrer no caso do movimento de translação pura, dada a localização dos pontos correspondentes sobre as arestas do cubo em cada imagem). O desempenho deste método será tanto melhor quanto maior a quantidade de pares de pontos correspondentes do conjunto de entrada, exceto por discrepâncias causadas pelos fatores já mencionados.

Tanto o Algoritmo de 8 Pontos Correspondentes Normalizado como o Algoritmo Linear Iterativo têm um componente a mais de erro inerente ao processo de cálculo por serem baseados em uma solução aproximada por mínimos quadrados. A este somam-se os erros relativos à quantização pixel e os erros numéricos do cálculo, para composição do erro residual apresentado nos resultados. Dada a natureza exata dos pares de pontos correspondentes de entrada, não há diferença entre os resultados destes métodos para o conjunto de dados de simulação. A diferença no desempenho com relação ao erro residual está na quantidade de pares de pontos correspondentes utilizados como entrada destes métodos, que será tanto melhor quanto maior este número. Discrepâncias podem ocorrer pelos mesmos motivo que o caso do Algoritmo de Busca Exaustiva. 
Os resultados do conjunto de dados da primeira montagem experimental, mostrados na Tabela 9.2, reforçam as observações feitas para os resultados do conjunto de dados de simulação. Aqui o erro residual está acrescido do componente de incerteza da matriz fundamental, presente em todos os casos devido ao ruído na determinação dos pares de pontos correspondentes, conforme apresentado no Capítulo 6. A partir destes resultados pode-se determinar que o melhor método em termos de erro residual é o Algoritmo de Busca Exaustiva. Em contrapartida, este é o método de maior complexidade computacional, conforme mostrado no Capítulo 8. O segundo melhor método em termos de erro residual seria o Algoritmo Linear Iterativo, mas este apresenta pouco ganho em relação à sua versão mais simples, o Algoritmo de 8 Pontos Correspondentes Normalizado, principalmente quando a quantidade de pares de pontos correspondentes da entrada é pequena (também observado em (ZHANG, 1996)).

Os resultados observados para os conjuntos de dados da segunda e da terceira montagem experimental mostram um comportamento similar dos algoritmos de determinação da matriz fundamental, com a diferença que o melhor desempenho foi sempre do Algoritmo de 8 Pontos Correspondentes Normalizado. Nestes casos, a resolução das imagens é muito maior que nos casos anteriores, e as cenas presentes nas imagens dos conjuntos de dados têm predominância de pontos mais distantes, onde o foco não é perfeito. Estas características têm influência direta no ruído da determinação dos pares de pontos correspondentes e na presença de correspondências espúrias, cuja influência no erro residual é maior para os métodos baseados no Algoritmo de 7 Pontos Correspondentes. De fato, os resultados da utilização direta do Algoritmo de 7 Pontos Correspondentes foram os piores de todos, e a melhora observada com o Algoritmo de Busca Exaustiva foi menor que a observada com os métodos baseados no Algoritmo de 8 Pontos Correspondentes Normalizado.

\subsubsection{Matrizes de Projeção Canônicas}

Como o cálculo das matrizes de projeção canônicas depende diretamente da matriz fundamental e do epipolo da segunda imagem, como mostrado na Seção 4.4.3, os resultados desta parte do processamento serão tanto melhores quanto maior a precisão da matriz fundamental em relação ao par de imagens. Esta relação pode ser observada indiretamente a partir dos resultados da etapa do cálculo das matrizes de projeção métricas. 


\subsubsection{Matrizes de Projeção Métricas}

Os resultados apresentados nas Tabelas 9.13 e 9.14 mostram o comportamento da determinação das matrizes de projeção métricas em relação aos métodos de determinação da matriz fundamental. Os dados das Tabelas 9.11 e 9.12 mostram a precisão dos métodos de determinação da matriz fundamental em relação aos pontos de referência 3D utilizados para determinação das matrizes de projeção métricas, respectivamente para o conjunto de dados da segunda e da terceira montagem experimental. Estes resultados seguem o mesmo comportamento que os observados na análise dos métodos de determinação da matriz fundamental, e apresentam os melhores valores para o Algoritmo de 8 Pontos Correspondentes Normalizado. No entanto, os resultados da reconstrução métrica dos próprios pontos de referência 3D apresentados nas Tabelas 9.13 e 9.14 têm valores aceitáveis apenas para alguns poucos pares de imagens do conjunto de dados da terceira montagem experimental. Observou-se também que, em todos os casos do conjunto de dados da segunda montagem experimental, a homografia H que transforma a reconstrução projetiva na reconstrução métrica (vide o Teorema da Reconstrução Projetiva, na Seção 4.4.5) obtida a partir dos pontos de referência 3D e das matrizes de projeção canônicas foi sempre mal-condicionada. Também obteve-se H mal-condicionada para os pares de imagens 05-06 e 06-07 do conjunto de dados da terceira montagem experimental. Em todos estes casos foram utilizados exatamente cinco pontos de referência 3D e suas projeções nas imagens consideradas, o mínimo determinado pelo algoritmo de determinação das matrizes de projeção métricas. Outra observação sobre estes casos foi que o erro elevado deveu-se a discrepâncias na posição de um único ponto, concentradas principalmente na coordenada $\mathrm{z}$, perpendicular ao plano de imagem e correspondente à profundidade do ponto. Nas situações onde foram determinados mais pontos de referência 3D, a matriz H não apresentou problemas de condicionamento, mas em alguns casos os resultados da reconstrução métrica também não foram satisfatórios (pares de imagens 02-03, 03-04, 13-14 e 16-17 do conjunto de dados da terceira montagem experimental). Nestes casos o erro também ficou concentrado principalmente em um ponto, e em alguns casos houve um segundo ponto com erro elevado, mas sempre bem menor que o principal. E também aqui a maior discrepância deveu-se à profundidade, representando cerca de $10 \%$ do valor da coordenada $\mathrm{Z}$ dos pontos discrepantes, no pior caso.

Observando as imagens das Figuras 9.22 e 9.23, pode-se inferir que mesmo quando a matriz fundamental utilizada para determinar a reconstrução métrica apresenta um pequeno erro residual com relação aos pontos de referência 3D, 
pequenos ruídos nas coordenadas dos pontos correspondentes projetados destes pontos de referência representam erros consideráveis na reconstrução métrica, e que o conjunto mínimo de cinco pontos de referência 3D é na prática insuficiente, devido a estes mesmos ruídos e à grande possibilidade de correspondências espúrias.

Os resultados apresentados nas Tabelas 9.15 e 9.16 mostram a acuracidade da reconstrução métrica com respeito à estimativa de posição de elementos da cena em cada par de imagens. Considerando apenas os casos em que o resultado da reconstrução métrica dos pontos de referência 3D apresentou erro aceitável, especificamente dos pares de imagens 00-01, 01-02, 04-05, 12-13 e 15-16, observa-se que os pontos de teste 3D escolhidos próximos aos pontos de referência apresentam todos pequeno erro na reconstrução métrica. Estes pontos de teste 3D definem os mesmos planos definidos pelos pontos de referência 3D de que estão próximos. Mesmo nestes casos, o principal componente do erro deve-se a discrepâncias na coordenada Z. Os pontos de teste 3D que apresentam erro elevado na reconstrução métrica estão distantes em profundidade dos pontos de referência 3D, e o erro deve-se a discrepâncias em todas coordenadas. Isto explica-se devido à característica da reconstrução ser possível a menos de uma transformação de similaridade para o caso de câmeras calibradas, ou a menos de uma transformação projetiva para o caso de câmeras não calibradas. Então, como as matrizes de projeção métricas são determinadas a menos de um fator de escala, os ruídos na determinação dos pontos correspondentes - tanto na determinação da matriz fundamental como na determinação da homografia $\mathrm{H}$ da reconstrução métrica e os erros devido a correspondências espúrias presentes nos conjuntos de dados analisados produzem erros elevados nas coordenadas Euclidianas dos pontos reconstruídos, mesmo quando as discrepâncias originais forem pequenas em termos das coordenadas de pixel dos pontos das imagens.

Os resultados das Tabelas 9.17 e 9.18 apresentam o comportamento de medidas de distância entre pontos mediante a reconstrução projetiva (canônica) e a reconstrução métrica. Observa-se que estes resultados seguem o mesmo comportamento descrito anteriormente para a reconstrução métrica dos pontos de teste 3D, e que distâncias medidas em profundidade apresentam erro elevado. Distâncias medidas em planos paralelos ao plano da imagem e em regiões próximas às determinadas pelos pontos de referência 3D têm aproximações razoáveis pela reconstrução métrica.

A partir destas análises a reconstrução métrica e conseqüente estimação de posição pode ser levada com precisão aceitável dentro do campo das imagens de 


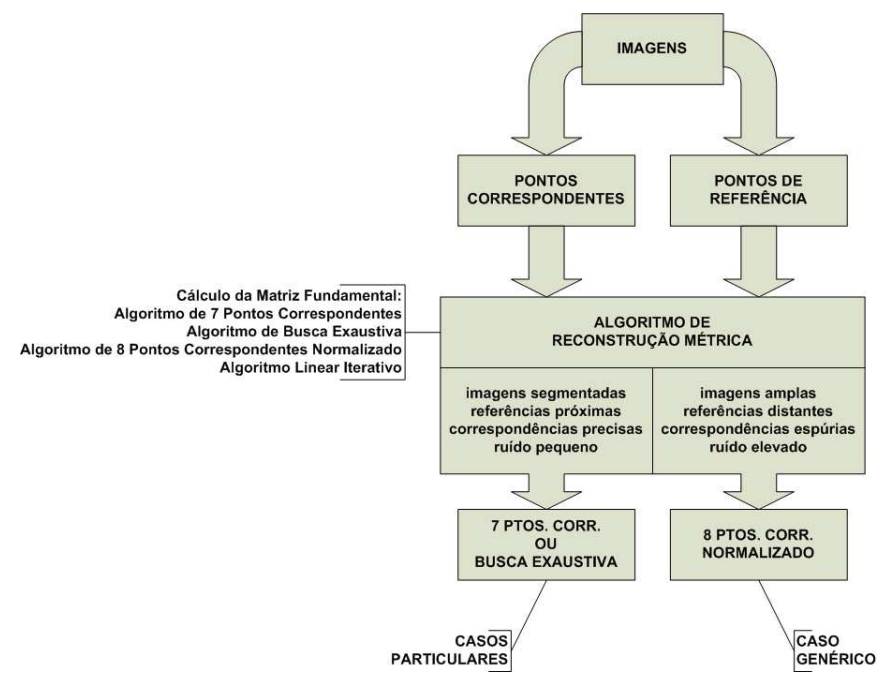

Figura 9.26: Resumo da análise dos resultados.

uma cena, considerando regiões e objetos específicos desta cena, precisão esta tanto melhor quanto mais acurados os pontos correspondentes relativos aos pontos de referência 3D utilizados na determinação das matrizes de projeção métricas, e também dependendo da eficiência do método de determinação da matriz fundamental. A Figura 9.26 mostra o resumo da análise dos resultados, apresentando a melhor escolha de algoritmo de cálculo da matriz fundamental no processo de reconstrução métrica, de acordo com as condições de acuidade dos pontos correspondentes e disponibilidade de referências. 


\section{Conclusão}

De forma geral, o ponto que diferencia os métodos de estimação de posição por geometria epipolar entre imagens apresentados neste trabalho é o algoritmo de determinação da matriz fundamental. As demais etapas do processo de reconstrução métrica - determinação da reconstrução projetiva, triangulação e retificação para reconstrução métrica - não apresentam variantes. Apesar de não representarem variações do algoritmo de estimação de posição, estas etapas têm grande influência nos resultados, dependendo das condições em que são executadas, e também devem ser cuidadosamente analisados.

A análise dos resultados práticos combinada com o estudo de complexidade computacional apresentado no Capítulo 8 sugerem que a melhor escolha de método de determinação da matriz fundamental é o Algoritmo de 8 Pontos Correspondentes Normalizado. O Algoritmo de 8 Pontos Correspondentes Normalizado já foi apontado como um dos métodos de determinação da matriz fundamental de melhor desempenho em (HARTLEY; ZISSERMAN, 2000), (XU; ZHANG, 1996) e (ZHANG, 1996), mas sem considerações detalhadas quanto ao erro e à complexidade computacional, bem como as condições de sua aplicação, detalhadas na Seção 9.3 e sumarizadas na Figura 9.26.

Apesar de observados bons resultados quanto ao algoritmo de busca exaustiva, o aumento significativo da complexidade computacional pode tornar esta alternativa inviável para grandes conjuntos de dados. Conforme observado em outras pesquisas (ZHANG, 1996), o Algoritmo Linear Iterativo, extensão do Algoritmo de 8 Pontos Correspondentes Normalizado, não apresenta melhora significativa em relação à sua versão mais simples, com um custo em processamento bastante superior. Naturalmente, grandes conjuntos de dados fornecem os melhores resultados em termos de erro residual, mas a obtenção de pontos correspondentes entre imagens com precisão é um tarefa complexa, que seria interessante reduzir ao máximo.

Segundo estas considerações, podem ser levantados alguns panoramas para utilização de cada método. Se a precisão do sistema de obtenção de pontos correspondentes entre as imagens for elevada, o Algoritmo de 7 Pontos Correspon- 
dentes parece ser a melhor opção, pois reduz consideravelmente a complexidade computacional. Para uns poucos pontos a mais, caso seja necessário eliminar os efeitos de alguma correspondência espúria, é possível estender este método para o Algoritmo de Busca Exaustiva, sem que o custo em processamento se torne proibitivo. Quando o conjunto de pontos correspondentes entre imagens estiver sujeito a ruídos e dados espúrios, mas estiverem disponíveis em grande número, o método de cálculo da matriz fundamental que melhor se adapta é o Algoritmo de 8 Pontos Correspondentes Normalizado. Este método fornece a solução que melhor de adapta aos pontos correspondentes como um todo, compensando a falta de qualidade de algumas medições. Outra consideração interessante observada nos resultados práticos é que se for possível determinar regiões de interesse na imagem, é possível aplicar o algoritmo de determinação da matriz fundamental em pontos correspondentes de cada região separadamente, com resultados setorizados superiores aos resultados globais. Neste sentido, pode-se aplicar o Algoritmo de 7 Pontos Correspondentes em regiões específicas da imagem, e nas etapas seguintes obter a retificação métrica válida nesta região. No entanto, a localização de regiões de interesse em uma imagem apresenta o mesmo problema de complexidade que a determinação das correspondências. Estes pontos representam na verdade um outro ramo da visão computacional, o reconhecimento de objetos ou padrões.

Os resultados experimentais mostram também o efeito das incertezas inerentes ao processo de determinação da matriz fundamental. Utilizando dados de imagens geradas por computador, onde as correspondências são exatas e não há erros de medição, observa-se em todos os casos a presença de erro residual no cálculo da matriz fundamental. Este erro é devido às medidas nas imagens serem quantizadas enquanto a determinação da matriz fundamental é realizada no domínio real. Para efeitos práticos, a precisão numérica dos algoritmos em MATLAB ${ }^{\circledR}$ pode ser considerada infinita. Outro fator que influencia a eficiência dos algoritmos de determinação da matriz fundamental é a escolha das correspondências entre as imagens, presente em todos resultados práticos mas visível principalmente naqueles da segunda montagem experimental. Devido à quantização e ao ruído de medição, a posição de alguns pares de pontos correspondentes pode levar às mesmas retas epipolares em cada imagem, gerando sistemas de equações mal-condicionados e uma estimativa ruim da matriz fundamental. Os resultados obtidos das montagens experimentais com câmeras reais mostram o erro residual composto pelo mesmo erro de quantização e pela incerteza da matriz fundamental, descrita no Capítulo 6. Observado isoladamente, este erro parece desprezível, 
mas ele indica quão bom será o desempenho do processo de estimação de posição como um todo, e tem influência direta nos resultados das próximas etapas.

Os resultados da reconstrução projetiva, definida pelas matrizes de projeção canônica, dependem unicamente do desempenho obtido na determinação da matriz fundamental. Erros introduzidos por esta etapa são apenas referentes a precisão numérica dos cálculos matriciais envolvidos.

Em termos dos resultados práticos, as etapas de triangulação e retificação para reconstrução métrica devem ser consideradas em conjunto, uma vez que dependem do conjunto de pontos de referência do espaço tridimensional escolhidos para determinar a homografia de retificação. As mesmas considerações feitas para a incerteza da matriz fundamental no Capítulo 6 também se aplicam à determinação desta homografia, uma vez que o algoritmo utilizado também utiliza SVD para obter a solução. Sendo assim, a qualidade na determinação dos pontos correspondentes relativos aos pontos de referência é crucial para o desempenho desta etapa. Independente deste fator, se a matriz fundamental obtida na primeira etapa não representar uma boa estimativa da matriz fundamental real, todas as etapas posteriores serão prejudicadas.

Uma observação interessante dos resultados práticos é que o desempenho da reconstrução métrica pode ser melhorado quando se considera apenas uma região específica da imagem. Pontos reconstruídos dentro de uma área próxima àquela dos pontos de referência apresentam um erro aceitável, enquanto pontos reconstruídos em regiões afastadas dos pontos de referência apresentam erro elevado. Outra observação é que a profundidade - distância do ponto ao centro de câmera na direção do eixo principal - é a dimensão que apresenta maior erro na estimativa. Além do erro inerente ao processo, este efeito pode ser explicado pela escolha de pontos de referência distantes do centro de câmera, para os quais a determinação dos pontos correspondentes nas imagens é prejudicada por questões de foco e quantização.

Sumarizando a análise dos resultados práticos, pode-se concluir que um sensor de visão de propósito geral pode ser construído utilizando estimação de posição através do Algoritmo de 8 Pontos Correspondentes Normalizado. Este sistema não requer hardware computacional muito sofisticado e um pré-processamento moderado para obter um bom volume de pontos correspondentes cuja precisão deve ser boa, mas onde eventuais discrepâncias podem ser ajustadas. De maneira geral, a aplicação deste sensor depende de um ambiente com referências de fácil identificação, distribuídas conforme regiões de interesse para a estimação de 
posição.

Considerando os próximos passos para utilização efetiva da estimação de posição por geometria epipolar em um sensor de visão de propósito geral, a análise dos resultados deixa evidente que o desempenho deste sensor depende muito da inteligência na determinação de pontos correspondentes ou regiões de interesse nas imagens. Neste trabalho, para efeito de cálculo e quantificação de erro, a obtenção dos pontos correspondentes foi considerada uma tarefa externa ao processo de estimação de posição - são os dados de entrada fornecidos ao processo. E o processo de escolha dos pontos correspondentes e das referências foi feita através da inteligência humana. Traduzir esta inteligência para um algoritmo capaz de ser implementado em um computador da atualidade é uma tarefa de grande complexidade, objeto de pesquisas do estado-da-arte da ciência da computação. Neste sentido, uma área promissora é a de autômatos adaptativos - que introduz um formalismo com regras dinamicamente variáveis, tornando um algoritmo capaz de aprendizado contínuo durante sua operação, e criando condições de implementar a inteligência necessária para realizar tarefas de grande complexidade. Assim, a utilização de técnicas de autômatos adaptativos em visão computacional pode representar o passo seguinte para obtenção de um sensor de visão completo e de propósito geral, e deve ser objeto de estudos futuros. 


\section{Referências}

ACAR M.; PARKIN, R. M. Engineering education for mechatronics. IEEE Transactions on Industrial Electronics, IEEE Industrial Electronics Society, v. 43, n. 1, p. 106-112, February 1996.

ANDERSON, T. W. An Introduction to Multivariate Statistical Analysis. New York: Wiley \& Sons, Inc., 1958.

ARMANGUÉ, X.; SALVI, J. Overall view regarding fundamental matrix estimation. Image and Vision Computing, v. 21, p. 205-220, February 2003. Disponível em: <http://vicorob.udg.es/fitxers/articles/doc/200.pdf>.

BLACK, M. J.; SAPIRO, G.; MARIMONT, D.; HEEGER, D. Robust anisotropic diffusion. IEEE Trans. on Image Processing, v. 7, n. 3, p. 421-432, 1998.

CACCIA, M. Vision-Based SLAM for ROVs: Preliminary Experimental Results. September 2006. IFAC MCMC'2006: 7th Conference on Manoeuvring and Control of Marine Craft.

CHEONG, L.-F.; PEH, C.-H. Depth distortion under calibration uncertainty. Comput. Vis. Image Underst., Elsevier Science Inc., New York, NY, USA, v. 93, n. 3, p. 221-244, 2004. ISSN 1077-3142.

CRIMINISI, A. Accurate Visual Metrology from Single and Multiple Uncalibrated Images. Tese (Doutorado) - University of Oxford, Dept. Engineering Science, December 1999. D.Phil. thesis. Disponível em: $<$ http://www.robots.ox.ac.uk/ ${ }^{\sim}$ vgg $>$.

CRIMINISI, A.; REID, I. D.; ZISSERMAN, A. Single view metrology. In: Proceedings of the 7th International Conference on Computer Vision. [S.l.: s.n.], 1999. p. 434-441.

DERICHE, R.; ZHANG, Z.; LUONG, Q.-T.; FAUGERAS, O. Robust recovery of the epipolar geometry for an uncalibrated stereo rig. In: ECCV'94: Proceedings of the third European conference on Computer vision (vol. 1). Secaucus, NJ, USA: Springer-Verlag New York, Inc., 1994. p. 567-576. ISBN 3-540-57956-7.

FAUGERAS, O. Three-dimensional computer vision: a geometric viewpoint. Cambridge, MA, USA: MIT Press, 1993. ISBN 0-262-06158-9.

GIBSON, J. J. The Perception of the Visual World. Boston, MA, USA: Houghton Mifflin, 1955.

GONZALEZ, R. C.; WOODS, R. E. Digital Image Processing. Boston, MA, USA: Addison-Wesley Longman Publishing Co., Inc., 1992. ISBN 0201508036. 
GRACIAS, N.; SANTOS-VICTOR, J. Underwater video mosaics as visual navigation maps. Comput. Vis. Image Underst., Elsevier Science Inc., New York, NY, USA, v. 79, n. 1, p. 66-91, 2000. ISSN 1077-3142.

HARTLEY, R. I. Projective reconstruction and invariants from multiple images. $P A M I$, v. 16, n. 10, p. 1036-1041, October 1994.

HARTLEY, R. I. In defense of the eight-point algorithm. IEEE Trans. Pattern Anal. Mach. Intell., IEEE Computer Society, Washington, DC, USA, v. 19, n. 6, p. 580-593, 1997. ISSN 0162-8828.

HARTLEY, R. I. Lines and points in three views and the trifocal tensor. Int. J. Comput. Vision, Kluwer Academic Publishers, Hingham, MA, USA, v. 22, n. 2, p. 125-140, 1997. ISSN 0920-5691.

HARTLEY, R. I.; ZISSERMAN, A. Multiple View Geometry in Computer Vision. [S.1.]: Cambridge University Press, ISBN: 0521623049, 2000.

HORGAN, J.; TOAL, D. Vision Systems in the Control of Autonomous Underwater Vehicles. September 2006. IFAC MCMC'2006: 7th Conference on Manoeuvring and Control of Marine Craft.

HORN, R. A.; JOHNSON, C. R. Matrix Analysis. [S.l.]: Cambridge University Press, 1990. ISBN 0521386322.

LEWIS, H. R.; PAPADIMITRIOU, C. H. Elements of the Theory of Computation. Upper Saddle River, NJ, USA: Prentice Hall PTR, 1997. ISBN 0132624788.

LONGUETT-HIGGINS, H. C. A computer algorithm for reconstruction of a scene from two projections. Nature, p. 293:133-135, September 1981.

LUONG, Q.-T.; DERICHE, R.; FAUGERAS, O.; PAPADOPOULO, T. On Determining the Fundamental Matrix: Analysis of Different Methods and Experimental Results. [S.l.], 1993. Disponível em: <http://www.inria.fr/rapports/sophia/RR-1894.html>.

LUONG, Q.-T.; VIÉVILLE, T. Canonical representations for the geometries of multiple projective views. Comput. Vis. Image Underst., Elsevier Science Inc., New York, NY, USA, v. 64, n. 2, p. 193-229, September 1996. ISSN 1077-3142.

MARANI, G.; YUH, J. Vision-Based Control in Autonomous Manipulation for Intervention Missions AUV. September 2006. IFAC MCMC'2006: 7th Conference on Manoeuvring and Control of Marine Craft.

MARR, D. Vision: A Computational Investigation into the Human Representation and Processing of Visual Information. New York, NY, USA: Henry Holt and Co., Inc., 1982. ISBN 0716715678.

MARTINS, A.; ALMEIDA, J. M.; SILVA, E. P.; PEREIRA, F. L. Vision-Based Autonomous Surface Vehicle Docking Manoeuvre. September 2006. IFAC MCMC'2006: 7th Conference on Manoeuvring and Control of Marine Craft.

MAYBANK, S. Theory of Reconstruction from Image Motion. Berlin, Germany: Springer-Verlag, 1993. ISBN 3-540-55537-4. 
MOON, I. H.; MIURA, J.; YANAGI, Y.; SHIRAI, Y. Planning of Vision-Based Navigation for a Mobile Robot under Uncertainty. September 1997. Proc. of IEEE/RSJ Int. Conf. on Intelligent Robots and Systems, p. 1202-1207.

OKAMOTO JR, J.; GRASSI JR, V.; CUNHA, F. M.; MOSCATO, L. A. Target location task with omnidirectional vision system. In: IASTED 2002 Visualization, Imaging, and Image Processing Conference. [S.l.: s.n.], 2002. p. 90-95.

PERONA, P.; MALIK, J. Scale-space and edge detection using anisotropic diffusion. IEEE Trans. Pattern Anal. Mach. Intell., IEEE Computer Society, Washington, DC, USA, v. 12, n. 7, p. 629-639, 1990. ISSN 0162-8828.

PLAKAS, K.; TRUCCO, E. Developing a Real-Time, Robust, Video Tracker. September 2000. MTS/IEEE Oceans 2000, p. 1345-1352.

SEMPLE, J. G.; KNEEBONE, G. T. Algebraic Projective Geometry. Oxford, England: Oxford University Press, 1952. ISBN 0-19-8503636.

SHIMADA, M.; TSUZUKI, M. S. G. Implementação de operações booleanas usando aritmética intervalar em um modelador de sólidos B-REP. In: Anais do XVI COBEM. [S.1.]: ABCM, 2001.

SONG, F.; AN, P. E.; FOLLECO, A. Modeling and simulation of autonomous underwater vehicles: design and implementation. IEEE Journal of Oceanic Engineering, IEEE Oceanic Engineering Society, v. 28, n. 2, p. 283- 296, 2003. ISSN 0364-9059.

TORR, P. H. S.; ZISSERMAN, A. Robust parameterization and computation of the trifocal tensor. Image and Vision Computing, v. 15, p. 591-605, 1997. Disponível em: <http://www.robots.ox.ac.uk/ ${ }^{\sim} \operatorname{vgg}>$.

TSAI, R. An efficient and accurate camera calibration technique for 3d machine vision. IEEE CVPR, p. 364-374, 1986.

TSUZUKI, M. S. G. Solucionando sistemas polinomiais não lineares pelo método do poliedro projetado implementado em aritmética intervalar. 2000. EPUSP. Tese (Livre Docência).

TSUZUKI, M. S. G.; ALMEIDA, A. G.; NISHIMOTO, K. Extracting automatically hydrodynamic information from a $3 \mathrm{~d}$ cad to aid the project of rovs. In: IASTED 6th. Robotics and Manufacturing International Conference. [S.l.: s.n.], 1998. p. 264-267.

ULLMAN, S. The Interpretation of Visual Motion. Cambridge, MA, USA: MIT Press, 1979.

XU, G.; ZHANG, Z. Epipolar Geometry in Stereo, Motion, and Object Recognition: A Unified Approach. Norwell, MA, USA: Kluwer Academic Publishers, 1996. ISBN 0792341996.

ZHANG, Z. Determining the Epipolar Geometry and its Uncertainty: A Review. [S.l.], 1996. Disponível em: <http://www.inria.fr/rapports/sophia/RR2927.html>. 
ZWAAN, S. van der; BERNARDINO, A.; SANTOS-VICTOR, J. Visual station keeping for floating robots in unstructured environments. Robotics and Autonomous Systems, v. 39, n. 3-4, p. 145-155, 2002. 


\section{Apêndice A - Conceitos Adicionais}

\section{A.1 Rotação Conjugada}

Uma matriz de rotação $R$ tem autovalores $\left\{1, \mathrm{e}^{\mathrm{i} \theta}, \mathrm{e}^{-\mathrm{i} \theta}\right\}$, com correspondentes autovetores $\{\mathbf{a}, \mathbf{I}, \mathbf{J}\}$, onde a é o vetor do eixo de rotação tal que $R \mathbf{a}=\mathbf{a}, \theta$ é o ângulo de rotação em torno deste eixo, e I e $\mathbf{J}$ (complexos conjugados) são os pontos circulares do plano ortogonal a a. Dada uma transformação projetiva entre dois planos da forma $\mathrm{H}=\mathrm{TRT}^{-1}$, onde $\mathrm{T}$ é uma transformação projetiva qualquer, então H é uma rotação conjugada. Uma rotação conjugada preserva os autovalores, de forma que os autovalores da transformação projetiva H também são $\left\{1, \mathrm{e}^{\mathrm{i} \theta}, \mathrm{e}^{-\mathrm{i} \theta}\right\}$, a menos de um fator de escala em comum.

\section{A.2 Produto Vetorial}

\section{A.2.1 Representação Matricial}

Pode-se representar o produto vetorial de dois vetores pelo produto de uma matriz anti-simétrica $3 \times 3$ derivada de um dos vetores pelo outro vetor. Através deste artifício pode-se realizar operações matriciais com produtos vetoriais.

Dado um vetor de três dimensões $\mathbf{x}=\left(x_{1}, x_{2}, x_{3}\right)^{\mathrm{T}}$, define-se uma matriz anti-simétrica $3 \times 3$ correspondente a $\mathbf{x}$ por:

$$
[\mathbf{x}]_{\times}=\left[\begin{array}{ccc}
0 & -x_{3} & x_{2} \\
x_{3} & 0 & -x_{1} \\
-x_{2} & x_{1} & 0
\end{array}\right]
$$

Nota-se que qualquer matriz anti-simétrica $3 \times 3$ pode ser escrita na forma $[\mathbf{x}]_{\times}$para algum vetor $\mathbf{x}$. A matriz $[\mathbf{x}]_{\times}$é singular e seu espaço nulo é $\mathbf{x}$.

$\mathrm{O}$ produto vetorial $\mathbf{a} \times \mathbf{b}($ ou $\mathbf{a} \wedge \mathbf{b})$ de dois vetores tridimensionais $\mathbf{a}=$ $\left(a_{1}, a_{2}, a_{3}\right)^{\mathrm{T}}$ e $\mathbf{b}=\left(b_{1}, b_{2}, b_{3}\right)^{\mathrm{T}}$, é o vetor $\left(a_{2} b_{3}-a_{3} b_{2}, a_{3} b_{1}-a_{1} b_{3}, a_{1} b_{2}-a_{2} b_{1}\right)^{\mathrm{T}}$. 
Desta forma, $\mathbf{a} \times \mathbf{b}$ pode ser escrito como:

$$
\mathbf{a} \times \mathbf{b}=[\mathbf{a}]_{\times} \mathbf{b}=\left(\mathbf{a}^{\mathrm{T}}[\mathbf{b}]_{\times}\right)^{\mathrm{T}}
$$

\section{A.2.2 Operação Matricial}

Dado um vetor $3 \mathrm{D}$ qualquer t e uma matriz $3 \times 3$ não-singular M, então:

$$
[\mathbf{t}]_{\times} \mathrm{M}=\mathrm{M}^{-\mathrm{T}}\left[\mathrm{M}^{-1} \mathbf{t}\right]_{\times}
$$

Demonstra-se que para qualquer matriz $3 \times 3$ e quaisquer vetores $3 \mathrm{D}$ x e $\mathbf{y}$, vale a relação:

$$
(\mathrm{Mx}) \times(\mathrm{My})=\mathrm{M}^{*}(\mathbf{x} \times \mathbf{y}) \quad \text { ou } \quad[\mathrm{Mx}]_{\times} \mathrm{M}=\mathrm{M}^{*}[\mathbf{x}]_{\times},
$$

onde $\mathrm{M}^{*}$ é a matriz de cofatores de $\mathrm{M}$. Define-se a matriz de cofatores de $\mathrm{M}$ por $\mathrm{M}^{*}=\left[m_{i j}^{*}\right]$, onde $m_{i j}^{*}=(-1)^{i+j} \operatorname{det}\left(\hat{\mathrm{M}}_{i j}\right)$, com $\hat{\mathrm{M}}_{i j}$ representando a submatriz de $\mathrm{M}$ obtida eliminando-se a linha $i$ e a coluna $j$.

Se M é inversível, então $M^{*}=\operatorname{det}(\mathrm{M}) \mathrm{M}^{-\mathrm{T}}$. Neste caso, as colunas de $\mathrm{M}$ constituem uma base do espaço tridimensional, e qualquer vetor 3D pode ser escrito como uma combinação linear destas colunas. Assim, tomando $\mathbf{t}=\mathbf{M x}$ e desprezando o fator de escala $\operatorname{det}(\mathbf{M})$, obtém-se $[\mathbf{t}]_{\times} M=M^{-T}\left[M^{-1} \mathbf{t}\right]_{\times}$.

\section{A.3 Rotação e Translação Tridimensional}

Denota-se $R_{\mathbf{a}}(\theta)$ a rotação $3 \mathrm{D}$ de um ângulo $\theta$ em torno de um eixo qualquer a. A matriz $R_{\mathbf{a}}(\theta)$ pode ser determinada sistematicamente através da seguinte série de transformações: uma translação $T_{\mathbf{a}}$ do espaço, de forma que o eixo de rotação passe pela origem do sistema de coordenadas; uma rotação do espaço $\mathrm{R}_{\mathrm{XZ}}$ em torno do eixo $\mathrm{Z}$, de forma que o eixo de rotação fique sobre o plano XZ; uma rotação do espaço $R_{X Z 2 Z}$ em torno do eixo $Y$, de forma que o eixo de rotação coincida com o eixo $\mathrm{Z}$; uma rotação $\mathrm{R}_{Z}(\theta)$ do ângulo $\theta$ em torno do eixo $\mathrm{Z}$; as rotações inversas $R_{X Z 2 Z}^{-1}$, seguida de $R_{X Z}^{-1}$, e a translação inversa $T_{\mathbf{a}}^{-1}$, de forma a retornar o espaço à sua posição original em relação ao eixo de rotação. Assim, tem-se: 


$$
\mathrm{R}_{\mathbf{a}}(\theta)=\mathrm{T}_{\mathbf{a}}^{-1} \mathrm{R}_{\mathrm{XZ}}^{-1} \mathrm{R}_{\mathrm{XZ2Z}}^{-1} \mathrm{R}_{\mathrm{Z}}(\theta) \mathrm{R}_{\mathrm{XZ2Z}} \mathrm{R}_{\mathrm{XZ}} \mathrm{T}_{\mathbf{a}} .
$$

De forma a analisar a composição de uma rotação com uma translação, observa-se dada uma direção de translação t do espaço tridimensional, tem-se:

$$
\begin{aligned}
{[t]_{\times} R_{\mathbf{a}}(\theta) } & =[t]_{\times}\left(T_{\mathbf{a}}^{-1} R_{X Z}^{-1} R_{X Z 2 Z}^{-1} R_{Z}(\theta) R_{X Z 2 Z} R_{X Z} T_{\mathbf{a}}\right) \\
& =R_{X Z 2 Z}^{\mathrm{T}} R_{X Z}^{\mathrm{T}} T_{a}^{\mathrm{T}}\left[R_{X Z 2 Z} R_{X Z} T_{\mathbf{a}} t\right]_{\times} R_{Z}(\theta) R_{X Z 2 Z} R_{X Z} T_{\mathbf{a}} .
\end{aligned}
$$

O produto $R_{X Z 2 Z} R_{X Z} T_{a} t$ equivale a translação e rotação do espaço de forma que $\mathbf{t}$ coincida com o eixo $\mathrm{Z}$. O produto $\mathrm{R}_{\mathrm{Z}}(\theta) \mathrm{R}_{\mathrm{XZ2Z}} \mathrm{R}_{\mathrm{XZ}} \mathrm{T}_{\mathbf{a}}$, corresponde à rotação de um ângulo $\theta$ em torno do eixo de rotação coincidente com o eixo Z. Assim, o produto $[\mathbf{t}]_{\times} \mathbf{R}_{\mathbf{a}}(\theta)$ equivale ao produto vetorial $\mathbf{t} \times \mathbf{a}$, entre a direção de translação t e o eixo de rotação a. Se o eixo de rotação a é ortogonal a um plano $\pi$, então $\mathbf{t} \times \mathbf{a}$ está sobre este plano e tem dois graus de liberdade. Considerando a direção de translação $\mathbf{t}$ ortogonal ao eixo de rotação $\mathbf{a}, \mathbf{t} \times \mathbf{a}$ passa a ter apenas um grau de liberdade.

\section{A.4 Decomposição em Valores Singulares}

Na Álgebra Linear, a decomposição em valores singulares - SVD (Singular Value Decomposition) - é uma forma importante de fatorização de matrizes retangulares reais ou complexas, com variadas aplicações em estatística e processamento de sinais.

Dada uma matriz M $m \times n$, então a decomposição em valores singulares de $\mathrm{M}$ é a fatoração:

$$
\mathrm{M}=\mathrm{U \Sigma} \mathrm{V}^{*}
$$

onde U é uma matriz unitária (ortonormal) $m \times m, \Sigma$ é a matriz diagonal $m \times n$ dos valores singulares de $\mathrm{M}$, e $\mathrm{V}^{*}$ denota a matriz transposta conjugada de $\mathrm{V}$, a matriz $n \times n$ dos vetores singulares (à direita) de M.

Um número real positivo $\sigma$ é um valor singular de M se e somente se existirem vetores normalizados $\mathbf{u}$ e $\mathbf{v}$, respectivamente com $m$ e $n$ elementos, tais que:

$$
\mathbf{M} \mathbf{v}=\sigma \mathbf{u} \mathbf{e ~ M}^{*} \mathbf{u}=\sigma \mathbf{v}
$$


Os vetores $\mathbf{u}$ e $\mathbf{v}$ são chamados respectivamente de vetores singulares à esquerda e à direita de $\sigma$. Os elementos da diagonal de $\Sigma$ são os valores singulares de M, normalmente ordenados de forma decrescente. As colunas das matrizes U e V são respectivamente os vetores singulares à esquerda e à direita do valor singular correspondente em $\Sigma$. Assim, uma matriz $\mathrm{M} m \times n$ tem no máximo $p=\min (m, n)$ valores singulares distintos.

\section{A.5 Norma de Frobenius}

Conforme (HORN; JOHNSON, 1990), a norma de Frobenius de uma matriz M $m \times n$ é dada por:

$$
\|\mathrm{M}\|_{\mathrm{F}}^{2}=\sum_{i=1}^{m} \sum_{j=1}^{m}\left|m_{i j}\right|^{2}=\operatorname{trc}\left(\mathrm{MM}^{*}\right)=\sum_{i, j}^{\min (m, n)} \sigma_{i}^{2} .
$$

onde a função $\operatorname{trc}(\cdot)$ representa o traço da matriz quadrada (soma dos elementos da diagonal principal), $\mathrm{M}^{*}$ é a matriz hermitiana de $\mathrm{M}$ (transposta conjugada), e $\sigma_{i}, i=1, \ldots, \min (m, n)$, são os valores singulares de M.

\section{A.6 Normalização}

O condicionamento de uma matriz é a razão entre o primeiro e o penúltimo valor singular (em ordem decrescente). No caso do cálculo da matriz fundamental através do Algoritmo de 8 Pontos Correspondentes, descrito na Seção 5.2, a presença de ruído nas coordenadas dos pontos correspondentes causa divergência entre a solução do sistema $\mathbf{A f}=\mathbf{0}$ e o valor correto de $\mathbf{f}$, e esta divergência será tanto maior quanto maior for o condicionamento da matriz A. Conforme demonstrado por Hartley (HARTLEY, 1997a), o condicionamento da matriz pode ser melhorado tanto pelo deslocamento do centróide dos pontos correspondentes em cada imagem para a origem do sistema de coordenadas de imagem (suposta no canto superior esquerdo), como pelo escalonamento dos eixos de coordenadas de forma que as coordenadas homogêneas médias sejam unitárias.

A solução do sistema de equações Af $=\mathbf{0}$ através da SVD da matriz A equivale a determinar:

$$
\min _{\mathrm{F}} \sum_{i} \mathbf{x}^{\prime \mathrm{T}} \mathrm{F} \mathbf{x}
$$


A distância Euclidiana entre o ponto $\mathbf{x}^{\prime}$ da segunda imagem e a reta epipolar $\mathbf{l}^{\prime}=\mathbf{F} \mathbf{x}=\left(l_{1}^{\prime}, l_{2}^{\prime}, l_{3}^{\prime}\right)^{\mathrm{T}}$ do ponto correspondente da primeira imagem é dada por:

$$
\mathrm{d}\left(\mathbf{x}^{\prime}, \mathbf{l}^{\prime}\right)=\left|\mathbf{x}^{\prime \mathrm{T}} \mathbf{l}^{\prime}\right| / \sqrt{l_{1}^{2}+l_{2}^{\prime 2}} .
$$

Esta expressão pode ser considerada sempre válida, uma vez que o fator de escalonamento $k=\sqrt{l_{1}^{\prime 2}+l_{2}^{\prime 2}}$ assume o valor nulo somente em casos degenerados, como quando a reta epipolar está no infinito. A solução do sistema de equações Af $=\mathbf{0}$ será então:

$$
\min _{\mathrm{F}} \sum_{i} k_{i}^{2} \mathrm{~d}\left(\mathbf{x}^{\prime}, \mathbf{l}^{\prime}\right)^{2}
$$

Conforme Luong, Deriche, Faugeras e Papadopoulo (LUONG et al., 1993), a solução minimiza tanto $k$ como $\mathrm{d}\left(\mathbf{x}^{\prime}, \mathbf{l}^{\prime}\right)^{2}$. A minimização de $k$ representa privilegiar as soluções cujos epipolos estão próximos à imagem, ou seja, a solução linear do sistema de equações $\mathbf{A} \mathbf{f}=\mathbf{0}$ desloca os epipolos em direção ao centro de imagem. A introdução do fator de escalonamento $k$ diminui o efeito do ruído na determinação da matriz fundamental, e a normalização torna o algoritmo invariante em relação a escala e a escolha da origem do sistema de coordenadas, determinando um sistema de coordenadas canônico.

Desta forma, uma normalização conveniente consiste na translação e escalonamento de cada imagem de forma que o centróide dos pontos seja deslocado para a origem do sistema de coordenadas de imagem e a distância quadrática média (valor RMS - vide Seção A.7) destes pontos em relação à origem seja $\sqrt{2}$. Nestas condições, dado um conjunto de $N$ pares de pontos correspondentes $\mathbf{x}_{i} \leftrightarrow \mathbf{x}_{i}^{\prime}$, com $\mathbf{x}_{i}=\left(x_{i}, y_{i}, 1\right)^{\mathrm{T}}$ e $\mathbf{x}_{i}^{\prime}=\left(x_{i}^{\prime}, y_{i}^{\prime}, 1\right)^{\mathrm{T}}$, e considerando a origem dos sistemas de coordenadas da primeira e da segunda imagem respectivamente em $\mathbf{x}_{0}=\left(x_{0}, y_{0}, 1\right)^{\mathrm{T}}$ e $\mathbf{x}_{0}^{\prime}=\left(x_{0}^{\prime}, y_{0}^{\prime}, 1\right)^{\mathrm{T}}$, as transformações de normalização da primeira e da segunda imagem são representadas respectivamente pelas matrizes $\mathrm{T}$ e $\mathrm{T}^{\prime}$ a seguir:

$$
\mathrm{T}=\left[\begin{array}{ccc}
\frac{\sqrt{2 N}}{\sum_{i} \sqrt{\left(x_{i}-x_{0}\right)^{2}+\left(y_{i}-y_{0}\right)^{2}}} & 0 & -\frac{\sum_{i}\left(x_{i}-x_{0}\right)}{N} \\
0 & \frac{\sqrt{2 N}}{\sum_{i} \sqrt{\left(x_{i}-x_{0}\right)^{2}+\left(y_{i}-y_{0}\right)^{2}}} & -\frac{\sum_{i}\left(y_{i}-y_{0}\right)}{N} \\
0 & 0 & 1
\end{array}\right],
$$




$$
\mathrm{T}^{\prime}=\left[\begin{array}{ccc}
\frac{\sqrt{2 N}}{\sum_{i} \sqrt{\left(x_{i}^{\prime}-x_{0}^{\prime}\right)^{2}+\left(y_{i}^{\prime}-y_{0}^{\prime}\right)^{2}}} & 0 & -\frac{\sum_{i}\left(x_{i}^{\prime}-x_{0}^{\prime}\right)}{N} \\
0 & \frac{\sqrt{2 N}}{\sum_{i} \sqrt{\left(x_{i}^{\prime}-x_{0}^{\prime}\right)^{2}+\left(y_{i}^{\prime}-y_{0}^{\prime}\right)^{2}}} & -\frac{\sum_{i}\left(y_{i}^{\prime}-y_{0}^{\prime}\right)}{N} \\
0 & 0 & 1
\end{array}\right]
$$

\section{A.7 $\quad$ Valor RMS}

O valor quadrático médio, ou valor RMS (do inglês Root Mean Square), também chamado de valor eficaz, é uma medida estatística da magnitude de uma quantidade variável, determinado pela raiz quadrada da média aritmética dos quadrados dos valores de uma série discreta ou de uma função contínua. O valor RMS para uma coleção de $N$ valores $\left\{x_{1}, x_{2}, \ldots, x_{N}\right\}$ é dado por:

$$
x_{\mathrm{RMS}}=\sqrt{\frac{1}{N} \sum_{i=1}^{N} x_{i}^{2}} .
$$

\section{A.8 Algoritmo DLT}

Conforme (HARTLEY; ZISSERMAN, 2000), o algoritmo DLT - Direct Linear Transformation - é um método simples de determinar uma transformação $2 \mathrm{D} \mathrm{H}$ a partir de um conjunto de quatro pares de pontos correspondentes, $\mathbf{x}_{i} \leftrightarrow \mathbf{x}_{i}^{\prime}$, tais que $\mathbf{x}_{i}^{\prime}=\mathrm{Hx}_{i}$. No entanto, o algoritmo DLT pode ser facilmente estendido para outras situações, como a triangulação de pontos correspondentes entre imagens a partir das matrizes de câmera.

A relação $\mathbf{x}_{i}^{\prime}=\mathrm{Hx}_{i}$ pode ser expressa em termos do produto vetorial como $\mathbf{x}_{i}^{\prime} \times \mathrm{Hx}_{i}=\mathbf{0}$. Esta forma permite encontrar uma solução linear para a matriz $\mathrm{H}$. Denotando a $j$-ésima linha de $\mathrm{H}$ por $\mathbf{h}^{j^{\mathrm{T}}}$, tem-se:

$$
\mathrm{Hx}_{i}=\left(\begin{array}{c}
\mathbf{h}^{1^{\mathrm{T}}} \mathbf{x}_{i} \\
\mathbf{h}^{2^{\mathrm{T}} \mathbf{x}_{i}} \\
\mathbf{h}^{3^{\mathrm{T}} \mathbf{x}_{i}}
\end{array}\right)
$$

Considerando $\mathbf{x}_{i}^{\prime}=\left(x_{i}^{\prime}, y_{i}^{\prime}, w_{i}^{\prime}\right)^{\mathrm{T}}$, o produto vetorial é dado por: 


$$
\mathbf{x}_{i}^{\prime} \times H \mathbf{x}_{i}=\left(\begin{array}{c}
y_{i}^{\prime} \mathbf{h}^{3^{\mathrm{T}}} \mathbf{x}_{i}-w_{i}^{\prime} \mathbf{h}^{2^{\mathrm{T}}} \mathbf{x}_{i} \\
w_{i}^{\prime} \mathbf{h}^{1^{\mathrm{T}}} \mathbf{x}_{i}-x_{i}^{\prime} \mathbf{h}^{3^{\mathrm{T}}} \mathbf{x}_{i} \\
x_{i}^{\prime} \mathbf{h}^{2^{\mathrm{T}}} \mathbf{x}_{i}-y_{i}^{\prime} \mathbf{h}^{1^{\mathrm{T}}} \mathbf{x}_{i}
\end{array}\right)
$$

Como $\mathbf{h}^{j^{\mathrm{T}}} \mathbf{x}_{i}=\mathbf{x}_{i}^{\mathrm{T}} \mathbf{h}^{j}$ para $j=1, \ldots, 3$, obtém-se um conjunto de três equações sobre os elementos de $\mathrm{H}$, dado por:

$$
\left[\begin{array}{ccc}
\mathbf{0}^{\mathrm{T}} & -w_{i}^{\prime} \mathbf{x}_{i}^{\mathrm{T}} & y_{i}^{\prime} \mathbf{x}_{i}^{\mathrm{T}} \\
w_{i}^{\prime} \mathbf{x}_{i}^{\mathrm{T}} & \mathbf{0}^{\mathrm{T}} & -x_{i}^{\prime} \mathbf{x}_{i}^{\mathrm{T}} \\
-y_{i}^{\prime} \mathbf{x}_{i}^{\mathrm{T}} & x_{i}^{\prime} \mathbf{x}_{i}^{\mathrm{T}} & \mathbf{0}^{\mathrm{T}}
\end{array}\right]\left(\begin{array}{c}
\mathbf{h}^{1} \\
\mathbf{h}^{2} \\
\mathbf{h}^{3}
\end{array}\right)=\mathbf{0} .
$$

Estas equações têm a forma $\mathrm{A}_{i} \mathbf{h}=0$, onde $\mathrm{A}_{i}$ é uma matriz $3 \times 9$, e $\mathbf{h}=$ $\left(\mathbf{h}^{1^{\mathrm{T}}}, \mathbf{h}^{2^{\mathrm{T}}}, \mathbf{h}^{3^{\mathrm{T}}}\right)^{\mathrm{T}}$ é um vetor de 9 elementos formado pelos elementos de H. Observase que apenas duas das linhas de $\mathrm{A}_{i}$ são linearmente independentes, de forma que o sistema pode ser simplificado para:

$$
\mathrm{A}_{i} \mathbf{h}=\left[\begin{array}{ccc}
\mathbf{0}^{\mathrm{T}} & -w_{i}^{\prime} \mathbf{x}_{i}^{\mathrm{T}} & y_{i}^{\prime} \mathbf{x}_{i}^{\mathrm{T}} \\
w_{i}^{\prime} \mathbf{x}_{i}^{\mathrm{T}} & \mathbf{0}^{\mathrm{T}} & -x_{i}^{\prime} \mathbf{x}_{i}^{\mathrm{T}}
\end{array}\right]\left(\begin{array}{c}
\mathbf{h}^{1} \\
\mathbf{h}^{2} \\
\mathbf{h}^{3}
\end{array}\right)=\mathbf{0}
$$

Cada par de pontos correspondentes $\mathbf{x}_{i} \leftrightarrow \mathbf{x}_{i}^{\prime}$ determina duas equações sobre os elementos de H. Dado um conjunto de $n \geq 4$ quatro pares de pontos correspondentes, determina-se um sistema de equações $\mathbf{A h}=\mathbf{0}$, onde $\mathbf{A}=\left[\mathrm{A}_{i}\right]$ é uma matriz $2 n \times 9$ representando o conjunto completo de equações. A solução deste sistema pode ser obtida através da SVD da matriz A, e $\mathbf{h}$ é dado pelo vetor singular correspondente ao menor valor singular de A.

\section{A.9 Complexidade Computacional}

Conforme (LEWIS; PAPADIMITRIOU, 1997), a complexidade computacional de um algoritmo consiste no custo de sua execução, expresso em função das operações fundamentais, que variam de acordo com o algoritmo, e em função do volume de dados. É medida segundo um modelo matemático que supõe que o algoritmo possui uma entrada (massa de dados) de tamanho $N$. O processo de execução de um algoritmo pode ser dividido em etapas elementares denominadas passos, e o número de passos de um algoritmo corresponde ao número de execuções de uma operação dominante em função das entradas, desprezando-se constantes aditivas ou multiplicativas. A complexidade computacional analisa o grau de dificuldade 
de um algoritmo em termos de diferentes recursos computacionais - como tempo, espaço, aleatoriedade, alternância e outras medidas menos intuitivas. Uma classe de complexidade é o conjunto de todos algoritmos que podem ser solucionados utilizando-se uma certa quantidade de um dado recurso computacional. Os recursos computacionais mais comumente utilizados em complexidade computacional são tempo desterminístico e espaço determinístico, representando o tempo computacional e o espaço de memória necessário para execução do algoritmo em hadware determinístico (como é o caso dos computadores disponíveis na atualidade). A complexidade computacional de um algoritmo é dada em função do tamanho do conjunto de dados de entrada e do número de passos deste algoritmo. Para um conjunto de dados de entrada de tamanho $n$, um algoritmo com $n^{2}$ passos apresenta complexidade $n^{2}$. O número exato de passos depende do hardware e da linguagem de programação, entre outros fatores. Para eliminar estas dependências, introduz-se a notação $\mathcal{O}(\cdot)(B i g-O h)$. Através desta notação, um algoritmo de complexidade $\mathcal{O}\left(n^{2}\right)$ apresenta o mesmo custo em qualquer hardware em que seja executado. A notação $\mathcal{O}(\cdot)$ expressa comparativamente o crescimento assintótico de duas funções, e representa a pers-pectiva de pior caso da complexidade computacional de um algoritmo - definindo uma classe de complexidade. Por definição, $\mathbf{f}=\mathcal{O}(\mathrm{g})$ se existe uma constante $c>0$ e um valor $n_{0}$ tal que para $n>n_{0}, \mathrm{f}(n) \leq \mathrm{cg}(n)$. Ou seja, a função g atua como limite superior para valores assintóticos da função $f$. Segundo esta definição, valem as propriedades $\mathcal{O}(\mathrm{f}+\mathrm{g})=\mathcal{O}(\mathrm{f})+\mathcal{O}(\mathrm{g})$ e $\mathcal{O}(K \mathrm{f})=K \mathcal{O}(\mathrm{f})$. Normalmente expressa-se a complexidade computacional como uma combinação de funções elementares: constante unitária, polinomiais $\left(n, n^{2}\right.$ etc.), $\log _{2}(n), 2^{n}$ entre outras. 


\section{Apêndice B - Listagens dos Programas}

Os programas apresentados nas próximas seções foram desenvolvidos em MATLAB ${ }^{\circledR}$

6.5, linguagem de alto desempenho voltada ao cálculo numérico e matricial, amplamente utilizada em aplicações científicas e de engenharia, adotada para todos os cálculos deste trabalho.

\section{B.1 Cálculo da Matriz Fundamental}

\section{B.1.1 Algoritmo de 7 Pontos Correspondentes}

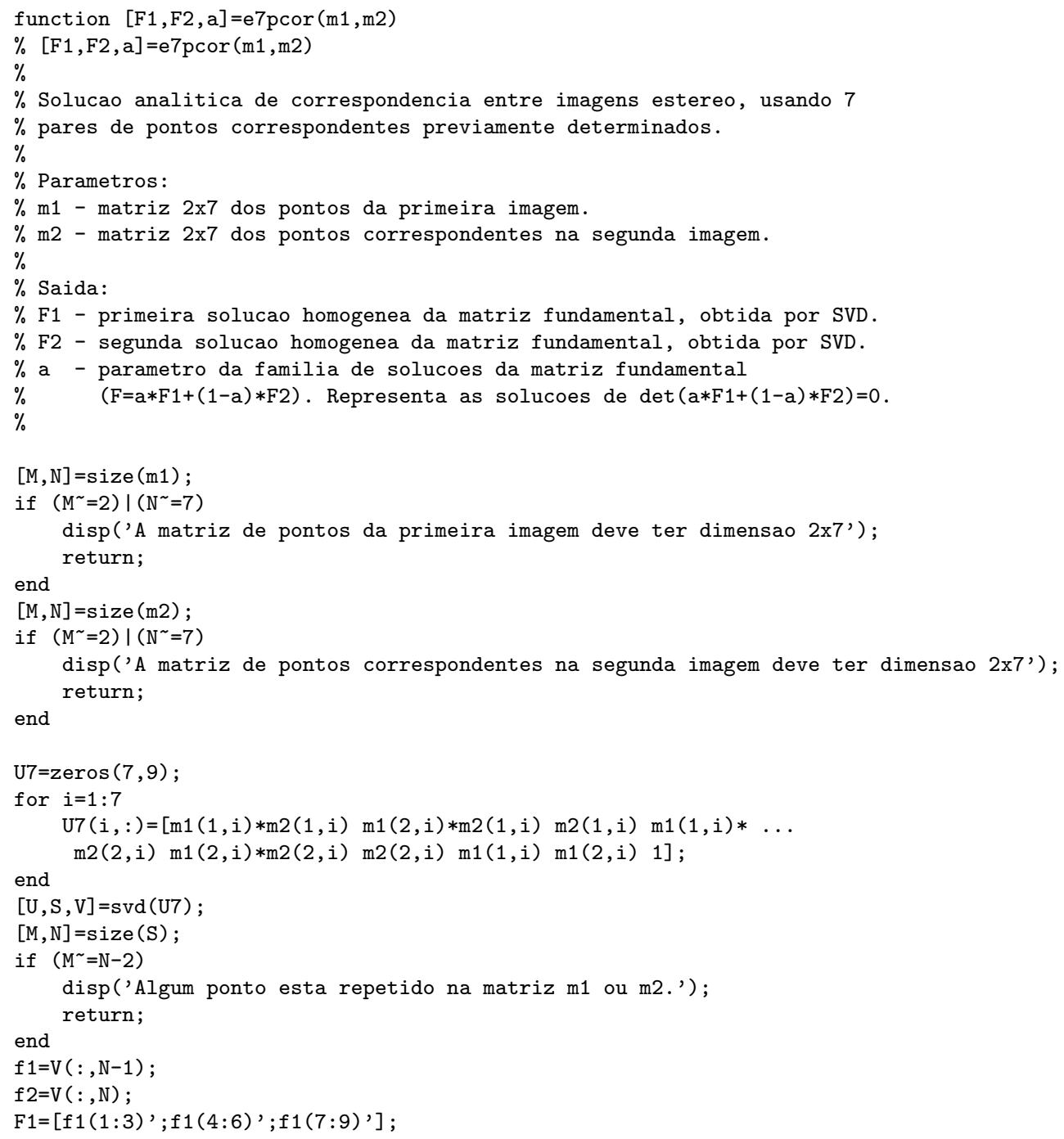




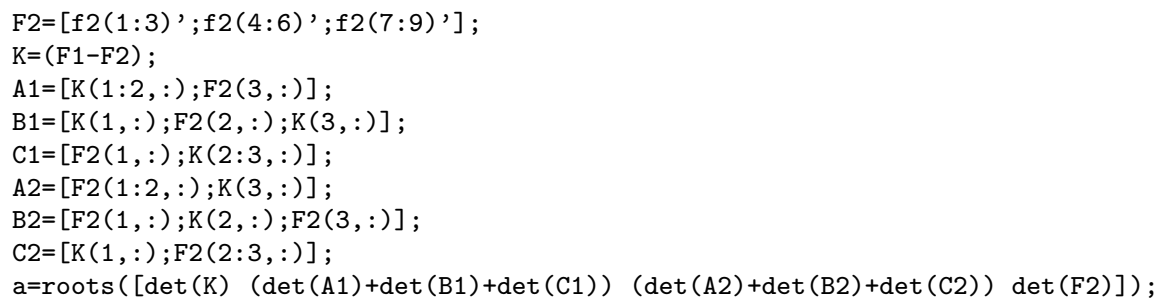

\section{B.1.2 Algoritmo de 8 Pontos Correspondentes Norma- lizado}

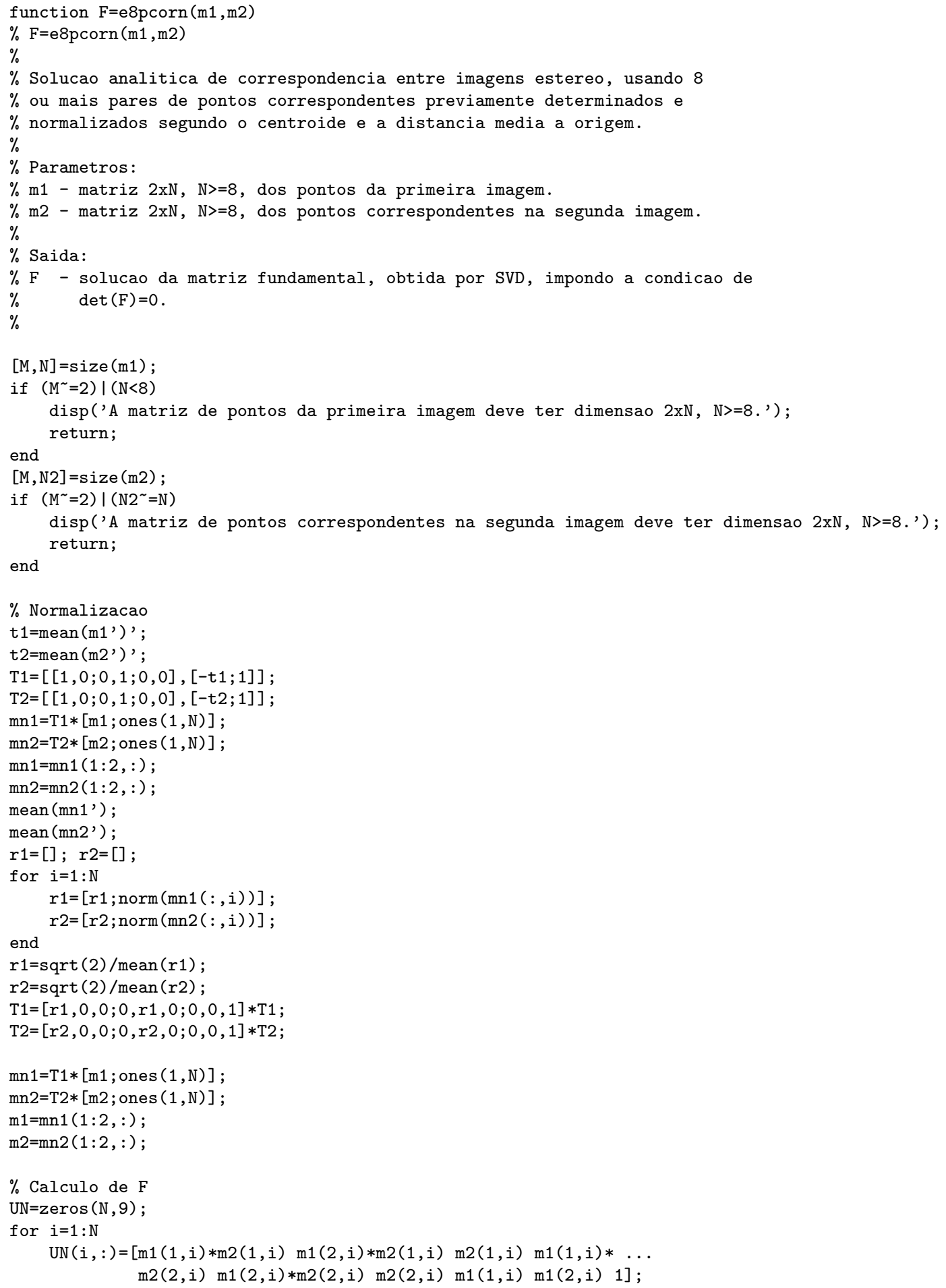


end

$[\mathrm{U}, \mathrm{S}, \mathrm{V}]=\mathrm{svd}(\mathrm{UN})$

$[\mathrm{J}, \mathrm{K}]=\operatorname{size}(\mathrm{S})$;

$\mathrm{f} 1=\mathrm{V}(:, \mathrm{K})$;

$F=\left[f 1(1: 3)^{\prime} ; f 1(4: 6)^{\prime} ; f 1(7: 9)^{\prime}\right]$;

$[\mathrm{U}, \mathrm{S}, \mathrm{V}]=\operatorname{svd}(\mathrm{F})$;

$\mathrm{d}=\operatorname{diag}(\mathrm{S})$;

$\mathrm{d}=[\mathrm{d}(1: \operatorname{size}(\mathrm{d})-1) ; 0]$;

$\mathrm{F}=\mathrm{U} * \operatorname{diag}(\mathrm{d}) * \mathrm{~V}^{\prime}$;

\% Desnormalizacao

$\mathrm{F}=\mathrm{T} 2{ }^{\prime} * \mathrm{~F} * \mathrm{~T} 1$

\section{B.1.3 Algoritmo de Busca Exaustiva}

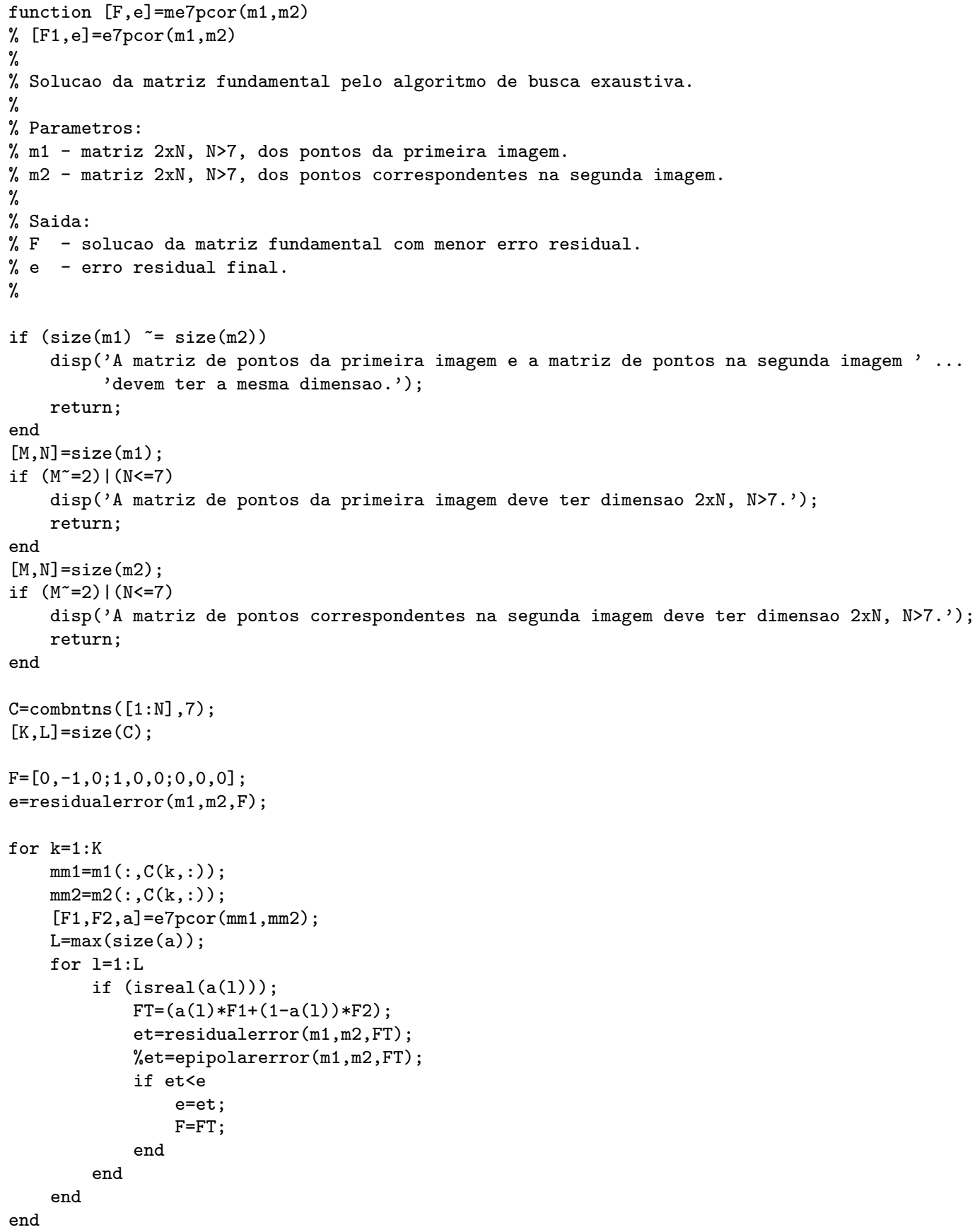




\section{B.1.4 Algoritmo Linear Iterativo}

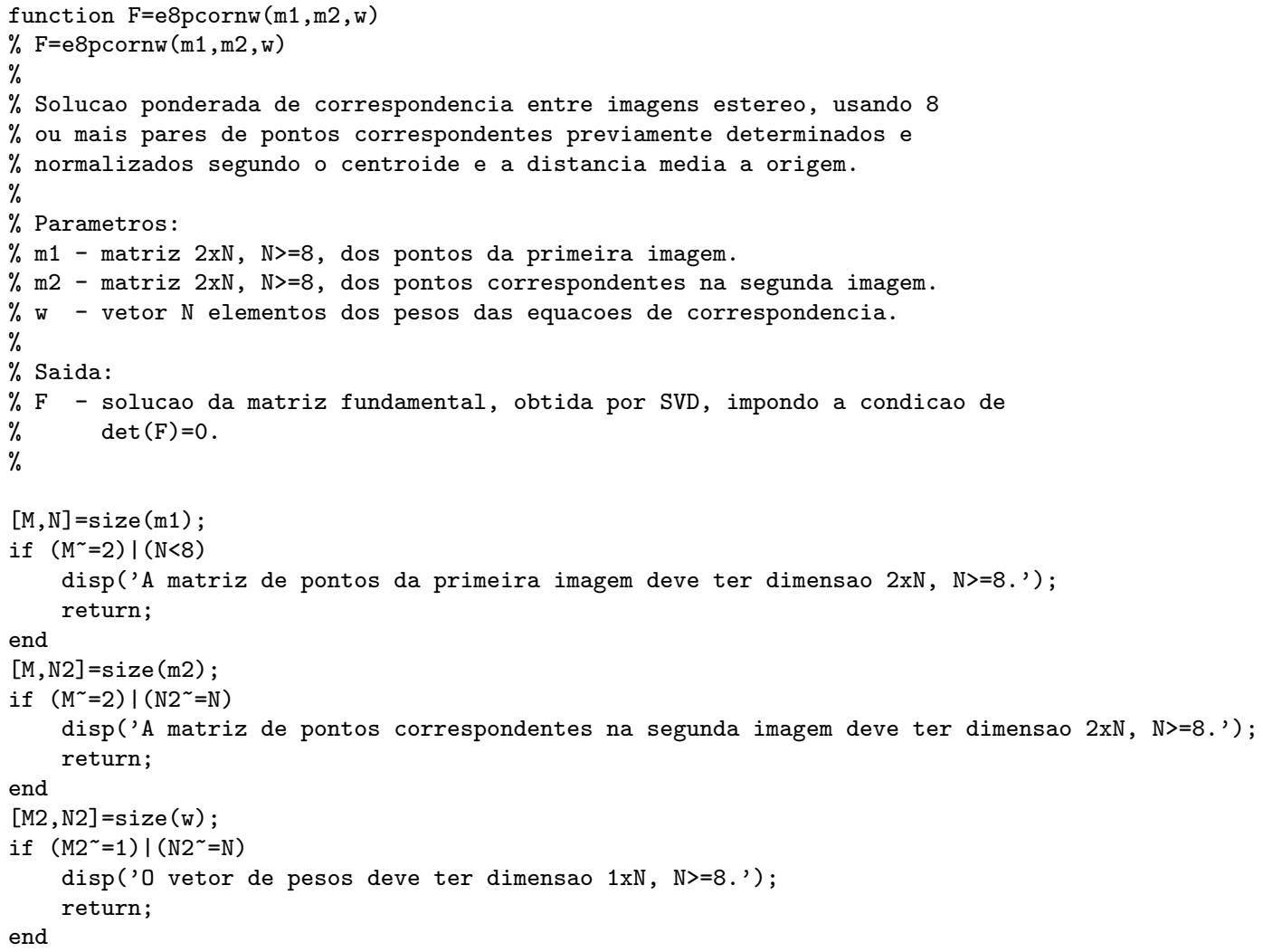




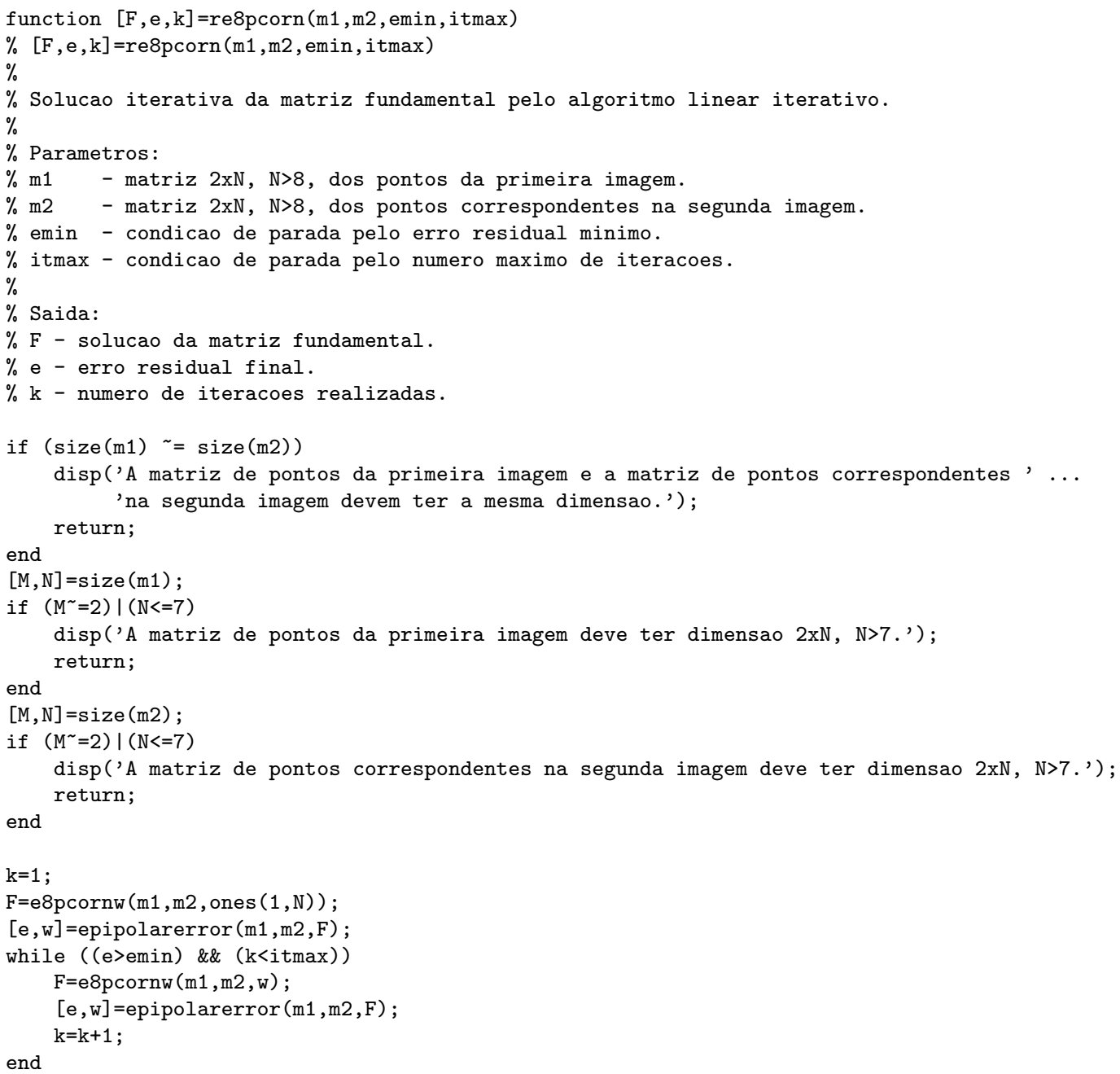

\section{B.2 Matrizes de Projeção Canônicas}

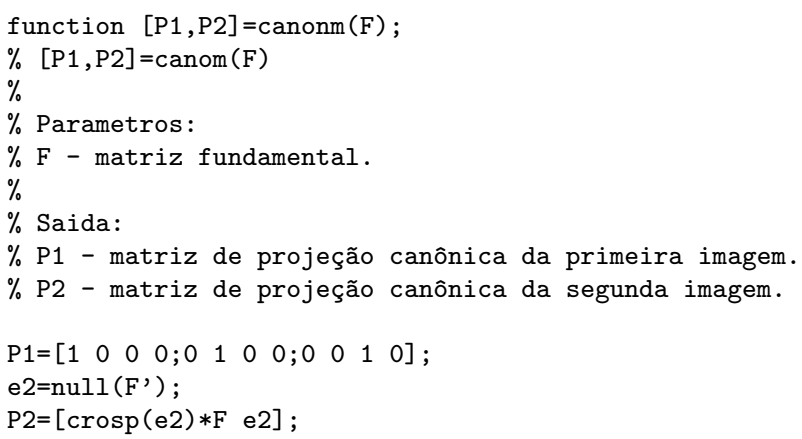

\section{B.3 Triangulação}

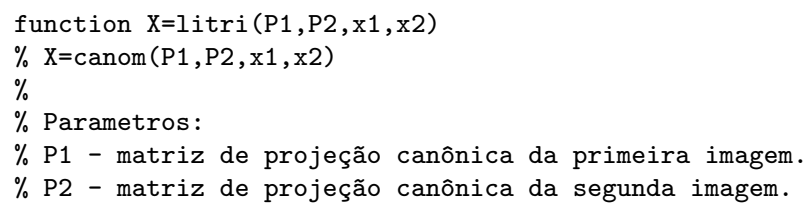




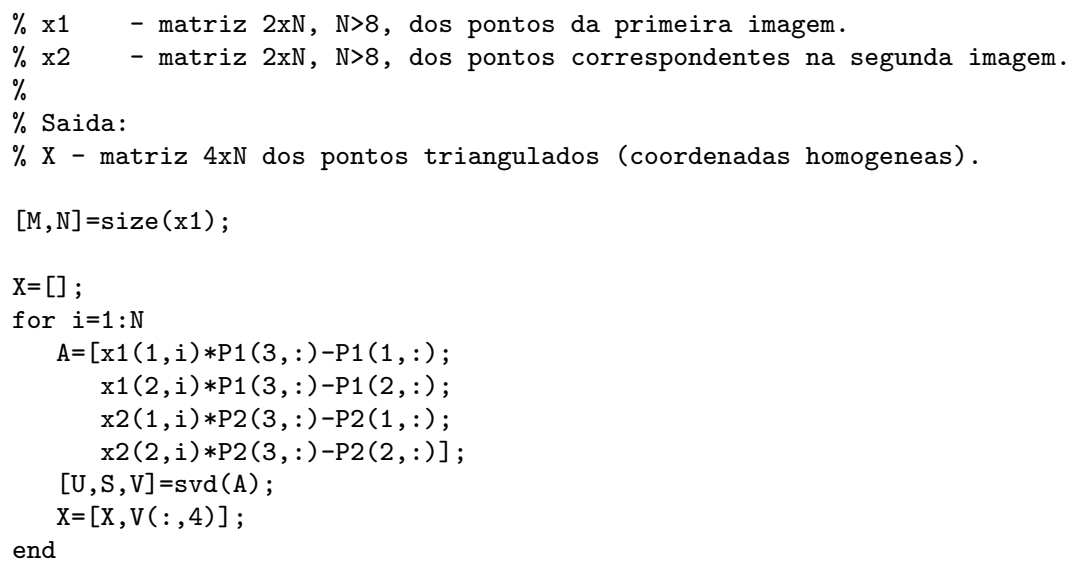

\section{B.4 Matrizes de Projeção Métricas}

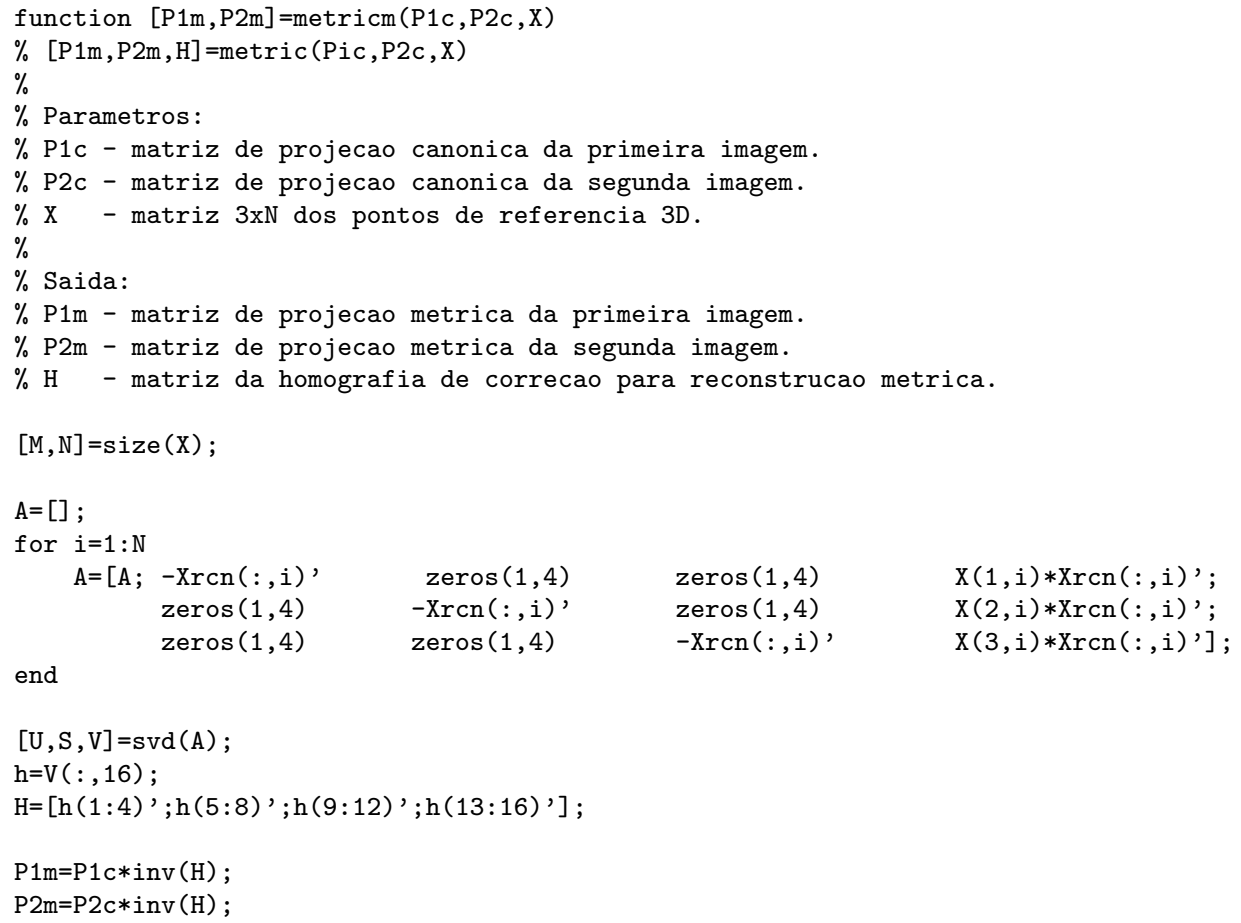

\section{B.5 Erros}

\section{B.5.1 Erro Residual}

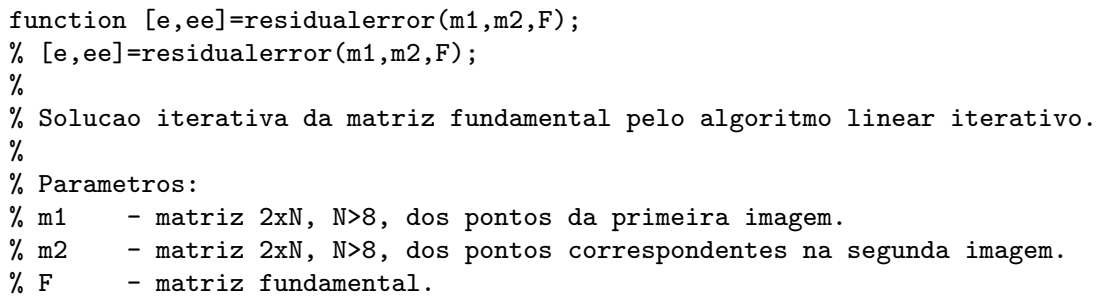




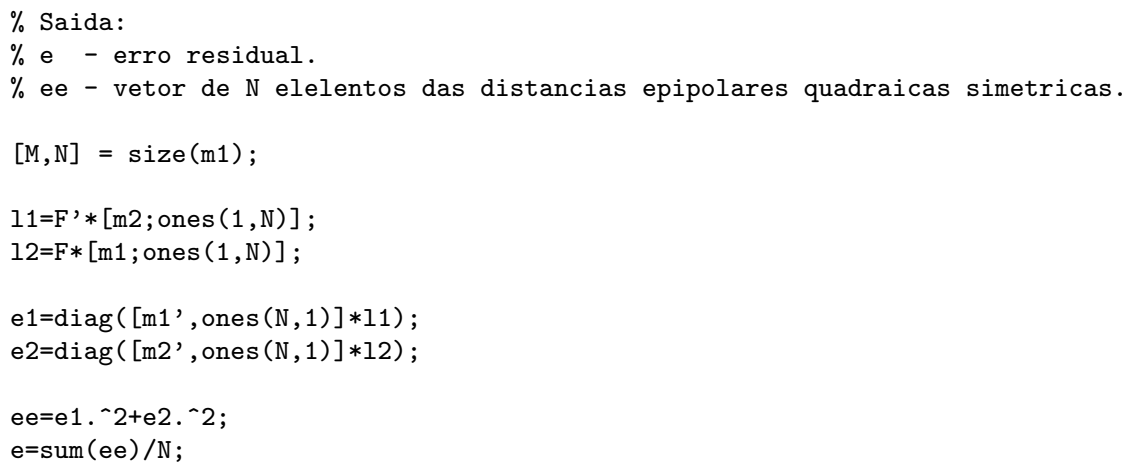

\section{B.5.2 Erro de Reconstrução}

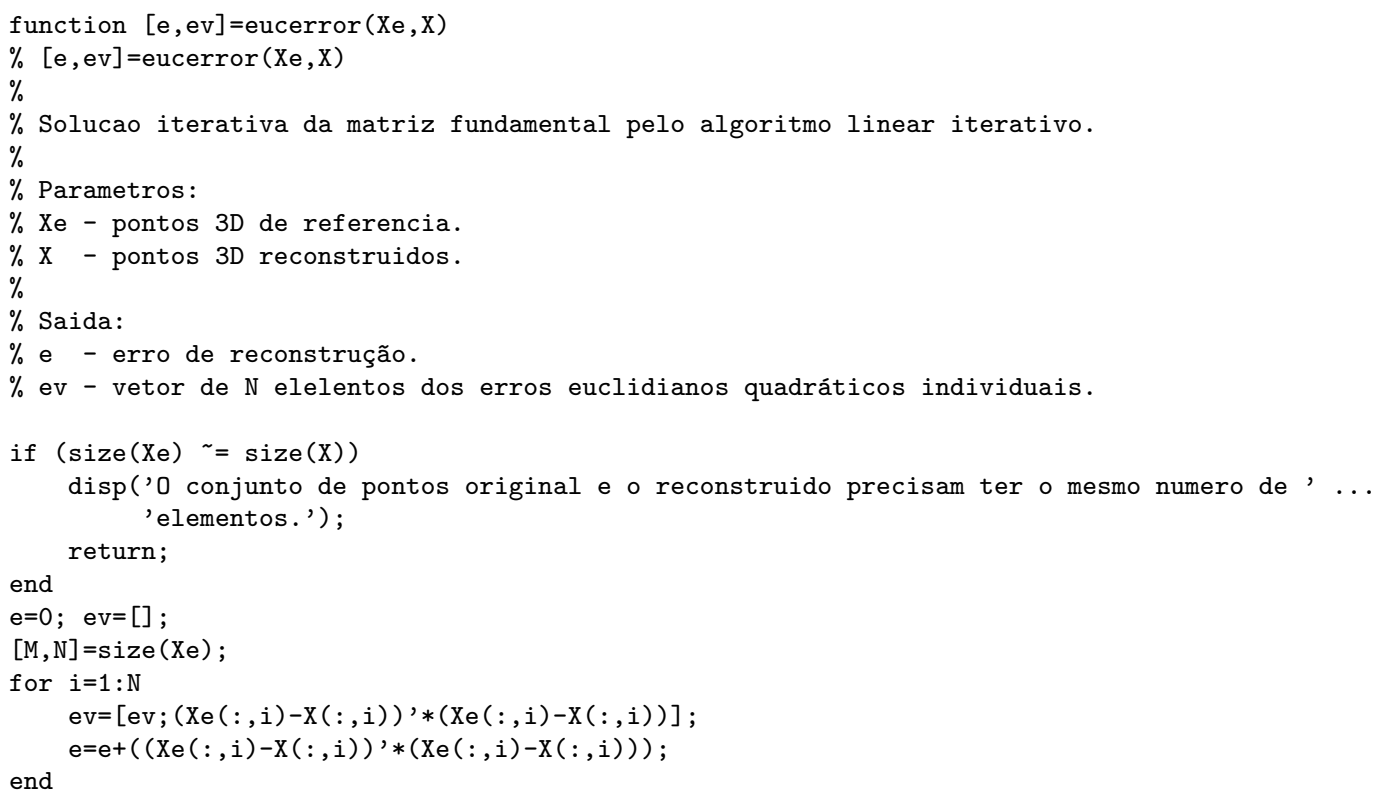

\section{B.6 Interface Gráfica}

function varargout = correspondence (varargin) $\%$ CORRESPONDENCE M-file for correspondence.fig CORRESPONDENCE, by itself, creates a new CORRESPONDENCE or raises the existing singleton*.

$\mathrm{H}=$ CORRESPONDENCE returns the handle to a new CORRESPONDENCE or the handle to the existing singleton*.

CORRESPONDENCE ('CALLBACK', hObject, eventData, handles,...) calls the local function named CALLBACK in CORRESPONDENCE.M with the given input arguments.

CORRESPONDENCE ('Property', 'Value',...) creates a new CORRESPONDENCE or raises the existing singleton*. Starting from the left, property value pairs are applied to the GUI before correspondence_OpeningFunction gets called. An unrecognized property name or invalid value makes property application stop. All inputs are passed to correspondence_OpeningFen via varargin.

*See GUI Options on GUIDE's Tools menu. Choose "GUI allows only one instance to run (singleton)".

See also: GUIDE, GUIDATA, GUIHANDLES 
$\%$ Edit the above text to modify the response to help correspondence

\% Last Modified by GUIDE v2.5 11-Jan-2005 12:02:16

$\%$ Begin initialization code - DO NOT EDIT

gui_Singleton $=1$;

gui_State $=$ struct ('gui_Name', mfilename, $\ldots$.

'gui_Singleton', gui_Singleton, ...

'gui_OpeningFcn', @correspondence_OpeningFcn, ...

'gui_OutputFcn', @correspondence_OutputFcn, ...

'gui_LayoutFcn', [], ...

'gui_Callback', []);

if nargin \& isstr (varargin\{1\})

end

gui_State.gui_Callback $=\operatorname{str} 2 f$ unc $(\operatorname{varargin}\{1\})$;

if nargout

else

[varargout $\{1:$ nargout $\}]=$ gui_mainf cn (gui_State, $\operatorname{varargin}\{:\})$;

end

gui_mainfcn (gui_State, varargin $\{:\})$;

$\%$ End initialization code - DO NOT EDIT

\% --- Executes just before correspondence is made visible.

function correspondence_OpeningFcn(hObject, eventdata, handles, varargin)

$\%$ This function has no output args, see OutputFcn.

$\%$ hObject handle to figure

$\%$ eventdata reserved - to be defined in a future version of MATLAB

$\%$ handles structure with handles and user data (see GUIDATA)

$\%$ varargin command line arguments to correspondence (see VARARGIN)

\% Choose default command line output for correspondence

handles . output $=$ hObject;

$\%$ Update handles structure

guidata(hObject, handles);

$\%$ UIWAIT makes correspondence wait for user response (see UIRESUME)

$\%$ uiwait (handles.figure1);

$\%$--- Outputs from this function are returned to the command line.

function varargout $=$ correspondence_OutputFcn(hObject, eventdata, handles)

$\%$ varargout cell array for returning output args (see VARARGOUT);

$\%$ hObject handle to figure

$\%$ eventdata reserved - to be defined in a future version of MATLAB

$\%$ handles structure with handles and user data (see GUIDATA)

$\%$ Get default command line output from handles structure

varargout $\{1\}=$ handles.output;

$\%$--- Executes on button press in load_fig1.

function load_im1_Callback(hObject, eventdata, handles)

$\%$ hObject handle to load_fig1 (see GCBO)

$\%$ eventdata reserved - to be defined in a future version of MATLAB

$\%$ handles structure with handles and user data (see GUIDATA)

[filename, pathname] $=$ uigetfile $(\ldots$

$\{' *$.bmp;*.cur;*.gif;*.hdf;*.ico;*.jpg;*.jpeg;*.pbm;*.pcx;*.pgm;*.png;*.ppm;' ...

'*.ras;*.tif;*.tiff;*.xwd', 'All supported formats'; ...

'*.bmp', 'BMP (Microsoft Windows Bitmap)'; ...

'*.cur', 'CUR (Microsoft Windows Cursor resource)'; ...

'*.gif', 'GIF (Graphics Interchange Format)'; ...

'*.hdf', 'HDF (Hierarchical Data Format)'; ...

'*.ico', 'ICO (Windows Icon resource)'; ...

'*.jpg;*.jpeg', 'JPEG (Joint Photographic Experts Group)'; ...

'*.pbm', 'PBM (Portable Bitmap)'; ...

'*.pcx', 'PCX (Windows Paintbrush)'; ...

'*.pgm', 'PGM (Portable Graymap)'; ...

'*.png', 'PNG (Portable Network Graphics)'; ...

'*.ppm', 'PPM (Portable Pixmap)'; ...

'*.ras', 'RAS (Sun Raster image)'; ...

'*.tif;*.tiff', 'TIFF (Tagged Image File Format)'; ...

'*. xwd', 'XWD (X Window Dump)'; ...

'*.*', 'All Files $\left.(* . *)^{\prime}\right\}, \ldots$

'Load Image 1');

if isstr(filename) \&\& isstr (pathname)

handles.im1 = imread ([pathname filename $])$; 
if isrgb(handles.im1)

handles.gim1 = rgb2gray (handles.im1);

elseif isind(handles.im1) || isgray(handles.im1)

end

handles.gim1 = handles.im1;

$[\mathrm{M}, \mathrm{N}, \mathrm{T}]=\operatorname{size}($ handles. $\mathrm{im} 1)$;

handles. $\mathrm{X} 10=$ round $(\mathrm{N} / 2)$;

handles . Y10=round $(M / 2)$;

handles.npoints $=0$;

handles.pts1= [];

handles.pts2=[];

axes (handles.fig1);

axis image;

imshow (handles.im1);

set (handles.gray_im1, 'Enable', 'on') ;

set (handles.edge_im1, 'Enable', 'on') ;

set (handles.zoom_im1, 'Enable', 'on');

set (handles.reset_im1, 'Enable', 'on') ;

set (handles.collect_im1, 'Enable', 'on') ;

guidata(hObject, handles);

set (handles.gray_im1, 'Value', get (handles.gray_im1, 'Min')) ;

set (handles.edge_im1, 'Value', get (handles.edge_im1, 'Min'));

set (handles.zoom_im1, 'Value', get (handles.zoom_im1, 'Min')) ;

end

guidata(hObject, handles);

$\%$--- Executes on button press in load_im2.

function load_im2_Callback(hObject, eventdata, handles)

$\%$ hObject handle to load_im2 (see GCBO)

$\%$ eventdata reserved - to be defined in a future version of MATLAB

$\%$ handles structure with handles and user data (see GUIDATA)

[filename, pathname] $=\operatorname{uigetfile}(\ldots$

$\left\{{ }^{\prime} *\right.$.bmp;*.cur;*.gif;*.hdf;*.ico;*.jpg;*.jpeg;*.pbm;*.pcx;*.pgm;*.png;*.ppm;' ...

'*.ras;*.tif;*.tiff;*.xwd', 'All supported formats'; ..

'*.bmp', 'BMP (Microsoft Windows Bitmap)'; ...

'*.cur', 'CUR (Microsoft Windows Cursor resource)'; ...

'*.gif', 'GIF (Graphics Interchange Format)'; ...

'*.hdf', 'HDF (Hierarchical Data Format)'; ...

'*.ico', 'ICO (Windows Icon resource)'; ...

'*.jpg;*.jpeg', 'JPEG (Joint Photographic Experts Group)'; ...

'*.pbm', 'PBM (Portable Bitmap)'; ...

'*.pcx', 'PCX (Windows Paintbrush)'; ...

'*.pgm', 'PGM (Portable Graymap)'; ...

'*.png', 'PNG (Portable Network Graphics)'; ...

'*.ppm', 'PPM (Portable Pixmap)'; ...

'*.ras', 'RAS (Sun Raster image)'; ...

'*.tif;*.tiff', 'TIFF (Tagged Image File Format)'; ...

'*.xwd', 'XWD (X Window Dump)'; ...

'*.*', 'All Files $(* . *)$ '\}, ...

'Load Image 2')

if isstr(filename) \&\& isstr(pathname)

handles.im2 = imread ([pathname filename]);

if isrgb(handles.im2)

handles.gim2 = rgb2gray(handles.im2);

elseif isind(handles.im2) || isgray(handles.im2)

handles.gim2 = handles.im2;

end

$[\mathrm{M}, \mathrm{N}, \mathrm{T}]=\operatorname{size}($ handles . im2);

handles . X20=round $(N / 2)$;

handles . Y20=round $(M / 2)$;

handles.npoints $=0$;

handles.pts1= [] ;

handles.pts $2=[]$;

axes (handles.fig2);

axis image;

imshow (handles.im2);

set (handles.gray_im2, 'Enable', 'on') ;

set (handles.edge_im2, 'Enable', 'on');

set (handles.zoom_im2, 'Enable', 'on');

set (handles.reset_im2, 'Enable', 'on');

set (handles.collect_im2, 'Enable', 'on') ;

guidata(hObject, handles);

set (handles.gray_im2, 'Value', get (handles.gray_im2, 'Min')) ;

set (handles.edge_im2, 'Value', get (handles.edge_im2, 'Min')) ;

set (handles.zoom_im2, 'Value', get (handles.zoom_im2, 'Min')) ;

guidata(hObject, handles); 


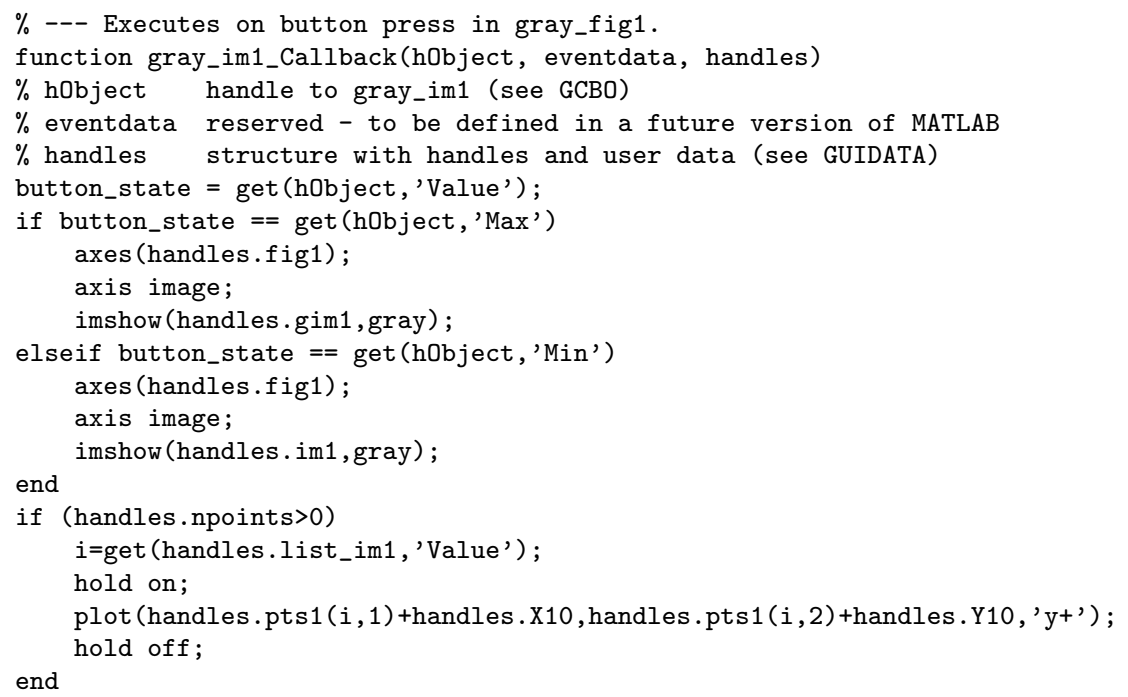

$\%$--- Executes on button press in gray_im2.

function gray_im2_Callback(hObject, eventdata, handles)

$\%$ hObject handle to gray_im2 (see GCBO)

$\%$ eventdata reserved - to be defined in a future version of MATLAB

$\%$ handles structure with handles and user data (see GUIDATA)

button_state $=$ get $($ hObject, 'Value') ;

if button_state $==$ get (hObject, 'Max')

axes (handles.fig2);

axis image;

imshow (handles.gim2);

elseif button_state $==\operatorname{get}($ hObject, 'Min')

axes (handles.fig2);

axis image;

end

imshow (handles.im2);

if (handles.npoints $>0$ )

$i=$ get (handles.list_im2, 'Value');

hold on;

plot (handles.pts2 (i,1)+handles.X20, handles.pts2 (i, 2)+handles. Y20, ' $y+^{\prime}$ ) ;

hold off;

end

$\%$--- Executes on button press in edge_fig1.

function edge_im1_Callback(hObject, eventdata, handles)

$\%$ hObject handle to edge_fig1 (see GCBO)

$\%$ eventdata reserved - to be defined in a future version of MATLAB

$\%$ handles structure with handles and user data (see GUIDATA)

button_state $=$ get (hObject, 'Value');

if button_state $==\operatorname{get}\left(\mathrm{hObject}\right.$, 'Max' $\left.^{\prime}\right)$

val = get(handles.filter_im1,'Value');

disp(num2str(val));

switch val

case 1

axes (handles.fig1);

axis image;

imshow (edge (handles .gim1, 'sobel'));

case 2

axes (handles.fig1);

axis image;

imshow (edge (handles .gim1, 'prewitt'));

case 3

axes (handles.fig1)

axis image;

imshow (edge (handles .gim1, 'roberts'));

case 4

axes (handles.fig1);

axis image;

imshow (edge (handles.gim1, 'log'));

case 5

axes (handles.fig1)

axis image; 


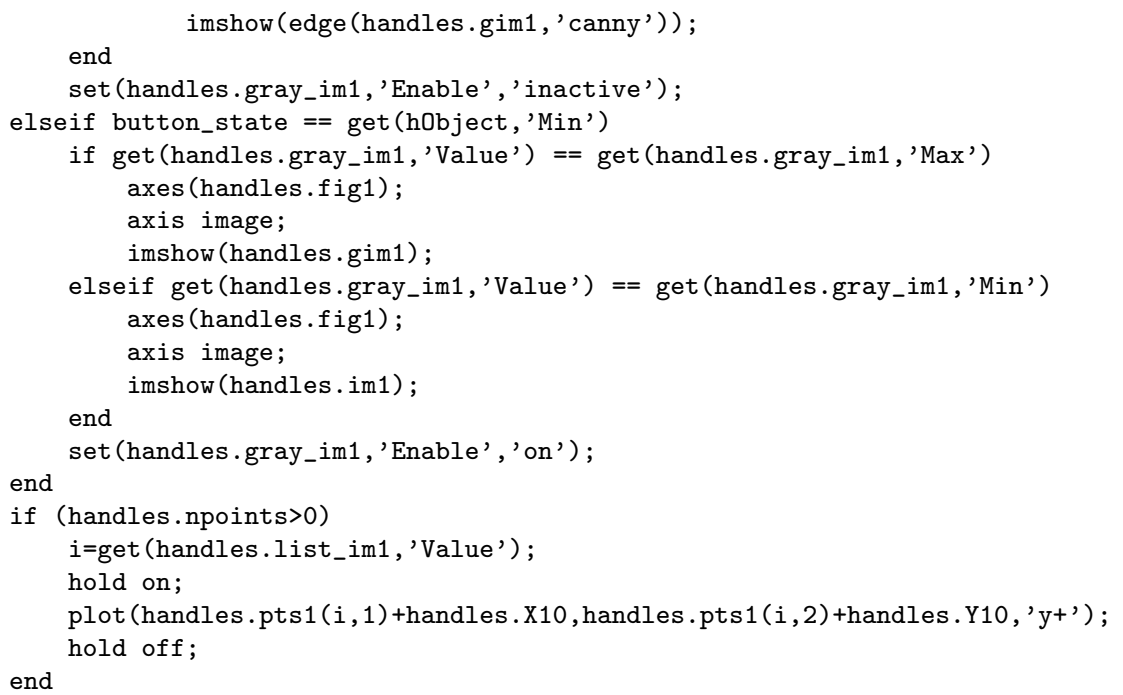




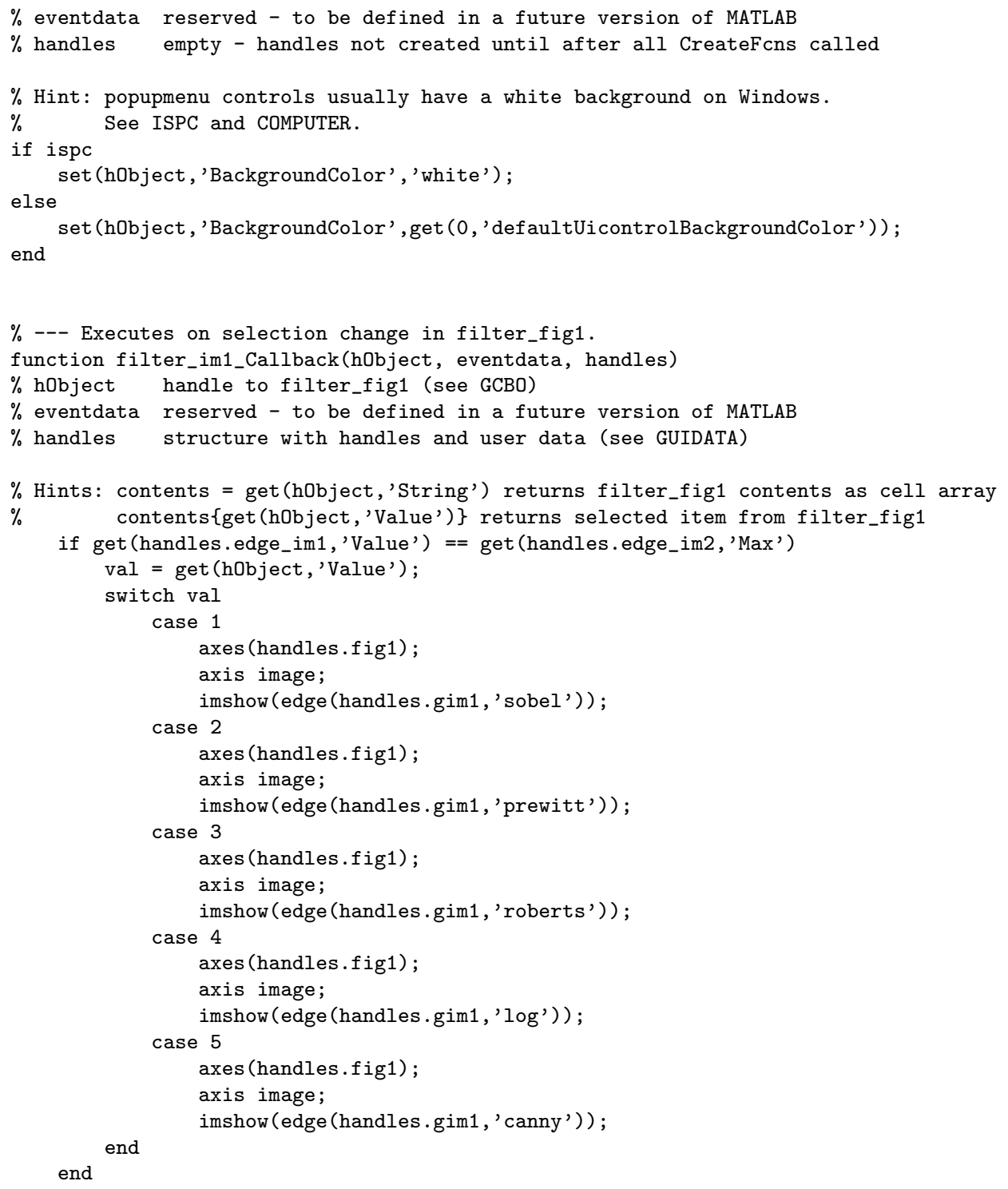

$\%$--- Executes during object creation, after setting all properties.

function filter_im2_CreateFcn(hObject, eventdata, handles)

$\%$ hObject handle to filter_im2 (see GCBO)

$\%$ eventdata reserved - to be defined in a future version of MATLAB

$\%$ handles empty - handles not created until after all CreateFcns called

$\%$ Hint: popupmenu controls usually have a white background on Windows.

$\% \quad$ See ISPC and COMPUTER.

if ispc

set (hObject, 'BackgroundColor', 'white') ;

else

set (hObject, 'BackgroundColor', get (0, 'defaultUicontrolBackgroundColor')) ;

end

$\%$--- Executes on selection change in filter_im2.

function filter_im2_Callback(hObject, eventdata, handles)

$\%$ hObject handle to filter_im2 (see GCBO)

$\%$ eventdata reserved - to be defined in a future version of MATLAB

$\%$ handles structure with handles and user data (see GUIDATA)

$\%$ Hints: contents = get(hObject,'String') returns filter_im2 contents as cell array

$\% \quad$ contents $\quad$ get (hObject, 'Value')\} returns selected item from filter_im2

if get(handles.edge_im2, 'Value') == get(handles.edge_im2, 'Max')

val = get (hObject, 'Value');

switch val

case 1

axes (handles.fig2);

axis image;

imshow (edge (handles.gim2, 'sobel')) ;

case 2 
axes (handles.fig2);

axis image;

imshow (edge (handles.gim2, 'prewitt')) ; case 3

axes (handles.fig2);

axis image;

imshow (edge(handles.gim2, 'roberts')) ;

case 4

axes (handles.fig2);

axis image;

imshow (edge (handles.gim2, 'log')) ;

case 5

axes (handles.fig2);

axis image;

end

imshow (edge (handles.gim2, 'canny')) ;

end

$\%$--- Executes on button press in zoom_im1.

function zoom_im1_Callback(hObject, eventdata, handles)

$\%$ hObject handle to zoom_im1 (see GCBO)

$\%$ eventdata reserved - to be defined in a future version of MATLAB

$\%$ handles structure with handles and user data (see GUIDATA)

\% Hint: get(hObject,'Value') returns toggle state of zoom_im1

button_state $=$ get (hObject, 'Value');

if button_state $==\operatorname{get}($ hObject, 'Max')

set (handles.load_im1, 'Enable', 'off') ;

set (handles.gray_im1, 'Enable', 'off') ;

set (handles.edge_im1, 'Enable', 'off');

set (handles.filter_im1, 'Enable', 'off') ;

set (handles.reset_im1, 'Enable', 'off') ;

set (handles.collect_im1, 'Enable', 'off');

set (handles.zoom_im2, 'Value', button_state);

set (handles.zoom_im2, 'Enable', 'off') ;

set (handles.load_im2, 'Enable', 'off') ;

set (handles.gray_im2, 'Enable', 'off');

set (handles.edge_im2, 'Enable', 'off') ;

set (handles.filter_im2, 'Enable', 'off');

set (handles.reset_im2, 'Enable', 'off');

set (handles.collect_im2, 'Enable', 'off') ;

axes (handles.fig1);

zoom on;

end

if button_state $==\operatorname{get}($ hObject, 'Min')

set (handles.load_im1, 'Enable', 'on') ;

set (handles.gray_im1, 'Enable', 'on');

set (handles.edge_im1, 'Enable', 'on');

set (handles.filter_im1, 'Enable', 'on')

set (handles.reset_im1, 'Enable', 'on') ;

set (handles.collect_im1, 'Enable', 'on') ;

set (handles.zoom_im2, 'Value', button_state);

set (handles.zoom_im2, 'Enable', 'on') ;

set (handles.load_im2, 'Enable', 'on');

set (handles.gray_im2, 'Enable', 'on');

set (handles.edge_im2, 'Enable', 'on') ;

set (handles.filter_im2, 'Enable', 'on') ;

set (handles.reset_im2, 'Enable', 'on');

set (handles.collect_im2, 'Enable', 'on') ;

axes (handles.fig1);

zoom off;

end

$\%$--- Executes on button press in zoom_im2.

function zoom_im2_Callback(hObject, eventdata, handles)

$\%$ hObject handle to zoom_im2 (see GCBO)

$\%$ eventdata reserved - to be defined in a future version of MATLAB

$\%$ handles structure with handles and user data (see GUIDATA)

\% Hint: get(hObject, 'Value') returns toggle state of zoom_im2

button_state $=$ get (hObject, 'Value');

if button_state $==\operatorname{get}($ hObject, 'Max')

set (handles.load_im2, 'Enable', 'off');

set (handles.gray_im2, 'Enable', 'off') ;

set (handles.edge_im2, 'Enable', 'off') ; 
set (handles.filter_im2, 'Enable', 'off');

set (handles.reset_im2, 'Enable', 'off') ;

set (handles.collect_im2, 'Enable', 'off') ;

set (handles.zoom_im1, 'Value', button_state);

set (handles.zoom_im1, 'Enable', 'off') ;

set (handles.load_im1, 'Enable', 'off');

set (handles.gray_im1, 'Enable', 'off') ;

set (handles.edge_im1, 'Enable', 'off');

set (handles.filter_im1, 'Enable', 'off') ;

set (handles.reset_im1, 'Enable', 'off') ;

set (handles.collect_im1, 'Enable', 'off') ;

axes (handles.fig2);

end

zoom on;

if button_state $==\operatorname{get}($ hObject, 'Min')

set (handles.load_im2, 'Enable', 'on') ;

set (handles.gray_im2, 'Enable', 'on') ;

set (handles.edge_im2, 'Enable', 'on') ;

set (handles.filter_im2, 'Enable', 'on') ;

set (handles.reset_im2, 'Enable', 'on') ;

set (handles.collect_im2, 'Enable', 'on') ;

set (handles.zoom_im1, 'Value', button_state);

set (handles.zoom_im1, 'Enable', 'on') ;

set (handles.load_im1, 'Enable', 'on') ;

set (handles.gray_im1, 'Enable', 'on');

set (handles.edge_im1, 'Enable', 'on') ;

set (handles.filter_im1, 'Enable', 'on')

set (handles.reset_im1, 'Enable', 'on') ;

set (handles.collect_im1, 'Enable', 'on') ;

axes (handles.fig2);

zoom off;

end

$\%$--- Executes on button press in reset_im1.

function reset_im1_Callback(hObject, eventdata, handles)

$\%$ hObject handle to reset_im1 (see GCBO)

$\%$ eventdata reserved - to be defined in a future version of MATLAB

$\%$ handles structure with handles and user data (see GUIDATA)

\% Hint: get(hObject,'Value') returns toggle state of reset_im1 axes (handles.fig1);

zoom out

$\%$--- Executes during object creation, after setting all properties.

function list_im1_CreateFcn(hObject, eventdata, handles)

$\%$ hObject handle to list_im1 (see GCBO)

$\%$ eventdata reserved - to be defined in a future version of MATLAB

$\%$ handles empty - handles not created until after all CreateFcns called

$\%$ Hint: listbox controls usually have a white background on Windows.

if ispc else

set (hObject, 'BackgroundColor', 'white');

end

set (hObject, 'BackgroundColor', get (0, 'defaultUicontrolBackgroundColor')) ;

$\%$--- Executes on selection change in list_im1.

function list_im1_Callback(hObject, eventdata, handles)

$\%$ hObject handle to list_im1 (see GCBO)

$\%$ eventdata reserved - to be defined in a future version of MATLAB

$\%$ handles structure with handles and user data (see GUIDATA)

$\%$ Hints: contents = get(hObject,'String') returns list_im1 contents as cell array

$i=\operatorname{get}($ hObject, 'Value');

set (handles.list_im2, 'Value',i);

button_state $=\operatorname{get}($ handles.gray_im1, 'Value');

if button_state $==$ get (handles.gray_im1, 'Max')

axes (handles.fig1);

axis image;

imshow (handles.gim1, gray);

elseif button_state $==$ get (handles.gray_im1, 'Min')

axes (handles.fig1); 


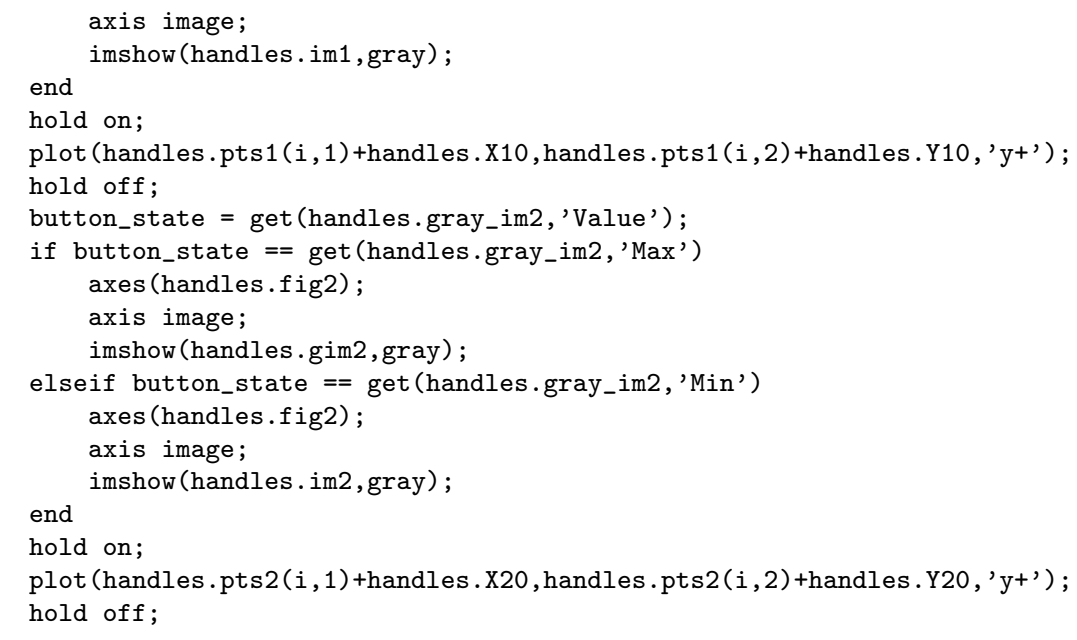

$\%$--- Executes during object creation, after setting all properties. function list_im2_CreateFcn(hObject, eventdata, handles)

$\%$ hObject handle to list_im2 (see GCBO)

$\%$ eventdata reserved - to be defined in a future version of MATLAB

$\%$ handles empty - handles not created until after all CreateFcns called

$\%$ Hint: listbox controls usually have a white background on Windows.

$\% \quad$ See ISPC and COMPUTER.

if ispc

set (hObject, 'BackgroundColor', 'white');

else

set (hObject, 'BackgroundColor', get (0, 'defaultUicontrolBackgroundColor')) ; end

$\%$--- Executes on selection change in list_im2.

function list_im2_Callback(hObject, eventdata, handles)

$\%$ hObject handle to list_im2 (see GCBO)

$\%$ eventdata reserved - to be defined in a future version of MATLAB

$\%$ handles structure with handles and user data (see GUIDATA)

$\%$ Hints: contents $=$ get(hObject,'String') returns list_im2 contents as cell array

$\% \quad$ contents get(hObject, 'Value')\} returns selected item from list_im2

i=get (hObject, 'Value');

set (handles.list_im1, 'Value',i);

button_state = get (handles.gray_im1, 'Value');

if button_state $==$ get (handles.gray_im1, 'Max')

axes (handles.fig1);

axis image;

imshow (handles.gim1, gray);

elseif button_state $==$ get (handles.gray_im1, 'Min')

axes (handles.fig1);

axis image;

imshow (handles.im1,gray);

end

hold on;

plot (handles.pts1 (i,1) thandles.X10, handles.pts1 (i,2)+handles.Y10, ' $y+$ ') ;

hold off;

button_state $=\operatorname{get}($ handles.gray_im2, 'Value') ;

if button_state == get(handles.gray_im2, 'Max')

axes (handles.fig2);

axis image;

imshow (handles.gim2, gray);

elseif button_state $==$ get (handles.gray_im2, 'Min')

axes (handles.fig2);

axis image;

end

imshow (handles.im2, gray);

hold on;

plot (handles.pts2 (i,1)+handles.X20, handles.pts2 (i, 2)+handles.Y20, ' $y+$ ') ;

hold off;

$\%$--- Executes on button press in clear_selected.

function clear_selected_Callback(hObject, eventdata, handles)

$\%$ hObject handle to clear_selected (see GCBO) 
$\%$ eventdata reserved - to be defined in a future version of MATLAB $\%$ handles structure with handles and user data (see GUIDATA) $i=$ get (handles.list_im1, 'Value'); $\mathrm{N}=$ handles.npoints;

if $i>0$

if $i==\mathrm{N}$

if $i==1$

handles.pts1=[];

handles.pts $2=[]$;

handles . npoints $=0$;

set (handles.list_im1, 'Value',1)

set (handles.list_im2, 'Value',1);

set (handles.list_im1, 'String', [] );

set (handles.list_im2, 'String', []);

set (handles.clear_selected, 'Enable', 'off');

set (handles.clear_all, 'Enable', 'off');

set (handles.calculate, 'Enable', 'off') ;

else

handles.pts1=handles.pts1 (1:i-1,:) ;

handles.pts $2=$ handles.pts $2(1: i-1,:)$;

handles . npoints $=\mathrm{N}-1$;

set (handles.list_im1, 'Value',i-1);

set (handles.list_im2, 'Value',i-1);

s1=get (handles. list_im1, 'String');

s2=get (handles.list_im2, 'String');

set (handles.list_im1, 'String', s1(1:i-1,:));

set (handles.list_im2, 'String', s2(1:i-1,:)) ;

if $(\mathrm{N}==7)$ nd

set (handles.calculate, 'Enable', 'off');

end

elseif i>1

handles.pts1=[handles.pts1 $(1: i-1,:) ;$ handles.pts1 $(i+1: N,:)]$;

handles.pts $2=[$ handles.pts $2(1: i-1,:)$; handles.pts2 $(i+1: N,:)]$;

handles.npoints=handles . npoints -1 ;

set (handles.list_im1, 'Value', i-1)

set (handles.list_im2, 'Value', i-1);

s1=get (handles.list_im1, 'String');

s2=get (handles.list_im2, 'String');

set (handles.list_im1, 'String', [s1(1:i-1,:);s1(i+1:N,:)]);

set (handles.list_im2, 'String', [s2(1:i-1,:) ;s2 (i+1:N,: )]);

if $(\mathrm{N}==7)$ end

set (handles.calculate, 'Enable', 'off');

else

handles.pts1=handles.pts1 $(2: \mathrm{N},:)$;

handles.pts $2=$ handles.pts $2(2: \mathrm{N},:)$;

handles.npoints=handles . npoints -1

set (handles.list_im1, 'Value',1);

set (handles.list_im2, 'Value',1);

s1=get (handles.list_im1, 'String');

s2=get (handles.list_im2, 'String');

set (handles.list_im1, 'String', s1(2:N,:)) ;

set (handles.list_im2, 'String', s2(2:N,:)) ;

if $(\mathrm{N}==7)$

set (handles.calculate, 'Enable', 'off');

end

end

end

button_state $=\operatorname{get}($ handles.gray_im1, 'Value');

if button_state $==$ get (handles.gray_im1, 'Max')

axes (handles.fig1);

axis image;

imshow (handles.gim1, gray);

elseif button_state $==$ get (handles.gray_im1, 'Min')

axes (handles.fig1);

axis image;

end

imshow (handles.im1, gray);

i=get (handles.list_im1, 'Value')

hold on;

plot (handles.pts1 (i, 1) thandles.X10, handles.pts1 (i, 2)+handles.Y10, 'y+');

hold off;

button_state $=$ get (handles.gray_im2, 'Value');

if button_state $==$ get (handles.gray_im2, 'Max')

axes (handles.fig2);

axis image; 
imshow (handles.gim2, gray);

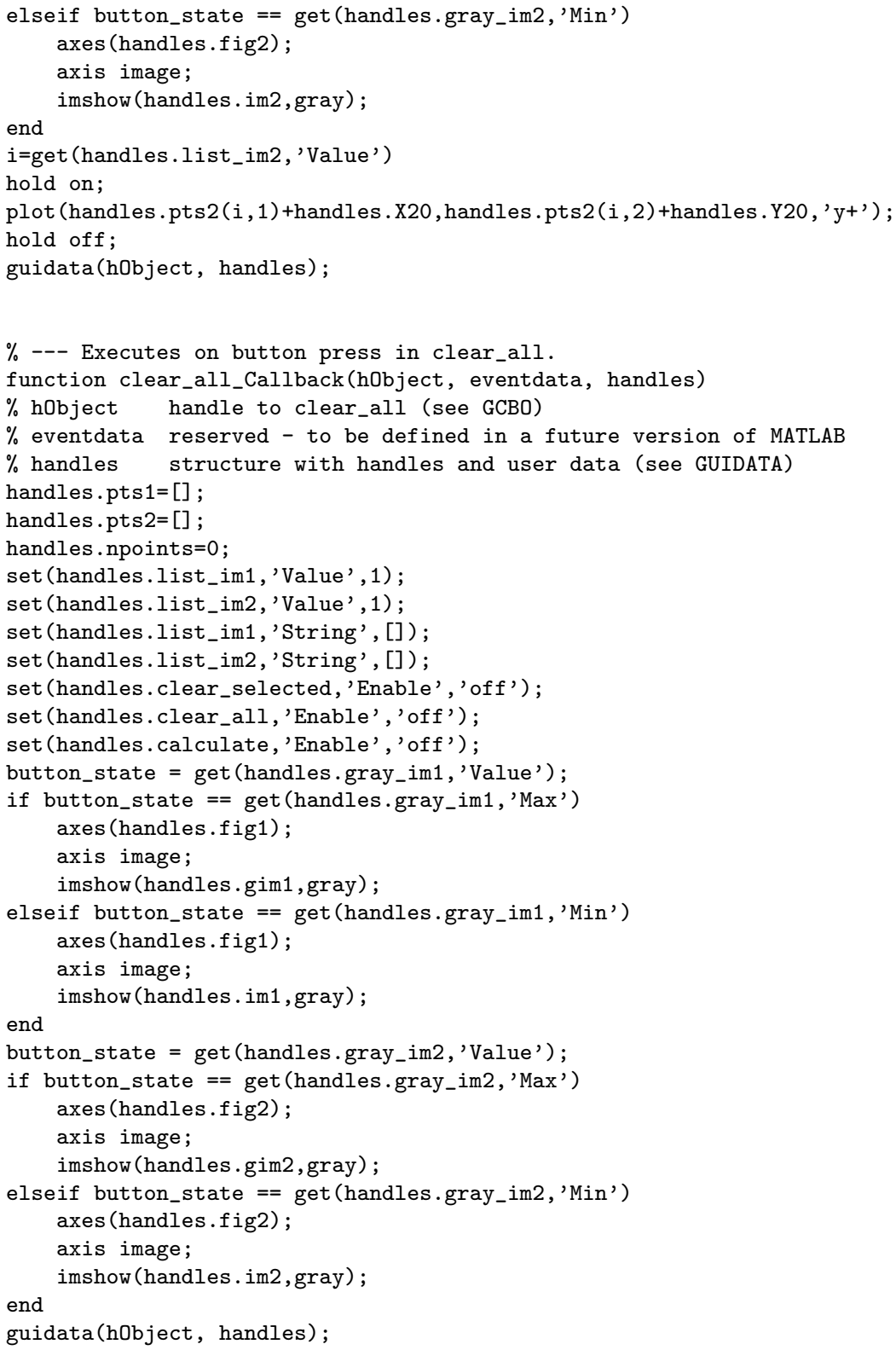


set (hObject, 'Enable' , 'off');

guidata(hObject, handles);

$\%$--- Executes on button press in save.

function save_Callback(hObject, eventdata, handles)

$\%$ hObject handle to save (see GCBO)

$\%$ eventdata reserved - to be defined in a future version of MATLAB

$\%$ handles structure with handles and user data (see GUIDATA)

im1=handles . im1;

im2=handles . im2;

$\mathrm{m} 1=$ handles $\cdot$ pts 1

$\mathrm{m} 2=$ handles. pts 2 ;

$\mathrm{N}=$ handles.npoints;

if $(\mathrm{N}>7)$

$\mathrm{F}=$ handles. $\mathrm{F}$;

e=handles .e;

[filename, pathname] = uiputfile('*.mat', 'Save Results as');

if isstr(filename) \&\& isstr (pathname)

if isempty(strfind(filename,'.mat'))

end

filename $=[$ filename '.mat' $]$;

save([pathname filename], 'im1', 'im2', 'N', 'm1', 'm2', 'F', 'e');

msgbox(['Data saved as ' pathname filename], 'Save Results', 'help', 'modal');

else

save correspondence im1 im2 $\mathrm{N}$ m1 m2 $\mathrm{F}$ e;

msgbox('Data saved in current directory as correspondence.mat', 'Save Results', ... 'help', 'modal');

end

elseif $(\mathrm{N}==7)$

$\mathrm{F} 1=$ handles. F1;

$\mathrm{F} 2$ = handles.F2

$\mathrm{a}=$ handles.a;

[filename, pathname] = uiputfile('*.mat', 'Save Results as');

if isstr(filename) \&\& isstr(pathname)

if isempty(strfind(filename,'.mat'))

end

filename $=[$ filename '.mat' $]$;

save([pathname filename], 'im1', 'im2', 'N', 'm1', 'm2', 'F1', 'F2', 'a'); else msgbox(['Data saved as' pathname filename],'Save Results', 'help','modal');

save correspondence im1 im2 $\mathrm{N} \mathrm{m} 1 \mathrm{~m} 2 \mathrm{~F} 1 \mathrm{~F} 2$ a;

msgbox('Data saved in current directory as correspondence.mat', 'Save Results', ... 'help', 'modal');

end

end

set (handles.calculate, 'Enable', 'on');

set (handles.clear_selected, 'Enable', 'on') ;

set (handles.clear_all, 'Enable', 'on');

set (handles.collect_im1, 'Enable', 'on') ;

set (handles.collect_im2, 'Enable', 'on');

set (hObject, 'Enable', 'off');

$\%$--- Executes on button press in collect_im1.

function collect_im1_Callback(hObject, eventdata, handles)

$\%$ hObject handle to collect_im1 (see GCBO)

$\%$ eventdata reserved - to be defined in a future version of MATLAB

$\%$ handles structure with handles and user data (see GUIDATA)

\% Hint: get(hObject,'Value') returns toggle state of collect_im1

set (handles.collect_im1, 'Enable', 'off');

set (handles.collect_im2, 'Enable', 'off');

button $=1$;

while button $==1$

axes (handles.fig1);

[x1, $\mathrm{y} 1$, button] =ginput (1);

if button $==1$

hold;

plot (round (x1), round (y1), ' $\mathrm{y}^{+}$');

hold;

s1=get (handles. list_im1, 'String')

s1= [s1; sprintf ('(\%04d, \%04d)', round (x1), round (y1))];

set (handles.list_im1, 'String', s1);

axes (handles.fig2)

$[\mathrm{x} 2, \mathrm{y} 2]=\operatorname{ginput}(1)$;

hold on; 


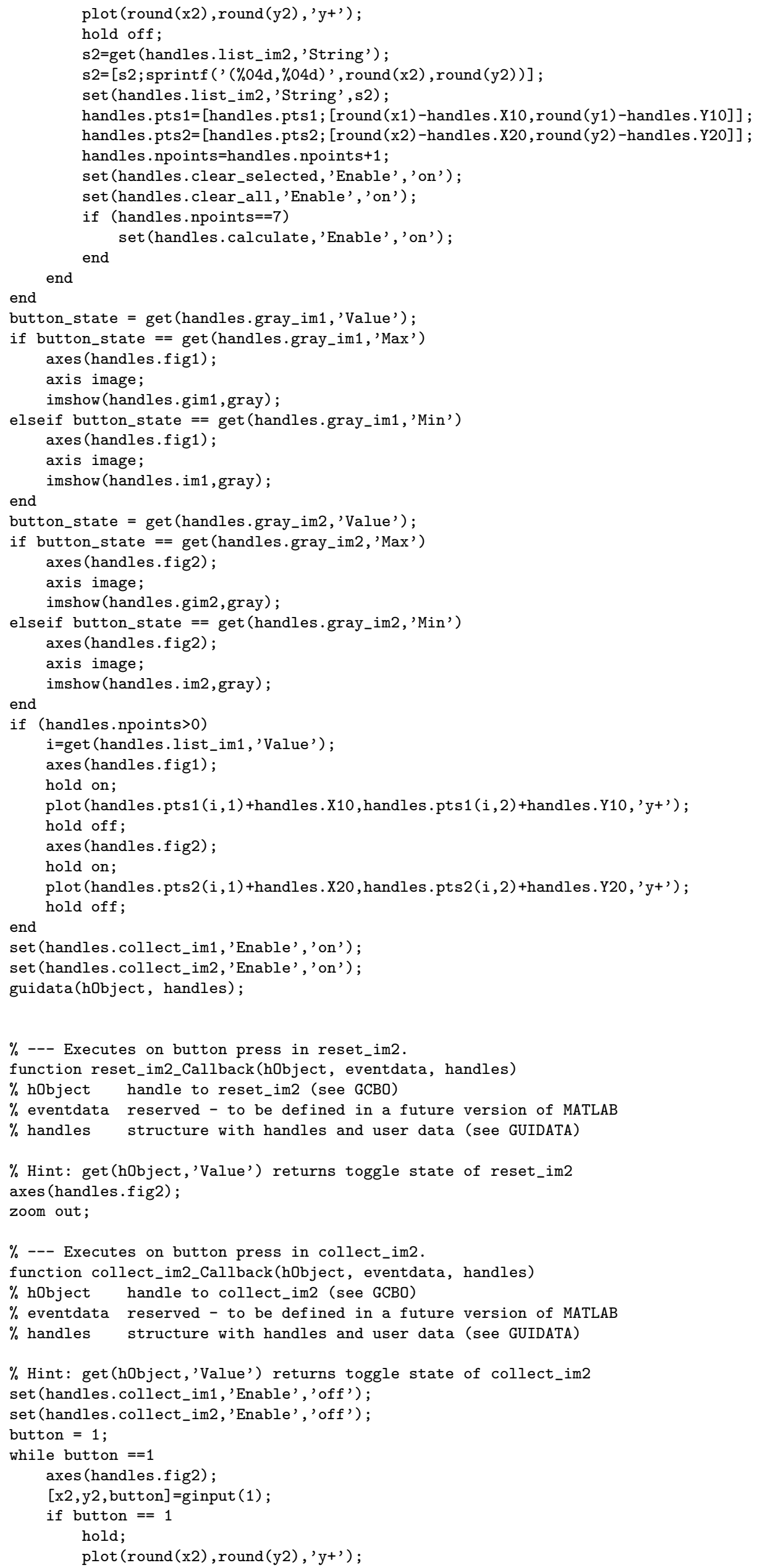




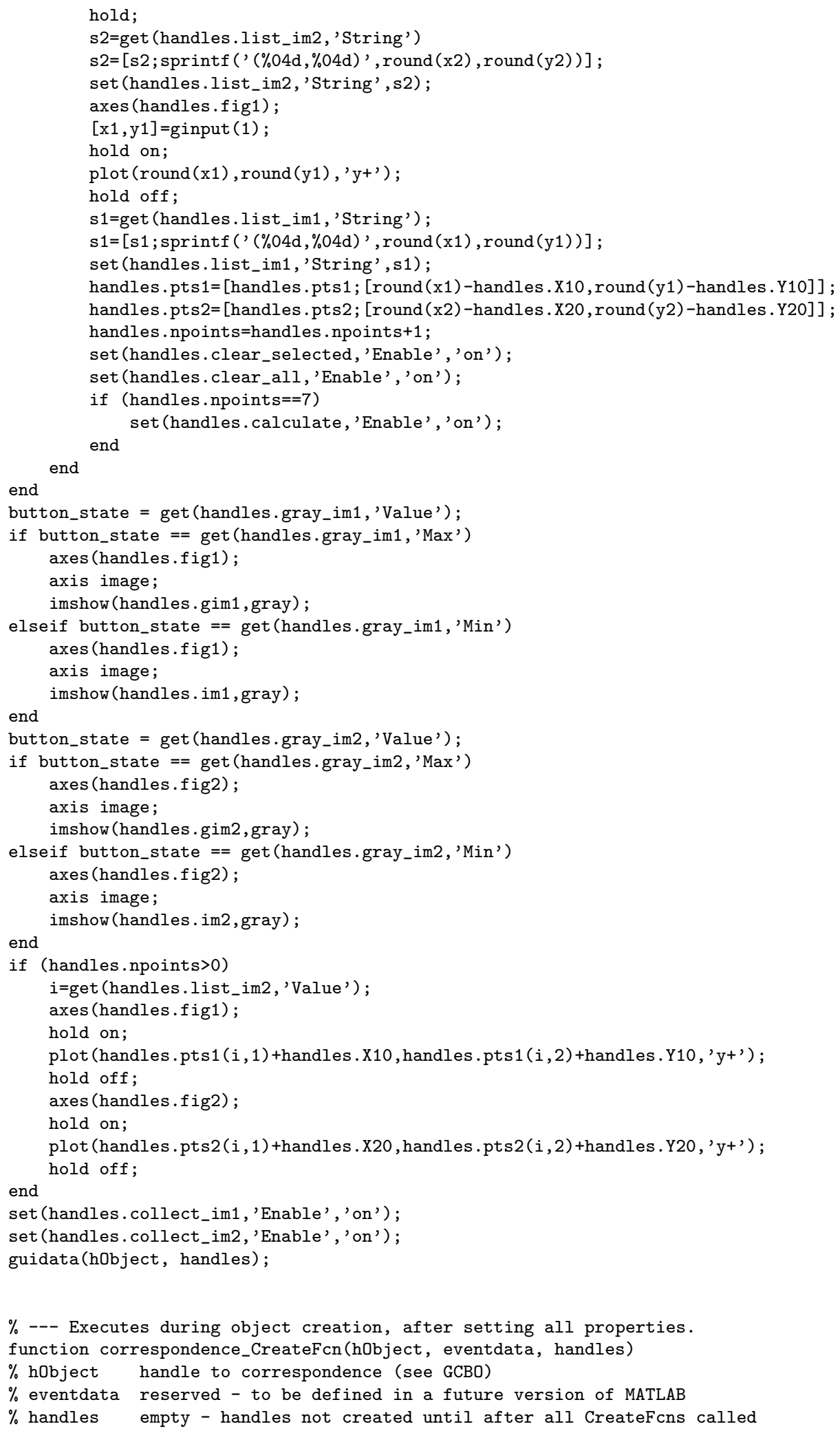

$\%$--- Executes during object creation, after setting all properties.

function correspondence_CreateFcn(hObject, eventdata, handles)

$\%$ hObject handle to correspondence (see GCBO)

$\%$ eventdata reserved - to be defined in a future version of MATLAB

$\%$ handles empty - handles not created until after all CreateFcns called

\section{B.7 Outros Programas Acessórios}

\section{B.7.1 Matriz do Produto Vetorial}




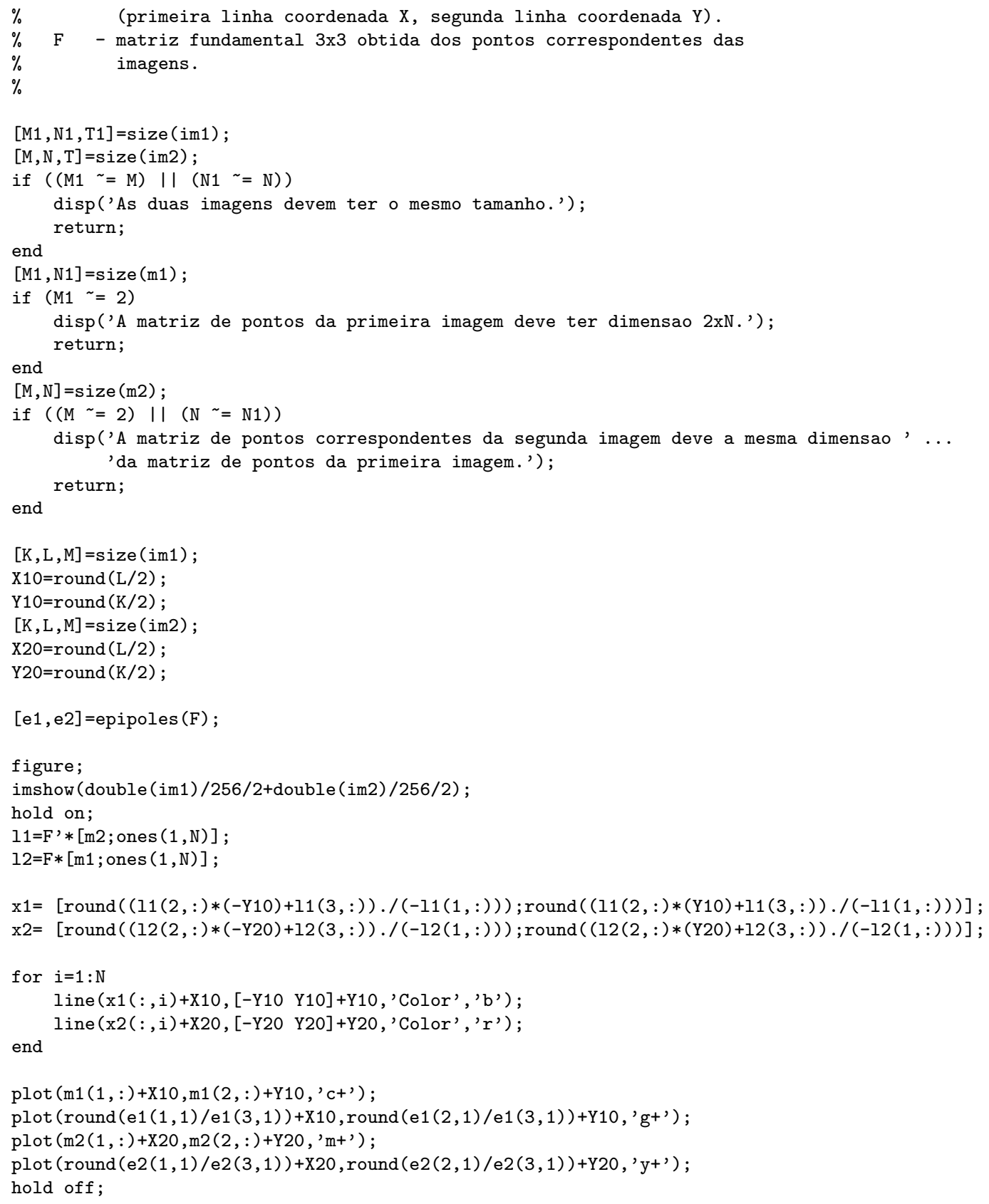

